\title{
PATTERNS OF TEMPORAL AND SPATIAL
}

\author{
VARIABILITY OF
}

SPONGE ASSEMBLAGES

Jade Berman

\begin{abstract}
A thesis submitted to
Victoria University of Wellington

in fulfillment of the requirements for the degree of

Doctor of Philosophy in Science
\end{abstract}

2012

\section{VICTORIA UNIVERSITY OF WELLINGTON}

Te Whare Wānanga o te Ūpoko o te Ika a Māui

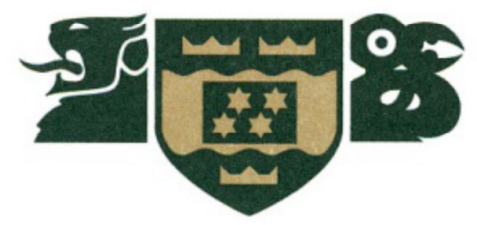


ABSTRACT 
The primary goals of this thesis were to understand the spatial and temporal pattern of sponge assemblage variation over a variety of scales and investigate suitable monitoring methods for sponge assemblages. Sponges are an ecologically significant group in benthic marine communities, which are often ignored in current monitoring schemes. In chapter two the sponge biodiversity of New Zealand waters to $200 \mathrm{~m}$ was examined using Taxonomic Distinctness measures initially to test if genera data could be used as a proxy for species level data in New Zealand waters. It was found that over $50 \%$ of the variation in genera biodiversity could be explained by location and depth around New Zealand. The study helped pinpoint where there were gaps in the New Zealand dataset, in particular for the West Coast of the South Island and also areas such as the Wellington South Coast, which had higher than expected values for Average and Variation Taxonomic distinctness measures, which as important areas where sponges should be monitored to make sure the high levels of biodiversity are protected. Taxonomic distinctness measures are useful for initially assessing how the biodiversity is distributed, especially when using a data set with uneven sampling effort, as it is robust to spatial and temporal bias in the majority of cases. However, there was an outlier to the genera data correlating well with the variation in species data in the case of a site dominated by Haliclona sp (Lyttelton Harbour). In chapters three and four the spatial and temporal variability of sponge assemblages of the Wellington South Coast were explored creating both a species list for the area and an understanding of how the sponge assemblage varies over time and space. There were significant differences in the sponges assemblages in similar habitat types over a scale of a few hundred metres. In addition, although all the sponge assemblages changed seasonally, the changes at each sampling site responded in a slightly different way most likely due to spatiotemporal variation in environmental conditions. A similar seasonal pattern was also observed in chapter five for sponge assemblages at Skomer Marine Reserve and this pattern was also clear when using morphological monitoring methods. This means that once a site has been mapped for biodiversity it is possible for some habitats to use morphological monitoring to identify if the sponge assemblage is changing 
significantly saving time and money. The results from Indonesia (chapter six) showed that although the sponge assemblages were changing significantly in the actual species present and their abundances, the proportion of diversity within each spatial level (quadrat, site and region) remained consistent when sampled at the same time each year throughout the five year study. In species rich assemblages there are a variety of life strategies that can respond differently to shifts in environmental conditions and contribute to ecological functioning in various ways. Various monitoring methods have been tested using sponge assemblages over various spatial and temporal scales in this thesis. Spatial, temporal and the interaction of spatial and temporal factors were all important for identifying significant assemblage differences at all of the sites. Further studies integrating the interaction of spatial and temporal factors into understanding monitoring data sets are vital to understand the patterns of assemblage variability and therefore incorporate into habitat management plans. 
DECIPHERING SPONGES THROUGH TIME AND SPACE...

Sponges are not as you may suppose,

A funny kind of plant.

They are in fact cool animals,

Like bats kiwis or ants.

So why do I

into the deep sea plunge,

Taking on my PhD

studying the almighty sponge?

Well you find them all over the world from Taupo hot pools,

To Antarctic quarters,

All over the tropics

and Wellingtons waters.

(which are not tropical I'd like to add...)

They have many functional roles

Such as filtering the sea,

Gluing coral reefs together

And making medicines for you and me.

Yet sponges are often ignored

or described as one entity

In monitoring schemes

designed to keep our seas healthily

So my task is to understand sponge assemblage variability

What are the normal patterns

and what's their unpredictability

So far I have found out,

The world all around us is vast and complex

There are seasonal patterns

but the scales often perplex

There are some patterns

over different temporal scales

But micro environmental conditions

cause spatial factors to prevail.

Significant differences can be seen over the scale of a few metres

Even when at first glance they look like good repeaters

And replicate sites with the same macro conditions 
Can vary considerably in species compositions

The scale of natural variability is important to note And the residual flexibility of the assemblage to promote As the sponges often undergo large annual fluctuations Which then return eventually to similar combinations

It is possible even for non scientific divers

To see significant differences

Through morphological change drivers

So sponges can be incorporated into monitoring schemes

Which through morphological methods

Can spot trouble before they disappear into dreams

In fact their resilience may lead to a good job for me As sponges outlive climate extremes and take over the sea.

J. Berman (submitted for the 2010 "Three minute thesis competition") 


\section{ACKNOWLEDGEMENTS}

Over the time I have been in New Zealand I have met, worked with, and become friends with a wide range of people. Although this thesis has my name on the cover, I would not have been able to write these final words without the emotional and physical support that came in many forms from numerous people and the organizations they work for.

Firstly I want to thank my family, and in particular my parents Gi and Barbara Berman for letting me be a boomerang kid and come back for three months intensive writing at the family home, taking over the computer and providing me with emotional and edible support.

I would also like to thank my supervisor James Bell who has always been amazingly quick to review my work and patiently listened to my many ideas and try and keep me steered along the steady track towards completion of this thesis. He is an inspiration with his ability to manage so many students and yet still publish many papers of his own at the same time.

I would not have been able to start my PhD let alone finish without funding, therefore I am grateful for: my New Zealand Educated International Doctoral Scholarship, Victoria University Wellington Science Faculty Research grants for attending the International Sponge Conference 2010 and INTERCOL 2010 plus funds to work at the Queensland Museum, in Brisbane. Victoria University also provided me with a submission scholarship and the Postgraduate Students Association also provided me with a grant towards attending the International Sponge Conference. British Ecological Society also provided me funding towards both my International conferences. New Zealand Marine Sciences Society provided me with awards at their conferences for the best Poster (2008) and the best oral presentation on Conservation in New Zealand (2010). Department of Conservation (Helen Kettles) provided the permit to work in Taputeranga Marine Reserve (docDM-336985) and also Callum and team at 
Paraninihi and the White Island DOC Survey team for allowing me to join you.

I could not have written my first data chapter on New Zealand sponge patterns without the help of Michelle Kelly who kindly allowed me access to the NIWA sponge database and checked my identifications, Franzino Smith who helped me develop my ideas for this huge chapter, and let me have access to his pictures and database, as well as taking me along to White Island to carry out surveys to add to national sponge knowledge. The PRIMER and PERMANOVA course with Bob Clarke and Marty Anderson provided me with the inspiration to write this chapter and also made all the other chapters within this thesis possible. Tim Jones and Phil Neubauer both helped me stay on the statistical straight and narrow in order to interpret and present my data in the most suitable manner.

Many people have helped me in the field including for Wellington: Alejandra Perea-Blázquez, Alejandro Perez Matus, Anna Smith, Mat Kertez, Nick Ward, Pelayo Salinas de Leon, Phil Neubauer, Steve Lindfield, Tyler Eddy, William Arlidge, Tamsen Byfield, Bioblitz Divers, Phil Neubauer. For Skomer; through the Countryside Council for Wales; Mark Burton, Philip Newman, Rob Gibbs, Kate Lock, F. Bunker and J. Jones. For Indonesia; Lucy Sprung, Sonia Rowley, Abi Powell, Heather Murray, Steve Lindfield, Pelayo Salinas de Leon and Operation Wallacea for letting me work on site. Once I brought back samples to the lab I had lots of help to process them from Alejandra Perea-Blázquez, Ingrid Knapp, Abi Powell, and Susanna Govella. Once I had the samples processed and I had identified them, I then received lots of help from John Hooper and Trish Sutcliffe at the Queensland Museum to match my samples from Indonesia with their huge samples database where possible.

Many thanks to the all staff at the SBS, including 'Snout' who made diving possible, John who kept the lab ticking over, Patricia who helps keep me an active student, Sandra and Mary without whom SBS would not function and Paul who gives me money whenever I approach with timesheets or grant requests. 
Thanks to Claire Pascoe and Zoe Studd for helping me to balance running the Experiencing Marine Reserves Programme around my PhD and I certainly could not have run the programme at all without the help of many people from the lab, housemates and friends who didn't know quite what to expect. I could not have got through this thesis without the support of my extended New Zealand family. Early people such as Pelayo, Monica, Duncan, Gesine, Laura, Michelle, Barnaby and the Chilean posse provided me with instant support when I first landed in Wellington and an instant set of friends. The posse has evolved and people have left and Chileans have tag teamed in and out but I have many fond memories from my first days in 411. Members of MDG for providing necessary support in my final months including Cesar, Shane, Erasmo, Danelle, Phil, Emilie, Tim, Abi, Ingrid, Ursula, Danni, Tall Paul, Laura, Jamie, Jeanine, Sergio, Anita, Nicole, Jeff, Sonja, Rachel, Fernanda, James, Sandra, Bionda, Gareth, Bex, Tyler, Ale, Alejandra and Benja.

Special thanks go to my friends who have coped with me in particular over the last few months of craziness and kept me sane outside the lab, Anna, Ewa, Lorraine, Amane, Steve, Donald, Brock, Caroline, Liz, Jof, Paul, Ethel, Xavier, Gavin, Halley, Hayden, Isabella, Jeremie, Katie, Matilide, Laura, Liviu, Richard, Ros, Sarah, Sonja, Maya, Hillary, Emmy, Zoe, Claire, Niamh, Jayne, Vidette and Chanelle. You have been my rocks to keep me in the hard place until I finish and you are all important to me in so many ways. Thank you. 


\section{CONTRIBUTIONS AND PUBLICATIONS}

This thesis has been adapted from a series of manuscripts which are in the review process or to be submitted in the near future. All fieldwork, lab-work and writing were conducted by the author, apart from the following exceptions: Chapter 2

Study Design: Author

Data Collection: Main database was adapted from the NIWA Porifera database compiled by M. Kelly through the samples collected and identified as part of ongoing NIWA research voyages studying the ecology of seamounts and other coastal expeditions funded by FRST Contracts C01Z0028 (Seamounts) C01X0219 (Biodiversity and Biosecurity). Additional data collection trips were made by the Author, J. Bell and F. Smith.

Analysis: Author carried out all the analyses except for the creation of GAM models in $\mathrm{R}$ where $\mathrm{T}$. Jones assisted the author.

Writing and Editing: The author with editing by J. Bell.

Publication: In progress in conjunction with J. Bell and M. Kelly.

\section{Chapter 3}

Study Design: Author and J. Bell

Data Collection: Author

Analysis: Author and M. Kelly from NIWA confirmed the taxonomic identifications made by author.

Writing and Editing: Author with Editing from J. Bell.

Publications: Berman, J. \& Bell, J.J. (2010) Spatial variability of sponge assemblages on the Wellington South Coast, New Zealand. The Open Marine Biology Journal, 4, 12-25

Berman, J., Perea-Blázquez, A., Kelly, M., \& Bell, J. J. 2008. Sponges of the Wellington South Coast. In Gardner, JPA (editor). 2008. The Taputeranga Marine Reserve. First Edition Publishers, Wellington, New Zealand

Chapter 4

Study Design: Author and J. Bell

Data Collection: Author 
Analysis: Author

Writing and Editing: Author with Editing from J. Bell.

Publication: In progress in conjunction with J. Bell

\section{Chapter 5}

Study Design: Author and J. Bell

Data Collection: Author, J. Bell, and the Countryside Council for Wales team (M.

Burton, K. Lock, R. Gibbs and P. Newman)

Analysis: Author

Writing and Editing: Author with Editing from J. Bell.

Publication: In progress in conjunction with J. Bell and J. Burton. Initial results were published in the report below.

Berman, J., Burton, M. and Bell, J.J. 2009 Sponge assemblages report - CMS code: RM13/01 In: Skomer Marine Nature Reserve Project Report 2008/9. Countryside Council for Wales.

\section{Chapter 6}

Study Design: Author and J. Bell

Data Collection: Author and J. Bell,

Analysis: Author, with J. Hooper and P. Sutcliffe at the Queensland Museum confirming the author's taxonomic identifications.

Writing and Editing: Author with editing from J. Bell.

Publication: In progress in conjunction with J. Bell.

The author also contributed to the paper below through the taxonomic analysis of the samples as part of the work in this chapter.

Bell, J., Berman, J., Jones, T. \& Hepburn, L. (2010) Variability in the spatial association patterns of sponge assemblages in response to environmental heterogeneity. Marine Biology, 157, 2503-250 
Chapter 1 General Introduction 1

Chapter 2 Spatial patterns of sponge biodiversity in New Zealand's 11 coastal waters

$\begin{array}{lll}2.1 & \text { Abstract } & 12\end{array}$

$\begin{array}{lll}2.2 & \text { Introduction } & 13\end{array}$

$\begin{array}{lll}2.3 & \text { Methods } & 22\end{array}$

$\begin{array}{lll}2.3 .1 & \text { Data sources } & 22\end{array}$

$\begin{array}{lll}2.3 .2 & \text { Study area } & 23\end{array}$

$\begin{array}{lll}\text { 2.3.3 Species and genera level analysis } & 23\end{array}$

2.3.4 Sponge biodiversity patterns around the New Zealand EEZ 27

$\begin{array}{ll}\text { 2.3.5 Sponge biodiversity within bioregions } & 32\end{array}$

$2.4 \quad$ Results 33

2.4.1 Species and genera level analysis 33

2.4.2 Biodiversity in the EEZ 36

$\begin{array}{lll}2.4 .3 & \text { Sponge Bioregions } & 45\end{array}$

$\begin{array}{lll}2.5 & \text { Discussion } & 48\end{array}$

2.6 Appendix of the model fitting for sponge biodiversity 60 around New Zealand for AvTD and VarTD values

Chapter 3 Spatial variability of sponge assemblages on the Wellington 69 South Coast, New Zealand.

$\begin{array}{lll}3.1 & \text { Abstract } & 70\end{array}$

$\begin{array}{lll}3.2 & 71\end{array}$

$\begin{array}{lll}3.3 & \text { Methods } & 73\end{array}$

$\begin{array}{lll}\text { 3.3.1 Study area } & 73\end{array}$

$\begin{array}{lll}3.3 .2 & \text { Biodiversity survey } & 74\end{array}$

$\begin{array}{lll}3.3 .3 & \text { Spatial survey } & 76\end{array}$

$\begin{array}{lll}3.4 & \text { Results } & 78\end{array}$

$\begin{array}{lll}3.4 .1 & \text { Biodiversity Survey } & 78\end{array}$

$\begin{array}{lll}3.4 .2 & \text { Spatial Survey } & 82\end{array}$

$\begin{array}{lll}3.5 & \text { Discussion } & 90\end{array}$

Chapter 4 Temporal variability of sponge assemblages on the 97

$\begin{array}{lll}4.1 & \text { Abstract } & 98\end{array}$

$\begin{array}{llr}4.2 & 99\end{array}$

$\begin{array}{lll}4.3 & 103\end{array}$

$\begin{array}{lll}4.3 .1 & \text { Study area } & 103\end{array}$

$\begin{array}{lll}\text { 4.3.2 Study methodology } & 104\end{array}$

$\begin{array}{ll}\text { 4.3.3 Environmental data } & 106\end{array}$

$\begin{array}{lll}4.4 & \text { Results } & 108\end{array}$

$\begin{array}{lll}\text { 4.4.1 Temporal variability of the assemblages } & 108\end{array}$

$\begin{array}{ll}\text { 4.4.2 Temporal variability of the important species } & 113\end{array}$

$\begin{array}{ll}4.4 .3 & 120\end{array}$

$\begin{array}{lll}4.5 & 125\end{array}$

$\begin{array}{lll}\text { 4.5.1 Temporal variability of the assemblages } & 125\end{array}$

$\begin{array}{lll}\text { 4.5.2 Temporal variability of the important species } & 129\end{array}$ 
$\begin{array}{lll}\text { 4.5.3 Environmental data } & 131\end{array}$

4.6 Appendix SIMPER Similarity Percentages - Two-way 133

analysis of species contributions of site crossed with season

Chapter 5 Spatial and temporal variability of sponge species and 141

morphological diversity in a temperate marine reserve

$\begin{array}{lll}5.1 & \text { Abstract } & 142\end{array}$

$\begin{array}{lll}5.2 & \text { Introduction } & 143\end{array}$

$\begin{array}{lll}5.3 & 146\end{array}$

$\begin{array}{lll}\text { 5.3.1 Study Area } & 146\end{array}$

$\begin{array}{lll}\text { 5.3.2 Data Analysis } & 148\end{array}$

$\begin{array}{lll}\text { 5.3.3 Statistical Analysis } & 149\end{array}$

$\begin{array}{lll}\text { 5.3.4 } & 152\end{array}$

$\begin{array}{llr}5.4 & \text { Results } & 153\end{array}$

$\begin{array}{lll}\text { 5.4.1 } & \text { Species assemblage data } & 153\end{array}$

$\begin{array}{ll}\text { 5.4.2 } & 162\end{array}$

5.4.3 Correlations between the species and morphological data 164

$\begin{array}{lll}\text { 5.4.4 Environmental data correlations } & 166\end{array}$

$\begin{array}{lll}5.5 & \text { Discussion } & 171\end{array}$

5.5.1 Patterns of seasonal variability in the sponge species 171

5.5.2 Patterns of seasonal variability in the sponge morphological 173 assemblages in Skomer MNR

5.5.3 The correlations of species and morphological assemblage $\quad 175$

variability

5.5.4 The correlations of environmental factors with Skomer 176

MNR sponge assemblage variability

$\begin{array}{lll}\text { 5.5.5 Monitoring Recommendations } & 177\end{array}$

5.6 Appendix SIMPER Similarity Percentages - Two-way 179

analysis of species contributions of site crossed with season

$\begin{array}{lll}\text { Chapter } 6 & \text { Temporal and spatial diversity patterns of sponge } & 187\end{array}$

assemblages in the Wakatobi Marine National Park, Indonesia.

$\begin{array}{llr}6.1 & \text { Abstract } & 188\end{array}$

$\begin{array}{lll}6.2 & 189\end{array}$

$\begin{array}{lll}6.3 & \text { Methods } & 194\end{array}$

$\begin{array}{lll}\text { 6.3.1 Study Area } & 194\end{array}$

$\begin{array}{ll}\text { 6.3.2 Data collection } & 195\end{array}$

$\begin{array}{lll}\text { 6.3.3 Statistical Analysis } & 196\end{array}$

$\begin{array}{lll}6.4 & \text { Results } & 199\end{array}$

6.5 Discussion 208

6.6 APPENDIX - SIMPER Similarity Percentages - Two-way 213

analysis of species contributions of site crossed with

season.

$\begin{array}{lll}\text { Chapter } 7 & \text { General Discussion } & 225\end{array}$

References 235 
Fig. 2.1 Major coastal current systems of New Zealand 16

$\begin{array}{lll}\text { Fig. 2.2 Current New Zealand biogeoclassification schemes. } & 17\end{array}$

Fig. 2.3 A typical sponge garden assemblage at 18m depth at 19 Paraninihi MPA, Taranaki.

Fig. 2.4 Porifera records around mainland New Zealand. 30

Fig. 2.5 The relationship between genera and species diversity. 33

Fig. 2.6 The relationship between AvTD for genera and species port 34

Fig. 2.7 The relationship between VarTD and AvTD for genera and 35 species data.

Fig. 2.8 MDS plot showing sponge assemblage changes with depth 38

Fig. 2.9 AvTD and VarTD variability with depth changes in New 39 Zealand waters.

Fig. 2.10 AvTD and VarTD variability with latitude changes in New 40 Zealand waters

Fig. 2.11 AvTD and VarTD variability with longitude changes in New 41 Zealand waters.

Fig. 2.12 AVTD and VarTD models related to the New Zealand 44 coastline.

Fig. 2.13 MDS plot of DoC bioregions (0-50m). 46

Fig. 2.14 MDS plot of Shears (2008) bioregions (0-50m). 46

Fig. 2.15 Spatial distribution sponges in New Zealand using a mean 52 rank of six biodiversity measures.

$\begin{array}{lll}\text { Fig. 3.1 Surveyed sites along the Wellington South Coast, New } & 73\end{array}$ Zealand.

Fig. 3.2 Spicules and spongin fibres from sponges of the Wellington 75 South Coast.

Fig. 3.3 Common sponge species from the Wellington South Coast. $\quad 79$

Fig. 3.4 Species accumulation curves for the Wellington South Coast. $\quad 82$

Fig. 3.5 Species accumulation curve comparing Breaker Bay, the Far 83

Fig. 3.6 Sponge density $\left(\mathrm{m}^{-2}\right)$ at Breaker Bay, the Far Sirens and the 84 Near Sirens sites for the fifteen most abundant sponge species.

Fig. 3.7 Percentage cover of the fifteen most abundant sponge 84 species at Breaker Bay, Far Sirens and Near Sirens sites.

Fig. 3.8 Non-metric multidimensional scaling ordinations (nMDS plots in PRIMER) of the abundance within Wellington South Coast sponge assemblages.

Fig. 3.9 Non-metric multidimensional scaling ordinations (nMDS 86 plots in PRIMER) of the percentage cover within the Wellington South Coast sponge assemblages.

Fig. 4.1 Sponge species richness ( \pm S.E.) for the Wellington South 108 Coast.

Fig. 4.2 Sponge density ( \pm S.E.) for the Wellington South Coast 
Fig. 4.3 Non-metric multidimensional scaling ordinations of the seasonal changes in sponge density of three sites along the Wellington South Coast.

Fig. 4.4 The seasonal densities and S.E. bars for Clathrina sp.2 116

$\begin{array}{lll}\text { Fig. 4.5 The seasonal densities and S.E. bars for Clathrina sp.3 } 116 & 116\end{array}$

$\begin{array}{lll}\text { Fig. 4.6 The seasonal densities and S.E. bars for Leucosolenia echinata } & 117\end{array}$

$\begin{array}{lll}\text { Fig. 4.7 The seasonal densities and S.E. bars for Sycon sp. } & 117\end{array}$

Fig. 4.8 The seasonal densities and S.E. bars for Oscarella lobularis 118

Fig. 4.9 The seasonal densities and S.E. bars for Halisarca sp. 118

Fig. 4.10 The seasonal densities and S.E. bars for Plakina c.f. trilopha 119

Fig. 4.11 The seasonal densities and S.E. bars for Stelletta sp. 119

Fig. 4.12 12 The mean seawater temperature in oC \pm S.E. for the Near 120 Sirens and Far Sirens sites from July 2008 - May 2009 at 9am.

Fig. 4.13 Monthly mean air temperatures for the Wellington South 122 Coast.

Fig. 4.14 Monthly highest wind gust speeds for the Wellington South 123 Coast.

Fig. 4.15 Total monthly rainfall for the Wellington South Coast. 124

Fig. 4.16 Mean monthly air pressure for the Wellington South Coast 124

$\begin{array}{lll}\text { Fig. 5.1 Map of study sites within Skomer MNR. } & 147\end{array}$

Fig. 5.2 Morphological groups used to classify the sponges of Skomer 149 MNR.

Fig. 5.3 Some of the common species and morphologies found within 153 the Skomer MNR

Fig. 5.4 Mean sponge species/entities per $\mathrm{m}-2 \pm \mathrm{SE}$ at the three Skomer sites (SH, BG, and DL) in spring, summer and autumn 2006-2009.

Fig. 5.5 Mean No. of sponges per m-2 \pm S.E at the three sites (SH, BG, 157 and DL) in spring, summer and autumn 2006-2009.

Fig. 5.6 Multi-dimensional scaling (MDS) plot of sponge assemblages 158 based on the species data across the three sites (SH, BG \& DL) at Skomer MNR showing the spatial, seasonal and annual variability.

Fig. 5.7 Seasonal mean abundances $( \pm$ S.E) of Hymeraphia stellifera, 161 Halicnemia patera, Haliclona (Haliclona) oculata and Haliclona (Haliclona) urceolus within Skomer MNR.

Fig. 5.8 Multi-dimensional scaling (MDS) plot of sponge assemblages 162 based on the morphological data at the three sites (SH, BG, and DL).

Fig. 5.9 MDS plots of the seasonal shifts in species and morphological 165 assemblages in Skomer MNR.

Fig. 5.10 Variation in Seawater temperature from 2006-2009. 167

Fig. 5.11 Variation in air temperature (mean, maximum and 168 minimum) from 1993-2009.

Fig. 5.12 Variation in net radiation per month from 2007-2009. 168

Fig. 5.13 Monthly maximum wind gust speed m/sec from 2007-2009. 169

Fig. 5.14 Variation in water visibility using the sechi disc from 2006- 170 2009. 
Fig. 6.1 Location of survey sites off Hoga Island, South-East Sulawesi 194

Fig. 6.2 Some of the common sponge species found within the Hoga 199 sites.

Fig. 6.3 Mean sponge species/entities per $\mathrm{m}-2 \pm \mathrm{SE}$ at the three Hoga 201 sites (A, B, and C) in August 2005-2009.

Fig. 6.4 Mean No. of sponges per $\mathrm{m}^{-2} \pm$ S.E at the three Hoga sites (A, B 202 and C) from 2005-2009.

Fig. 6.5 Interpolated SOBS and Chao 2 Curves 2005-2009 with S.E for 203 Hoga sponge O.T.U's and abundance of individuals across all three sites.

Fig. 6.6 Additive partitioning of the diversity into alphaquadrat 204 betasite betaregion components by species richness, Simpson Index and Shannon Index across all three sites, Hoga.

Fig. 6.7 Multi-dimensional scaling (MDS) plot of sponge assemblages 205 based on the species diversity data at the three sites A, B, \& C in August 2005-2009.

Fig. 6.8 Annual mean abundances in August 2005-2009 ( \pm S.E) of 207 Protosuberites sp. (1), Sycon sp. (73), Pericharax sp. (10) and Ecionemia c.f. acervus (8) within Hoga.

Fig. 6.9 Mean percentage cover $( \pm$ S.E) of macrobenthic assemblage $\quad 210$ types identified form photographs at the three study sites, Hoga, Indonesia in August 2006 and 2007

Fig. 7.1 A flowchart to assist in identifying sampling areas that are 228 sponge biodiversity hotspots and therefore need to be incorporated into habitat management and monitoring schemes. 
Table 2.1 Branch weighting values for calculating taxonomic 26 distinctness indices.

Table 2.2 The twenty commonest genera recorded from 0- 37 200m within the New Zealand EEZ.

Table 2.3 Values for AvTD and VarTD for sponge assemblages with more than ten genera present in New Zealand waters

Table 2.4 AvTD models for $150 \mathrm{~m}$ or shallower sorted in ascending order of AIC (lowest AIC is the best model)

Table 2.5 AvTD models for $50 \mathrm{~m}$ or shallower (lowest AIC is the best model)

Table 2.6 VarTD models for $150 \mathrm{~m}$ or shallower sorted in ascending order of AIC (lowest AIC is the best model)

Table 2.7 VarTD models for $50 \mathrm{~m}$ or shallower sorted in ascending order of AIC (lowest AIC is the best model)

Table 2.8 No. of genera and AvTD and VarTD values for the DOC

Table 2.9 No. of genera and AvTD and VarTD values for the

Table 3.1 Sponge taxa listed in alphabetical order recorded

Table 3.2 SIMPER analysis of the percentage similarity within and between sites in relation to sponge abundance (log transformed).

Table 3.3 SIMPER analysis of the percentage similarity within and between sites in relation to sponge abundance (Dispersion Index down-weighted).

Table 3.4 SIMPER analysis of the percentage similarity within and between sites in relation to sponge percentage cover.

Table 3.5. Global values for sponge species richness.

Table 4.1 Design for the PERMANOVA model for the analysis of the multivariate species and morphological data in response to the factors listed.

Table 4.2 PERMANOVA results of the species assemblage data.

Table 4.4 Species explaining the similarity across all sites in

Table 4.5 Species explaining the similarity across all sites in

Table 4.6 Species explaining the similarity across all sites in winter

Table 4.7 Species explaining the differences between autumn and spring. 
Table 4.8 Species explaining the differences between autumn

and summer

Table 4.9 Species explaining the differences between spring

135

and summer.

Table 4.10 Species explaining the differences between autumn and winter

Table 4.11 Species explaining the differences between spring and winter

136

136

Table 4.12 Species explaining the differences between summer and winter.

Table 4.13 Species explaining the similarities within the Breaker Bay quadrats.

Table 4.14 Species explaining the similarities within the Far Sirens quadrats.

Table 4.15 Species explaining the similarities within the Near Sirens quadrats.

Table 4.16 Species explaining the differences between Breaker Bay and the Far Sirens quadrats across all seasons.

Table 4.17 Species explaining the differences between Breaker Bay and the Near Sirens quadrats across all seasons.

Table 4.18 Species explaining the differences between Far Sirens and the Near Sirens quadrats across all seasons.

Table 5.1 Permanent quadrat surveys carried out at Thorn Rock since July 2006

Table 5.2 PERMANOVA model design for the analysis of the multivariate species and morphological data in response to the factors listed

Table 5.3 Sponge species and morphologies found at the Skomer MNR.

Table 5.4 PERMANOVA results of the species assemblage data.

Table 5.5 PERMANOVA results of the morphology assemblage

Table 5.6 Spearman's rank correlations between the morphological and species data sets.

Table 5.7 Species explaining the similarities within Broad Gulley across all seasons.

Table 5.8 Species explaining the similarities within Dog Leg

Table 5.9 Species explaining the similarities within Spongy

Table 5.10 Species explaining the differences between Broad Gulley and Dogs Leg across all seasons.

Table 5.11 Species explaining the differences between Broad Gulley and Spongy Hillocks across all seasons.

Table 5.12 Species explaining the differences between Dogs Leg and Spongy Hillocks across all seasons.

Table 5.13 Species explaining the similarities within spring across all sites. 
across all sites.

Table 5.15 Species explaining the similarities within autumn 182

across all sites.

Table 5.16 Species explaining the differences between spring 182

and summer across all sites.

Table 5.17 Species explaining the differences between spring 183

and autumn across all sites.

$\begin{array}{lll}\text { Table 5.18 Species explaining the differences between autumn } & 183\end{array}$

and summer across all sites.

Table 5.19 Morphologies explaining the similarities within 184

Spongy Hillocks across all seasons.

Table 5.20 Morphologies explaining the similarities within Broad 184

Gulley across all seasons.

Table 5.21 Morphologies explaining the similarities within Dogs 184

Leg across all seasons.

Table 5.22 Morphologies explaining the differences between 184

Spongy Hillocks and Broad Gulley across all seasons.

Table 5.23 Morphologies explaining the differences between Spongy Hillocks and Dogs Leg across all seasons.

Table 5.24 Morphologies explaining the differences between 185

Broad Gulley and Dogs Leg across all seasons.

Table 5.25 Morphologies explaining the similarities within 185 spring across all sites.

Table 5.26 Morphologies explaining the similarities within 185

Table 5.27 Morphologies explaining the similarities within 185

Table 5.28 Morphologies explaining the differences between 185 spring and summer across all sites.

Table 5.29 Morphologies explaining the differences between 185 spring and autumn across all sites.

Table 5.30 explaining the differences between autumn and 186

Table 6.1 PERMANOVA model design for the analysis of the uni- 196 and multivariate species data in response to the listed factors.

Table 6.2 Sponge species identifications from the study sites at 200 Hoga

Table 6.3 PERMANOVA results of the species assemblage data. 205

Table 6.4 O.T.U's which are important in explaining the 206 similarity within Sites across all years sampled.

Table 6.5 Species explaining the similarity across all sites in 213 2005

Table 6.6 Species explaining the similarity across all sites in 213 2006

Table 6.7 Species explaining the similarity across all sites in 213 2007

Table 6.8 Species explaining the similarity across all sites in 2008 
Table 6.9 Species explaining the similarity across all sites in

Table 6.10 Species explaining the differences between all sites in 2005 and 2006

Table 6.11 Species explaining the differences between all sites in 2005 and 2007.

Table 6.12 Species explaining the differences between all sites in 2006 and 2007.

Table 6.13 Species explaining the differences between all sites in 2005 and 2008.

Table 6.14 Species explaining the differences between all sites in 2006 and 2008.

Table 6.15 Species explaining the differences between all sites in 2007 and 2008.

Table 6.16 Species explaining the differences between all sites in 2005 and 2009.

Table 6.17 Species explaining the differences between all sites in 2006 and 2009.

Table 6.18 Species explaining the differences between all sites in 2007 and 2009

Table 6.19 Species explaining the differences between all sites in 2008 and 2009.

Table 6.20 Species explaining the similarity across all years in site $A$.

Table 6.21 Species explaining the similarity across all years in

Table 6.22 Species explaining the similarity across all years in

Table 6.23 Species explaining the differences between sites A and $B$ across all years.

Table 6.24 Species explaining the differences between sites A and $C$ across all years

Table 6.25 Species explaining the differences between sites B and $\mathrm{C}$ across all years. 
CHAPTER 1

GENERAL INTRODUCTION 
The biodiversity of marine ecosystems worldwide is declining (Roberts et al., 2002; Pandolfi et al., 2003; Jackson, 2008). Marine resource managers are trying to prevent the decline in biodiversity by attempting to preserve whole habitats in the form of marine reserves and Marine Protected Areas, as well as by developing species-specific conservation plans (Fahrig \& Merriam, 1994; Allison et al., 1998; Lubchenco et al., 2003). In order to protect a habitat, the community composition must be known along with how each element within it functions and interacts over different temporal and spatial scales.

Sponges are an ecologically important group in benthic marine communities (Sara \& Vacelet, 1973; McClintock et al., 2005). They have been a major component of the marine benthic fauna from the early Cambrian to the present day and are found across the world's benthic environments (Reiswig, 1974; Dayton, 1979; Alvarez et al., 1990; Diaz et al., 1993; Økland \& Økland, 1996; Bell \& Barnes, 2000a; Hooper et al., 2002; Bell \& Smith, 2004; Leys et al., 2004; Picton \& Goodwin, 2007).

Sponges have many functional roles within marine ecosystems, which have been recently reviewed and include: facilitating primary production; nitrification; silification; spatial competition through chemical and physical adaptation; stabilisation and erosion of the substrate or reef framework; alteration of the microflow dynamics of the water column; the filtration of the water and release of secondary metabolites; providing a food source; habitat; and increase survival of other species (Diaz \& Rützler, 2001; Wulff, 2001; Wulff, 2006a; Bell, 2007a; Bell, 2008). Despite the wide range of functional roles that sponges fulfill, and the steady increase in sponge ecological research since the 1970 's, far less is still known about sponge ecology compared with other phyla of similar sizes, even though they are known as a important component of marine benthic communities (Sara, 2004; Becerro, 2008).

Despite sponges being abundant and diverse component of benthic ecosystems they are still generally being ignored or only recorded as 'sponges' in many monitoring programmes across the world. This is due to time constraints of 
underwater research and sponges being difficult to work with due to the lack of identification keys, the difficulty of identifying them easily in the field, and the problems in their quantification (Rützler, 1978; Wulff, 2001). Morphological monitoring can therefore provide a cost effective method of monitoring the variability of sponge assemblages in areas where expertise, money and/or time are limiting factors for monitoring schemes. However species information is useful to be able to establish baselines and identify what changes are outside of the normal levels of variability (Bell \& Barnes, 2001; Bell et al., 2006; Bell, 2007b).

Documenting and describing biodiversity is a time consuming process that requires accuracy and patience, however, it is an essential prerequisite to conservation and management of marine resources, including sponge assemblages (Mace, 2004). Biodiversity can be defined in many ways but generally it encompasses:

Genetic variation - diversity of genes within a species. There is a genetic variability among the populations and the individuals of the same species.

Species richness - diversity among species within an ecosystem. This is the number of species within a particular habitat, region or ecosystem.

Ecosystem complexity - encompasses the variety of habitats that occur within a region, or the mosaic of patches found within a landscape.

Functional diversity - measures the number of functional groups in a community.

All aspects of biodiversity need to be taken into consideration when designing monitoring schemes to protect benthic communities (Bell, 2007a).

There is now a growing body of literature on global sponge species diversity with approximately 8,500 valid described species (Hooper \& van Soest, 2002; van Soest et al., 2008). However, this number is likely to double as marine habitats, such as the deep sea are explored further, as well as describing the cryptic and encrusting sponge fauna, which is probably equally diverse as the 
macrobenthic fauna in better known shallow water habitats (van Soest, 2007; van Soest et al., 2008).

Areas such as the United Kingdom, Mediterranean and Caribbean have relatively well-known sponge assemblages, which reflects the relatively long period of time scientists have worked in these areas (Balsamo Crivelli, 1863; Bowerbank, 1864, 1866, 1874; Bowerbank \& Norman, 1882; Arndt, 1935; de Laubenfels, 1953; Labate, 1964; van Soest, 1978; Alcolado, 1979; van Soest, 1980, 1984; Bibiloni et al., 1989; Corriero, 1989; Hubbard, 1990; Ghiold et al., 1994; Borg \& Schembri, 1996; Lehnert \& Soest, 1996, 1998; Pansini \& Longo, 2003; Ackers et al., 2007; van Soest et al., 2008). Other places have moderately well known faunas, such as New Zealand, New Caledonia and Japan (Bergquist, 1968, 1970; Bergquist \& Warne, 1980; Hoshino, 1981a, b; Bergquist \& Fromont, 1988; Bergquist, 1996; Lévi et al., 1998; Kelly, 2007). This difference between geographic regions is due to relatively recent exploration and/or research effort being concentrated in a small area of a region, for example, the majority of sponge diversity work in New Zealand being concentrated in the waters close to Auckland on the North Island (Bergquist, 1961; Ayling, 1979; Battershill \& Bergquist, 1990). Other regions have poorly known sponge faunas, such as Indonesia, Papua New Guinea and other parts of the Indo-Malay archipelago and most of our knowledge is from the 1900s and a few recent expeditions, (Sollas, 1902; Dragnewitsch, 1906; van Soest, 1989, 1990; Pulitzer-Finali \& Pronzato, 1999). It is however, well known from studies on other animal groups that this region is a marine biodiversity hotspot, and there is no reason to suggest that this is different for sponges (Roberts et al., 2002; Lind et al., 2007; Scaps et al., 2007; Allen, 2008). As biodiversity includes ecosystem complexity it is important to consider species richness across different spatial and temporal scales to understand how to 'conserve' biodiversity at multiple spatial scales and through time.

There have been studies on sponges at all levels including: genetic diversity (Solé-Cava et al., 1991; Boury-Esnault et al., 1992; Solé-Cava et al., 1992; SoléCava \& Boury-Esnault, 1999; Miller et al., 2001; Wörheide et al., 2008) 
biochemical variability (Page et al., 2005; Abdo et al., 2007), morphological variability of a particular species (Meroz-Fine et al., 2005), distribution patterns of a particular species or genus (Boury-Esnault et al., 1993; Kelly, 2003; Usher et al., 2004), abundance and diversity within a particular habitat - alpha diversity (Barnes, 1999; Bell, 2007a; Carballo \& Nava, 2007; Wulff, 2008), geographic area - beta diversity (Adjeroud \& Salvat, 1996; Hooper et al., 2002; Kefalas et al., 2003; Bell \& Smith, 2004; Duckworth et al., 2008), across different geographic regions - gamma diversity (Wilkinson, 1987; Wilkinson \& Cheshire, 1990; Wulff, 1996; Bell, 2007a), and comparing the spatial variability of sponge assemblages at different scales (Hooper et al., 2002; Barnes \& Kuklinski, 2004).

The factors influencing spatial distribution include physical factors such as: depth (Wilkinson \& Evans, 1989; Duckworth et al., 2004; Cleary et al., 2005), water flow (Leichter \& Witman, 1997; Duckworth et al., 2004), temperature (Riisgard et al., 1993; Barnes, 1999), light intensity (Alcolado, 1994), sedimentation (Sara \& Vacelet, 1973; Wilkinson \& Vacelet, 1979; Zea, 1994; Ilan \& Abelson, 1995) and salinity (Fell et al., 1989; Leamon \& Fell, 1990; Barnes, 1999).

Biological factors influence sponge spatial distribution patterns include: predation (Wulff, 1994, 1995b, 1997b, 2000) mutualistic and symbiotic associations (Rosell \& Uriz, 1992; Arillo et al., 1993; Wulff, 1997a), concentration and type of plankton (Pile, 1996; Ribes et al., 1999; Ribes et al., 2003), spatial competition (Jackson, 1977; Aerts, 1998; Fagerstrom et al., 2000) and disease - (Smith, 1941; Galtsoff, 1942; Pronzato, 1999; Wulff, 2007).

Any sponge assemblage has a multitude of factors acting upon it and the sponges also have a functional role within the marine ecosystem, and therefore changes in sponge assemblages can lead to potential cascading effects on other components of marine systems. Spatial variability is often a snapshot and the current composition of an assemblage is the result of the history of that particular area. The species composition of a sponge assemblage will have traits that tend to stabilise the interactions between species competing for space and 
other resources (Butler \& Chesson, 1990). Biological factors may be more important than physical factors in determining tropical sponge assemblage structure in the Caribbean (Wulff, 1997b, 2000), however, physical factors have been found to be more important in the North Eastern Atlantic and Eastern Pacific (Bell \& Barnes, 2000a; Bell \& Barnes, 2003a; Carballo, 2006).

Some studies have tried to limit the number of potential factors explaining sponge assemblage variation to elucidate larger patterns between regions, such as using very cold environments under ice, caves, sea lochs or the underside of boulders (Dayton, 1979; da Costa et al., 1992; Benedetti-Cecchi et al., 1997; Bell \& Barnes, 2000b, c; Bell, 2007a). These studies, which limit the variability between transoceanic sites have the potential to identify common factors that structure sponge assemblages across different biogeographic regions (Bell, 2007a).

Although algae dominates the biomass of subtidal $(<12 \mathrm{~m}$ deep $)$ reef communities around mainland New Zealand, amongst the encrusting invertebrate groups, sponges have been reported as the largest contributor to total biomass in many locations, for example, at Raglan on the North west coast of the North Island and Chalmers near Dunedin on the South Island (Shears \& Babcock, 2007). There are large areas around New Zealand, which have not yet been surveyed fully for sponges. For example, at Pariokariwa reef, North Taranaki a preliminary survey reported 57 sponges species, of which only 17 were given likely or confirmed species names (Battershill \& Page, 1996). Despite this earlier research, there have been no peer reviewed publications about Pariokariwa reef even though it is designated a marine reserve partially because it is considered one of the 'top sponge spots' in the world (Battershill pers. com.).

In order to understand the factors explaining the spatial patterns of sponge diversity it is important to also consider temporal diversity. The majority of factors which have an effect on sponge assemblages vary over different temporal scales such as day length, tidal regime (Bergquist \& Tizard, 1967), a 
fairly regular pattern such as sea temperature (Harsha et al., 1983; Burlando et al., 1992; Maldonado \& Young, 1996) or less predictable factors, such as human impacts or large environmental effects such as hurricanes (Wilkinson \& Cheshire, 1988; Wulff, 1995a; Perez et al., 2005; de Voogd et al., 2006; Wulff, 2006a).

Variability of physical and biological processes operate over a range of temporal scales from milliseconds to millions of years (Clarke, 2001). In order to understand benthic ecosystems, a long-term perspective (at least 5 years) is important (Dye, 1998; Carballo et al., 2008), yet most research is carried out over a short time period (Likens, 1989). Most studies of sponge assemblages have used a single sampling interval in one location and there have been relatively few studies examining the same sponge assemblage over more than a year. Research from the Mediterranean has shown slow growth within sponge assemblages with no clear seasonality and tissue regression only for some species, while the abundance of different sponge species remained similar throughout an eight year study (Sara, 1970; Pansini \& Pronzato, 1985). Similar results have also been reported from the Atlantic (Fowler \& Laffoley, 1993; Hiscock, 1994) and the Caribbean (Hughes, 1996). In fact, even though the overall abundance of sponge individuals and percentage cover within assemblages remained relatively constant in the Caribbean, the actual individual sponges were very variable suggesting recruitment and mortality were in equilibrium for these assemblages (Hughes, 1996). However, more recent work in the Atlantic, Caribbean and Pacific suggests that more rapid changes in sponge assemblages are common and recruitment and mortality do not happen at the same rate (Bell et al., 2006; Wulff, 2006b; Carballo et al., 2008).

There have been studies of seasonal growth rates/abundance of sponges at the species level in most marine benthic environments (Burton, 1949; Stone, 1970; Bryan, 1973; Ayling, 1983b; Dayton, 1989; Contini, 1995; Garrabou \& Zabala, 2001; Tanaka, 2002). In Northern New Zealand it was found that thinly encrusting sponges grow at a slow, but measurable rate, unless they are 
damaged and then they have a rapid regeneration rate over 200 times that of the normal growth rate (Ayling, 1983b). The rapid regeneration rate would help the sponge maintain the boundaries of their patches (Ayling, 1983a). Regeneration is integral to the survival of sponges and regenerative growth is normally a thin protective layer to 'seal up' the damage and is not tissue as thick as with normal growth processes. Growth and sexual reproduction are impaired during recovery as resources are diverted to heal the pinacoderm (Henry \& Hart, 2005).

Regeneration rates were also measured for sponges of varying morphology (including massive, papillate, branching and encrusting) in temperate New Zealand waters to test their suitability for aquaculture. The sponge explants were left to grow at $5 \mathrm{~m}$ and $10 \mathrm{~m}$ and the explants had better regeneration and growth rates at $10 \mathrm{~m}$, which is also the depth of their normal habitat. The explants also survived and grew at a better rate if they were transplanted during winter and it was suggested this promoted healing due to a lower exposure to UV light (Duckworth et al., 1997; Duckworth et al., 2004). UV light has been shown to have a detrimental effect on sponge growth and survivorship, especially if the sponge does not normally have a high exposure to UV than in its normal habitat (Wilkinson \& Vacelet, 1979; Jokiel, 1980). The faster healing rate of the pinacoderm (surface of the sponge) was attributed to lower temperatures (Duckworth et al., 1997). This seems to contradict metabolic laws unless other factors are involved as seasonal increases in water temperatures could be predicted to enhance regenerative capacity due to higher metabolic rates and thus higher growth rates.

The growth rates of common sponges Hymeniacidon and Halichondria were measured in temperate southern UK waters and they tend to grow and fuse with other sponge patches in summer and regress and fragment in winter (Burton, 1949; Stone, 1970). A similar pattern was also found in New England, USA for Hymeniacidon (Hartman, 1958). Many demosponges across temperate and tropical regions show seasonal growth in summer followed by little growth or regression in winter (Elvin, 1976; Fell \& Levandrowski, 1981; Barthel, 1986; 
Turon et al., 1998; Garrabou \& Zabala, 2001; Tanaka, 2002; McMurray et al., 2008). Other demosponges however, do not appear to have any seasonal growth patterns (Ayling, 1983b; Hoppe, 1988; Tanaka, 2002). Growth rates of calcareous sponges in the UK have been shown to increase in winter and colonies die off in summer, the opposite pattern to many of the demosponges (Dendy, 1914; Orton, 1914; Burton, 1949; Johnson, 1979).

Although there has been some conducted in New Zealand on spatial and temporal variability of sponge assemblages, it has been carried out at the species rather than assemblage level and only in northern New Zealand. There are still no studies on sponges, comparing spatial and temporal variation concurrently at different spatial scales. It is vital for future research to focus on broad-scale investigations so that the impact of global change can be determined and predicted, and suitable methodology can be developed so that sponges can be easily included in monitoring schemes for habitat management.

This research in this thesis investigates the spatial and temporal variability of sponge assemblages using examples from temperate and tropical sponge dominated sites in Wellington, New Zealand, South East Sulawesi, Indonesia, and Wales, UK. Chapter 2 reviews the current knowledge of broad sponge biodiversity patterns in New Zealand coastal waters and how they relate to the current conservation management schemes. Chapter 3 focuses on the Wellington South Coast, New Zealand in order to develop a higher resolution of the spatial variability of sponge assemblages including abundance and percentage cover data rather than the coarse presence only data used for the New Zealand overview in Chapter 2. Chapter 4 builds upon the spatial variability of Chapter 3 of the Wellington South coast and investigates the seasonal temporal variability of the sponge assemblages in the area and which environmental factors can be related to the patterns of variability. Chapter 5 provides a contrasting temperate example of the spatial and temporal variability of sponge assemblages in the Skomer Marine Reserve, Wales, U.K. This chapter also investigates if morphological groups of sponge assemblages show similar patterns in relation to seasonal variability to the species data. In 
Chapter 6, sponge assemblages from vertical walls off Hoga Island, SE Sulawesi, Indonesia were used to provide a tropical example of spatial and temporal diversity on an annual scale. Chapter 6 also compares the patterns the composition of species diversity and abundance and how the diversity is partitioned temporally and spatially. The thesis concludes with a general discussion and synthesis of the research to compare the different monitoring techniques investigated during this thesis and spatial and temporal sponge assemblage patterns of the inter-oceanic examples.

Overall aims are:

1. To understand the scales of spatial variation of sponge assemblages using the following case studies: New Zealand (Regional and Local); Wellington, NZ, Skomer, UK and Hoga, Indonesia (Local)

2. To understand the scales of temporal variation of sponge assemblages using the following case studies: Wellington, NZ and Skomer, UK (Annual and Seasonal) and Hoga, Indonesia (Annual).

3. To develop sponge monitoring guidelines for a range of spatial and temporal scales.

This thesis will investigate the hypothesis that sponge assemblages will show similar shifts of abundance and diversity in relation to biological and/or environmental factors at a range of locations around the world. Therefore it would be possible to predict future changes/ try to understand which factor is most responsible for a rapid increase or decline in biodiversity. 


\section{CHAPTER 2}

SPATIAL PATTERNS OF SPONGE BIODIVERSITY IN NEW ZEALAND COASTAL WATERS 


\subsection{ABSTRACT}

Biodiversity is not distributed evenly around the world and determining why some areas are so-called 'hotspots' and others contain low diversity is a fundamental objective for ecologists and biogeographers. However, patterns can be hard to predict in the marine environment as many habitats have been poorly sampled and some phyla have been poorly described and studied. In this study, 4735 Porifera records from New Zealand shelf waters encompassing 805 described and undescribed sponge species within 215 genera were used to investigate spatial patterns of sponge diversity. There was a similar relationship for the spatial distribution of species and genera level data for the subset used from harbours of New Zealand. There was a clear shift in the biodiversity of sponge assemblages with depth; and a combination of depth and location was responsible for about 50\% of the variation in the Average Taxonomic Distinctness (AvTd) and the Variation in Taxonomic Distinctness (VarTD) of the sponge assemblages around New Zealand. There were limited data available from some of the areas, in particular from the west coast of the South Island and most data has been collected from point sources therefore it is vital to expand the areas surveyed for a more detailed picture. 


\subsection{INTRODUCTION}

Maintaining biodiversity is vital for preserving ecosystem function and stability, as well as sustaining the economic potential of species, habitats and assemblages (Hooper et al., 2005; Worm et al., 2006). Biodiversity is defined as all life on earth, including plants, animals, and microbes-at scales ranging from genetic diversity within populations, to species diversity, to community diversity across landscapes (Sala et al., 2000). Broad-scale patterns of terrestrial biodiversity are reasonably well known for many phyla (Gaston, 2000; Jetz et al., 2009). However, the factors driving the patterns of marine biodiversity are not as well known and the patterns often contrast with those of terrestrial diversity (Rutherford et al., 1999; Worm et al., 2005). Terrestrial biodiversity tends to increase towards the equator and in more productive regions, for example forests in comparison to deserts. Sea surface temperature is one of the few environmental factors that correlates with the distribution patterns of a wide variety of taxa from foraminifera to sharks (Tittensor et al., 2010). Most marine taxa increase in diversity as sea temperature increases, apart from a few exceptions which show the reverse pattern such as seals. Biodiversity is not distributed evenly around the world and determining why some areas are so-called 'hotspots' and others contain very low diversity is a fundamental objective for ecologists and biogeographers (Gaston, 2000). However, patterns can be hard to predict in the marine environment as many habitats have hardly been sampled and some phyla have been poorly described and studied (Boakes et al., 2010; Costello et al., 2010). Therefore, taxonomy, species distribution data and natural history are basic requirements for understanding the patterns of biodiversity, monitoring and conservation management (May, 1990; Balmford \& Gaston, 1999; Dayton, 2003; Pressey, 2004; Wheeler, 2004; Teder et al., 2007; Boero, 2010; Costello et al., 2010).

The availability of sufficient and suitable data, such as full species lists over areas with equal sampling effort and methodology, can often restrict the spatial 
extent over which patterns of biodiversity can be assessed (Gaston, 2000). Therefore surrogates are often used in order to provide an indication of spatial variability in biodiversity and designate important areas for conservation management and protection (Margules \& Pressey, 2000; Pressey, 2004). Surrogates for biodiversity are normally environmental or taxonomically-based measures. Environmental surrogates are usually based on a combination of physical and biological data. They can be subdivided into two types: those based on discrete classes (often referred to as ecological classifications or land types); and those where continuous data are analysed directly in the areas selected (Araújo et al., 2001; Faith, 2003). Taxonomic surrogates are predominantly based on biological data, include the use of well-known groups of species, such as birds or marine molluscs, and are often extrapolated geographically using statistical techniques (Garson et al., 2002; Terlizzi et al., 2009). In a New Zealand and worldwide study it was found that genera or family level data for molluscs could be used to as a surrogate for species diversity patterns at a regional level (Terlizzi et al., 2009).

New Zealand has the fifth largest exclusive economic zone (EEZ) in the world spanning $30^{\circ}$ latitude with a coastline of 15-18,000 km long and an area of almost 4.2 million $\mathrm{km}^{2}$. The EEZ is an area known to be rich in resources, including over 17,000 known living species, even though over $50 \%$ of the region is biologically uncharted (Gordon et al., 2010). The sustainable management of the EEZ is a challenge for New Zealand's small population equating to roughly $1 \mathrm{~km}^{2}$ of ocean per New Zealander.

New Zealand's marine biodiversity has been shaped by temporal and physical isolation, geological history and the complexity of present day oceanographic patterns and processes (Gordon et al., 2010). Zealandia (New Zealand's regional continental land mass) split away from Antarctica and Australasia in the late Cretaceous approximately 83-100 million years ago (Mortimer et al., 2006). Sea level changes, tectonic activity and rotational displacements over the subsequent 50 million years led to huge fluctuations in the area of land above 
sea level, until the majority was submerged about 23 million years ago (Landis et al., 2008). By 5 million years ago the Southern Alps had risen to form a continuous mountain chain, which led to huge quantities of sediment being eroded onto the continental shelf and into the deep sea. In fact, currently, on average 209 million metric tonnes of sediment reaches the sea from the mountains of New Zealand each year, which is $1 \%$ of the global amount of sediment reaching the oceans from only $0.2 \%$ of the land area (Griffiths, 1979; Pickrill, 1993). The Pleistocene glaciations over the last 2.6 million years and in particular the last 0.7 million years have led to multiple sea level fluctuations, which have influenced New Zealand's current day shelf biota (Suggate, 1990). New Zealand forms the southwestern boundary to the Pacific Ocean with the Pacific crustal plate to the east and the Australasian plate to the west as part of the "ring of fire". This means that Zealandia is still active tectonically with areas of uplift and subsidence and on and offshore volcanic activity. The Kermadec ridge is $2,500 \mathrm{~km}$ from New Zealand to Tonga is the longest submarine arc on the planet, which has areas of hydrothermal vents and seamounts which support chemosynthetic ecosystems (de Ronde et al., 2007; Clark et al., 2010) (Bachraty et al., 2009). Along the edges of the continental shelf there are also areas of instability where there are cold seeps of methane that also support chemosynthetically-based communities (Baco et al., 2010).

The oceanography of New Zealand's waters is complex as the land forms the boundary between the sub tropical convergence, which is the subtropical water approaching form the north and subantarctic water from the south with their own distinctive temperature and salinity characteristics (Heath, 1985; Carter et al., 1998). The major currents divide as they reach the continental shelf creating many smaller currents and eddies bringing oceanic water into the coastal zone (Fig. 2.1). These coastal currents are then further modified by the complex topography of the land (islands, canyons and headlands) and the temporally variable waves, wind and tides. 


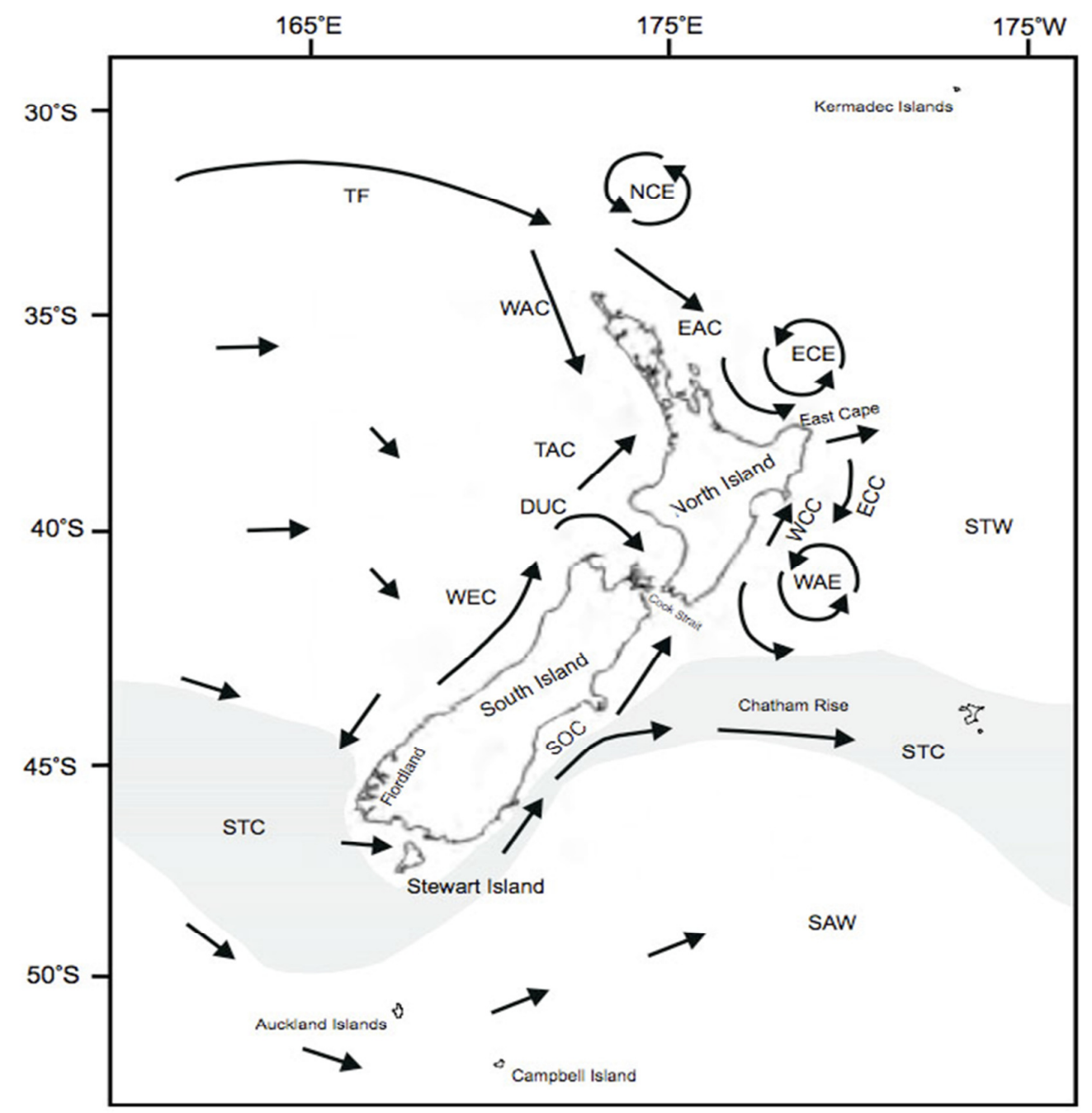

Fig. 2.1 Major coastal current systems of New Zealand. DUC, D'Urville Current; EAC, East Auckland Current; ECC, East Cape Current; ECE, East Cape Eddy; NCE, North Cape Eddy; SOC, Southland Current; SAW, Subantarctic water; STC, Subtropical Convergence; STW, Subtropical water; TAC, Tasman Current; TF, Tasman Front; WAC, West Auckland Current; WAE, Wairarapa Eddy; WCC, Wairarapa Coastal Current; WEC, Westland Current (redrawn after Heath (1985) and Carter et. al (1998).

The tides around New Zealand are only 1-2 $\mathrm{m}$ in range, have a semi-diurnal regime and the seasonal patterns of phytoplankton blooms are typical of temperate waters (Chang et al., 2003).

Numerous attempts have been made to try and classify the New Zealand marine environment using physical and/or biological data (Moore, 1949; Knox, 1975; Nelson, 1994; Walls, 1995; Francis, 1996; Apte \& Gardner, 2002; Anon, 2005; Snelder et al., 2007; Shears et al., 2008; Beaumont et al., 2010; Gordon et al., 
2010). The New Zealand biodiversity strategy 2000 aims to create a network of Marine Protected Areas (MPA's) in order to protect a proportion of all the marine habitats present in New Zealand waters (Anon, 2000). Bioregions cover relatively large areas of water, and contain characteristic, geographically distinct assemblages of natural communities and species (Anon, 2005). The Department of Conservation (DOC) is using a Coastal Biogeographic Regional Classification scheme to divide coastal waters less then $200 \mathrm{~m}$ deep into fourteen coastal biogeographic regions including nine mainland regions, three offshore Island archipelagos, and two inner island bioregions (Fig. 2.2) (Anon, 2005).

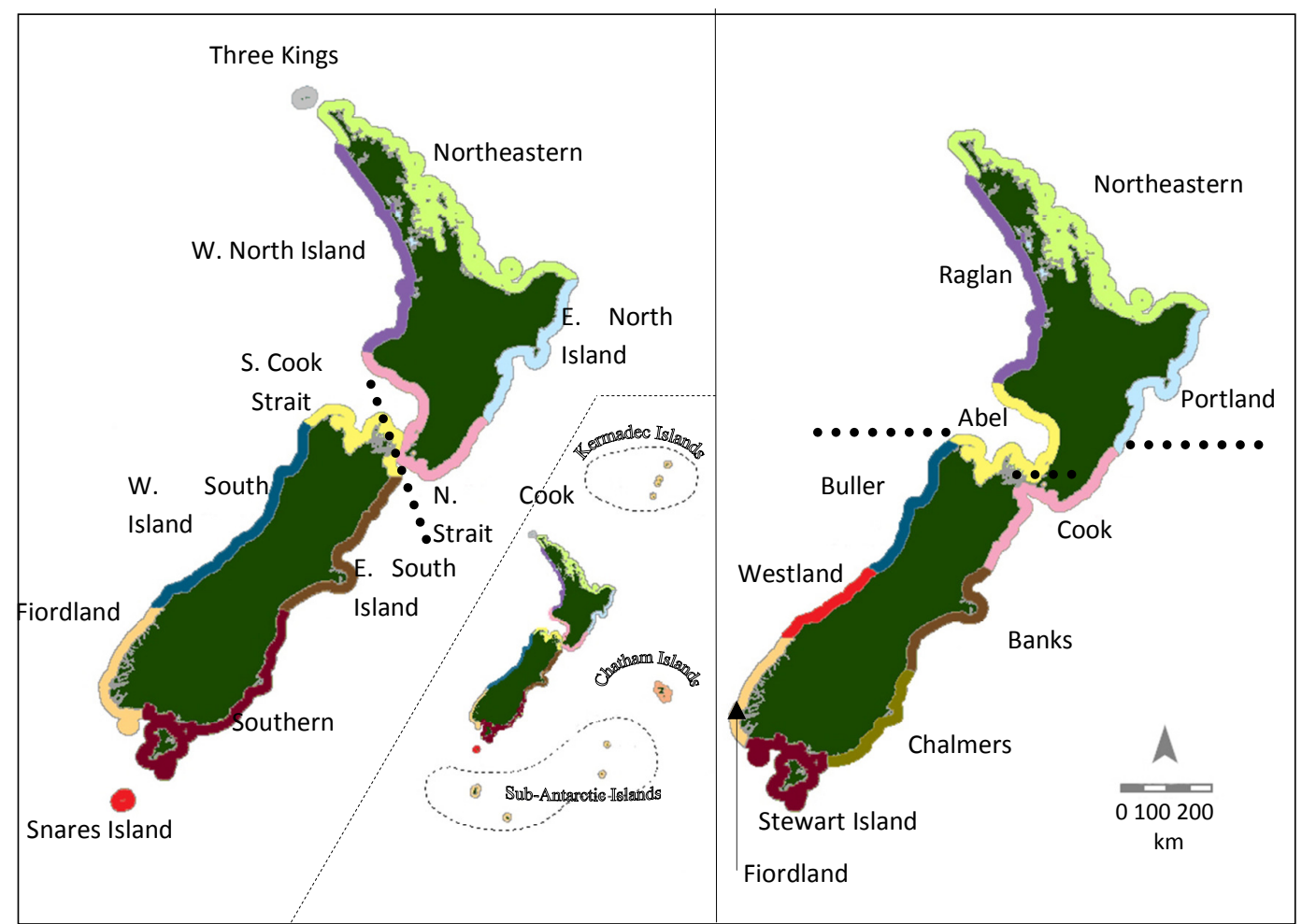

Fig. 2.2 Current New Zealand biogeoclassification schemes. The map on the left depicts New Zealand's proposed 14 coastal bioregions used by the Department of Conservation and the Ministry of Fisheries (Anon, 2005). These include New Zealand's offshore Island archipelagos (inset). The map on the right depicts the recommended bioregions according to Shears et al. (2008). They include within their boundaries inner coastal islands, except for the Three Kings and Snares. The different colours signify the boundaries between the different bioregions. The dotted lines in each case depict the predicted bioprovince break between the North and South Islands. 
Since the Cape Rodney - Okakari Point marine reserve was established in 1975 as one of the world's first no take marine reserves, there are now 33 MPA's protecting about $7.6 \%$ of the territorial sea area (TS - the 12 nautical mile from shore zone), however, these are not spread evenly between bioregions. Approximately $99 \%$ of this protected area is composed of just two marine reserves around isolated offshore island groups (Auckland Islands and the Kermadecs). Marine Reserves can, according to current law (Marine Reserves Act 1971) only be created within the TS (MoAF/DoC, 1971). Nevertheless, since 2001 there have been fisheries closures for trawling around 19 seamounts and since 2007 there have been an additional 17 benthic protection areas in deeper water which protect approximately $30 \%$ of the EEZ from deep sea trawling and dredging activities (Clark \& O' Driscoll, 2003; Gordon et al., 2010).

Shears et. al. (2008) used macroalgal assemblage and mobile invertebrate surveys to divide mainland New Zealand into eleven bioregions within two biogeographic provinces. These only included shallow mainland coastal areas (0-12 $\mathrm{m}$ depth and within $150 \mathrm{~m}$ of the intertidal reef). While this work was effective for defining bioregions for algae and mobile invertebrate assemblages in sub-tidal waters it did not take into consideration assemblages deeper than $12 \mathrm{~m}$ and other taxa, such as the sessile invertebrates.

Bryozoans have been investigated as a potential indicator taxon for quantifying biodiversity around New Zealand (Rowden et al., 2004). Rowden et al. (2004) proposed that the bryozoan assemblage taxonomic distinctness was mainly split by depth ( $<200 \mathrm{~m}$ and two deep sea communities $)$ and there were hotspots of diversity at the Three Kings and Foveaux Strait and seamounts on the Chatham Rise. As there is a clear split in biodiversity at the shelf edge it is important to explore the biodiversity patterns within the $0-200 \mathrm{~m}$ depth boundaries. A first attempt to describe the spatial distribution of the complete biodiversity around the New Zealand coastline has been recently attempted using a combination of habitat and biological data, however, this did not go as 
far as relating the patterns of biodiversity to the current DOC bioregion scheme (Beaumont et al., 2008; Beaumont et al., 2010; Gordon et al., 2010).

Although algae dominates the biomass of shallow subtidal $(<12 \mathrm{~m}$ deep) reefs around mainland New Zealand, amongst the encrusting invertebrates, sponges have been reported as the largest contributor to total biomass in many locations (Shears \& Babcock, 2007). There are 1,435 known species of Porifera in the New Zealand EEZ and it is considered to be one of the better known animal groups, although still less than $50 \%$ of the known species are described (Gordon et al., 2010). Typical habitats that have rich sponge assemblages in New Zealand are rocky subtidal reefs, underneath rocks and boulders, and on vertical and overhanging bedrock, especially in channels, crevices, caves and gulleys (Kelly et al., 2009; Berman \& Bell, 2010) (Fig. 2.3). Extensive sponge gardens have also been reported in deeper waters in a variety of habitats from seamounts to soft sediment plains (Cryer et al., 2000; Kelly, 2000, 2003; Kelly et al., 2009).

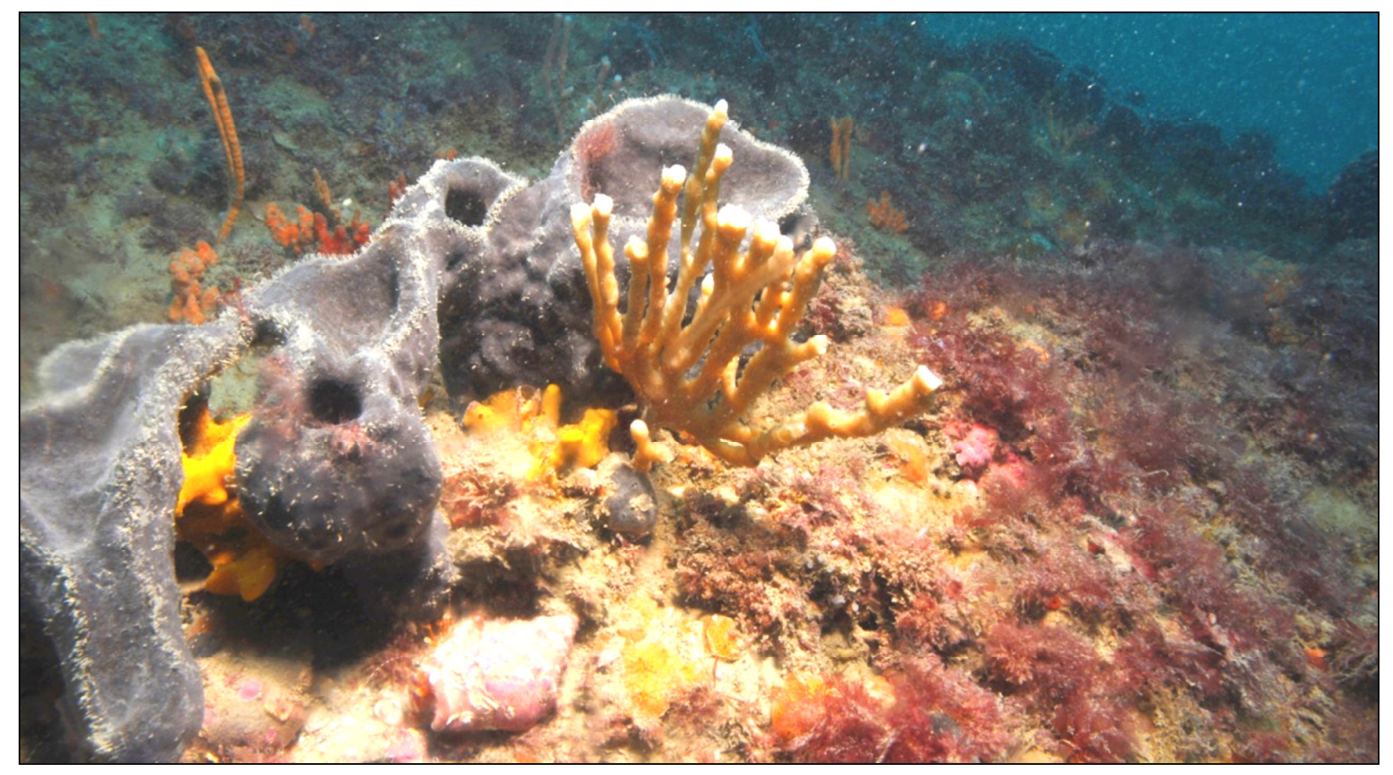

Fig. 2.3 A typical sponge garden assemblage at $18 \mathrm{~m}$ depth at Parininihi MPA, Taranaki. 
As sponges are found over a wide depth, habitat and latitudinal and longitudinal range they are a suitable model taxon to compare ecological processes over a wide range of spatial scales (Bell \& Carballo, 2008). Variation in sponge diversity, distribution and abundance is likely to influence other benthic organisms due to the dominance of sponges in hard benthic environments and the important functional roles that sponges fulfill. (Pang, 1973; Rützler, 1975; Wulff \& Buss, 1979; Corredor et al., 1988; Ribeiro et al., 2003; Bell, 2007a; Bell, 2008). Sponge diversity is influenced by physical and biological factors which includes: depth (Wilkinson \& Trott, 1983; Wilkinson \& Evans, 1989; Roberts \& Davis, 1996), light (Wörheide et al., 2005), sedimentation (Bell \& Barnes, 2000b, c), wind speed (Carballo et al., 2008), habitat stability (Carballo \& Nava, 2007), habitat availability (Sara, 1970; Bell \& Barnes, 2003a, b), predation (Wulff, 2000), human settlement (de Voogd et al., 2006) and competition (Ayling, 1981).

The main limitations of using sponges as a model taxon are the limit of taxonomic knowledge in New Zealand. Although almost 1500 sponge species are known from New Zealand waters almost 1000 of those sponge species have not yet been described, and there is only one professional sponge taxonomist in the region working her way through the samples (Gordon et al., 2010). Underestimates of global sponge diversity have resulted primarily from a lack of comprehensive locality-specific sampling and difficulties in classification. In particular many sponge genera lack an adequate range of taxonomic characters for species differentiation, and morphological and skeletal characters are plastic (Bergquist \& Kelly-Borges, 1991; Kelly et al., 2009). Also, although many species recorded from New Zealand waters are listed as cosmopolitan, it is thought that over $95 \%$ of sponge species within New Zealand waters are actually endemic (Kelly et al., 2009). Most of the published knowledge of New Zealand sponge biodiversity is in the form of species lists for an area or older collections with no information on the abundance or likely species ranges (Kelly et al., 2009). There is however a large database of sponge specimens and records kept within the 
National Institute of Water and Atmospheric Research (NIWA) which provides most of the information on sponge biodiversity in New Zealand (M. Kelly pers. com). Therefore it would be useful if sponge biodiversity could be examined at a regional scale using genera level data rather than species level due the high number of species which are currently only identified to genera level, misidentified at species level, and recorded in biological surveys at genera level only (Battershill \& Page, 1996; Berman \& Bell, 2010). The potential use of coarser levels of taxonomic resolution to detect patterns of biological variability using presence/absence data has been explored and found valid for molluscan assemblages therefore it is important to elucidate if this is the case for other phyla, especially where there is a lack of taxonomic expertise, for example sponges (Terlizzi et al., 2003; Terlizzi et al., 2009).The aims of this chapter were to understand:

1. Whether genera level sponge data can be used as surrogates in understanding patterns of diversity along a depth and latitudinal gradient within New Zealand.

2. To test wether the bioregional hypothesis proposed by Shears et. al. (2008) and DOC are applicable to sponges.

3. To determine any biogeographical patterns in the distribution of sponges in the coastal waters of New Zealand using data from upto $200 \mathrm{~m}$ deep.

a. Are these similar to that of Shears et. al. (2008) and/or DOC or neither

b. Locate any boundary or transition zones between the hypothesized bioregions of either Shears et. al. (2008) and/or DOC.

4. To ascertain patterns of sponge relationships and biodiversity hotspots at genus (and species) level of diversity. 


\subsection{METHODS}

\subsubsection{DATA SOURCES}

The main data source for Porifera records for this study was the NIWA Porifera database, which has been assembled over the last twelve years as part of ongoing NIWA research voyages studying the ecology of seamounts and other coastal expeditions (Kelly unpub.). This database builds on the previous work by Dame Patricia Bergquist and historical and recent survey records for the EEZ. This was supplemented by additional surveys of the literature for marine reserves (see chapter 3) (Davidson, 1991; Jones \& Garrick, 1991; Davidson \& Brown, 1994; Battershill \& Page, 1996; Berman \& Bell, 2010), and recent collections and identifications by the author from the Pelorus Sound, Marlborough, Parininihi marine reserve, Taranaki, the Wellington South Coast and White Island, Bay of Plenty. Porifera voucher samples held at the University of Victoria, Wellington and at the University of Otago in Dunedin were also reexamined. Sponge taxonomic names have changed quite frequently over the years so all of the sponges were aligned with the names and taxonomic order stated in the World Porifera Database (WPD), which lists all recent sponges as part of the World Register of Marine Species (WoRMS) (van Soest et al., 2008). Sponges can be cryptic and many of the species encountered during surveys have only been classified to genus level, therefore for the main data analysis classifications were at genera level, which was defined as the operational taxonomic unit or O.T.U. (see 2.3.3 Data analysis for methodology to test if this approach is valid). The NIWA Porifera database and the other sources listed above were combined to create the master taxonomic list. This list is not included within this chapter as it contains unpublished data from M. Kelly which is owned by NIWA and therefore not publicly available. 


\subsubsection{STUDY AREA}

New Zealand's geological history, physical, geological, oceanographic and biological setting, has been recently reviewed (Gordon et al., 2010). New Zealand's mainland is defined as territorial waters within $12 \mathrm{~nm}$ of the North, South and Stewart Islands and excludes the offshore islands such as the Auckland and Chatham Islands. The New Zealand EEZ region for this study was defined by the NIWA standard charting area (latitude $24^{\circ} \mathrm{S}$ to $57^{\circ} 30^{\prime} \mathrm{S}$, longitude $157^{\circ}$ E to $167^{\circ} \mathrm{W}$ ), which includes the New Zealand 200 nautical mile EEZ including the offshore islands and surrounding extra territorial waters to $200 \mathrm{~m}$ depth. This depth was chosen as the limit as it extends to the edge of the continental shelf and previous work by Rowden et al. (2004) has found that the biodiversity patterns for bryozoans have distinctive shelf $(0-200 \mathrm{~m}$ assemblages) and two deep sea assemblages. Certain areas within the EEZ (for example, the Kermadec Islands and the West Coast of the South Island) have been excluded from the study as there were insufficient data in order to draw inferences about biodiversity patterns. Although the taxonomic distinctness indices are largely independent of sample size, there can be a small bias in the results leading to an underestimate of their values with very small species lists (Clarke \& Warwick, 1998; Pienkowski et al., 1998; Clarke \& Warwick, 2001b). Therefore only data sets with ten or more Operational Taxonomic Units (O.T.U.'s) were used in a similar manner to Rowden et al. (2004)

\subsubsection{SPECIES AND GENERA LEVEL ANALYSIS}

In order to elucidate if using genera-level data would provide enough resolution to deduce regional-scale patterns (using the predefined bioregions), a subset of data from the Porifera database was used to test the hypothesis that there would be no significant difference between the assemblage patterns of the data 
using the species level or genera level data. Ten data sets from port data surveys (included within the NIWA Porifera database) that had more than ten genera were chosen for this purpose (Inglis et al., 2005b, c, a, d, e, f; Inglis et al., 2008a; Inglis et al., 2008c; Inglis et al., 2008b; Inglis et al., 2008e, d). The reason these data were chosen as a subset was because a similar sampling effort was used for all of the surveys, the surveys took place at different locations all over New Zealand in similar habitats (scrapings off pilings) and all the identifications were made to species level by the same taxonomist (M. Kelly). The limitations of these data sets are that ports are often the first place alien species arrive and therefore are not always considered typical of the biodiversity of the area. Cranfield et al. (1998) listed nine sponge species considered to be adventives in New Zealand ports and harbours. However, all of the Porifera listed as likely to be invasive species are from genera in which species differentiation is extremely complicated owing to a lack of definitive characters within the genera (Kelly et al., 2009). Also this part of the study is examining the relationship between species level and general level data not looking for the differences in biodiversity between the ports or spatial variation.

Biodiversity indices were calculated for each port sample including Species/Genera richness, Average Taxonomic Distinctness (AvTD or $\Delta^{+}$) and the Variation in Taxonomic Distinctness (VarTD or $\Lambda^{+}$). Taxonomic distinctness quantifies diversity as the relatedness of the species within a sample, based on the distances between species in a classification tree (Clarke \& Warwick, 1998). AvTD is the mean of all species-to-species distances through the tree for all pairs of species within a sample, and represents the taxonomic breadth of the sample. A large value of AvTD indicates broad taxonomic diversity in the sample. A sample having only multiple species within a single genus will have lower taxonomic distinctness than a sample having multiple species from different families.

Here $\omega$ is the branch length between species pairs and $S$ is the number of observed species in the sample. 


$$
\Delta^{+}=\left[\sum \sum_{i<j} \omega_{i f}\right] /[S(S-2) / 2]
$$

For simple linear scaling $\omega=100$, which is the maximum distance through the taxonomic tree for the most distantly related species. When the branches are unweighted every step between taxonomic levels is considered to be equal. Standard Linnaean classification was used that corresponds to the WPD (van Soest et al., 2008). However, all taxa are not defined to the same level of detail. For example, some species had subfamilies while others did not or had suborders. Therefore the branch lengths were weighted by the number of species, within a classification at that level. For example, all the species recorded had a classification at genera level but only $18 \%$ have a subgenera classification (Table 2.1).

VarTD is the variation in taxonomic distances between every pair of species recorded in an assemblage around the value of AvTD (Clarke \& Warwick, 2001a).

$$
\Lambda^{+}=\left[\sum \sum_{i<j}\left(\omega_{i f}-\Delta^{+}\right)^{2}\right] /[S(S-1) / 2]
$$

$\omega$ is the branch length between species pairs, $S$ is the number of observed species in the sample and $\Delta^{+}$is taxonomic distinctness as defined previously.

VarTD would be relatively large for a sample that contained clusters of species belonging to the same genus (contributing short path-lengths), but where the different clusters themselves are not necessarily closely related (contributing long path-lengths). Importantly, the VarTD is independent of the average taxonomic distinctness AvTD, therefore it measures a different aspect of the taxonomic structure. 
Table 2.1 Branch weighting values for calculating taxonomic distinctness indices. The branches are weighted by the proportion of species using a higher taxonomic classification (for example all the species using a sub order within their taxonomic tree) compared to the total number of species recorded in New Zealand waters.

\begin{tabular}{|l|l|l|l|}
\hline Taxon & Weight & $\begin{array}{l}\text { Branch Length } \\
\text { Species - Class }\end{array}$ & $\begin{array}{l}\text { Branch Length } \\
\text { Genera - Class }\end{array}$ \\
\hline Species & 1.00 & 17.21 & N/A \\
\hline Subgenus & 0.18 & 20.31 & N/A \\
\hline Genus & 1.00 & 37.52 & 21.60 \\
\hline Subfamily & 0.11 & 39.42 & 23.97 \\
\hline Family & 1.00 & 56.63 & 45.57 \\
\hline Suborder & 0.45 & 64.37 & 55.29 \\
\hline Order & 1.00 & 81.58 & 76.89 \\
\hline Subclass & 0.07 & 82.79 & 78.40 \\
\hline Class & 1.00 & 100 & 100 \\
\hline
\end{tabular}

These measures of phylogenetic relatedness of species within a sample in relation to the species present in the regional species list are particularly suitable to presence only data sets with varying historical sampling effort as long as the sampling unit has more than ten different species within it (Rowden et al., 2004). AvTD and VarTD have been found to be correlated in some studies of bryozoans and molluscs (Warwick \& Turk, 2002; Rowden et al., 2004). However, studies on nematodes and demersal fish have found AvTD and VarTD to be independent of each other (Clarke \& Warwick, 2001a; Tolimieri \& Anderson, 2010). If a significant correlation is found between the patterns produced from the species and genera data, then the genera data can act as a surrogate for the species data for comparing the validity of the bioregions and the EEZ wide study.

Initial spatial patterns of biodiversity were explored using the statistical software package PRIMER version 6.1.13 with the PERMANOVA+ add-on 
version 1.0.3 (Clarke \& Gorley, 2006 ). The data were reduced to 'presence only' records and then subjected to multivariate analysis using the Bray Curtis similarity measure to create a resemblance matrix. The resemblance matrices for the port species level data and the general level data were then compared using the RELATE function in PRIMER using 9999 permutations to predict the Spearmans Rank coefficient; this gives the probability of the genera and species data showing significantly similar or different biodiversity patterns. Non-metric Multidimensional Scaling ordination (MDS) plots were used to graphically represent the relationships between the different port sponge species/genera richness and the SIMPROF function was used to elucidate any significant clustering of similar port species richness by carrying out 9999 simulation permutations of each resemblance matrix. SIMPROF is relevant as the port data are each single sampling sessions and therefore they are not a priori divided into groups with replicates.

\subsubsection{SPONGE BIODIVERSITY PATtERnS AROUND THE NEW ZEALAND EEZ}

All data were reduced to presence/apparent absence records (recording the presence at a location rather than true presence/absence data). This is because no areas in New Zealand have been extensively surveyed enough to say that any of the species are definitely absent and may in fact be a 'non-detection' rather than a true absence. It is important to take into consideration that these presence/apparent absence records will most likely result in under-estimates of species distributions and richness. All presence records within a spatial unit were used in each analysis as long as the total records had ten genera or more. Although the taxonomic distinctness indices are largely independent of sample size, there can be a small bias in the results leading to an underestimate of their values with very small species lists (Clarke \& Warwick, 1998; Pienkowski et al., 1998; Clarke \& Warwick, 2001b). Therefore only data sets with ten or more O.T.U.'s will be used in a similar manner to Rowden et al. (2004). If there were 
less than ten genera that unit was not included in the analysis, however, it could contribute to the total in a larger unit. The spatial units were the $0.5^{\circ}$ by $0.5^{\circ}$, latitude and longitude grid squares, bioregions according to Shears et al. (2008) and the DOC bioregions. The grid squares will change area slightly from northern to southern New Zealand due to the degrees of longitude getting closer together as they get closer to the south pole. It was decided that for this study of presence data it would not make a significant difference to the patterns shown by the sponge biodiversity as other factors such as sampling effort, bias towards collecting a certain group of species and the temporal variation of sampling trips would all be influencing the species detection in an area. The data were further stratified into $5 \mathrm{~m}$ depth categories for initial processing. AvTD and VarTD are known to be less influenced by sampling intensity therefore they were used as biodiversity indices.

AvTD and VarTD were calculated for each of the grid squares stratified by depth. Based on initial observations of the data, AvTD and VarTD vary in a nonlinear way across New Zealand, i.e. there are hotspots within regions independent of latitude or longitude. Therefore a modeling procedure was chosen in order to account for the non-linear distribution of the data. As observations of AvTD and VarTD were for specific locations and at specific depths a modeling procedure was chosen that was capable of identifying the differences in values caused specifically by depth, or location independent form each other. Generalised additive models (GAMs) were fitted in R (version 2.11.0 using the mgcv package) (Hastie \& Tibshirani, 1990; Wood, 2006b). In order to investigate how AvTD and VarTD varied with latitude, longitude and depth, all possible models incorporating single terms and all possible interactions were analysed ( $\mathrm{n}=63$ for AvTD and VarTD).

Modeling latitude, longitude and depth allowed the variation in AvTD and VarTD to be mapped as a function of location and also of depth, essentially allowing the possibility of modeling the 3 dimensional variations of these parameters. These variables were included as factors in the model fit and also 
as interaction terms as it was expected that the variation due to latitude, longitude and depth to vary depending on one or more of the other predictor variables. For example, including the interaction term latitude*depth allows the effects of latitude to vary between different depth regions, and subsequently the effects of depth to vary between different latitudes. This similarly applies to the other interaction terms, latitude*longitude and longitude*depth, which allows a more accurate description of how taxonomic distinctness varies around New Zealand coastal waters.

For these analyses all $0.5^{\circ}$ Latitude by $0.5^{\circ}$ Longitude areas with at least ten sponge genera recorded within them in at least one depth group less than $150 \mathrm{~m}$ were used (See Fig. 2.4 red rectangles). The depth range was reduced to $150 \mathrm{~m}$ after preliminary analysis because the majority of the $>150 \mathrm{~m}$ species records (with more than 10 genera present) were off N.E. New Zealand and therefore were not representative for patterns for New Zealand coastal waters.

GAMs were fitted according to the following equation:

$\gamma_{i}=\beta_{0}+I_{1, k} \cdot f_{1}\left(D_{i}\right)+I_{2, k} \cdot f_{2}\left(\theta_{i}\right)+I_{3, k} \cdot f_{3}\left(\theta_{i}\right)+I_{4, k} \cdot f_{12}\left(D_{i} \times \theta_{i}\right) I_{5, k} \cdot f_{13}\left(D_{i} \times \phi_{i}\right)+I_{6, k} \cdot f_{23}\left(\theta_{i} \times \phi_{i}\right)+\varepsilon_{i}$

where $\gamma_{i}$ is either AvTD or VarTD, $\beta_{0}$ is the intercept parameter $D_{i}$ is the depth, is the $\theta_{i}$ latitude and $\phi_{i}$ is the longitude. $I_{n, k}$. is a vector of binary values with values depending on which terms are retained in the model and equals 1 when the $\mathrm{n}^{\text {th }}$ term is included in the model, and zero when it is excluded. $f_{1,2,3}$ are thin plate regression splines of the variables, Depth, Latitude and Longitude. The best function $f_{i}$ is selected based on a quadratically penalised scoring system (Wood, 2006b, a). 


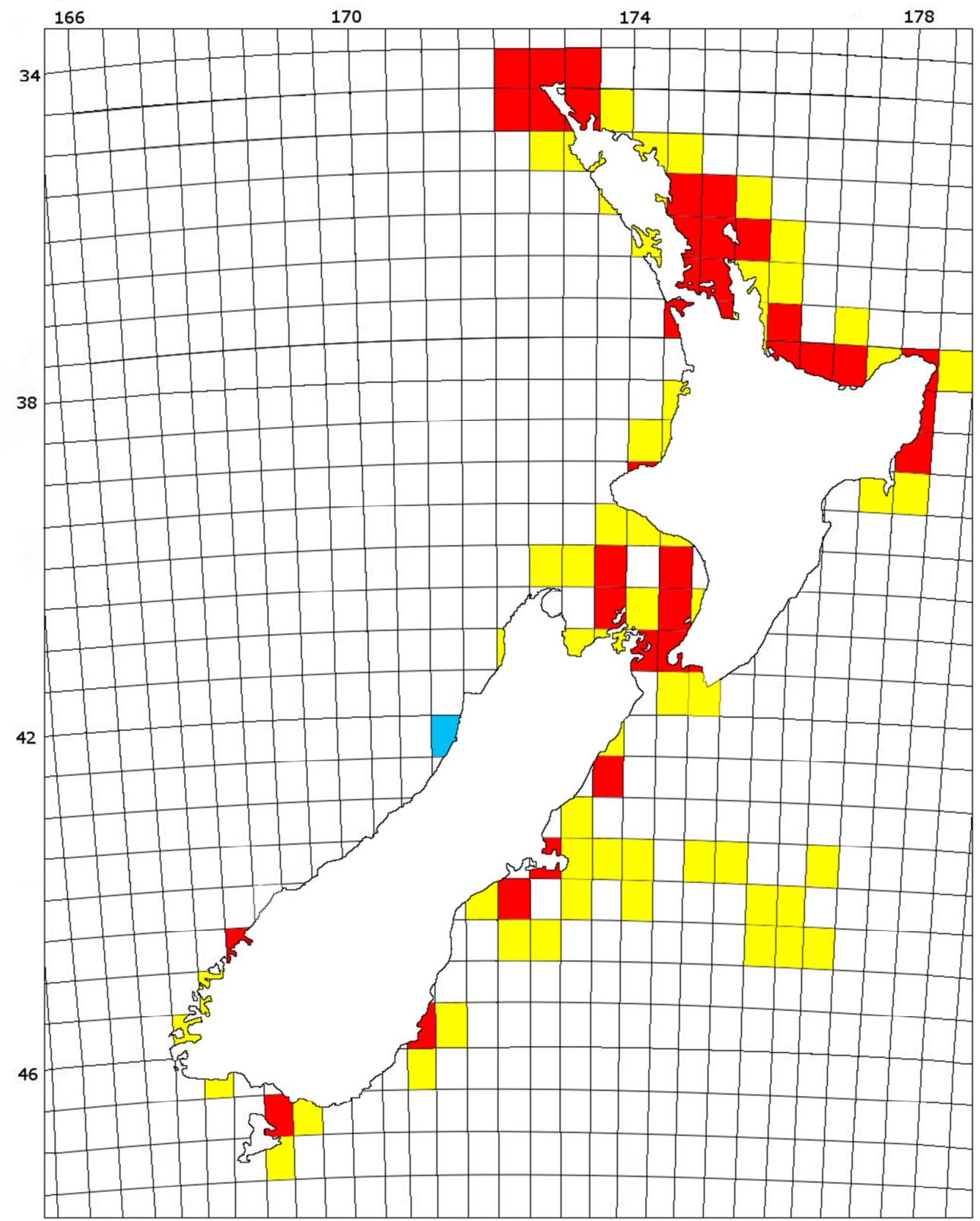

Fig. 2.4 Porifera records around mainland New Zealand. Red indicates $0.5^{\circ}$ Latitude by $0.5^{\circ}$ Longitude areas with at least ten sponge genera recorded within them in at least one depth category (some of the squares have multiple records across a range of depths). Yellow indicates that there were one to nine records within a single depth category. These yellow records have been used to calculate the average values for the bioregions. The blue rectangle of the South Island West coast was not used towards the bioregion analysis as there were only two different genera recorded from that area and there were not enough close records in order to use it in this analysis. 
Each alternative model fit of AvTD/VarTD to depth, latitude and longitude values around New Zealand is scored based on its likelihood, but is penalised for higher order terms reducing the overall score for more complicated models. This in essence means that lower order terms (creating simpler models with fewer factors) are preferred in the smoothing selection procedure. Because latitude, longitude and depth were recorded on different scales, $f_{1,2}, f_{1,3} f_{2,3}$ were tensor product smooths of thin plate regression splines (which form an arc between the factors) for the various interaction terms. $\varepsilon_{i}$ was the residual error term which was assumed to be normally distributed with zero mean and a common variance (Tolimieri \& Anderson, 2010).

Akaike information criterion (AIC) values for the various model fits were extracted and models were ranked according to AIC. AIC is a measure of the goodness of fit of an estimated statistical model grounded in the concept of entropy to test between models (Akaike, 1987). In effect, it offers a relative measure of the information lost when a given model is used to describe reality and can be said to describe the tradeoff between the accuracy and complexity of the model (Tolimieri \& Anderson, 2010). Differences of less than two in AIC values indicates that the models are indistinguishable and the model explaining more of the variance provides the better model (Burnham \& Anderson, 2002).

After preliminary analysis exploring all records from 0-200m depth range, the records which were $50 \mathrm{~m}$ or shallower were selected as these records were more evenly dispersed around New Zealand and not as Northern New Zealand heavy. Then the possible models for using latitude and longitude as factors were calculated. The equation used for these models was as above however all terms involving Depth $\left(D_{i}\right)$ were dropped. The results and summary statistics for the highest ranked models based on AIC for depth as a factor and also the shallower model $(<50 \mathrm{~m})$ are presented in the results. 


\subsubsection{SPONGE BIODIVERSITY WITHIN BIOREGIONS}

As the presence only data is patchily distributed around New Zealand (see Fig. 2.4) this is a first attempt to determine how sponge diversity is distributed around New Zealand in relation to the bioregions shown in Fig. 2.2. Although the bioregion boundaries are artificial they are based on the assemblage data from other phyla including algae, invertebrate and fish data. All sponge records within the boundaries of a bioregion (0-200m depth) were included as long as the total number of records of different genera within the spatial unit 0.5 lat/long square, bioregion Shears, bioregion DOC, Biogeographic Province) reached ten or higher. The data were reduced to 'presence only' records and then subjected to multivariate analysis using the Bray Curtis similarity measure to create a resemblance matrix. MDS plots were used to visualise patterns in the presence biodiversity data in relation to depth, latitude and longitude. One-way ANOSIM was used to calculate the significance of the factors individually and values of AvTD and VarTD were compared between significant bioregions. 


\subsection{RESULTS}

\subsubsection{SPECIES AND GENERA LEVEL ANALYSIS}

The number of species per port site ranged between 25 and 46 with a mean of $32.8 \pm 7.21$ S.D. These species were spread across 12 to 23 genera with a mean of $17.3 \pm 3.34$ S.D. The linear regression for the species versus genera data was $y+0.38 x+4.91$ with a $R^{2}=0.67$, which was significant $(P<0.05)$ (Fig. 2.5).

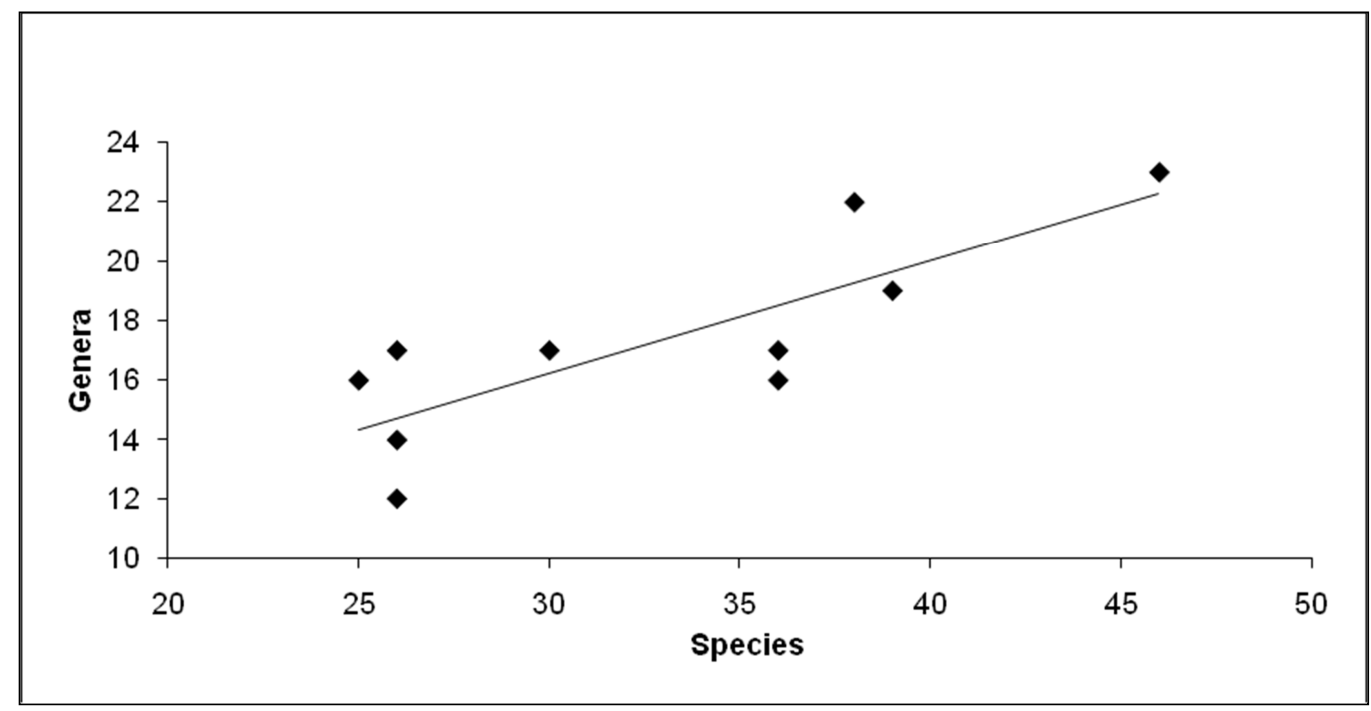

Fig. 2.5 The relationship between genera and species diversity. At each of the port sites with more than 10 genera present the number of species and genera present were compared. The linear regression equation is $y=0.38 x+4.91 R^{2}=0.67$.

A significant correlation was found between the relationship of AvTD values for the genera and species level data $\left(\mathrm{R}^{2}=0.81\right)$. Genera AvTD values explained $81.1 \%$ of the variation in AvTD species data (FIG. 2.6). Therefore AvTD for genera can be used as a surrogate biodiversity measure for species AvTD. It is important to note that the Lyttelton port data point was an outlier compared with the other nine port sites therefore it was excluded from the linear regression analysis. If the Lyttelton data point was included then $R^{2}=0.47$ and only $46.8 \%$ of the variation was explained. Reasons for why Lyttelton does not fit the pattern of the other sites will be addressed in the discussion. 


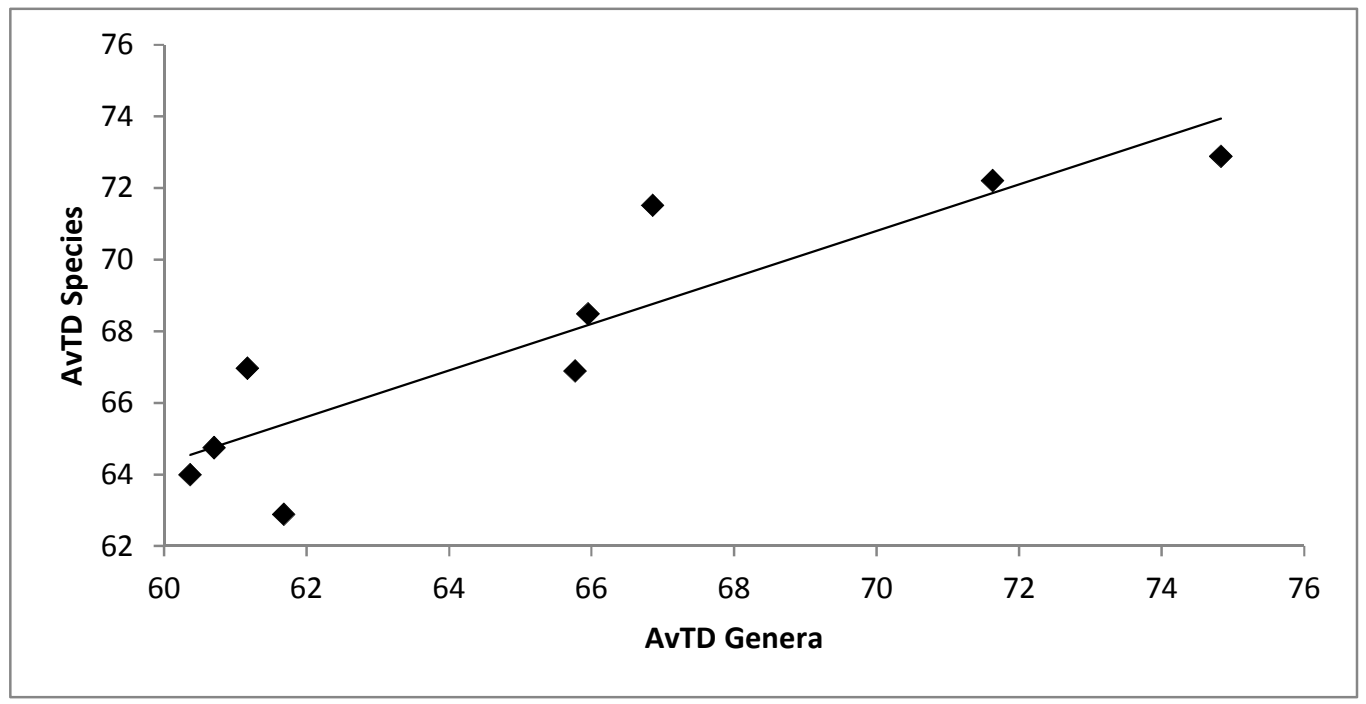

Fig. 2.6 The relationship between AvTD for genera and species port data. The equation for the linear regression line is $y=0.65 x+25.31$ and $R^{2}=0.81$. Therefore the genera AvTD explains $81.1 \%$ of the variation in AvTD species data.

For VarTD there was no correlation between the species and general level data, although there was a tendency for VarTD to be lower for the genera data versus the species data. VarTD correlates with AvTD, however, the relationship is different for the species data (AvTD increases, VarTD decreases) and genera data (AvTD increases, VarTD increases) (Fig. 2.7). The species VarTD explained approximately $65 \%$ of the variation in AvTD and vice versa. The genera VarTD only explained approximately 55\% of the variation in AvTD. Therefore there was a $10 \%$ drop in the amount of variance explained by the genera AvTD and VarTD in comparison with the species AvTD and VarTD values. 


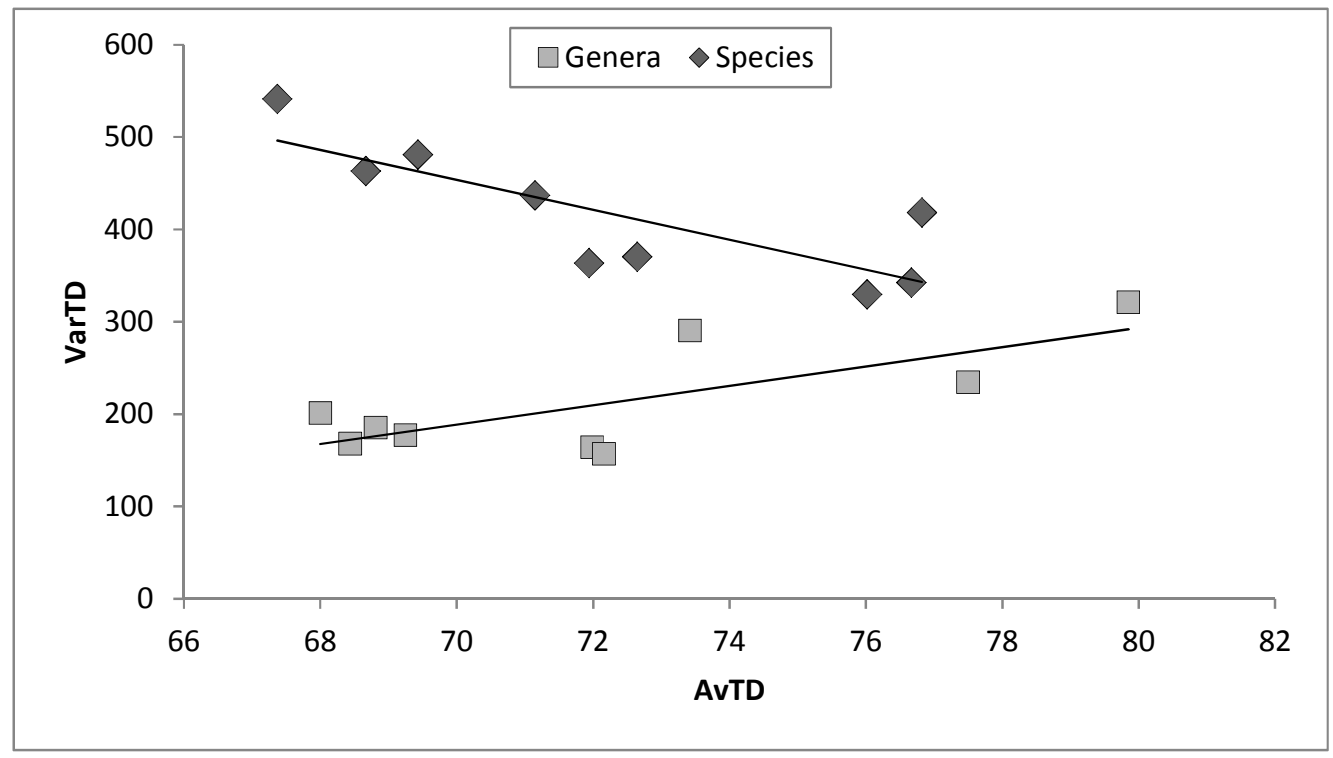

Fig. 2.7 The relationship between VarTD and AvTD for genera and species data. This plot shows that for species level data (Diamond markers) AvTD increases VarTD tends to decrease. The equation for the linear regression line is: $y=-16.20 x+1587.5$ and $R^{2}=0.65$. When the dataset was reduced to genera level (Square markers) AvTD is positively correlated with VarTD. The equation for the linear regression line is $y=10.46 x-543.4$ and $R^{2}=0.55$.

When the Bray Curtis resemblance matrices were compared, the Spearmans Rank correlation coefficient was also significant Rho $=0.74(\mathrm{P}<0.01 \% ; 9999$ Permutations), which means that the matrices created by the species and genera data are significantly correlated and therefore genera level data were used in the rest of the biodiversity analyses. 


\subsubsection{BIODIVERSITY IN THE EEZ}

4735 Porifera records, encompassing 805 sponge species/operational taxonomic units representing 215 genera, 99 families and 20 orders and three classes, were examined in this study in the New Zealand EEZ down to $200 \mathrm{~m}$. The most specious families were the Chalinidae (mainly species from the genus Haliclona - 52spp.), Ancorinidae (mainly from the genus's Ancorina and Stelletta - 51spp.), and Halichondriidae (main contributors were Halichondria, Clioclapta and Hymeniacidon genus's - 50spp.). The Poecilosclerida were by far the most specious order with 232 species although the species were spread across many families rather than clustering within one or two families. The Haplosclerida was the second largest order, containing 143 species including the Chalinidae and Callyspongidae families, while the Halichondrida order had 94 species, including the Halichondriidae and Axinellidae families. Across all the classes there were 753 species of Demosponges, 44 Calcareous species and 8 Hexactinellids were recorded.

The twenty most common genera across the region had wide depth, latitudinal and longitudinal ranges (Table 2.2). Fifty of the genera were only recorded once (in one spatial unit) and forty-three were recorded in only two units, while Callyspongia was the most frequently recorded genus, and was present in 145 different spatial units. 
Table 2.2 The twenty commonest genera recorded from $0-200 \mathrm{~m}$ within the New Zealand EEZ. Each presence record for a genus represents at least one record within the $0.5^{\circ}$ by $0.5^{\circ}$ latitude/longitude unit within a depth group. The depth, latitude and longitude range are also shown.

\begin{tabular}{|c|c|c|c|c|}
\hline Genera & Presence Records & $\begin{array}{c}\text { Depth range } \\
\text { (m) }\end{array}$ & Latitude range & Longitude range \\
\hline Callyspongia & 145 & $0-200$ & $34-52.5$ & $166-183.5$ \\
\hline Tethya & 98 & $0-140$ & $34-47$ & $167.5-183.5$ \\
\hline Latrunculia & 91 & $0-200$ & $34-50.5$ & $166.5-183.5$ \\
\hline Haliclona & 90 & $0-200$ & $34-52.5$ & $166-183.5$ \\
\hline Stelletta & 84 & $0-200$ & $34-51$ & $170-183.5$ \\
\hline Ancorina & 72 & $0-200$ & $34-43.5$ & $172-177$ \\
\hline Iophon & 72 & $0-200$ & $34-47.5$ & $167-183.5$ \\
\hline Crella & 70 & $0-200$ & $34-48$ & $167-183.5$ \\
\hline Tedania & 68 & $0-200$ & $34-50.5$ & $166-183.5$ \\
\hline Halichondria & 62 & $0-200$ & $34-51$ & $166-183.5$ \\
\hline Polymastia & 61 & $0-100$ & $34-51$ & $166-178$ \\
\hline Clathria & 60 & $0-200$ & $34-52.5$ & $167-178$ \\
\hline Darwinella & 57 & $0-90$ & $34-47$ & $167-183.5$ \\
\hline Mycale & 57 & $0-200$ & $34-46.5$ & $167.5-178$ \\
\hline Dactylia & 51 & $0-140$ & $34-46$ & $170.5-184.5$ \\
\hline Hymendacidon & 50 & $0-120$ & $34-52.5$ & $166-179$ \\
\hline Aaptos & 49 & $0-70$ & $36-41$ & $172.5-179$ \\
\hline Cliona & 47 & $0-100$ & 34.46 .5 & $168-183.5$ \\
\hline Raspailia & 47 & $0-140$ & $34-45.5$ & $167-183.5$ \\
\hline Suberites & 47 & $0-140$ & $34-51$ & $166-178.5$ \\
\hline
\end{tabular}

One hundred and sixty four of the $0.5^{\circ}$ latitude by $0.5^{\circ}$ longitude spatial units only had one genus recorded within them and 76 had only two genera within their boundaries. In total there were 367 spatial units out of 451 with less than ten genera present that could therefore not be included within the further 
spatial unit analyses, however, they were incorporated into the later bioregion analyses. The remainder of the analyses in this section used the 84 spatial units with at least 10 genera records within them.

The MDS plot of the sponge data for the spatial units shows that there is a clear shift in the sponge assemblage from the shallow to deeper waters (Fig. 2.8), The stress level on the plot was quite high at 0.28 , however, these patterns are supported up by the 1-way ANOSIMs for the factors Depth $(\mathrm{Rho}=0.32$, $\mathrm{P}<0.01 \%)$, Latitude $(\mathrm{Rho}=0.23, \mathrm{P}<0.02 \%$; and Longitude $\mathrm{Rho}=0.37, \mathrm{P}<0.01 \%)$ ) all with 9999 permutations and no permuted statistics greater than Rho $=0$.
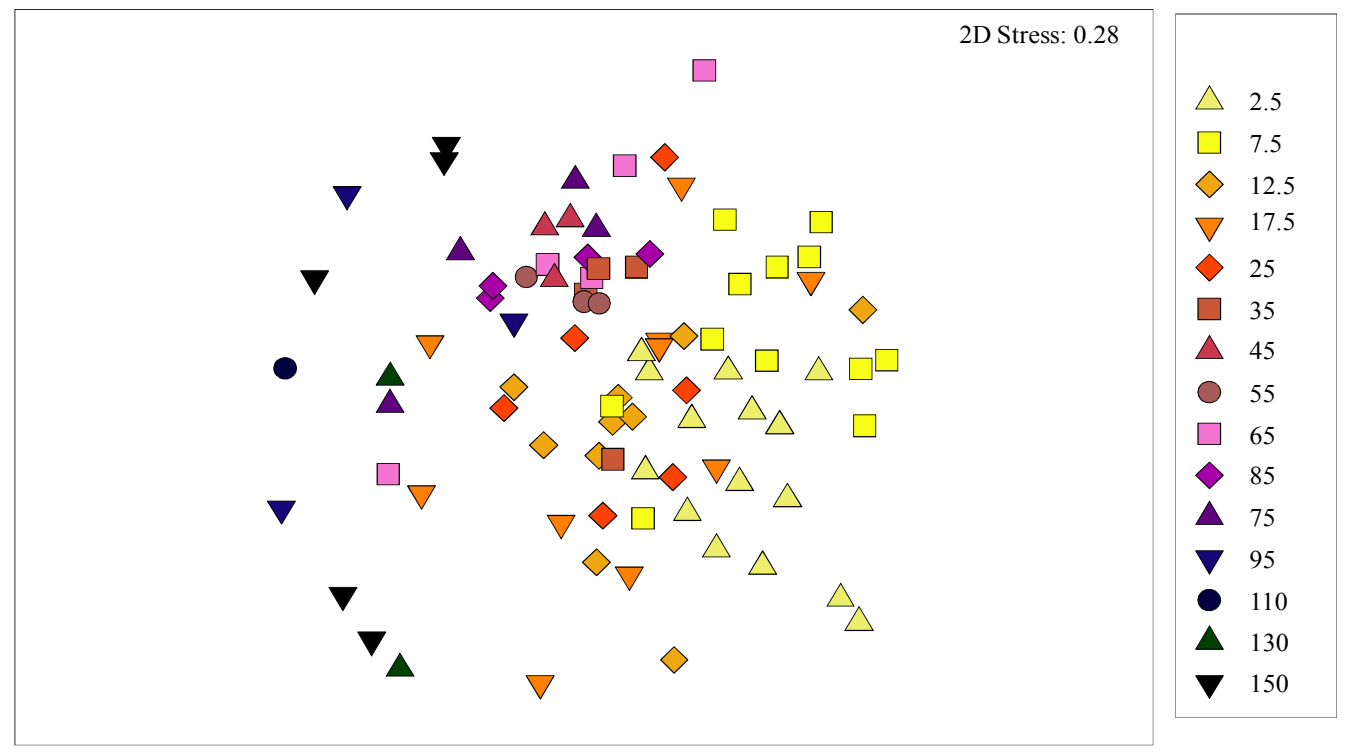

Fig. 2.8 MDS plot showing sponge assemblage changes with depth. Each data point within this MDS plot represents a $0.5^{\circ}$ lat/long grid square which has more than ten genera present within that depth category. It was created from a Bray Curtis Similarity matrix using presence only data. The legend at the side lists the mean depth in $(m)$ for each data point.

The AvTD and VarTD values change with depth, latitude and longitude (Fig. 2.911). Most sites fell within the $95 \%$ probability funnel for AvTD, which means they are not significantly different from the average values for New Zealand waters, however, many of the northern (higher latitude) sites had lower than expected values of AvTD meaning that they have less diversity than would be expected in New Zealand waters for the number of genera recorded. The values 
of AvTD and VarTD of the shallowest sites $(0-30 \mathrm{~m})$ had the widest range with the lowest and highest values recorded for New Zealand, however, all the sites above the mean predicted for AvTD were shallower than $20 \mathrm{~m}$ and the deepest records tended to have lower values of AvTD (Fig. 2.9). For VarTD there was no clear pattern with depth and almost all of the sites fell within the 95\% probability funnel and therefore were not significantly different from each other in relation to depth although there was a trend for the deepest sites to have higher values of VarTD.
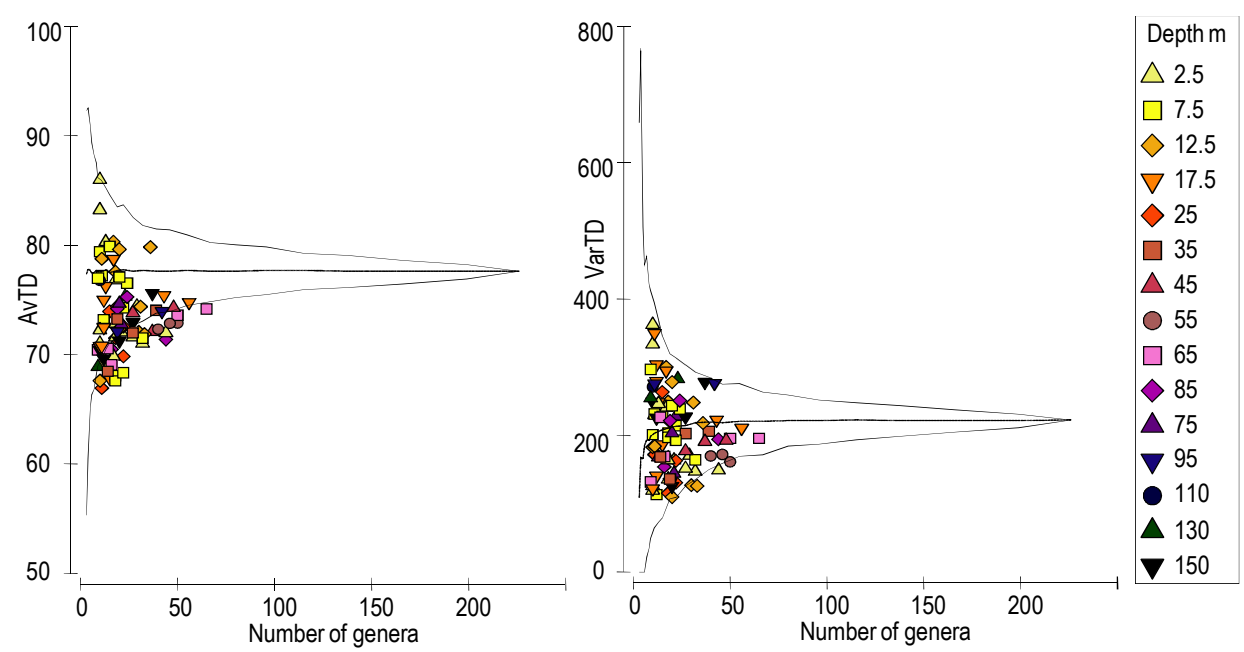

Fig. 2.9 AvTD and VarTD variability with depth changes in New Zealand waters. The central line on both graphs indicates the mean AVTD (left) and VarTD (right), respectively, for the master list of sponge genera recorded from New Zealand. The funnels constitute the $95 \%$ limits of the simulated AvTD (a) and VarTD (b) values. The points on each graph are plotted against the number of genera present at each site and are colour coded for depth according to the legend in the top right corner which applies to both graphs. 

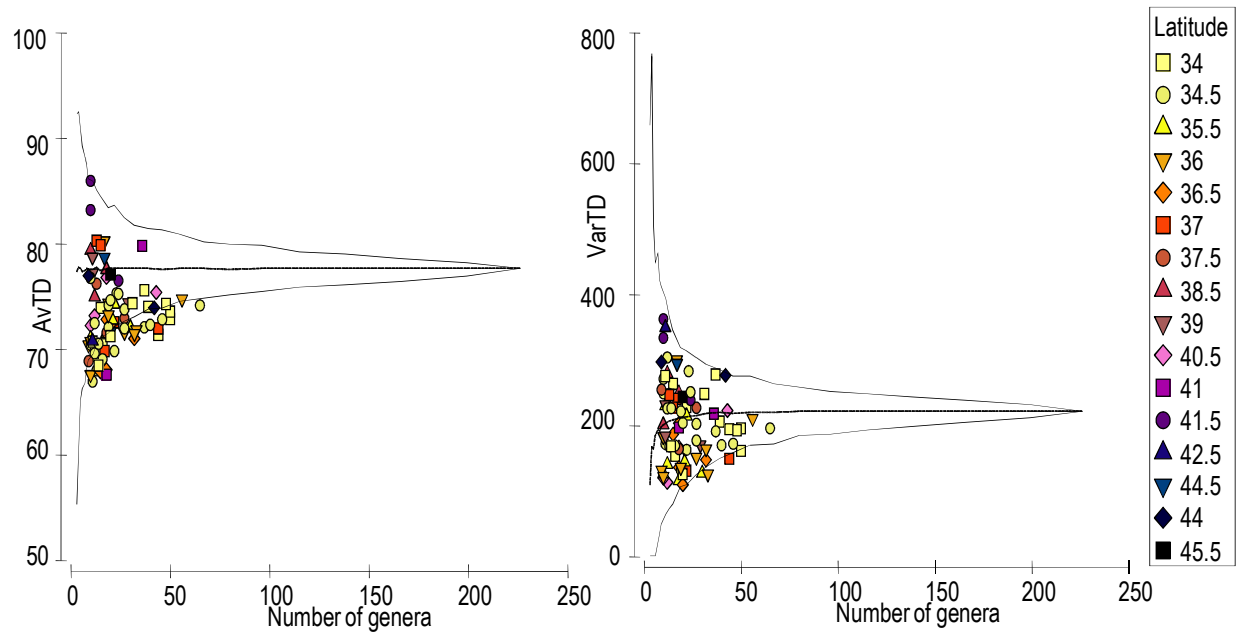

Fig. 2.10 AvTD and VarTD variability with latitude changes in New Zealand waters. The central line on both graphs indicates the mean AvTD (left) and VarTD (right) respectively for the master list of sponge genera recorded from New Zealand. The funnels constitute the $95 \%$ limits of the simulated AvTD (a) and VarTD (b) values. The points on each graph are plotted against the number of genera present at each site and are colour coded for latitude according to the legend in the top right corner which applies to both graphs.

For latitude, the southernmost sites had higher values for AvTD and VarTD and the northernmost sites had some of the lowest values of AvTD and VarTD (Fig. 2.10). Longitude tended to show aggregations of sites but no clear transition from east to west (Fig. 2.11). The spatial units from the shallow $(<10 \mathrm{~m})$ Wellington South coast had the highest values of AvTD and VarTD and therefore even though the species richness was not the highest, the O.T.U.'s recorded are from a wider range of families and orders then other areas surveyed. These sites were sampled over time for chapters 3 and 4 due to the high levels of AvTD and VarTD and reasons for the high values will be explored further in those chapters. 

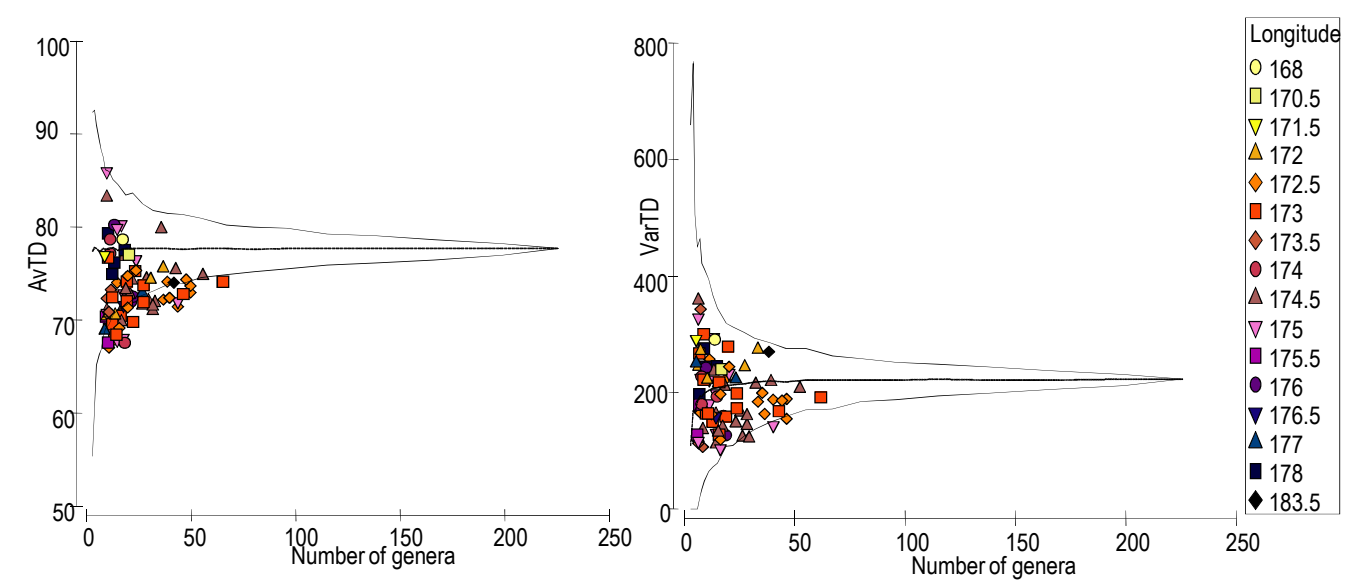

Fig. 2.11 AvTD and VarTD variability with longitude changes in New Zealand waters. The central line on both graphs indicates the mean AvTD (left) and VarTD (right) respectively for the master list of sponge genera recorded from New Zealand. The funnels constitute the $95 \%$ limits of the simulated AVTD (a) and VarTD (b) values. The points on each graph are plotted against the number of genera present at each site and are colour coded for longitude according to the legend in the top right corner which applies to both graphs.

The GAM model that explained the highest amount of the variation in AvTD $<150 \mathrm{~m}$ used the factors Depth, Latitude ( $\mathrm{F}=7.73 \mathrm{p}<0.01)$, and the interactions Latitude*Longitude $(\mathrm{F}=2.15 \mathrm{p}<0.05)$, Depth*Latitude and Depth*Longitude $(\mathrm{F}=5.15, \mathrm{p}<0.05)$. This model explained $54.6 \%$ of the variation in AvTD $\left(\mathrm{r}^{2}=\right.$ $0.41, n=81$ ) therefore just over $50 \%$ of the variation in AvTD can be explained location and depth and just under $50 \%$ is due to stochastic processes or other factors. The AIC value for this model (AIC= 420.61) was marginally lower than one of the other models (AIC $=420.53)$. Model selection procedures based on AIC select the most likely model, but are penalised for the number of parameters. The model identified explained more of the variability in AvTD (54.6\% compared to $51.3 \%$ ), but had a lower AIC value because this model was more complex (two factors and three interaction terms, compared to one factor and three interaction terms) and so was penalised when calculating AIC. However, competing model AIC values differed by $<0.1$ and as such models can be considered equivalent. As such the chosen model was selected as it explained 
more of the variability in the dataset compared to the competing model (Commenges et al., 2008). (See Appendix 2.7 for all of the models tested). The intercept (âo) was $73.41 \pm 0.32$ S.E., which indicates that on average, genera were related between the levels of suborder and order so a random sample is likely to contain genera from different orders and suborders and have diverse evolutionary lineages (Table 2.1).

The best model for VarTD $(<150 \mathrm{~m})$ according to AIC (854.34) used the factors Depth $(\mathrm{F}=3.29 \mathrm{p}>0.05)$, Latitude $(\mathrm{F}=3.22, \mathrm{p}<0.01)$, Longitude $(\mathrm{F}=8.42 \mathrm{p}>0.01)$ and the interactions between Depth*Latitude, Depth*Longitude, and Latitude*Longitude $(\mathrm{F}=2.82, \mathrm{p}<0.05)$. This explained $57.3 \%$ of the variation in $\operatorname{VarTD}\left(\mathrm{r}^{2}=0.47, \mathrm{n}=81\right)$ and approximately $40 \%$ is due to stochastic processes or other factors.

To further investigate the coastal water patterns related to latitude and longitude, the records from $50 \mathrm{~m}$ or shallower were reexamined ignoring depth as a factor (so all the $0-50 \mathrm{~m}$ presence records were grouped together). The best model for AvTD $(<50 \mathrm{~m})$ according to AIC (327.21) used the factors Latitude, Longitude and interactions between Latitude*Longitude. This model explained $42.6 \%$ of the variation in AvTD $\left(r^{2}=0.32, n=60\right)$ therefore approximately $60 \%$ of the variation was due to stochastic processes or other factors which will be taken into account in the discussion. The intercept (â) was $73.79 \pm 0.45$ S.E., which indicates that on average, genera were related between the levels of suborder and order (Table 2.1).

The best model for VarTD $(<50 \mathrm{~m})$ according to AIC (652.68) used the factors Latitude and the interactions between Latitude*Longitude. This explained $42.8 \%$ of the variation in $\operatorname{VarTD}\left(\mathrm{r}^{2}=0.33, \mathrm{n}=60\right)$. 
In order to visualise the models effectively, the fitted values for AvTD and VarTD for the full data range and the shallower 0-50 $\mathrm{m}$ range have been overlaid onto maps of New Zealand (Fig. 2.12). These show that when the whole data set is used there is little variation in AvTD and hotspots of VarTD around the south of the South Island, Kaikoura and Wellington. In the shallow data set AvTD and VarTD show similar patterns of biodiversity with the hotspots again in the south of the South Island, Kaikoura and Wellington. 

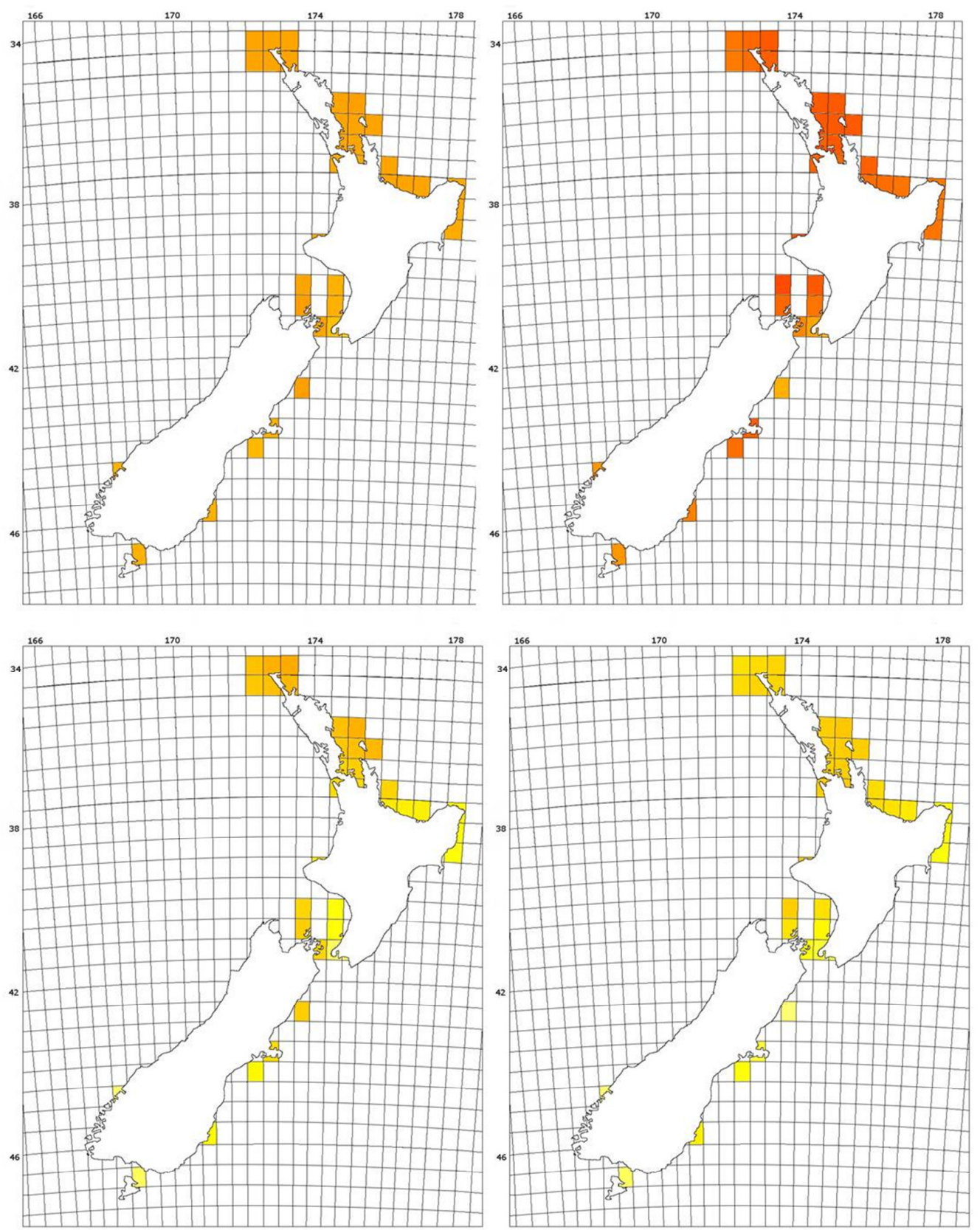

Fig. 2.12 AVTD and VarTD models related to the New Zealand coastline. The maps on the left show the variation in AvTD around New Zealand and the maps on the right show the variation in VarTD. The top two maps relate to the New Zealand dataset to $150 \mathrm{~m}$ and the bottom two maps show the reduced data set from $0-50 \mathrm{~m}$. Lighter (yellow) colours depict higher values and darker (red) depict lower values. 


\subsubsection{SPONGE BIOREGIONS}

When the $0.5^{\circ}$ by $0.5^{\circ}$ latitude and longitude records of sponge genera presence data (with more than ten records) were analysed to see if they correlated with the fourteen DOC bioregions (based on $0-50 \mathrm{~m}$ ) or the bioregions suggested by Shears et al. (2008) using SIMPROF, there were no significant clusters within either bioregion scheme. However, when all the presence records of species listed within a bioregion $(0-50 \mathrm{~m}$ records) for the DOC bioregions or those designated by Shears et al. (2008) were compared with the other bioregion species lists there is a relationship in the similarity between the bioregions and the distances they are apart around the coastline of New Zealand (Fig. 2.13 and Fig. 2.14). It is important to consider that these data tend to be concentrated around point locations, for example, Wellington in the Cook Strait rather than spatially evenly distributed data points. The lack of data from the west coast of the South Island, which includes the Buller bioregion (four genera) and Westland (no genera) meant they were not included in any of the analyses. This was also the case for Snares Island (no genera) and the Kermadecs (two genera). There were limited data available from Campbell Island (eight genera), the Bounty Islands (eight genera) and the Antipodes (seven genera) therefore they were not included in the Shears et al. (2008) bioregions but were included as part of the Sub Antarctic bioregion for DOC. 


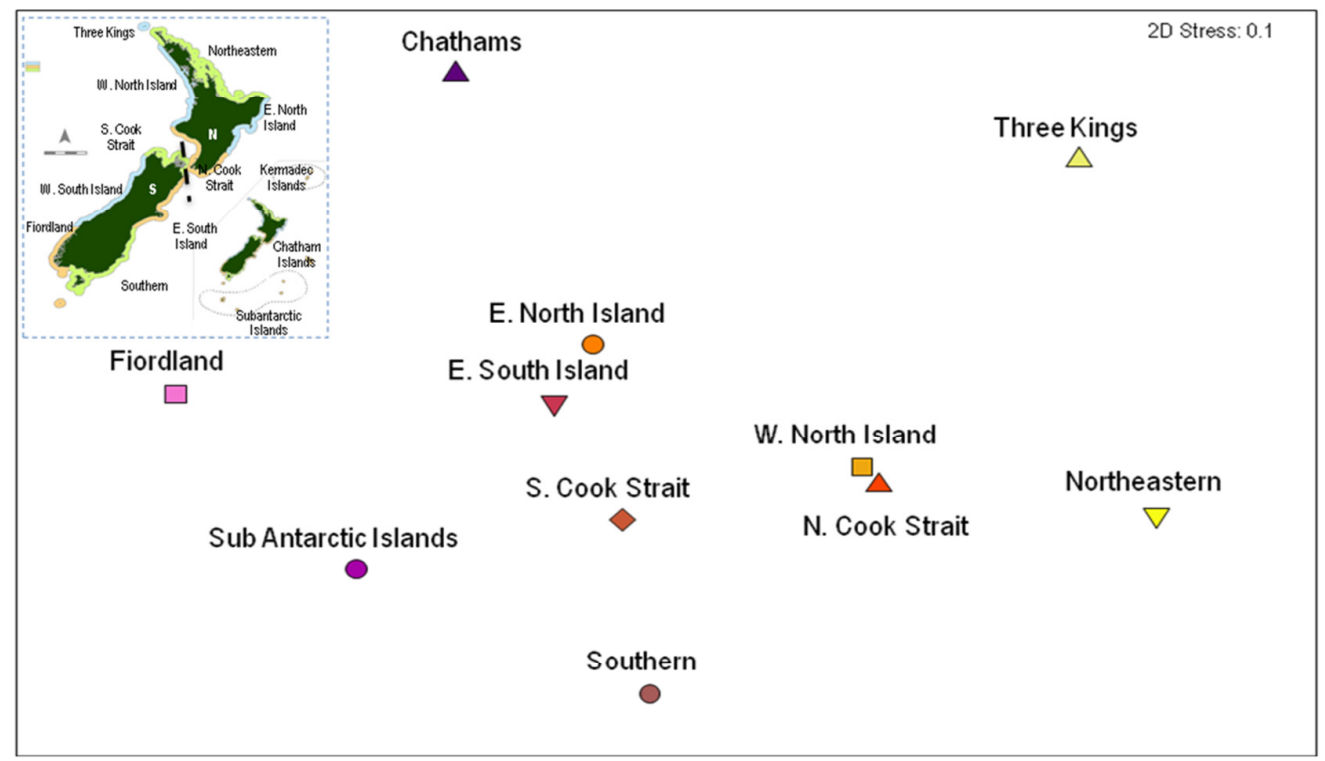

Fig. 2.13 MDS plot of DOC bioregions $(0-50 \mathrm{~m})$. MDS plot created from a Bray Curtis similarity matrix depicting the sponge assemblages from each of the bioregions with more than ten genera present at $0-50 \mathrm{~m}$ depth. Inset is a map of the DOC bioregions.

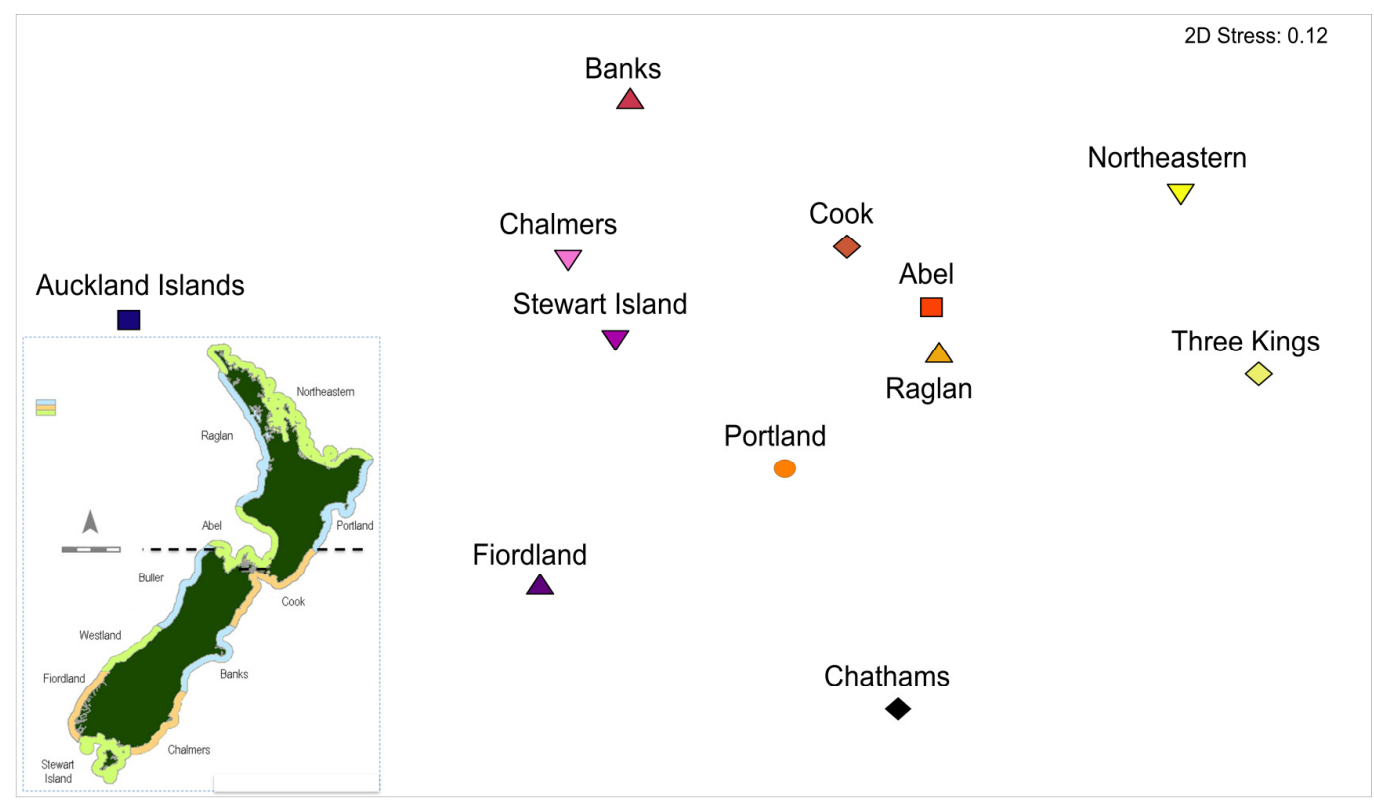

Fig. 2.14 MDS plot of Shears (2008) bioregions (0-50m). MDS plot created from a Bray Curtis similarity matrix (presence/absence transformed) depicting the sponge assemblages from each of the bioregions with more than ten genera present at $0-50 \mathrm{~m}$ depth. Inset is a map of the Shears et al., (2008) bioregions. 
The values of AvTD did not vary significantly with depth for any of the DOC bioregions. VarTD did vary between the $0-200 \mathrm{~m}$ and $0-50 \mathrm{~m}$ bioregions for the Three Kings Islands and the Chatham Islands with higher values for the wider depth range. AvTD remained relatively consistent between the bioregions however, VarTD was higher for the North Cook Strait, Fiordland, and Southern and particularly low for the Sub Antarctic Islands, Chatham Rise and Western North Island. The Three Kings Islands were high for the wider depth range and low for the shallow depth range. All of the values of AvTD fell within the 95\% probability limits for New Zealand, however, the Chatham Islands and Three Kings were on the lower limit. All the values of VarTD fell within the $95 \%$ probability limits for all the bioregions with ten or more genera present.

The values of AvTD did not vary significantly with depth for any of the Shears bioregions. VarTD did vary between the $0-200 \mathrm{~m}$ and $0-50 \mathrm{~m}$ bioregions for the Three Kings Islands. AvTD remained relatively consistent between the bioregions however, VarTD was higher for the North Cook Strait, Fiordland, and Stewart Island and particularly low for Raglan and Banks bioregions.

All of the values of AvTD were within the 95\% probability limits for New Zealand, however, the Chatham Islands, Auckland Islands and Three Kings were at the lower limit. All the values of VarTD were within the 95\% probability limits for all the bioregions with ten or more genera present. 


\subsection{DISCUSSION}

There was a clear shift in the biodiversity of sponge assemblages with depth; and a combination of depth and location was responsible for about $50 \%$ of the variation in the AvTd and VarTD of the sponge assemblages around New Zealand. Overall, 4735 Porifera records encompassing 805 described and undescribed sponge species, within 215 genera, have been recorded in New Zealand coastal waters from $0-200 \mathrm{~m}$ depth. This is an increase from the 732 known sponge species from all depths within the New Zealand EEZ listed in Kelly et al. (2009).

An exhaustive species list would be ideal for a geo-referenced biodiversity inventory; however, due to funding, expertise and timing limitations this is rarely possible. In terrestrial systems it has been found that presence/absence data reflect the geographic variation better than abundance/biomass data, which are more influenced by local-scale environmental conditions (Steinitz et al., 2005). Presence absence/data has also been found to reflect the regional patterns for sponges in the Mediterranean (Voultsiadou, 2009), Indonesia (de Voogd \& Cleary, 2008) and for other marine taxa such as algae, (Goldberg et al., 2006) molluscs (Terlizzi et al., 2009) and fish (Gladstone \& Alexander, 2005). In tropical Australian waters, sponge species and genera data were weakly correlated within locations (alpha diversity) as genera records underestimated the species biodiversity, however, they did correlate at a regional level (gamma diversity) (Hooper et al., 2002). Good indicator taxa need to reflect the speciation of a genus as different species are successful in different local habitats and regional patterns reflect the phylogeographic patterns that are often better represented by genera data. The results of this study suggest that for sponge biodiversity in New Zealand genera level data can be used as a surrogate for species diversity. 
The number of species recorded and the species AvTD are highly correlated with the number of genera present and genera AvTD. This is useful because currently there is only one professional sponge taxonomist in New Zealand and a handful of para-taxonomists who can identify sponges to species level. There are also over a third of known sponge species that are currently undescribed and there are estimated to be over 500 more unknown sponge species (Kelly et al., 2009; Gordon et al., 2010). On a global-scale there are also many habitats that are known to be species rich, but many of the poorly studied phyla, have been under-sampled and remain unidentified (Costello et al., 2010). In fact, out of an estimated 11.3 million known species on earth, only approximately 1.9 million species had been described by 2007 (Chapman, 2009). For marine invertebrates, the extent of taxonomic knowledge, including the number of species described every year, depends on the size of the taxonomic community studying various groups therefore on average worldwide only 40 sponge species are described each year (Bouchet, 2006).

There were no direct correlations between the species and genera data for Variation in Taxonomic Distinctness (VarTD) for the New Zealand port data set. This lack of correlation reflects the unevenness of the distribution of species within the same genus. For example, some of the samples have multiple species within the genera Haliclona and/or Clathria that contribute lots of short pathlengths. However, different Haliclona and Clathria spp. themselves are not necessarily closely-related as Haliclona is in the order Haplosclerida and Clathria is in the order Poecilosclerida, and therefore contribute long pathlengths. This means that VarTD would be higher even though AvTD would be an average value as the short and long path lengths would balance each other out. Lyttelton Harbour had very different values for AvTD and VarTD for the species data compared with the other ports within the survey. Twelve of the twenty-six species for Lyttelton harbor were Haliclona spp. and there were only ten genera present in total, which accounts for the differences as they were not evenly distributed between the genus level and species level AvTD and VarTD results. 
The reasons for the high number of Haliclona spp. at Lyttelton harbour could be because some Haliclona spp. can exhibit a wide range of morphologies and growth strategies and therefore can undertake a wide range of functional roles within a habitat (Elvin, 1976; Kaandorp, 1991; Bell, 2004; Abdo et al., 2008). Haliclona is also one of the cosmopolitan genera that are found worldwide with no distinctive centre of their distribution range (van Soest, 1994). Haliclona is partly split into sub genera but this is far from complete and sub-genera in Haliclona could have more restricted distributions (van Soest, 1994; van Soest et al., 2008). Haliclona is also a specious genus in New Zealand, as out of the species included in the master taxonomic list, 55 of the species $(12.1 \%)$ were from the genus Haliclona (unpub. data). Therefore it is to be expected that there will be lots of Haliclona spp. in comparison to some of the other genera which leads to it being influential in the species level AvTD and VarTD values for New Zealand sites. There will always be exceptions to the general patterns as in the case of Lyttelton Harbour and this is important to take into consideration when assessing biodiversity, potentially by assessing specious genera separately, however, for all the other sites genera data provided a good indicator of the species richness and taxonomic richness in particular in relation to AvTD.

The sponge assemblage composition in New Zealand waters is influenced by depth, latitude, longitude, and the interactions between these three factors. This means that as well as direct correlations with the three factors there was a strong effect from other factors such as the regional species pool of the local assemblages. The influence of regional species pools on local benthic assemblages has been reported on a global scale by Witman et al., (2004). The last Pleistocene Glaciation, which occurred about 2.4 million years ago, has been considered to be an important event in determining present-day assemblages in New Zealand, as this time period also coincided with intense tectonic activity. The presence of glacial ice masses and altered climatic conditions combined with geological uplift created distinct environmental types and may have possibly created refuges that may have shaped local populations for example, 
the endemic black coral in Fiordland and southern Stewart Island (Miller, 1998). The New Zealand region is still active tectonically, with areas of active uplift and subduction and the formation of deep-sea trenches, active seamounts and volcanoes. As well as influencing the overall biogeography of the region, these processes also create special habitat types that are inhabited by marine organisms, such as hydrothermal vents and cold seeps. The changing sea levels during the Pleistocene would mean that the sponge assemblages are likely to have changed composition as different species were better suited to the changing environmental conditions and genera that were found across a wide depth range would have an advantage, such as the twenty most common genera listed in Table 2.2. From the limited fossil record from around New Zealand it has been noted that there was a mixture of present day deepwater genera (Cladorhiza, Chondrocladia, and Hyalonema) and present day shallow water taxa found in the same assemblages from the Cenozoic era, which suggests that they may have coexisted under the same conditions in the past (c.35 million years ago), however, the deep water fauna are not found in the shallow assemblages of today (Hinde \& Holmes, 1892; Kelly et al., 2009). Bryozoan patterns using taxonomic distinctness have been described around New Zealand by Rowden et al., (2004) and these authors also found higher biodiversity values around the Three Kings Islands and in the Foveaux Strait. These are areas of upwelling that will increase productivity and therefore provide an increased availability of food to suspension feeding organisms such as sponges and bryozoans. The areas with high values of AvTD and VarTD for sponge diversity in the Cook Strait, Fiordland and the Foveaux Strait match the patterns of spatial diversity for a combined data set of a wide range of marine organisms (including, wading birds, arthropods, echinoderms, polychaetes, bryozoans, sponges, fish, algae and molluscs) (Beaumont et al., 2010; Costello et al., 2010),

New Zealand waters harbour over 800 shallow water sponge species which is almost $10 \%$ of all the known sponges on a global scale (van Soest et al., 2008). 
Sponge biodiversity patterns were included in a recent report mapping New Zealand coastal waters to $12 \mathrm{~nm}$ offshore or to $250 \mathrm{~m}$ depth (Beaumont et al., 2010). Beaumont et al. combined attributed values of sponge diversity (total records, total species, species richness, AvTD, VarTD, species rarity and species composition) in one ranked value (Fig. 2.15).

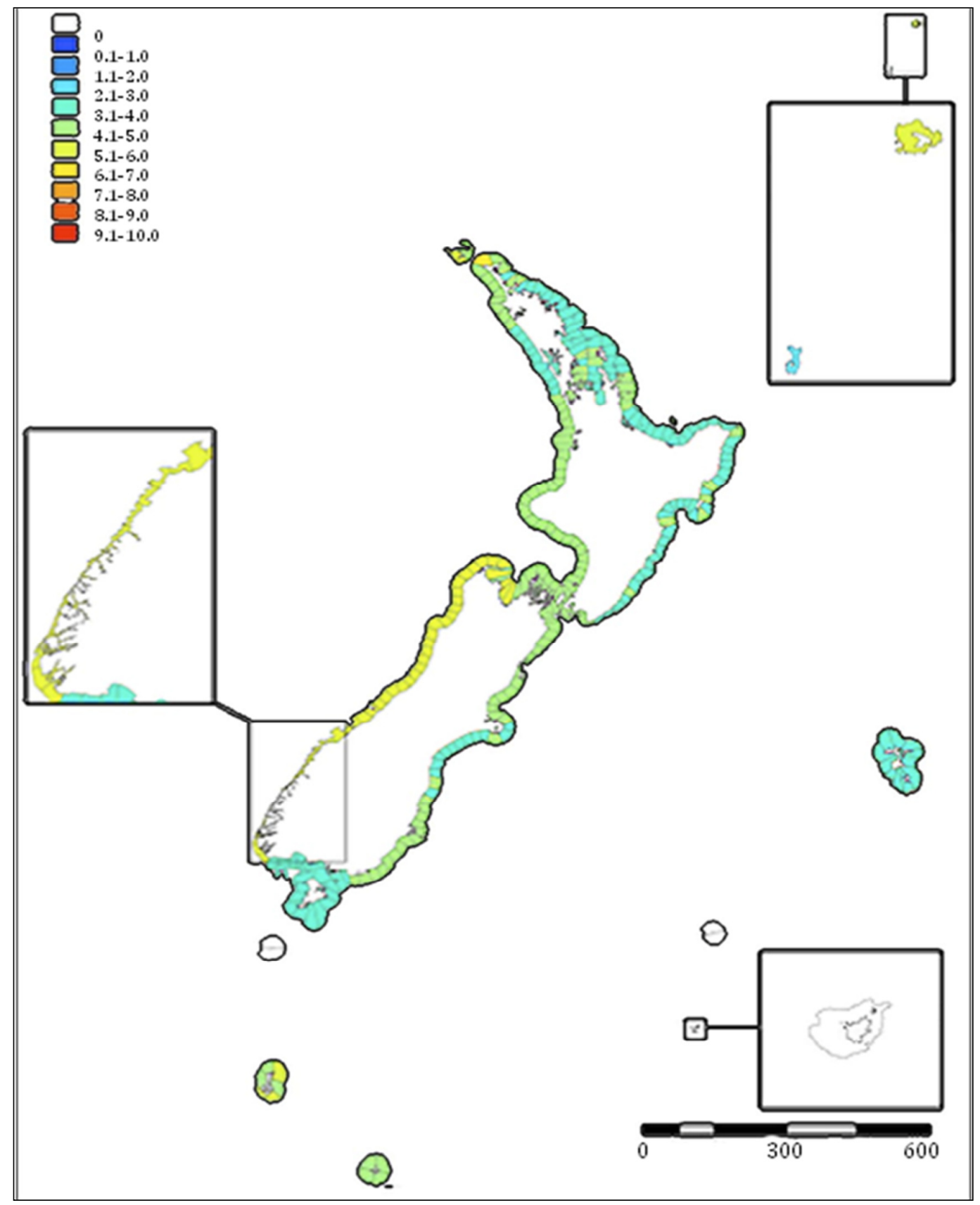


Fig. 2.15 Spatial distribution sponges in New Zealand using a mean rank of six biodiversity measures. This is taken from Beaumont et al., (2010). It uses a range of biodiversity measures including: total records, total species, species richness, AvTD, VarTD, species rarity and species composition. It takes a mean ranked value for each cell and coastal cells were merged with adjacent cell where there were not sufficient data.

There are large areas of New Zealand, such as the west coast of the South Island, which are still to be surveyed. Beaumont et al. (2010) suggests that the northern tip of New Zealand between Cape Reinga and North Cape has the highest mean rank values. When all the data from $0-200 \mathrm{~m}$ is combined then the Three Kings area (in particular) and the Northeastern region had higher values for VarTD when the data is collated from $0-200 \mathrm{~m}$ compared with $0-50 \mathrm{~m}$ depth.

When the data for this chapter were modelled at the $0.5^{\circ} \times 0.5^{\circ}$ Latitude and Longitude scale, the northern tip of New Zealand had some of the lowest AvTD and VarTD predicted values. The reasons for the discrepancies between this study and Beaumont et al. (2010) are partly that the ranked values in Beaumont et al.'s study incorporated a range of biodiversity measures to find an average value. Some of the biodiversity measures such as species richness and total species would have ranked higher because the Northeastern region has been studied more intensively then other regions around New Zealand (Cryer et al., 2000). Therefore more species have been identified from that area in part because there are lots of species but also due to intensive sampling effort in the Cape Reinga to North Cape area. The high values of species richness would have negated the fact that the values of AvTD and VarTD were not as high as other areas in New Zealand. On closer examination of the list of species recorded by Cryer et al. (2000), the species are distributed across ten orders compared with the twenty orders recorded around New Zealand, however, there are many specious genera such as Dysidea with 9 species and Haliclona with 12 species. Many of the species listed from the NIWA database have since been recategorised; for example in Cryer et al. (2000) there are two species listed within the now defunct genus Orina and two within Sigmadocia, all of which are now included within the Haliclona genus. The northeast of New Zealand also 
escaped the Pleistocene glaciations therefore there has been time for new species to evolve in that area within genera while areas of higher VarTD could be the result of multiple re-colonisations after sea level changes.

From the MDS plots there was a clear shift in the biodiversity of sponge assemblages with depth; however, a combination of depth and location was responsible for about 50\% of the variation in the AvTD and VarTD of the sponge assemblages around New Zealand. The patterns of sponge genera presences within bioregions had a similar relationship to the distances they are apart around the coastlines of New Zealand for the Shears and DOC bioregions.

There are, however limited data in particular from the west coast of the South Island in order to predict specific boundaries and there was no correlation between the $0.5^{\circ}$ by $0.5^{\circ}$ lat, long squares and either bioregion designation scheme. The spatial units from $10 \mathrm{~m}$ or shallower had the widest ranging values of AvTD. The wider range of AvTD values in shallower waters are likely in part to be due wider diversity of habitats sampled in shallower waters. AvTD is robust over a wide range of sampling effort, which is why it is a good biodiversity measure in particular for studies like this one using presence only data therefore sampling effort is not likely to be the main reason for the variability in AvTD (Clarke \& Warwick, 1998; Clarke et al., 2006a). Shallow habitats also have a wider range of environmental variables such as light intensity, sedimentation, nutrient availability, habitat complexity and higher levels of disturbance, which would lead to a wider variety of potential habitat niches available. These local environmental gradients are likely to be responsible for the other $50 \%$ of variability not explained by location and depth.

Environmental factors have been used to explain the distributions of sponges in other regions (Wilkinson \& Cheshire, 1989; Bell \& Barnes, 2000b, c; Hooper et al., 2002; Carballo \& Nava, 2007; de Voogd \& Cleary, 2007). Factors such as nutrient availability are likely to be important for small-scale gradients, however, local recruitment and connectivity, local geomorphology associated 
with niche specialisation are probably important for determining biodiversity gradients at local scales (Hooper et al., 2002). The highest values for AvTD were from some of the spatial units in the Cook Strait on the Wellington South Coast, which also had high values of VarTD. The reasons for these high values could be that the hydrology of the Cook region promotes the mixing on the northern southern bioprovince boundary leading to a mix of northern and southern species present in the area.

Populations of other coastal benthic species across a range of phyla have been found to split at the top of the South Island and bottom of the North Island and upwelling is though to play a major role in causing this disjunction through preventing larval dispersal (Ayers \& Waters, 2005; Ross et al., 2009). Upwelling has been suggested as a barrier in other regions, such as Chilie (Poulin et al., 2002) and the United States (Roughgarden et al., 1988). It has been hypothesised that upwelling on the northern east and west coasts of the South Island could deflect larvae from the coastal region into deep water where they will not survive. Weather patterns off mainland New Zealand are highly variable and as upwellings are wind driven their timing and duration can vary (Heath, 1985; de Lange et al., 2003). Not all larvae are transported offshore as the timing of their release into the water column, ability to move up and down and their pelagic duration would mean that larvae would experience upwelling and downwelling and be unaffected in their final settlement location (Shanks \& Shearman, 2009). The actual position of the boundary is thought to be at the top of the South Island close to the Cook Strait on the West Coast (Apte \& Gardner, 2002; Ayers \& Waters, 2005; Jones et al., 2008), while on the East coast the boundary is more variable (Ross et al., 2009). On the East coast the East Cape Current and the Wairarapa Eddy (Fig. 2.1) could at the southern limit prevent northern larvae reaching the southern populations by being deflected back north or out eastwards towards the Chatham Rise. This is not the case for all taxa as some with long larval pelagic duration (8-15 months), such as rock lobsters, and others with shorter larval pelagic duration (2-5 weeks), such as 
the echinoderm Coscinasterias muricata, have no population subdivision around New Zealand (Smith et al., 1980; Waters \& Roy, 2003).

It is thought that most Calcarea and Demospongiae have a larval stage, however, this is not yet known for all families (Maldonado, 2006). Sponge larvae tend to be lecithotrophic with a very short planktonic life lasting from a few minutes to up to two weeks. Some sponges have benthic larvae rather than planktonic larvae, for example Halichondria moorei from New Zealand, which settles and then crawls along the sediment for two to three days (Bergquist \& Sinclair, 1968). This would mean it is unlikely to be dispersed widely from the source sponge. Larval release tends to take place just before or during when the waters are warmest in sub tropical locations (Mariani et al., 2005) and corresponds to the rain/dry seasons in tropical waters (Zea, 1993). In New Zealand different subtidal sponges reproduce at varying times of the year for a range of durations (Ayling, 1980). Some sponges genera such as Tethya and Polymastia can reproduce asexually by budding all year round and therefore there are continuous low levels of recruitment (Bergquist \& Sinclair, 1968; Ayling, 1980). Tethya was the second most widely reported sponge in New Zealand waters and Polymastia was the eleventh. These buds may be able to survive longer and therefore be carried further from the adult sponge particularly in stormy weather (Battershill, 1987). This has also been reported in the tropics for fragments of other adult sponges (Wulff, 1991). Callyspongia was the most commonly reported genera and it is known to asexually reproduce through branch fragmentation in tropical environments so this may also be occurring in temperate species (Leong \& Pawlik, 2010).

This assessment of New Zealand waters is a first attempt to assimilate all the baseline knowledge of sponge biodiversity distribution at a national and regional level. One of the shortcomings of the whole data set is limited distribution of the data as the majority of the species records are from areas close to cities or research institutions or result from specific research 
objectives, such as the port surveys, (potential) marine reserve surveys or on research cruises to a specific area. The distortion of spatial and temporal species occurrence data is a known problem when trying to assemble regional and global data sets from disparate sources (Boakes et al., 2010). Values of AvTD and VarTD are excellent robust methods of comparing occurrence data as long as there are at least ten records from the areas surveyed. More wide ranging surveys are needed to increase spatial coverage and more fully understand the patterns of biodiversity. The main recommendation from this research is for more surveys in areas with limited records such as the West coast of the South Island. In order to survey these areas where there are no or few data available broad scale surveys are necessary. Surveying is expensive and time consuming therefore broad-scale surveying by volunteer divers can be used as a economically viable tool to as long as there is some quality control for the training and data consistency (Foster-Smith \& Evans, 2003). Once the broad scale surveys have identified areas with high sponge abundance and diversity they can provide a priority list of areas where taxonomists are needed to work up the samples from biodiversity hotspots within the bioregions. Areas with values of AvTD and/or VarTD above the values predicted for the $95 \%$ probability funnel have significantly more diverse sponge assemblages and therefore should be candidates for further protection. Marine bioblitz's may be a suitable tool to sample a small area exhaustively in order to create a species list. Mobilising a large team to intensively sample an area of high biodiversity in a short period of time such as the marine bioblitz on the Wellington South Coast in 2007 not only found more than a dozen undescribed species of invertebrates but also created a comprehensive list of over 600 species found in the marine reserve across all phyla (Gordon, 2009; Harper et al., 2009). As well as areas with high biodiversity it is important to consider common, endemic and rare species.

Common species, those that are abundant and widespread, are actually very rare (Gaston, 2010). Yet, common species contribute the majority of the 
structure, biomass, and energy turnover for most ecosystems (Gaston \& Fuller, 2008). There is scant abundance information for sponges in New Zealand and therefore it is difficult from a species list to know which ones are abundant or rare. Common species are often the first to be affected by overexploitation, however, this is not likely to be the case for sponges with habitat degradation likely to have a significant effect on abundances. This chapter did not take into consideration any abundance data as most records were only species lists. However, chapters three and four in this thesis will take abundance into account when examining spatial and temporal variability in sponge assemblages on the Wellington South Coast.

Over 95\% of New Zealand sponge species are thought to be endemic (Kelly et al., 2009). However, the number of taxonomists in New Zealand is at its lowest levels since WWII, therefore there is a significant cross-phyla backlog of species identifications (Bradford-Grieve, 2008). There needs to be an investment in training up taxonomists so that the new and undescribed species, which are still common in New Zealand marine samples can be identified (Gordon et al., 2010). Biodiversity includes more than just species richness encompassing the diversity of genes to ecosystems (Gaston, 2000). It is important to conserve both ecologically and economically intraspecific variation. For example, Mycale hentscheli are found in various locations around New Zealand but it is only Mycale hentscheli in the Pelorus Sound that produces Peloruside A, which is a potential anticancer drug (Page et al., 2005). M. hentscheli also stops producing Peloruside A when they are moved to other locations so the environmental qualities of the Pelorus Sound are vital for the sponge to be able to produce Peloruside A (Page et al., 2005).

Only one sponge species in New Zealand waters (Lissendoryx sp. yellow slimy) has been assessed (as locally uncommon) and assigned within the New Zealand Threat Classification System (Freeman et al., 2010). The other 800 plus sponges have not yet been assessed. Fifty of the genera from this study were recorded as present in only one $0.5^{\circ} \times 0.5^{\circ}$ lat/long area in New Zealand waters. As the data 
available is sparse it is hard to predict how many of those genera are naturally uncommon and only found in one specific location habitat type or if they simply have not been looked for. Rare species often have poorer dispersal abilities, as they have a tendency toward asexual reproductive pathways and lower reproductive effort (Kunin \& Gaston, 1993). Therefore rare species often remain naturally rare and are particularly vulnerable to habitat loss or damage. Rare species are often restricted to specific habitat characteristics, suggesting an important link between habitat diversity and rarity (Ellingsen et al., 2007). Rare species are not only important to biodiversity but could play a role in driving assemblage dynamics in fluctuating environments (Benedetti-Cecchi et al., 2008). Therefore it is important have good baseline species level data which can then be repetitively surveyed over time using genera level data in order to monitor for any changes in the sponge assemblages.

This work provides an initial overview of the distribution of sponge biodiversity in New Zealand waters. The limitations are that there are large gaps in the data set and that the data have been collecting using a wide variety of methods over a long time period. AvTD and VarTD values provide an opportunity to compare aspects of biodiversity but there needs to be at least ten species recorded from an area in order to use them as a tool. The focus of chapter 3 will be on the Wellington region where there are the high values of AvTD and VarTD and also this area is considered as a potential bioregion. Initially, the local spatial diversity will be assessed and then in chapter 4 the temporal variation will be assessed. Ideally with more time and funding the first step in progressing the work from this chapter would be further structured biodiversity surveys in the areas where there is missing data from around New Zealand. 


\subsection{APPENDIX OF THE MODEL FITTING FOR SPONGE BIODIVERSITY AROUND NEW ZEALAND FOR AVTD AND VARTD VALUES}

Table 2.3 Values for AvTD and VarTD for sponge assemblages with more than ten genera present in New Zealand waters.

\begin{tabular}{|r|r|r|r|r|}
\hline Depth & Latitude & Longitude & AvTD & VarTD \\
\hline 2.5 & 35.5 & 174.5 & 71.01 & 126.24 \\
\hline 2.5 & 35.5 & 175 & 69.19 & 230.13 \\
\hline 2.5 & 36 & 174.5 & 71.59 & 151.42 \\
\hline 2.5 & 36.5 & 174.5 & 71.01 & 147.23 \\
\hline 2.5 & 36.5 & 175 & 72.81 & 135.74 \\
\hline 2.5 & 37 & 174.5 & 69.81 & 240.66 \\
\hline 2.5 & 37 & 175 & 71.97 & 149.03 \\
\hline 2.5 & 37 & 176 & 80.28 & 246.59 \\
\hline 2.5 & 37.5 & 176.5 & 71.56 & 164.12 \\
\hline 2.5 & 39 & 174.5 & 74.41 & 170.32 \\
\hline 2.5 & 40 & 173.5 & 72.23 & 119.46 \\
\hline 2.5 & 40 & 174.5 & 76.8 & 166.81 \\
\hline 2.5 & 41 & 174.5 & 83.21 & 362.52 \\
\hline 2.5 & 41 & 175 & 85.97 & 333.9 \\
\hline 2.5 & 44.5 & 176 & 72.02 & 163.69 \\
\hline 7.5 & 35.5 & 174.5 & 74.27 & 214.48 \\
\hline 7.5 & 36 & 174.5 & 71.47 & 164.17 \\
\hline 7.5 & 36.5 & 175 & 68.06 & 203.47 \\
\hline 7.5 & 37 & 175 & 79.87 & 227.46 \\
\hline 7.5 & 37.5 & 176 & 68.32 & 192.69 \\
\hline 7.5 & 38.5 & 178 & 79.38 & 201.14 \\
\hline 7.5 & 39 & 174 & 77.2 & 231.54 \\
\hline 7.5 & 40.5 & 173.5 & 73.17 & 113.15 \\
\hline 7.5 & 41 & 174 & 67.59 & 196.9 \\
\hline 7.5 & 43.5 & 172.5 & 76.5 & 238.73 \\
\hline 7.5 & 44 & 171.5 & 76.95 & 296.96 \\
\hline 7.5 & 45.5 & 170.5 & 77.06 & 243.8 \\
\hline 7.5 & 46.5 & 168 & 79.6 & 278.17 \\
\hline 12.5 & 34 & 172 & 74.36 & 248.12 \\
\hline 12.5 & 35.5 & 174.5 & 72.05 & 127.06 \\
\hline 12.5 & 36 & 174.5 & 71.86 & 125.86 \\
\hline 12.5 & 36 & 175 & 80.27 & 300.25 \\
\hline 12.5 & 36 & 175.5 & 67.6 & 182.81 \\
\hline
\end{tabular}




\begin{tabular}{|c|c|c|c|c|}
\hline Depth & Latitude & Longitude & AvTD & VarTD \\
\hline 12.5 & 36.5 & 175 & 72.36 & 109.57 \\
\hline 12.5 & 38 & 178 & 77.56 & 249.49 \\
\hline 12.5 & 39 & 174 & 78.73 & 184.09 \\
\hline 12.5 & 41 & 174.5 & 79.81 & 218.08 \\
\hline 17.5 & 34.5 & 173 & 72.48 & 303.96 \\
\hline 17.5 & 35.5 & 174.5 & 70.37 & 140.33 \\
\hline 17.5 & 36 & 174.5 & 74.76 & 210.66 \\
\hline 17.5 & 36 & 175 & 70.75 & 122 \\
\hline 17.5 & 36.5 & 175 & 67.85 & 186.71 \\
\hline 17.5 & 37.5 & 178 & 76.22 & 248.12 \\
\hline 17.5 & 38.5 & 178 & 74.98 & 279.56 \\
\hline 17.5 & 40.5 & 174.5 & 75.39 & 222.73 \\
\hline 17.5 & 42.5 & 173.5 & 70.78 & 349.57 \\
\hline 17.5 & 44.5 & 167.5 & 78.71 & 294.8 \\
\hline 25 & 34 & 172.5 & 66.93 & 171.92 \\
\hline 25 & 34 & 172.5 & 73.93 & 263.58 \\
\hline 25 & 34 & 173 & 69.83 & 162.76 \\
\hline 25 & 35.5 & 174.5 & 71.5 & 115.42 \\
\hline 25 & 36 & 174.5 & 74.37 & 219.95 \\
\hline 25 & 37 & 176 & 72.56 & 130.78 \\
\hline 35 & 34 & 172.5 & 74.02 & 205.91 \\
\hline 35 & 34 & 173 & 68.46 & 168.26 \\
\hline 35 & 34.5 & 173 & 71.97 & 202.49 \\
\hline 35 & 36 & 174.5 & 73.25 & 135.92 \\
\hline 45 & 34 & 172.5 & 74.28 & 192.71 \\
\hline 45 & 34.5 & 172.5 & 72.08 & 191.03 \\
\hline 45 & 34.5 & 173 & 73.78 & 177.04 \\
\hline 55 & 34 & 172.5 & 72.87 & 161.29 \\
\hline 55 & 34.5 & 172.5 & 72.31 & 169.91 \\
\hline 55 & 34.5 & 173 & 72.84 & 172.07 \\
\hline 65 & 34 & 172.5 & 73.58 & 195.77 \\
\hline 65 & 34.5 & 172 & 70.52 & 226.41 \\
\hline 65 & 34.5 & 172.5 & 69.06 & 169.31 \\
\hline 65 & 34.5 & 173 & 74.15 & 195.69 \\
\hline 65 & 36 & 175.5 & 70.41 & 131.78 \\
\hline 75 & 34 & 172.5 & 72.53 & 227.86 \\
\hline 75 & 34.5 & 172.5 & 74.65 & 203.75 \\
\hline 75 & 34.5 & 173 & 67.98 & 168.14 \\
\hline 75 & 35.5 & 174.5 & 72.77 & 144.27 \\
\hline 85 & 34 & 172.5 & 71.36 & 194.26 \\
\hline
\end{tabular}




\begin{tabular}{|r|r|r|r|r|}
\hline Depth & Latitude & Longitude & AvTD & VarTD \\
\hline 85 & 34 & 173 & 70.48 & 153.78 \\
\hline 85 & 34.5 & 172.5 & 75.25 & 250.92 \\
\hline 85 & 34.5 & 173 & 74.18 & 221.37 \\
\hline 95 & 34 & 172 & 70.72 & 275.58 \\
\hline 95 & 34 & 173 & 72.07 & 131.38 \\
\hline 110 & 34 & 173 & 76.76 & 271.22 \\
\hline 130 & 34.5 & 173 & 75.31 & 282.94 \\
\hline 130 & 37.5 & 177 & 68.87 & 254.68 \\
\hline 150 & 34 & 172 & 75.58 & 278.05 \\
\hline 150 & 34 & 172.5 & 71.25 & 125.64 \\
\hline 150 & 34.5 & 172 & 70.42 & 249.13 \\
\hline 150 & 34.5 & 173 & 69.6 & 225.89 \\
\hline 150 & 37.5 & 177 & 72.93 & 227.07 \\
\hline
\end{tabular}

Table 2.4 AvTD models for $150 \mathrm{~m}$ or shallower sorted in ascending order of AIC (lowest AIC is the best model)

\begin{tabular}{|c|c|c|c|c|c|c|c|c|}
\hline $\begin{array}{l}\text { No. of factors } \\
\text { used }\end{array}$ & 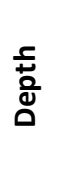 & 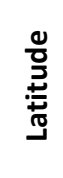 & 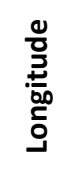 & 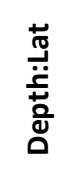 & 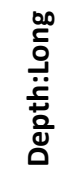 & مَّ & AIC & Likelihood \\
\hline 4 & $\mathrm{n}$ & y & $\mathrm{n}$ & $y$ & $y$ & $\mathrm{y}$ & 420.5288 & -192.8898 \\
\hline 5 & $\mathrm{y}$ & $\mathrm{y}$ & $\mathrm{n}$ & $\mathrm{y}$ & $\mathrm{y}$ & $\mathrm{y}$ & 420.6146 & -189.4222 \\
\hline 3 & $\mathrm{n}$ & $\mathrm{n}$ & $y$ & $y$ & $\mathrm{n}$ & $\mathrm{y}$ & 422.7302 & -191.5344 \\
\hline 3 & $\mathrm{y}$ & $\mathrm{n}$ & $\mathrm{n}$ & $\mathrm{n}$ & y & y & 422.904 & -189.7611 \\
\hline 2 & $\mathrm{n}$ & $\mathrm{n}$ & $\mathrm{n}$ & $\mathrm{n}$ & y & $\mathrm{y}$ & 424.7238 & -189.7627 \\
\hline 6 & $\mathrm{y}$ & y & y & $\mathrm{y}$ & y & $\mathrm{y}$ & 425.2432 & -202.1123 \\
\hline 5 & $\mathrm{n}$ & $\mathrm{y}$ & $\mathrm{y}$ & $\mathrm{y}$ & y & $\mathrm{y}$ & 426.76 & -201.3985 \\
\hline 4 & $\mathrm{y}$ & y & $n$ & $n$ & y & y & 427.1592 & -196.8837 \\
\hline 4 & $\mathrm{y}$ & $n$ & $\mathrm{n}$ & $y$ & y & $\mathrm{y}$ & 427.265 & -203.2327 \\
\hline 4 & $\mathrm{n}$ & $\mathrm{y}$ & $\mathrm{y}$ & $\mathrm{n}$ & $\mathrm{y}$ & $\mathrm{y}$ & 427.8269 & -199.2439 \\
\hline 4 & $\mathrm{y}$ & $n$ & y & $y$ & $n$ & $\mathrm{y}$ & 427.911 & -200.0506 \\
\hline 4 & $\mathrm{n}$ & $\mathrm{n}$ & $\mathrm{y}$ & y & $\mathrm{y}$ & $\mathrm{y}$ & 428.0065 & -200.1049 \\
\hline 5 & y & y & y & $\mathrm{n}$ & y & y & 428.0816 & -198.9884 \\
\hline 3 & $\mathrm{n}$ & $\mathrm{y}$ & $\mathrm{y}$ & $\mathrm{n}$ & $\mathrm{n}$ & y & 428.1038 & -200.3435 \\
\hline 4 & $\mathrm{n}$ & $\mathrm{y}$ & $\mathrm{y}$ & y & $\mathrm{n}$ & $\mathrm{y}$ & 428.2775 & -201.7518 \\
\hline 4 & $\mathrm{y}$ & $\mathrm{n}$ & y & $\mathrm{n}$ & y & y & 428.4553 & -198.6529 \\
\hline 3 & $y$ & $\mathrm{n}$ & $\mathrm{n}$ & y & $\mathrm{n}$ & y & 428.4989 & -207.9348 \\
\hline 3 & $\mathrm{y}$ & $\mathrm{n}$ & $\mathrm{y}$ & y & $\mathrm{n}$ & $\mathrm{n}$ & 428.6097 & -207.9427 \\
\hline 5 & $\mathrm{y}$ & $\mathrm{y}$ & $\mathrm{y}$ & y & $\mathrm{n}$ & $\mathrm{y}$ & 428.7935 & -201.9095 \\
\hline
\end{tabular}




\begin{tabular}{|c|c|c|c|c|c|c|c|c|}
\hline $\begin{array}{c}\text { No. of factors } \\
\text { used }\end{array}$ & $\begin{array}{l}\frac{5}{0} \\
\text { 口 }\end{array}$ & 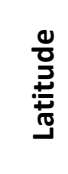 & 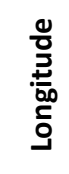 & 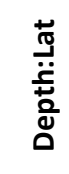 & 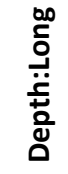 & 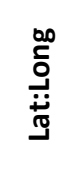 & AIC & Likelihood \\
\hline 5 & $\mathrm{y}$ & $\mathrm{n}$ & $\mathrm{y}$ & $\mathrm{y}$ & $\mathrm{y}$ & $\mathrm{y}$ & 428.8693 & -198.5042 \\
\hline 4 & $\mathrm{n}$ & $y$ & $y$ & $y$ & $y$ & $\mathrm{n}$ & 428.9077 & -201.4736 \\
\hline 2 & $\mathrm{n}$ & $\mathrm{n}$ & $\mathrm{n}$ & $\mathrm{y}$ & $\mathrm{n}$ & $\mathrm{y}$ & 429.0076 & -200.0388 \\
\hline 2 & $\mathrm{n}$ & $\mathrm{n}$ & $\mathrm{y}$ & $\mathrm{n}$ & $\mathrm{n}$ & $\mathrm{y}$ & 429.0398 & -199.8984 \\
\hline 4 & $\mathrm{y}$ & $\mathrm{n}$ & $\mathrm{y}$ & $\mathrm{y}$ & $y$ & $\mathrm{n}$ & 429.1533 & -202.06 \\
\hline 3 & $\mathrm{n}$ & $\mathrm{y}$ & $\mathrm{n}$ & $\mathrm{y}$ & $\mathrm{n}$ & $\mathrm{y}$ & 429.4198 & -200.7233 \\
\hline 3 & $\mathrm{n}$ & $\mathrm{n}$ & $\mathrm{y}$ & $\mathrm{n}$ & $y$ & $\mathrm{y}$ & 429.551 & -201.3553 \\
\hline 4 & $\mathrm{y}$ & $\mathrm{y}$ & $\mathrm{n}$ & $\mathrm{y}$ & $\mathrm{n}$ & $\mathrm{y}$ & 429.5746 & -206.5186 \\
\hline 3 & $\mathrm{y}$ & $\mathrm{n}$ & $\mathrm{n}$ & $y$ & $y$ & $\mathrm{n}$ & 429.6135 & -209.3513 \\
\hline 5 & $\mathrm{y}$ & $\mathrm{y}$ & $\mathrm{y}$ & $\mathrm{y}$ & $\mathrm{y}$ & $\mathrm{n}$ & 429.7518 & -207.7197 \\
\hline 4 & $y$ & $\mathrm{y}$ & $\mathrm{y}$ & $\mathrm{n}$ & $\mathrm{n}$ & $\mathrm{y}$ & 429.9012 & -200.2876 \\
\hline 3 & $\mathrm{n}$ & $y$ & $\mathrm{n}$ & $y$ & $y$ & $\mathrm{n}$ & 429.9159 & -207.3605 \\
\hline 3 & $\mathrm{n}$ & $\mathrm{n}$ & $\mathrm{n}$ & $\mathrm{y}$ & $\mathrm{y}$ & $\mathrm{y}$ & 430.0029 & -206.3687 \\
\hline 2 & $y$ & $\mathrm{n}$ & $\mathrm{n}$ & $y$ & $\mathrm{n}$ & $\mathrm{n}$ & 430.0425 & -210.1154 \\
\hline 2 & $\mathrm{n}$ & $\mathrm{y}$ & $\mathrm{n}$ & $\mathrm{y}$ & $\mathrm{n}$ & $\mathrm{n}$ & 430.2324 & -208.8725 \\
\hline 4 & $y$ & $y$ & $\mathrm{n}$ & $y$ & $y$ & $\mathrm{n}$ & 430.2581 & -208.2388 \\
\hline 4 & $\mathrm{y}$ & $y$ & $\mathrm{y}$ & $\mathrm{y}$ & $\mathrm{n}$ & $\mathrm{n}$ & 430.3858 & -207.7509 \\
\hline 2 & $\mathrm{n}$ & $\mathrm{n}$ & $\mathrm{y}$ & $y$ & $\mathrm{n}$ & $\mathrm{n}$ & 430.6539 & -207.1491 \\
\hline 3 & $\mathrm{n}$ & $y$ & $\mathrm{y}$ & $y$ & $\mathrm{n}$ & $\mathrm{n}$ & 430.7476 & -208.0335 \\
\hline 2 & $\mathrm{n}$ & $\mathrm{n}$ & $\mathrm{n}$ & $y$ & $y$ & $\mathrm{n}$ & 430.7588 & -209.2914 \\
\hline 1 & $\mathrm{n}$ & $\mathrm{n}$ & $\mathrm{n}$ & $\mathrm{n}$ & $\mathrm{n}$ & $y$ & 430.8415 & -201.8462 \\
\hline 2 & $\mathrm{n}$ & $\mathrm{y}$ & $\mathrm{n}$ & $\mathrm{n}$ & $\mathrm{n}$ & $y$ & 430.8415 & -201.8462 \\
\hline 3 & $y$ & $\mathrm{n}$ & $y$ & $\mathrm{n}$ & $\mathrm{n}$ & $y$ & 430.8484 & -199.9157 \\
\hline 3 & $\mathrm{y}$ & $y$ & $\mathrm{n}$ & $y$ & $\mathrm{n}$ & $\mathrm{n}$ & 430.958 & -210.0355 \\
\hline 1 & $\mathrm{n}$ & $\mathrm{n}$ & $\mathrm{n}$ & $y$ & $\mathrm{n}$ & $\mathrm{n}$ & 431.0873 & -209.8605 \\
\hline 3 & $\mathrm{n}$ & $y$ & $\mathrm{y}$ & $\mathrm{n}$ & $\mathrm{y}$ & $\mathrm{n}$ & 431.5431 & -201.7127 \\
\hline 1 & $\mathrm{n}$ & $y$ & $\mathrm{n}$ & $\mathrm{n}$ & $\mathrm{n}$ & $\mathrm{n}$ & 431.7191 & -212.859 \\
\hline 4 & $\mathrm{y}$ & $y$ & $\mathrm{y}$ & $\mathrm{n}$ & $y$ & $\mathrm{n}$ & 432.2745 & -208.2764 \\
\hline 2 & $\mathrm{n}$ & $y$ & $\mathrm{n}$ & $\mathrm{n}$ & $y$ & $\mathrm{n}$ & 432.275 & -208.2766 \\
\hline 3 & $y$ & $y$ & $\mathrm{n}$ & $\mathrm{n}$ & $y$ & $\mathrm{n}$ & 432.275 & -208.2766 \\
\hline 3 & $\mathrm{y}$ & $\mathrm{y}$ & $\mathrm{n}$ & $\mathrm{n}$ & $\mathrm{n}$ & $\mathrm{y}$ & 432.5113 & -201.1935 \\
\hline 2 & $\mathrm{y}$ & $\mathrm{n}$ & $\mathrm{n}$ & $\mathrm{n}$ & $\mathrm{n}$ & $\mathrm{y}$ & 432.8654 & -208.3557 \\
\hline 3 & $\mathrm{n}$ & $y$ & $\mathrm{n}$ & $\mathrm{n}$ & $y$ & y & 432.8665 & -208.3558 \\
\hline 2 & $\mathrm{n}$ & $\mathrm{y}$ & $\mathrm{y}$ & $\mathrm{n}$ & $\mathrm{n}$ & $\mathrm{n}$ & 433.0275 & -210.4338 \\
\hline 3 & $\mathrm{n}$ & $\mathrm{n}$ & $\mathrm{y}$ & $y$ & $\mathrm{y}$ & $\mathrm{n}$ & 433.1732 & -210.2668 \\
\hline 2 & $\mathrm{y}$ & $\mathrm{y}$ & $\mathrm{n}$ & $\mathrm{n}$ & $\mathrm{n}$ & $\mathrm{n}$ & 433.6302 & -212.8151 \\
\hline 3 & $\mathrm{y}$ & $\mathrm{y}$ & $\mathrm{y}$ & $\mathrm{n}$ & $\mathrm{n}$ & $\mathrm{n}$ & 434.9665 & -210.5568 \\
\hline
\end{tabular}




\begin{tabular}{|c|c|c|c|c|c|c|c|c|}
\hline $\begin{array}{c}\text { No. of factors } \\
\text { used }\end{array}$ & 章 & 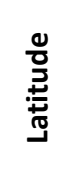 & 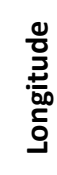 & 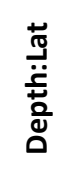 & 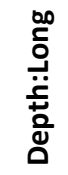 & مِّ & AIC & Likelihood \\
\hline 2 & $\mathrm{y}$ & $\mathrm{n}$ & $\mathrm{n}$ & $\mathrm{n}$ & $\mathrm{y}$ & $\mathrm{n}$ & 441.5985 & -213.8919 \\
\hline 3 & $\mathrm{y}$ & $\mathrm{n}$ & $y$ & $\mathrm{n}$ & $\mathrm{y}$ & $n$ & 441.5986 & -213.892 \\
\hline 2 & $\mathrm{y}$ & $\mathrm{n}$ & $\mathrm{y}$ & $\mathrm{n}$ & $\mathrm{n}$ & $\mathrm{n}$ & 442.3134 & -215.487 \\
\hline 1 & $\mathrm{n}$ & $\mathrm{n}$ & $\mathrm{n}$ & $\mathrm{n}$ & $y$ & $\mathrm{n}$ & 442.3763 & -214.2165 \\
\hline 2 & $\mathrm{n}$ & $\mathrm{n}$ & $\mathrm{y}$ & $\mathrm{n}$ & $\mathrm{y}$ & $\mathrm{n}$ & 442.3763 & -214.2165 \\
\hline 1 & $\mathrm{n}$ & $\mathrm{n}$ & $\mathrm{y}$ & $\mathrm{n}$ & $\mathrm{n}$ & $\mathrm{n}$ & 443.34 & -217.1636 \\
\hline 1 & $y$ & $\mathrm{n}$ & $\mathrm{n}$ & $\mathrm{n}$ & $\mathrm{n}$ & $\mathrm{n}$ & 445.2692 & -219.1761 \\
\hline
\end{tabular}

Table 2.5 AvTD models for $50 \mathrm{~m}$ or shallower (lowest AIC is the best model)

\begin{tabular}{|c|c|c|c|l|r|}
\hline \multirow{2}{*}{ No. of factors used } & \multicolumn{4}{l}{ Latitude } & \multicolumn{2}{l|}{ Longitude } & \multicolumn{1}{l|}{ Lat:Long } & AIC & Likelihood \\
\hline 1 & $\mathrm{y}$ & $\mathrm{n}$ & $\mathrm{n}$ & 330.3068 & -162.116 \\
\hline 1 & $\mathrm{n}$ & $\mathrm{y}$ & $\mathrm{n}$ & 339.3749 & -165.587 \\
\hline 1 & $\mathrm{n}$ & $\mathrm{n}$ & $\mathrm{y}$ & 329.7106 & -153.114 \\
\hline 2 & $\mathrm{y}$ & $\mathrm{y}$ & $\mathrm{n}$ & 331.4645 & -160.171 \\
\hline 2 & $\mathrm{y}$ & $\mathrm{n}$ & $\mathrm{y}$ & 329.7106 & -153.114 \\
\hline 2 & $\mathrm{n}$ & $\mathrm{y}$ & $\mathrm{y}$ & 328.4711 & -158.802 \\
\hline 3 & $\mathrm{y}$ & $\mathrm{y}$ & $\mathrm{y}$ & 327.2107 & -152.298 \\
\hline
\end{tabular}

Table 2.6 VarTD models for $150 \mathrm{~m}$ or shallower sorted in ascending order of AIC (lowest AIC is the best model)

\begin{tabular}{|c|c|c|c|c|c|c|c|c|}
\hline No. of factors used & $\frac{\frac{c}{0}}{\frac{0}{0}}$ & & 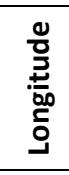 & 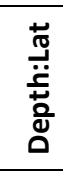 & 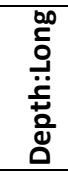 & مَّ & AIC & Likelihood \\
\hline 6 & $\mathrm{y}$ & $\mathrm{y}$ & $\mathrm{y}$ & $\mathrm{y}$ & $\mathrm{y}$ & $\mathrm{y}$ & 854.3409 & -409.305 \\
\hline 5 & $\mathrm{n}$ & $\mathrm{y}$ & $\mathrm{y}$ & $\mathrm{y}$ & $\mathrm{y}$ & y & 854.8198 & -409.859 \\
\hline 4 & $\mathrm{n}$ & $\mathrm{y}$ & $\mathrm{n}$ & $\mathrm{y}$ & $\mathrm{y}$ & $\mathrm{y}$ & 856.3294 & -407.335 \\
\hline 5 & $\mathrm{y}$ & $\mathrm{y}$ & $\mathrm{y}$ & $\mathrm{y}$ & $\mathrm{n}$ & $\mathrm{y}$ & 858.5799 & -409.059 \\
\hline 3 & $\mathrm{n}$ & $\mathrm{y}$ & $\mathrm{n}$ & $\mathrm{y}$ & $\mathrm{n}$ & $\mathrm{y}$ & 859.2344 & -415.22 \\
\hline 4 & $\mathrm{n}$ & $\mathrm{y}$ & $\mathrm{y}$ & $\mathrm{y}$ & $\mathrm{n}$ & $\mathrm{y}$ & 859.2853 & -411.57 \\
\hline 4 & $\mathrm{y}$ & $\mathrm{y}$ & $\mathrm{y}$ & $\mathrm{n}$ & $\mathrm{n}$ & $\mathrm{y}$ & 859.8232 & -409.853 \\
\hline 5 & $\mathrm{y}$ & $\mathrm{y}$ & $\mathrm{y}$ & $\mathrm{n}$ & $\mathrm{y}$ & $\mathrm{y}$ & 859.8232 & -409.853 \\
\hline 5 & $\mathrm{y}$ & $\mathrm{y}$ & $\mathrm{n}$ & $\mathrm{y}$ & $\mathrm{y}$ & $\mathrm{y}$ & 860.2458 & -414.982 \\
\hline 3 & $\mathrm{y}$ & $\mathrm{y}$ & $\mathrm{n}$ & $\mathrm{n}$ & $\mathrm{n}$ & $\mathrm{y}$ & 860.505 & -415.33 \\
\hline 4 & $\mathrm{n}$ & $\mathrm{y}$ & $\mathrm{y}$ & $\mathrm{y}$ & $\mathrm{y}$ & $\mathrm{n}$ & 860.5141 & -414.515 \\
\hline
\end{tabular}




\begin{tabular}{|c|c|c|c|c|c|c|c|c|}
\hline No. of factors used & $\begin{array}{l}\frac{5}{0} \\
\frac{0}{0}\end{array}$ & 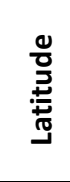 & 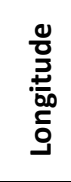 & 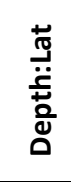 & 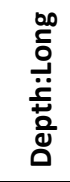 & 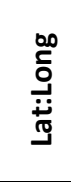 & AIC & Likelihood \\
\hline 3 & $\mathrm{n}$ & $\mathrm{y}$ & $\mathrm{y}$ & $\mathrm{y}$ & $\mathrm{n}$ & $\mathrm{n}$ & 860.5222 & -414.522 \\
\hline 4 & $\mathrm{n}$ & $\mathrm{y}$ & $\mathrm{y}$ & $\mathrm{n}$ & $\mathrm{y}$ & $\mathrm{y}$ & 861.079 & -410.163 \\
\hline 4 & $\mathrm{y}$ & $\mathrm{y}$ & $\mathrm{n}$ & $\mathrm{n}$ & $\mathrm{y}$ & $\mathrm{y}$ & 861.3122 & -409.103 \\
\hline 4 & $\mathrm{y}$ & $\mathrm{y}$ & $\mathrm{n}$ & $\mathrm{y}$ & $\mathrm{n}$ & $\mathrm{y}$ & 861.5538 & -415.179 \\
\hline 2 & $\mathrm{y}$ & $\mathrm{n}$ & $\mathrm{n}$ & $\mathrm{n}$ & $\mathrm{n}$ & $\mathrm{y}$ & 862.408 & -418.296 \\
\hline 3 & $\mathrm{y}$ & $\mathrm{n}$ & $\mathrm{y}$ & $\mathrm{n}$ & $\mathrm{n}$ & $\mathrm{y}$ & 862.408 & -418.296 \\
\hline 5 & $\mathrm{y}$ & $\mathrm{n}$ & y & $\mathrm{y}$ & $\mathrm{y}$ & $\mathrm{y}$ & 862.408 & -418.296 \\
\hline 3 & $\mathrm{y}$ & $\mathrm{y}$ & $\mathrm{y}$ & $\mathrm{n}$ & $\mathrm{n}$ & $\mathrm{n}$ & 862.9223 & -414.213 \\
\hline 4 & $\mathrm{y}$ & $\mathrm{y}$ & $\mathrm{y}$ & $\mathrm{y}$ & $\mathrm{n}$ & $\mathrm{n}$ & 862.9223 & -414.213 \\
\hline 5 & $\mathrm{y}$ & $\mathrm{y}$ & $\mathrm{y}$ & $\mathrm{y}$ & $\mathrm{y}$ & $\mathrm{n}$ & 862.9223 & -414.213 \\
\hline 3 & $\mathrm{n}$ & $\mathrm{y}$ & $\mathrm{n}$ & $\mathrm{n}$ & $\mathrm{y}$ & $\mathrm{y}$ & 863.1555 & -418.249 \\
\hline 4 & $\mathrm{y}$ & $\mathrm{n}$ & $\mathrm{n}$ & $\mathrm{y}$ & $\mathrm{y}$ & $\mathrm{y}$ & 863.9176 & -416.922 \\
\hline 3 & $\mathrm{n}$ & $\mathrm{n}$ & $\mathrm{n}$ & $\mathrm{y}$ & $\mathrm{y}$ & $\mathrm{y}$ & 864.2107 & -416.892 \\
\hline 3 & $\mathrm{y}$ & $\mathrm{n}$ & $\mathrm{n}$ & $\mathrm{n}$ & $\mathrm{y}$ & $\mathrm{y}$ & 864.2969 & -419.785 \\
\hline 4 & $\mathrm{y}$ & $\mathrm{y}$ & $\mathrm{y}$ & $\mathrm{n}$ & $\mathrm{y}$ & $\mathrm{n}$ & 864.4606 & -414.344 \\
\hline 4 & $\mathrm{y}$ & $\mathrm{n}$ & $\mathrm{y}$ & $\mathrm{n}$ & $\mathrm{y}$ & $\mathrm{y}$ & 864.4974 & -417.352 \\
\hline 4 & $\mathrm{y}$ & $\mathrm{n}$ & $\mathrm{y}$ & $\mathrm{y}$ & $\mathrm{n}$ & $\mathrm{y}$ & 864.7447 & -418.476 \\
\hline 3 & $\mathrm{n}$ & $\mathrm{y}$ & $\mathrm{y}$ & $\mathrm{n}$ & $\mathrm{y}$ & $\mathrm{n}$ & 864.999 & -414.457 \\
\hline 4 & $\mathrm{n}$ & $\mathrm{n}$ & $\mathrm{y}$ & $\mathrm{y}$ & $\mathrm{y}$ & $\mathrm{y}$ & 865.9124 & -418.912 \\
\hline 3 & $\mathrm{n}$ & $\mathrm{n}$ & $\mathrm{y}$ & $\mathrm{y}$ & $\mathrm{n}$ & $\mathrm{y}$ & 865.9375 & -416.792 \\
\hline 3 & $\mathrm{y}$ & $\mathrm{n}$ & $\mathrm{n}$ & $\mathrm{y}$ & $\mathrm{n}$ & $\mathrm{y}$ & 866.2757 & -418.515 \\
\hline 3 & $\mathrm{n}$ & $\mathrm{n}$ & $\mathrm{y}$ & $\mathrm{n}$ & $\mathrm{y}$ & $\mathrm{y}$ & 866.4339 & -417.766 \\
\hline 2 & $\mathrm{n}$ & $\mathrm{n}$ & $\mathrm{n}$ & $\mathrm{y}$ & $\mathrm{n}$ & $\mathrm{y}$ & 866.575 & -418.722 \\
\hline 4 & $\mathrm{y}$ & $\mathrm{y}$ & $\mathrm{n}$ & $\mathrm{y}$ & $\mathrm{y}$ & $\mathrm{n}$ & 867.0663 & -417.182 \\
\hline 3 & $\mathrm{n}$ & $\mathrm{y}$ & $\mathrm{n}$ & $\mathrm{y}$ & $\mathrm{y}$ & $\mathrm{n}$ & 867.561 & -416.125 \\
\hline 2 & $\mathrm{n}$ & $\mathrm{y}$ & $\mathrm{n}$ & $\mathrm{n}$ & $\mathrm{y}$ & $\mathrm{n}$ & 867.5755 & -416.161 \\
\hline 3 & $\mathrm{y}$ & $\mathrm{y}$ & $\mathrm{n}$ & $\mathrm{n}$ & $\mathrm{y}$ & $\mathrm{n}$ & 867.5755 & -416.161 \\
\hline 2 & $\mathrm{n}$ & $\mathrm{n}$ & $\mathrm{n}$ & $\mathrm{y}$ & $\mathrm{y}$ & $\mathrm{n}$ & 867.826 & -408.325 \\
\hline 2 & $\mathrm{n}$ & $\mathrm{y}$ & $\mathrm{n}$ & $\mathrm{y}$ & $\mathrm{n}$ & $\mathrm{n}$ & 867.856 & -423.042 \\
\hline 3 & $\mathrm{y}$ & $\mathrm{n}$ & $\mathrm{n}$ & $\mathrm{y}$ & $\mathrm{y}$ & $\mathrm{n}$ & 868.1056 & -408.745 \\
\hline 2 & $\mathrm{n}$ & $\mathrm{y}$ & $\mathrm{n}$ & $\mathrm{n}$ & $\mathrm{n}$ & $\mathrm{y}$ & 868.3033 & -422.448 \\
\hline 4 & $\mathrm{y}$ & $\mathrm{n}$ & $\mathrm{y}$ & $\mathrm{y}$ & $\mathrm{y}$ & $\mathrm{n}$ & 868.4045 & -409.714 \\
\hline 3 & $\mathrm{y}$ & $\mathrm{y}$ & $\mathrm{n}$ & $\mathrm{y}$ & $\mathrm{n}$ & $\mathrm{n}$ & 869.0338 & -422.328 \\
\hline 2 & $\mathrm{n}$ & $\mathrm{n}$ & y & $\mathrm{n}$ & $\mathrm{n}$ & $\mathrm{y}$ & 869.2231 & -424.396 \\
\hline 1 & $\mathrm{n}$ & $\mathrm{n}$ & $\mathrm{n}$ & $\mathrm{n}$ & $\mathrm{n}$ & $\mathrm{y}$ & 869.2234 & -424.397 \\
\hline 2 & $\mathrm{n}$ & $\mathrm{n}$ & $\mathrm{n}$ & $\mathrm{n}$ & $\mathrm{y}$ & $\mathrm{y}$ & 869.6256 & -416.685 \\
\hline 2 & $\mathrm{y}$ & $\mathrm{y}$ & $\mathrm{n}$ & $\mathrm{n}$ & $\mathrm{n}$ & $\mathrm{n}$ & 869.9012 & -422.897 \\
\hline
\end{tabular}




\begin{tabular}{|c|c|c|c|c|c|c|c|c|}
\hline No. of factors used & $\begin{array}{l}\text { 훙 } \\
\text { ㅁ }\end{array}$ & 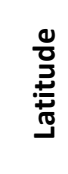 & 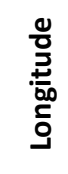 & 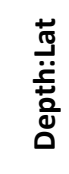 & 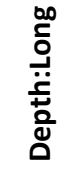 & 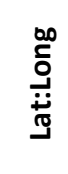 & AIC & Likelihood \\
\hline 2 & $\mathrm{n}$ & $\mathrm{y}$ & $\mathrm{y}$ & $\mathrm{n}$ & $\mathrm{n}$ & $\mathrm{n}$ & 870.9592 & -423.869 \\
\hline 3 & $\mathrm{n}$ & $\mathrm{y}$ & $\mathrm{y}$ & $\mathrm{n}$ & $\mathrm{n}$ & $\mathrm{y}$ & 870.9592 & -423.869 \\
\hline 1 & $\mathrm{n}$ & $\mathrm{y}$ & $\mathrm{n}$ & $\mathrm{n}$ & $\mathrm{n}$ & $\mathrm{n}$ & 872.3716 & -426.478 \\
\hline 3 & $\mathrm{y}$ & $\mathrm{n}$ & $\mathrm{y}$ & $\mathrm{y}$ & $\mathrm{n}$ & $\mathrm{n}$ & 875.583 & -428.89 \\
\hline 2 & $\mathrm{n}$ & $\mathrm{n}$ & $\mathrm{y}$ & $\mathrm{y}$ & $\mathrm{n}$ & $\mathrm{n}$ & 877.155 & -427.108 \\
\hline 3 & $\mathrm{n}$ & $\mathrm{n}$ & $\mathrm{y}$ & $\mathrm{y}$ & $\mathrm{y}$ & $\mathrm{n}$ & 878.3865 & -429.726 \\
\hline 2 & $\mathrm{y}$ & $\mathrm{n}$ & $\mathrm{y}$ & $\mathrm{n}$ & $\mathrm{n}$ & $\mathrm{n}$ & 879.0126 & -432.136 \\
\hline 3 & $\mathrm{y}$ & $\mathrm{n}$ & $\mathrm{y}$ & $\mathrm{n}$ & $\mathrm{y}$ & $\mathrm{n}$ & 880.4002 & -431.902 \\
\hline 2 & $\mathrm{n}$ & $\mathrm{n}$ & $\mathrm{y}$ & $\mathrm{n}$ & $\mathrm{y}$ & $\mathrm{n}$ & 881.0871 & -432.137 \\
\hline 2 & $\mathrm{y}$ & $\mathrm{n}$ & $\mathrm{n}$ & $\mathrm{n}$ & $\mathrm{y}$ & $\mathrm{n}$ & 881.2075 & -431.93 \\
\hline 1 & $\mathrm{n}$ & $\mathrm{n}$ & $\mathrm{n}$ & $\mathrm{y}$ & $\mathrm{n}$ & $\mathrm{n}$ & 881.411 & -431.709 \\
\hline 1 & $\mathrm{n}$ & $\mathrm{n}$ & $\mathrm{n}$ & $\mathrm{n}$ & $\mathrm{y}$ & $\mathrm{n}$ & 881.8953 & -431.269 \\
\hline 2 & $\mathrm{y}$ & $\mathrm{n}$ & $\mathrm{n}$ & $\mathrm{y}$ & $\mathrm{n}$ & $\mathrm{n}$ & 882.0745 & -435.929 \\
\hline 1 & $\mathrm{n}$ & $\mathrm{n}$ & $\mathrm{y}$ & $\mathrm{n}$ & $\mathrm{n}$ & $\mathrm{n}$ & 884.1311 & -437.479 \\
\hline 1 & $\mathrm{y}$ & $\mathrm{n}$ & $\mathrm{n}$ & $\mathrm{n}$ & $\mathrm{n}$ & $\mathrm{n}$ & 890.4032 & -441.121 \\
\hline
\end{tabular}

Table 2.7 VarTD models for $50 \mathrm{~m}$ or shallower sorted in ascending order of AIC (lowest AIC is the best model)

\begin{tabular}{|c|c|c|c|l|l|}
\hline $\begin{array}{l}\text { No. of factors } \\
\text { used }\end{array}$ & \multicolumn{4}{|c|}{ Latitude } & \multicolumn{2}{l|}{ Longitude } & Lat:Long & AIC & Likelihood \\
\hline 2 & $\mathrm{y}$ & $\mathrm{n}$ & $\mathrm{y}$ & 652.6805 & -315.5374 \\
\hline 1 & $\mathrm{n}$ & $\mathrm{n}$ & $\mathrm{y}$ & 653.0152 & -316.5345 \\
\hline 2 & $\mathrm{y}$ & $\mathrm{y}$ & $\mathrm{n}$ & 654.7527 & -316.2822 \\
\hline 1 & $\mathrm{y}$ & $\mathrm{n}$ & $\mathrm{n}$ & 655.2065 & -318.2103 \\
\hline 3 & $\mathrm{y}$ & $\mathrm{y}$ & $\mathrm{y}$ & 655.9727 & -317.9016 \\
\hline 2 & $\mathrm{n}$ & $\mathrm{y}$ & $\mathrm{y}$ & 657.6563 & -322.0735 \\
\hline 1 & $\mathrm{n}$ & $\mathrm{y}$ & $\mathrm{n}$ & 663.8724 & -327.2646 \\
\hline
\end{tabular}


Table 2.8 No. of genera recorded and AvTD and VarTD values for the DOC Bioregions DOC (and other areas within the EEZ)

\begin{tabular}{|c|c|c|c|c|c|c|}
\hline DOC Bioregion & $\begin{array}{c}\text { No. of } \\
\text { genera } \\
(0-200 \mathrm{~m})\end{array}$ & $\begin{array}{l}\text { No. of } \\
\text { genera } \\
(0-50 \mathrm{~m})\end{array}$ & $\begin{array}{c}\text { AvTD } \\
(0- \\
200 \mathrm{~m}) \\
\end{array}$ & $\begin{array}{c}\text { AvTD } \\
(0- \\
50 \mathrm{~m})\end{array}$ & $\begin{array}{c}\text { VarTD } \\
(0- \\
200 \mathrm{~m})\end{array}$ & $\begin{array}{c}\text { VarTD } \\
(0- \\
50 \mathrm{~m}) \\
\end{array}$ \\
\hline Three Kings Islands & 84 & 55 & 63.06 & 60.77 & 201.52 & 160.22 \\
\hline Northeastern & 160 & 117 & 64.65 & 63.92 & 191.36 & 182.13 \\
\hline Western North Island & 52 & 52 & 62.02 & 62.02 & 163.91 & 163.91 \\
\hline Eastern North Island & 36 & 31 & 64.11 & 64.92 & 189.13 & 197.78 \\
\hline North Cook Strait & 74 & 73 & 66.36 & 66.5 & 221.47 & 220.48 \\
\hline South Cook Strait & 38 & 38 & 62.87 & 62.87 & 189.45 & 189.45 \\
\hline West Coast South Island & 4 & 4 & $\mathrm{~N} / \mathrm{A}$ & $\mathrm{N} / \mathrm{A}$ & $\mathrm{N} / \mathrm{A}$ & $\mathrm{N} / \mathrm{A}$ \\
\hline East Coast South Island & 37 & 35 & 65.3 & 65.78 & 202.62 & 200.39 \\
\hline Southern & 41 & 40 & 64.88 & 65.03 & 223.77 & 222.76 \\
\hline Fiordland & 20 & 20 & 65.99 & 65.99 & 217.28 & 217.28 \\
\hline Snares Islands & 0 & 0 & $\mathrm{~N} / \mathrm{A}$ & $\mathrm{N} / \mathrm{A}$ & $\mathrm{N} / \mathrm{A}$ & $\mathrm{N} / \mathrm{A}$ \\
\hline Chatham Islands & 64 & 23 & 61.58 & 58.75 & 166.67 & 104.48 \\
\hline Auckland Islands & 23 & 16 & 57.71 & 56.98 & 109.81 & 124.47 \\
\hline Campbell Islands & 8 & 6 & $\mathrm{~N} / \mathrm{A}$ & $\mathrm{N} / \mathrm{A}$ & $\mathrm{N} / \mathrm{A}$ & $\mathrm{N} / \mathrm{A}$ \\
\hline Antipodes Islands & 7 & 7 & $\mathrm{~N} / \mathrm{A}$ & $\mathrm{N} / \mathrm{A}$ & $\mathrm{N} / \mathrm{A}$ & $\mathrm{N} / \mathrm{A}$ \\
\hline Bounty Islands & 8 & 8 & $\mathrm{~N} / \mathrm{A}$ & $\mathrm{N} / \mathrm{A}$ & $\mathrm{N} / \mathrm{A}$ & $\mathrm{N} / \mathrm{A}$ \\
\hline Campbell Plateau & 7 & 4 & $\mathrm{~N} / \mathrm{A}$ & $\mathrm{N} / \mathrm{A}$ & $\mathrm{N} / \mathrm{A}$ & $\mathrm{N} / \mathrm{A}$ \\
\hline Chatham Rise & 31 & 4 & 57.53 & $\mathrm{~N} / \mathrm{A}$ & 140.25 & $\mathrm{~N} / \mathrm{A}$ \\
\hline Kermadec Islands & 2 & 2 & $\mathrm{~N} / \mathrm{A}$ & $\mathrm{N} / \mathrm{A}$ & $N / A$ & $\mathrm{~N} / \mathrm{A}$ \\
\hline
\end{tabular}

Table 2.9 No. of genera recorded and AvTD and VarTD values for the Shears Bioregions.

\begin{tabular}{|c|c|c|c|c|c|c|}
\hline Shears Bioregion & $\begin{array}{l}\text { No. of genera } \\
(0-200 \mathrm{~m})\end{array}$ & $\begin{array}{l}\text { No. of genera } \\
(0-50 \mathrm{~m})\end{array}$ & $\begin{array}{l}\text { AvTD (0- } \\
200 \mathrm{~m})\end{array}$ & $\begin{array}{l}\text { AvTD (0- } \\
50 \mathrm{~m})\end{array}$ & $\begin{array}{c}\text { VarTD } \\
(0-200 \mathrm{~m})\end{array}$ & $\begin{array}{c}\text { VarTD } \\
(0-50 \mathrm{~m})\end{array}$ \\
\hline Three Kings & 84 & 55 & 63.06 & 60.77 & 201.52 & 160.22 \\
\hline Northeastern & 160 & 117 & 64.65 & 63.92 & 191.36 & 182.13 \\
\hline Abel & 60 & 60 & 62.99 & 62.99 & 173.31 & 173.31 \\
\hline Portland & 36 & 31 & 64.11 & 64.92 & 189.13 & 197.78 \\
\hline Cook & 59 & 58 & 68.09 & 68.31 & 227.66 & 224.7 \\
\hline Raglan & 52 & 52 & 62.02 & 62.02 & 163.91 & 163.91 \\
\hline Buller & 4 & 4 & $\mathrm{~N} / \mathrm{A}$ & N/A & $\mathrm{N} / \mathrm{A}$ & N/A \\
\hline Banks & 26 & 20 & 62.08 & 63.55 & 145.49 & 151.85 \\
\hline Chalmers & 29 & 27 & 62.16 & 62.43 & 188.08 & 194.97 \\
\hline Stewart Island & 30 & 30 & 67.36 & 67.36 & 231.8 & 231.8 \\
\hline Fiordland & 20 & 20 & 65.99 & 65.99 & 217.28 & 217.28 \\
\hline
\end{tabular}


Chapter 2 Spatial patterns of sponge biodiversity in New Zealand coastal waters 


\section{CHAPTER 3}

SPATIAL VARIABILITY OF SPONGE ASSEMBLAGES ON THE WELLINGTON SOUTH COAST, NEW ZEALAND. 


\subsection{ABSTRACT}

The aim of this study was to provide baseline data for sponge biodiversity on the Wellington south coast, New Zealand. Eighteen sites were qualitatively sampled resulting in the identification of 65 different sponge taxa. Forty-three of these species were also reported from a quantitative survey of three vertical wall sites at 8-10 m depth conducted as part of a long-term monitoring study. All three sites had a similar mean number of species per quadrat, however, the percentage cover of sponges and sponge density varied significantly between sites. ANOSIM showed that all three sites were significantly different from each other $(\mathrm{P}<0.001)$, which is likely the result of differences in environmental conditions. The Wellington south coast has a diverse sponge assemblage, which is atypically dominated by calcareous species of the genus Clathrina. This appears to be unusual for New Zealand, and although the reasons for this situation are unknown, a potential hypothesis is that this situation is explained by the low levels of nutrients on the Wellington south coast, allowing calcareous sponges to proliferate at the expense of the demosponges. 


\subsection{INTRODUCTION}

The distribution of the sponge fauna of New Zealand was discussed in the second chapter of this thesis. That chapter provided an overview of national sponge biodiversity, however, it did not take into account the spatial and temporal variability of sponge biodiversity. This chapter will focus on the sponge assemblage spatial diversity patterns within the Wellington region, in part because the Cook bioregion has high values of AvTD and VarTD.

Spatial diversity describes how organisms are distributed at different scales. There have been many studies on sponges describing spatial variability in genetic structure (Solé-Cava et al., 1991; Boury-Esnault et al., 1992; Solé-Cava et al., 1992; Solé-Cava \& Boury-Esnault, 1999; Miller et al., 2001; Wörheide et al., 2008); biochemical composition (Page et al., 2005; Abdo et al., 2007); morphology (Meroz-Fine et al., 2005); and distribution patterns of specific species (Boury-Esnault et al., 1993; Kelly, 2003; Usher et al., 2004). Spatial variation in abundance and diversity have also been reported at different levels: within a particular habitat - alpha diversity (Barnes, 1999; Bell, 2007a; Carballo \& Nava, 2007; Wulff, 2008); variation within geographic areas - beta diversity (Adjeroud \& Salvat, 1996; Hooper et al., 2002; Kefalas et al., 2003; Bell \& Smith, 2004; Duckworth et al., 2008); and variability across different geographic regions - gamma diversity (Wilkinson, 1987; Wilkinson \& Cheshire, 1990; Wulff, 1996). Variation in sponge diversity, distribution and abundance is likely to influence other benthic organisms due to the important functional roles that sponges fulfill. (Pang, 1973; Rützler, 1975; Wulff \& Buss, 1979; Corredor et al., 1988; Ribeiro et al., 2003; Bell, 2007a; Bell \& Carballo, 2008; Bell, 2008). Sponge assemblages are influenced by a number of physical factors including: depth (Wilkinson \& Evans, 1989; Duckworth et al., 2004; Cleary et al., 2005); water flow (Leichter \& Witman, 1997; Duckworth et al., 2004); temperature (Riisgard et al., 1993; Barnes, 1999); light intensity (Alcolado, 1994); sedimentation (Sara \& Vacelet, 1973; Wilkinson \& Vacelet, 1979; Zea, 1994; Ilan \& Abelson, 1995) and salinity (Fell et al., 1989; Leamon \& Fell, 1990; Barnes, 1999). Biological factors influencing sponge assemblages include: predation 
(Wulff, 1994, 1995b, 1997b, 2000); mutualistic and symbiotic associations (Rosell \& Uriz, 1992; Arillo et al., 1993; Wulff, 1997a); concentration and diversity of planktontonic food resources (Pile, 1996; Ribes et al., 1999; Ribes et al., 2003); spatial competition (Jackson, 1977; Aerts, 1998; Fagerstrom et al., 2000); and disease (Smith, 1941; Galtsoff, 1942; Pronzato, 1999; Wulff, 2007). There has been some discussion regarding the relative roles of biological and physical factors in controlling sponge assemblages in different geographic regions. Biological factors may be more important than physical factors in determining tropical sponge assemblage structure in the Caribbean, however, physical factors have been found to be most important in the NE Atlantic and Eastern Pacific (Wulff, 1997a; Bell \& Barnes, 2000a; Wulff, 2000; Bell \& Barnes, 2003a; Carballo, 2006).

Some of the sponge species and typical sponge habitats on the Wellington south coast (WSC) have recently been described, although work focused on relatively few common species from only a few sites (Duckworth \& Battershill, 2001; Duckworth et al., 2004; Berman et al., 2008). The aim of this study was to provide baseline data for the sponge biodiversity of the Wellington south coast, and use this information to identify suitable sites for permanent quantitative quadrats that are representative of sponge biodiversity and abundance on the Wellington south coast; these sites will be used to investigate temporal variation in sponge assemblages in chapter four. 


\subsection{Methods}

\subsubsection{STUDY AREA}

Sponge assemblages were qualitatively sampled at eighteen sites along the south coast of Wellington, New Zealand ( $41^{\circ} 21^{\prime} \mathrm{S}, 174^{\circ} 45^{\prime} \mathrm{E}$ ) (Fig. 3.1). Three sites were chosen from the eighteen sites for quantitative analysis after the preliminary biodiversity survey. These were: Near Sirens (4120'56.43"S, $174^{\circ} 45^{\prime} 52.46^{\prime E}$ ); Far Sirens (4120'58.89"S, 174²4'54.26"E); and Breaker Bay

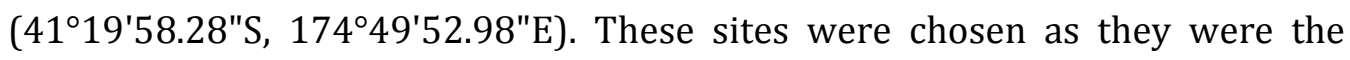
most easily accessible sponge-dominated sites with continuous similar vertical bedrock habitat at a similar depth $(8-10 \mathrm{~m})$ along the Wellington south coast (WSC).

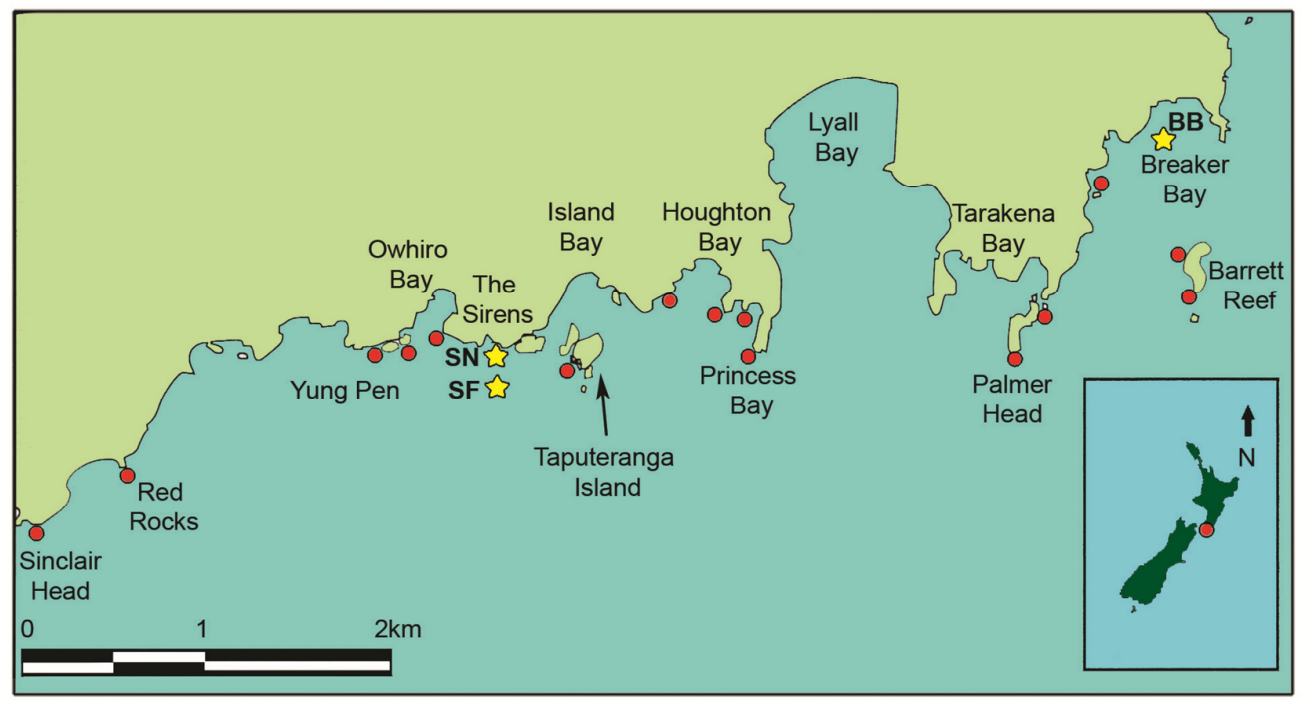

Fig. 3.1 Surveyed sites along the Wellington south coast, New Zealand. The red circles mark the positions of the fifteen sites surveyed for biodiversity. The yellow stars mark the three sites surveyed for biodiversity and chosen for quantitative analysis of the sponge assemblages along the Wellington south coast, New Zealand. These sites are: SN $\rightarrow$ Near Sirens; SF $\rightarrow$ Far Sirens; and BB $\rightarrow$ Breaker Bay. 
The study area has a south to south-east aspect, and is exposed to frequent high-energy south-easterly swells and storm episodes interspersed by northwesterly winds from which the shores are afforded relative protection by high cliffs. There are three separate water bodies that combine to form the Cook Strait current that flows past the study sites: the Southland Current (a mix of subtropical and sub-Antarctic water) that flows northward up the east coast of the South Island and westward into the Cook Strait, the East Cape Current (subtropical water) that flows south along the east coast of the North Island and then westwards into Cook Strait and the D'Urville Current (warm subtropical water) that flows from north to south from the Tasman sea (Heath, 1971). Tidal currents in the region can reach speeds of up to $360 \mathrm{cms}^{-1}$ (Carter, 2008). Maximum wave heights can exceed $13 \mathrm{~m}$ during large storm events, while wave height (the highest one-third of the waves) can exceed $8 \mathrm{~m}$ (Carter et al., 2002). These storm events inevitably influences intertidal and subtidal community structure. The monthly mean sea temperature $( \pm$ SE) between May 2008 and May 2009 was $15.67^{\circ} \mathrm{C} \pm 0.03$.

\subsubsection{BIODIVERSITY SURVEY}

Specimens were collected by SCUBA during a thorough search of the sites including underneath rocks and overhangs along $12 \mathrm{~km}$ of the WSC and at a depth of up to $18 \mathrm{~m}$ between September 2007 and April 2009. Small specimens (approximately $1 \mathrm{~cm}^{3}$ ) were collected from each sponge. Photographs of the sponges were taken in situ using an Olympus 5060 camera on the macro setting. Tissue preparations were prepared by sectioning. Sections were taken horizontally from the surface of the sponge and also vertically at a $90^{\circ}$ angle through the specimen. The sections were dehydrated in absolute alcohol and then placed in Histoclear to clarify the tissue before being mounted on a microscopic slide using DePeX mounting medium. Spicule preparations were prepared directly on microscope slides by dissolving a small tissue sample (1 $\mathrm{mm}^{3}$ ) in a few drops of concentrated nitric acid, which was gently heated by repeatedly pulling the slide across the flame of a Bunsen burner in a fume 
cupboard. Examples of some of the spicule preparations under a microscope are shown in Fig. 3.2. Additional drops of acid were added to replace evaporated acid until the remaining spicules were clean. The slide was then rinsed with a few drops of absolute ethanol, dried and mounted using DePeX mounting medium.

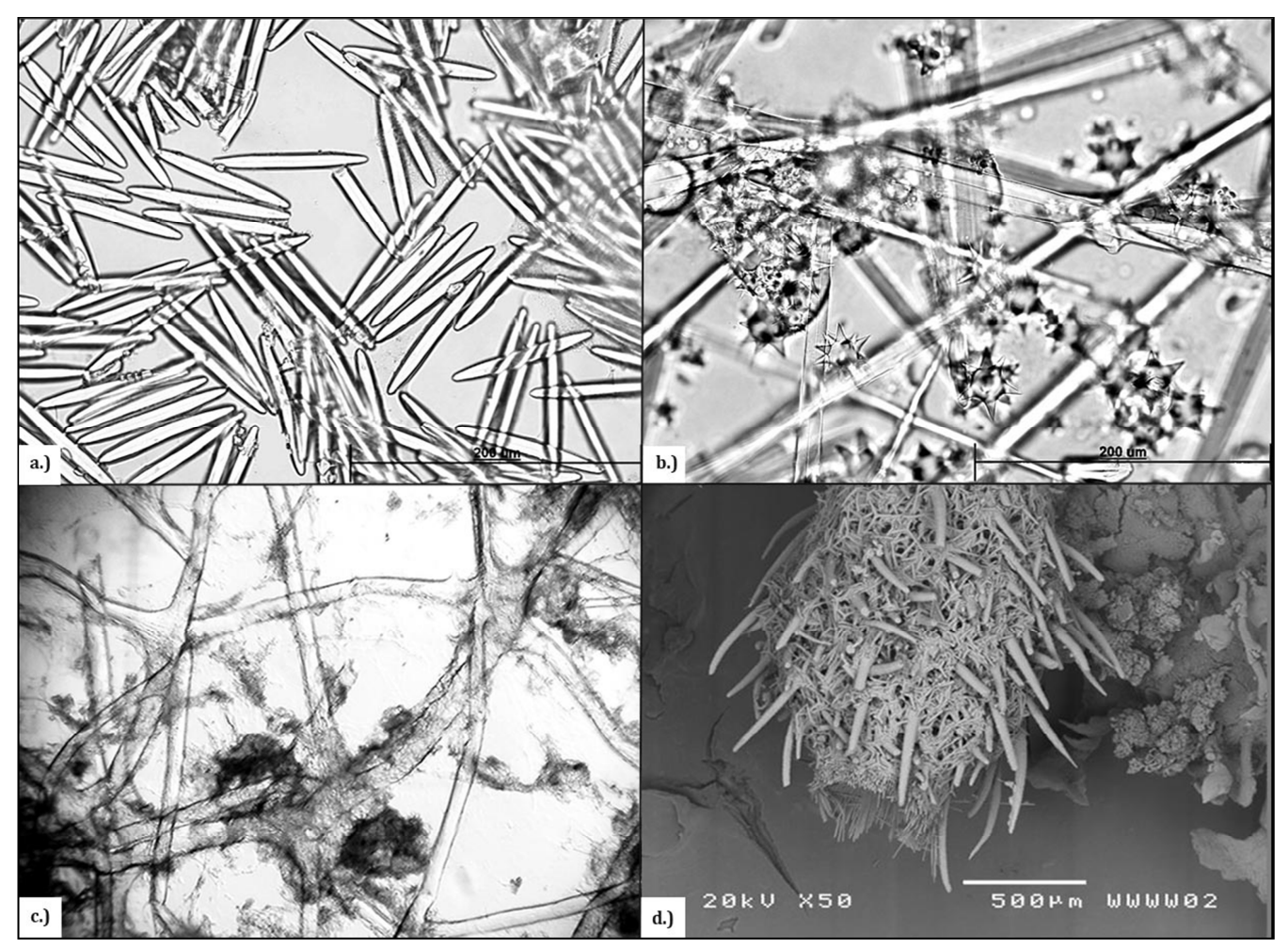

Fig. 3.2 Spicules and spongin fibres from sponges of the Wellington south coast. a) Oxea spicules from Haliclona c.f. venustina (Bergquist, 1961) b) Aster microsclere spicules from Tethya berquistae (Hooper, 1994) c) 20x maginification pf Spongin fibres from Dendrilla rosa (Lendenfeld, 1883) d) SEM of Sycon sp. Depicting the skeletal arrangement of the calcareous triactine and diactine spicules. Pictures a-c taken by the author, picture $d$ taken by Michelle Carter. 


\subsubsection{SPATial SURVEY}

Six $0.5 \mathrm{~m}^{2}$ haphazardly placed quadrats (within the available vertical bedrock) were taken at three of the sites used for quantitative analyses (see above) in April 2008. All sponges were surveyed in the same month to prevent temporal variation affecting the sponge assemblage differences. Each quadrat was divided into $25 \times 10 \mathrm{~cm}^{2}$ sections and each section was photographed. Within each section the number of patches and the percentage cover of each species were recorded from the pictures. If a sponge overlapped two sections the patch was only counted once, however, the percentage cover was recorded for all sections it covered. Percentage cover was calculated using an overlaying grid of 100 dots for each separate photograph. The sponge density, percentage cover, mean species richness and Shannon index were calculated for each site.

The statistical package Plymouth Routines in Multivariate Ecological Research [PRIMER 6] was used to analyse the sponge assemblage data. Species accumulation curves in PRIMER were plotted for each site using UGE from DIVERSE (Ugland et al., 2003; Clarke \& Gorley, 2006). The abundance data from each site was pre-treated using a dispersion factor to down-weigh the impact of clumping of highly abundant species in relation to more evenly dispersed species. The three conditions that have to be met in order to use a dispersion factor are: that the data for each species are genuine counts not densities that have been standardised; replicates need to be independent within the sample groups; and that each replicate is of uniform size. The percentage cover data was $\log (\chi+1)$ transformed to down-weigh the influence of highly abundant species.

'Site' and 'Quadrat' were considered as a priori factors for the data analysis. A similarity matrix was then created using a zero adjusted Bray-Curtis similarity analysis to identify any similarities and differences between the sites and quadrats within sites. A dummy variable of one was used in a zero-adjusted Bray-Curtis analysis to prevent Bray-Curtis behaving erratically as values in some samples approached zero (Clarke et al., 2006; Rützler et al., 2007).

Ordination was carried out using non-Metric Multi-Dimensional Scaling (nMDS) 
to determine the relationships between the replicates at the different sites, and between sites. Clusters identified by the SIMPROF test elucidated any significant similarities between replicates without the bias of the a priori groupings at the site level and were presented using MDS plots.

ANOSIM was used to determine if any differences in assemblage structure existed between sites. Where ANOSIM R=1, the groups are completely different and where ANOSIM R=0, they are exactly the same. Permutation tests are used to determine if significant differences exist between sites. Finally, SIMPER analysis was used on the dispersion-weighted data, and the square root transformed percentage cover data, to elucidate which sponge species were contributing to the similarities between and within sites and which species characterised each site.

To investigate how well the multivariate pattern based on sponge abundances (densities) reflected the multivariate pattern of sponge percentage cover, the RELATE routine within PRIMER was used, which compared the matrices using a Spearman rank correlation test. The significance level of the test was determined by 1000 permutations under the null hypothesis of no relationship between the similarity matrices. 


\subsection{RESULTS}

\subsubsection{BIODIVERSITY SURVEY}

Sixty five sponge taxa were reported from the WSC from 27 families and eleven orders of demosponges and four families and three orders of calcareous sponges (Table 3.1). Of the species found, 38 have been identified to specieslevel with the remaining 26 identified to genus level, apart from one species that could only be identified to order (a Poecilisclerid). Some of the distinctive sponges are shown in Fig. 3.3. There were nine calcareous sponge species recorded, four species of Clathrina, two each of Leucetta and Leucosolenia and a Sycon species. The current status of the taxonomy of calcareous sponges combines many species together (Rapp, 2006; Clarke et al., 2008), however, the species listed here as distinct, differ in combinations of colour (in situ and also when air dried), external morphology, spicule complement and skeletal structure. Also they coexisted in the same habitat type of vertical bedrock and under overhangs, therefore they are distinct enough to be considered separate species (even for those only identified to genus level). 


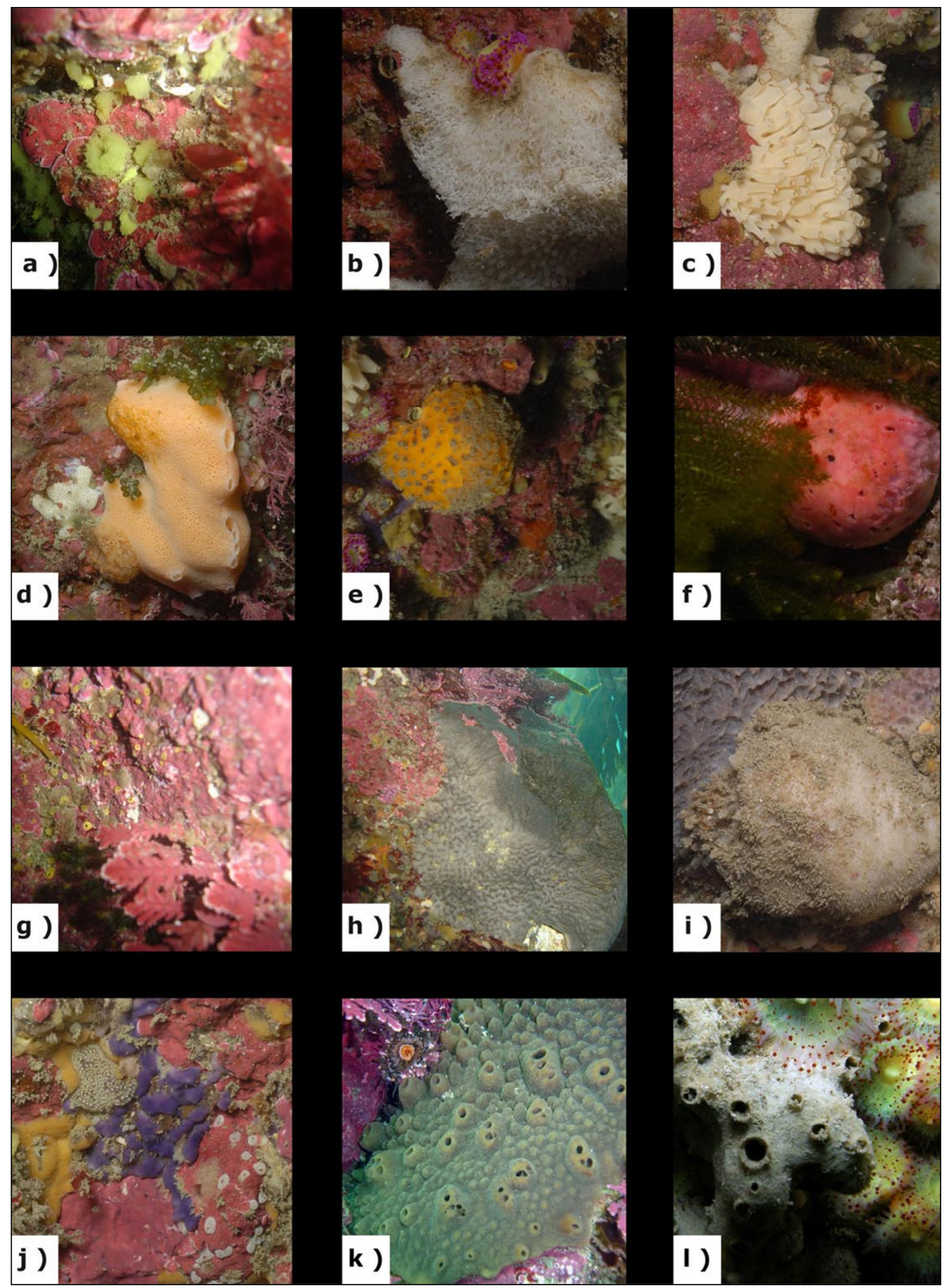

Fig. 3.3 Common sponge species from the Wellington south coast. a) Clathrina sp 1. yellow b) Clathrina sp. 2 white c) Leucosolenia echinata Kirk, 1894 d) Leucetta sp. e) Tethya burtoni Sarà \& Sarà, 2004 f) Tethya bergquistae Hooper, 1994 g) Cliona sp. h) Ancorina alata Dendy, 1924, i) Stelletta sp. j) Plakina c.f. trilopha Schulze, 1880 sensu Bergquist (1968) k) Latrunculia Biannulata wellingtonensis Alvarez et al., 2002 I) Chondropsis topsenti Dendy, 1895. 
Chapter 3 Spatial variability of sponge assemblages on the Wellington South Coast

Table 3.1 Sponge taxa listed in alphabetical order recorded from the Wellington south coast. Genus followed by sp. indicates that the sponge has been identified only to genus level. * indicates the taxa was also found during the quantitative survey Berman et al. (2008).

\begin{tabular}{|c|c|c|c|}
\hline Genus & Subgenus & Species & Authority \\
\hline Ancorina* & & alata & Dendy, 1924 \\
\hline Ancorina & c.f. & novaezelandiae & Dendy, 1924 \\
\hline Callyspongia* & & bathami & Bergquist \& Warne, 1980 \\
\hline Callyspongia & c.f. & ramosa & Gray, 1843 \\
\hline Callyspongia* & & sp. & Bergquist, 1961 \\
\hline Chelonaplysilla* & & violacea & Lendenfeld, 1883 \\
\hline Chondropsis & & $\mathrm{sp}$. & \\
\hline Chondropsis* & & topsenti & Dendy, 1895 \\
\hline Cinachyra* & & sp. & \\
\hline Clathria* & & sp. & \\
\hline Clathrina* & & sp. 1 & \\
\hline Clathrina* & & sp. 2 & \\
\hline Clathrina* & & sp. 3 & \\
\hline Clathrina* & & sp. 4 & \\
\hline Cliona* & & sp. & \\
\hline Crella & Pytheas & incrustans & Carter, 1885 \\
\hline Crella* & & sp. & \\
\hline Darwinella* & & oxeata & Bergquist, 1961 \\
\hline Dendrilla * & & rosea & Lendenfeld, 1883 \\
\hline Dysidea * & & sp. 1 & \\
\hline Dysidea* & & sp. 2 & \\
\hline Halichondria* & Halichondria & moorei & Bergquist, 1961 \\
\hline Haliclona* & c.f. & venustina & Bergquist, 1961 \\
\hline Haliclona* & & sp. & \\
\hline Haliclona* & Haliclona & sp. & \\
\hline Halisarca & & dujardini & Johnston, 1842 \\
\hline Halisarca* & & sp. & \\
\hline Hamigera & & macrostrongyla & Bergquist \& Fromont, 1988 \\
\hline Hymeniacidon* & & perlevis & Montagu, 1818 \\
\hline lophon* & & $\mathrm{sp}$. & \\
\hline lophon & & minor & Brøndsted, 1924 \\
\hline lophon & & proximum & Ridley, 1881 \\
\hline Latrunculia & Biannulata & wellingtonensis & Alvarez et al., 2002 \\
\hline Leucetta* & & sp. 1 & \\
\hline Leucetta* & & sp. 2 & \\
\hline
\end{tabular}


Chapter 3 Spatial variability of sponge assemblages on the Wellington South Coast

\begin{tabular}{|c|c|c|c|}
\hline Genus & Subgenus & Species & Authority \\
\hline Leucosolenia* & & echinata & Kirk, 1893 \\
\hline Leucosolenia* & & sp. & \\
\hline Mycale* $^{*}$ & & sp. & \\
\hline Oscarella* & & lobularis & Schmidt, 1862 \\
\hline Oscarella* & & sp. & \\
\hline Plakina & & monolopha & Schulze, 1880 \\
\hline Plakina* & c.f. & trilopha & Schulze, 1880 \\
\hline Polymastia & c.f. & lorum & Kelly-Borges \& Bergquist, 1997 \\
\hline Polymastia* & & granulosa & Brøndsted, 1924 \\
\hline Polymastia & & hirsuta & Bergquist, 1968 \\
\hline Polymastia & c.f. & massilis & Carter, 1886 \\
\hline Polymastia & & fusca & Bergquist, 1961 \\
\hline Polymastia & c.f. & aurantium & Kelly-Borges \& Bergquist, 1997 \\
\hline Polymastia & & croceus & Kelly-Borges \& Bergquist, 1997 \\
\hline Psammocinia & & sp. & Cook \& Bergquist, 1996 \\
\hline Raspailia & Raspailia & topsenti & Dendy, 1924 \\
\hline Stelletta* & & sp. & \\
\hline Stelletta & c.f. & purpurea & Ridley, 1884 \\
\hline Stelletta & c.f. & arenaria & Bergquist, 1968 \\
\hline Strongylacidon* & & conulosa & Bergquist \& Fromont, 1988 \\
\hline Suberites & & cupuloides & Bergquist, 1961 \\
\hline Sycon * & & sp. & Grace \& Grace, 1976 \\
\hline Tedania * & & diversirhaphidophora & Brøndsted, 1923 \\
\hline Tethya* & & aurantium & Pallas, 1766 \\
\hline Tethya* & & bergquistae & Hooper, 1994 \\
\hline Tethya * & & burtoni & Sarà \& Sarà, 2004 \\
\hline Thorecta* $^{*}$ & & reticulata & Cook \& Bergquist, 1996 \\
\hline Thymosia & & sp. & \\
\hline \multicolumn{4}{|c|}{ Unidentified Poecilisclerid* } \\
\hline Xestospongia* & & sp. & Lawson et al., 1984 \\
\hline
\end{tabular}




\subsubsection{Spatial SURVeY}

Forty-three species of sponge were found across the three quantitative study sites. Results of species accumulation plots predicted that eighteen quadrats were sufficient to record the majority of species from the three sites combined and that the six quadrats were sufficient for each of the sites separately (Fig. 3.4 \& 3.5).

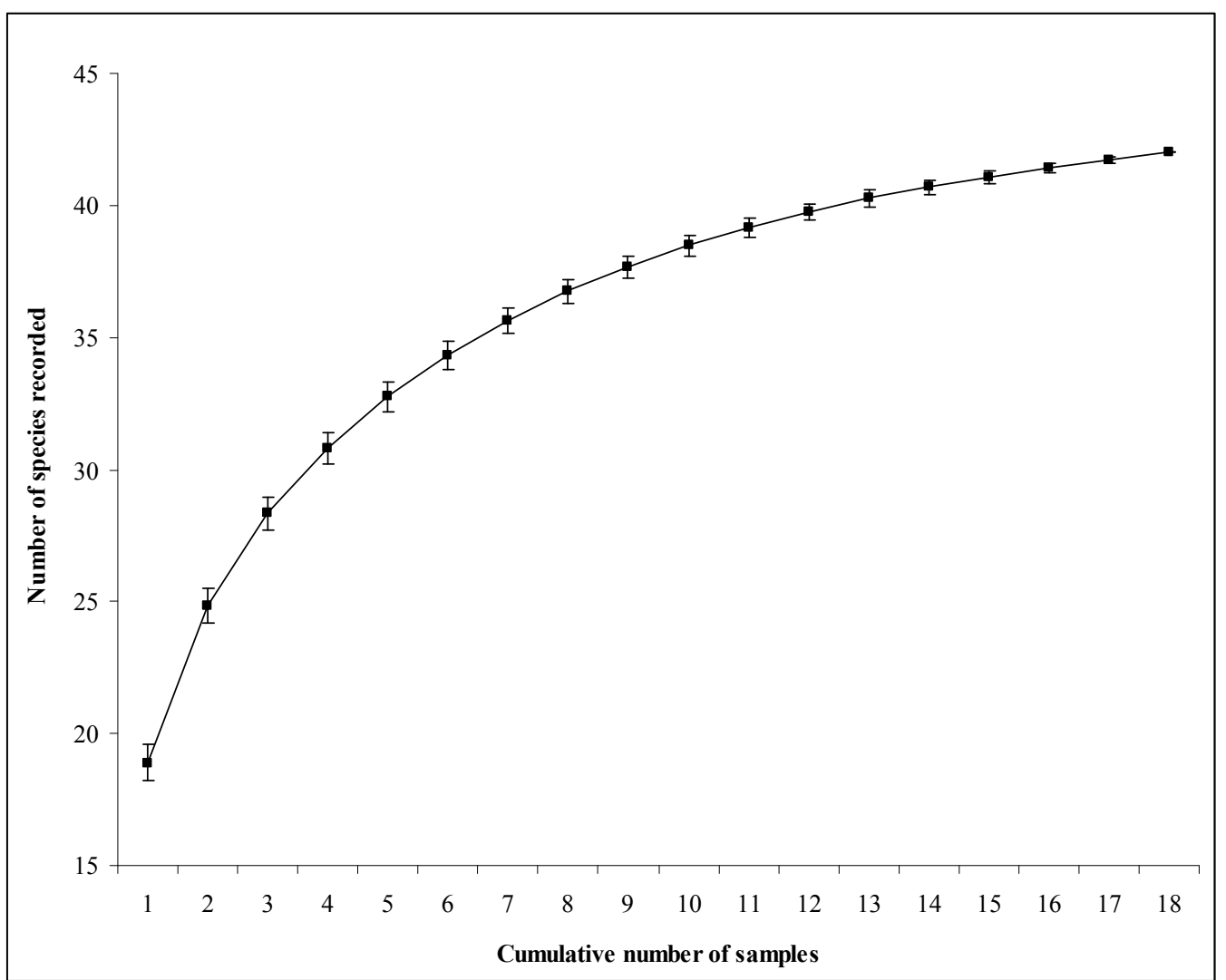

Fig. 3.4 Species accumulation curve Species accumulation curves (using the UGE method (Ugland et al., 2003)) for the Wellington south coast. The curve combines data from Breaker Bay, Far Sirens and Near Sirens replicates for the eighteen quadrats sampled across all three sites. 


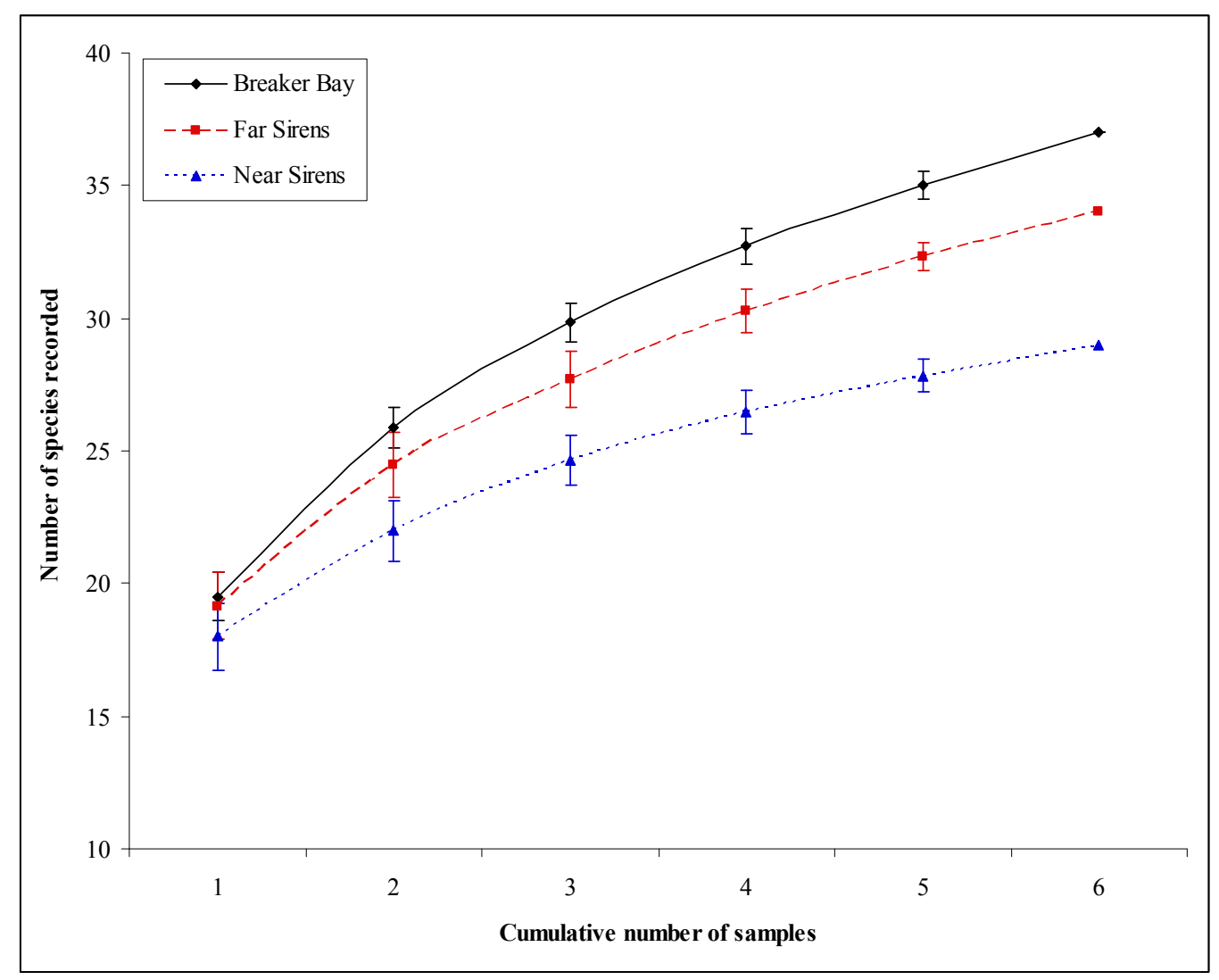

Fig. 3.5 Species accumulation curve (UGE method (Ugland et al., 2003)) comparing Breaker Bay, the Far Sirens and the Near Sirens sites.

All three sites had a similar mean number of species per quadrat ( $\pm \mathrm{SE}$ ); Breaker Bay $19.5 \pm 0.99$; Far Sirens $19.17 \pm 1.40$ and Near Sirens $18 \pm 1.37$. The Shannon diversity indices $(\mathrm{H})$ were also similar between sites; Breaker Bay 2.49 \pm 0.05 ; Far Sirens $2.34 \pm 0.09$ and Near Sirens $1.92 \pm 0.06$.

The calcareous sponges of the genus Clathrina were the most abundant sponges, particularly at the Near Sirens site. Oscarella sp. and Halisarca sp. were also abundant, in particular at both Sirens sites (Fig. 3.6.). Ancorina alata and Stelletta sp. dominated the sites in terms of percentage cover (Fig. 3.7), while Clathrina sp. Halisarca sp., Leucosolenia sp. and Plakina trilopha also had high percentage cover, particularly at the Near Sirens site. 


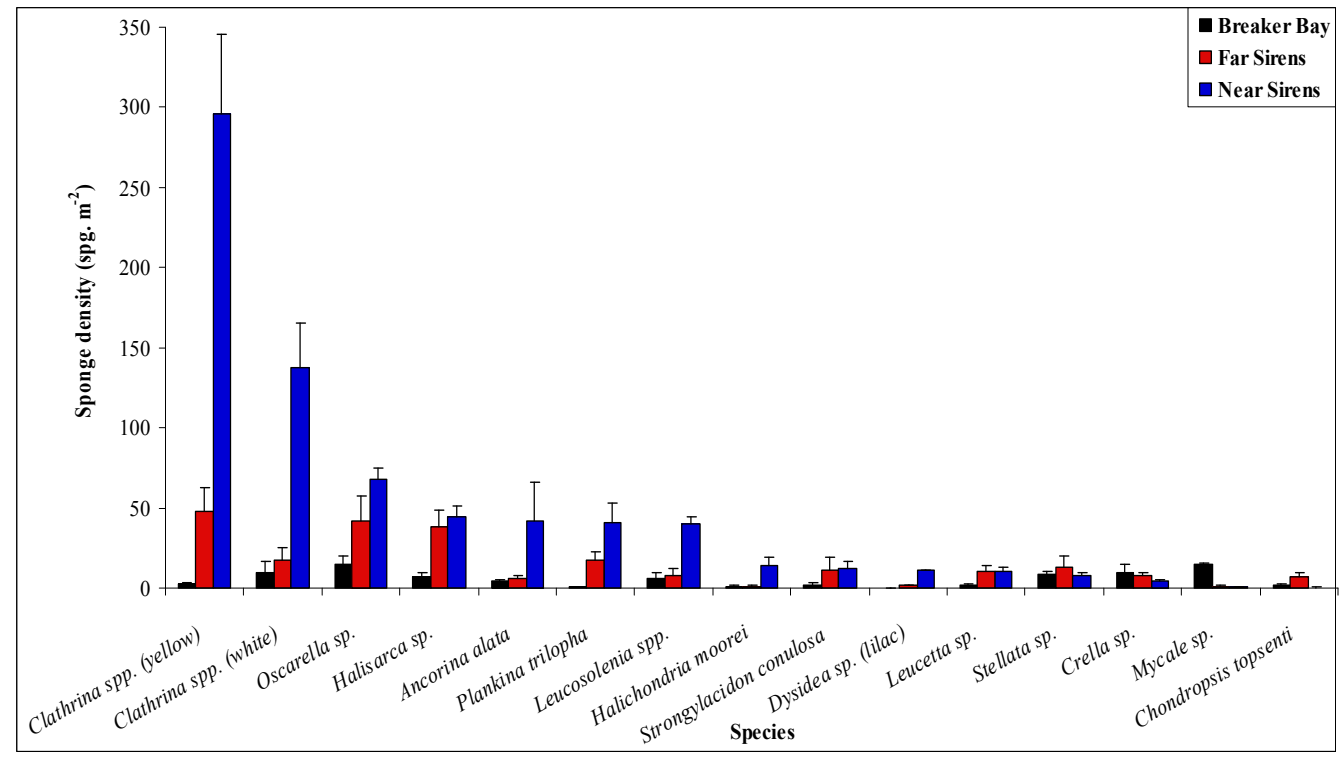

Fig. 3.6 Sponge density $\left(\mathrm{m}^{-2}\right)$ at Breaker Bay, the Far Sirens and the Near Sirens sites for the fifteen most abundant sponge species.

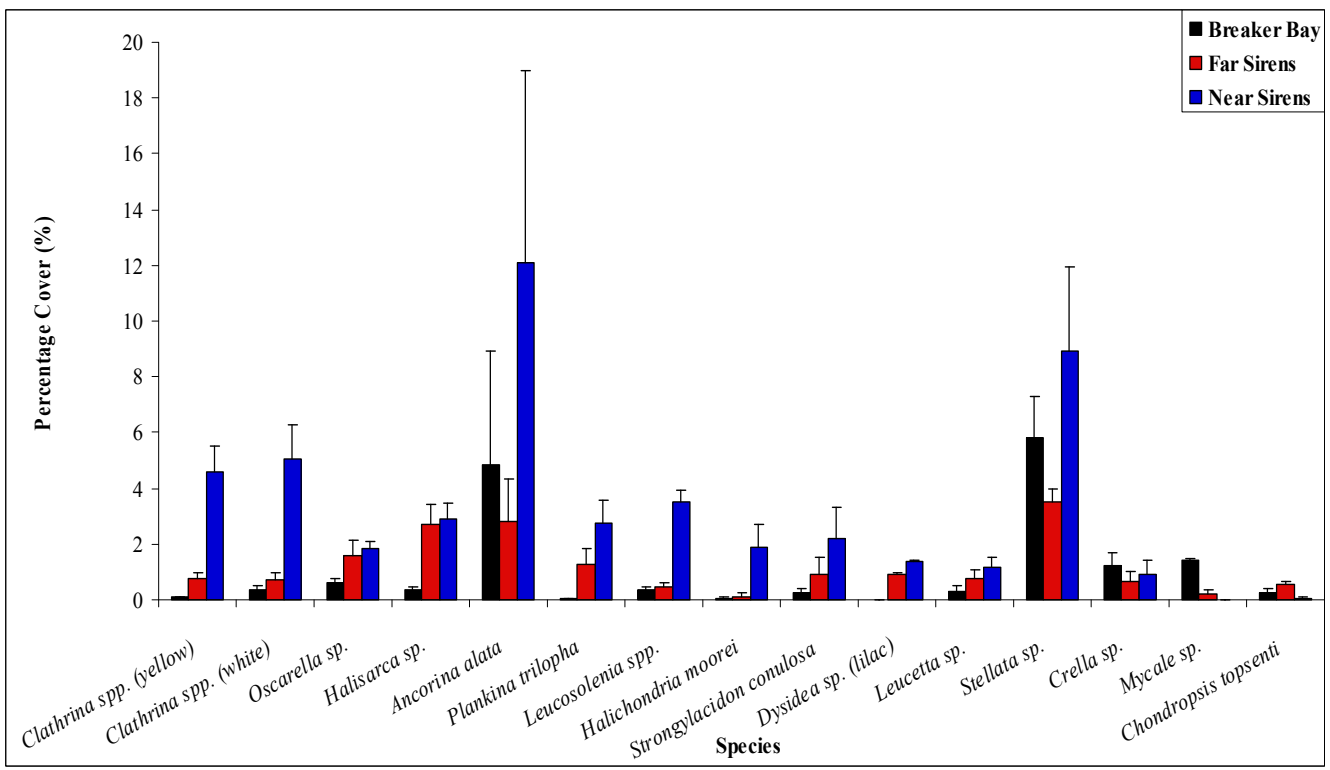

Fig. 3.7 Percentage Cover for the fifteen most abundant sponge species at Breaker Bay, the Far Sirens and the Near Sirens sites. 
The results of the MDS plots for percentage cover and density separates the sites, and significant $(\mathrm{P}<0.005)$ clusters were identified using SIMPROF. Clusters overlaid from the density data that had been down-weighted using a Dispersion Index indicated that the Near Sirens and the Far Sirens quadrats, formed a Sirens group, while the Breaker Bay replicates form a significantly differentiated cluster $(\mathrm{P}<0.05)$ (Fig. 3.8). Within the Sirens group the Near Sirens sites form a significantly different cluster and the Far Sirens form three significant groups, which cluster in turn with the Near Sirens group.

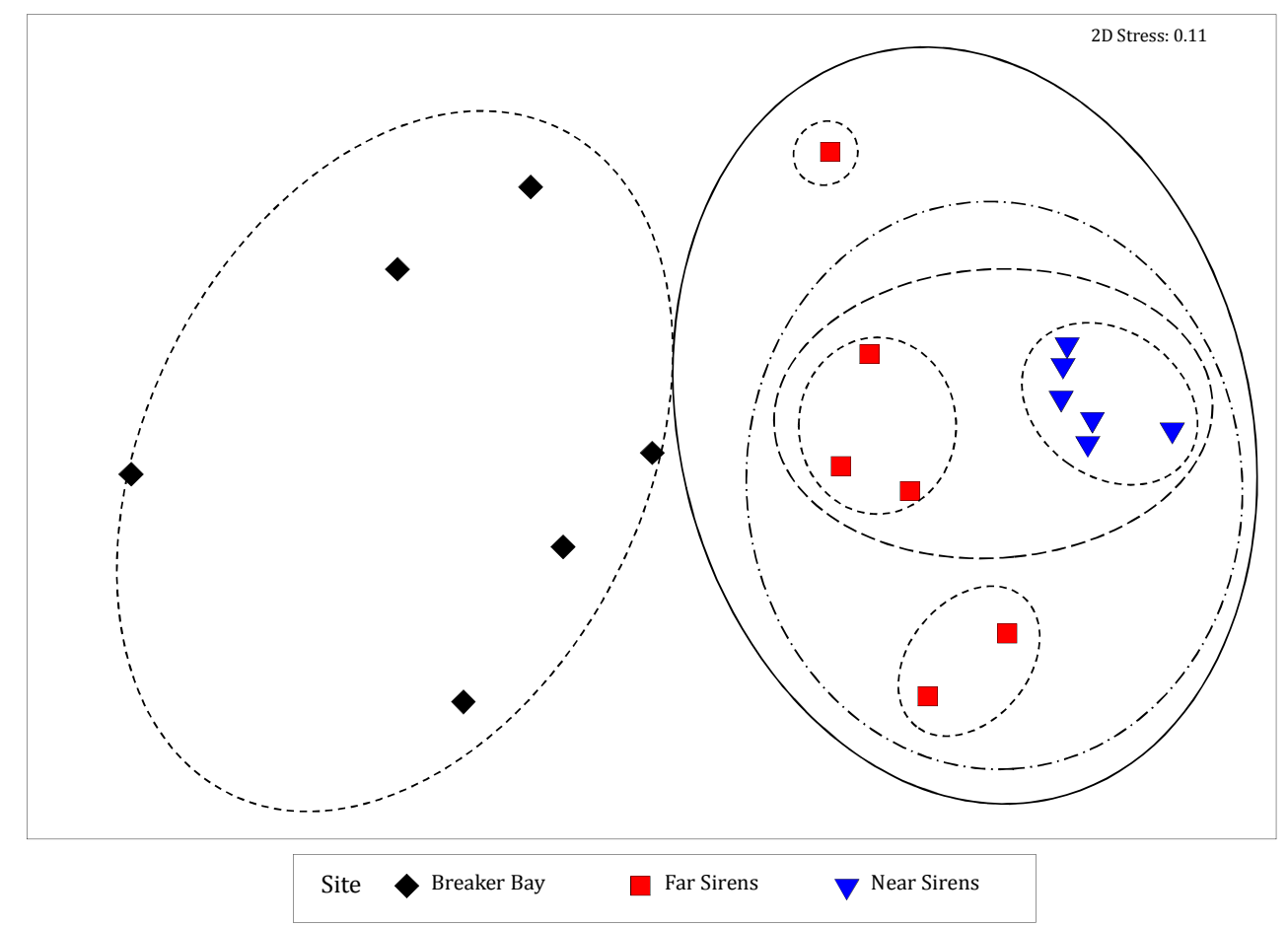

Fig. 3.8 Non-metric multidimensional scaling ordinations (nMDS plots in PRIMER) of the abundance within Wellington south coast sponge assemblages. These plots use zero adjusted BrayCurtis matrices which are $\log (\chi+1)$ transformed from the Far Sirens, Near Sirens and Breaker Bay based on abundance data with SIMPROF significant clusters ( $P<0.05 ; 1000$ Random permutations) shown by dotted ovals. 
The percentage cover data, which were $\log (\mathrm{X}+1)$ transformed, were significantly grouped by SIMPROF, with the Breaker Bay replicates, together with one replicate form the Far Sirens, forming one cluster, and the Near Sirens replicates forming another cluster. The Far Sirens data was split into two further significant subgroups $(\mathrm{P}<0.05)$ (Fig. 3.9). There were large differences between the replicates at the Far Sirens quadrats (they were spread over three significant clusters), while the quadrats for the Near Sirens site were more similar to each other.

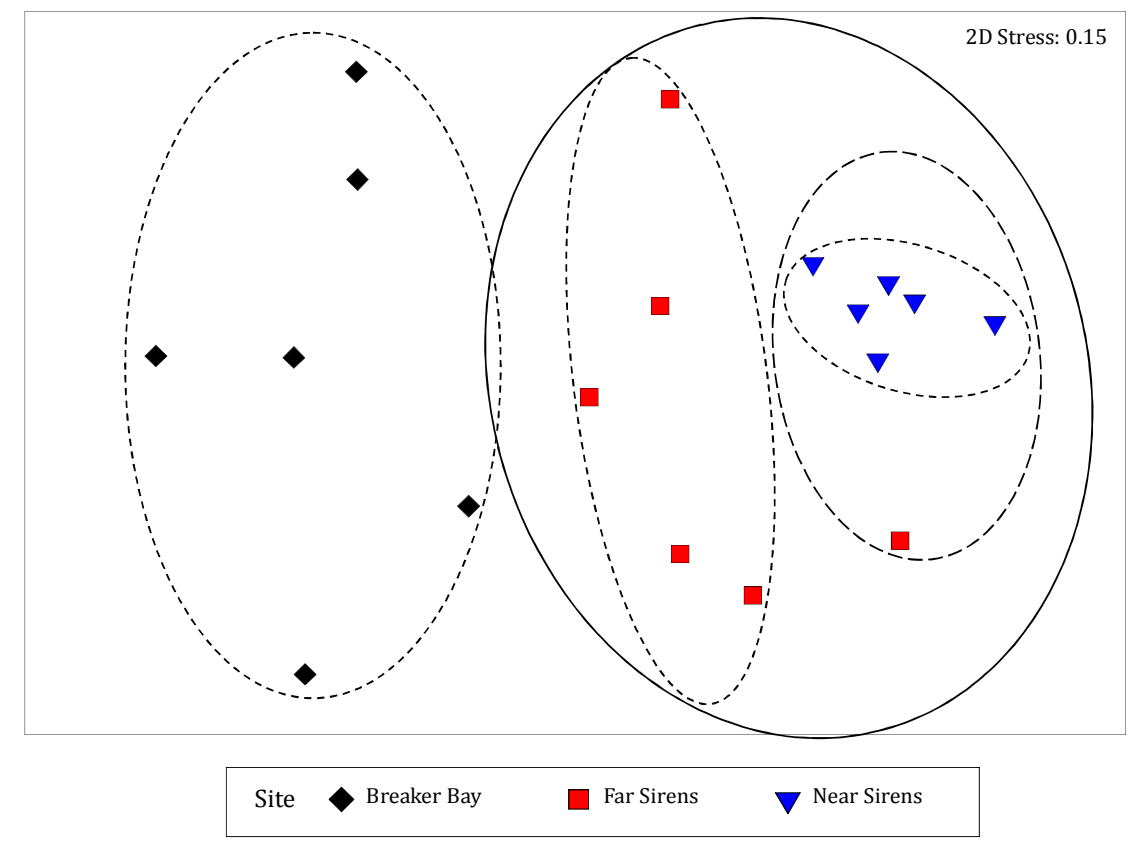

Fig. 3.9 Non-metric multidimensional scaling ordinations (nMDS plots in PRIMER) of the percentage cover within the Wellington south coast sponge assemblages. These plots use zero adjusted Bray-Curtis matrices that are $\log (\chi+1)$ transformed from the Far Sirens, Near Sirens and Breaker Bay based on percentage cover data with SIMPROF significant clusters $(P<0.05 ; 1000$ Random permutations) shown by dotted ovals.

ANOSIM showed that there were significant differences between all three sites based on the abundance dispersion-weighted data (Global test $\mathrm{R}=0.765$ $\mathrm{P}=0.001$ with 9999 permutations). Breaker Bay and the Near Sirens sites were very different $(\mathrm{R}=0.985 \mathrm{P}=0.002)$. Far Sirens and Near Sirens $(\mathrm{R}=0.626 \mathrm{P}$ $=0.002$ ) were significantly different, but had higher similarity than the other 
pairwise site comparisons; the Breaker Bay and Far Sirens were also significantly different $(\mathrm{R}=0.733 \mathrm{P}=0.002)$.

The $\log (\mathrm{X}+1)$ transformed percentage cover data used for the ANOSIM test showed that there were significant differences between all three sites (Global Test $\mathrm{R}=0.589 \mathrm{P}=0.001$ with 9999 permutations). Breaker Bay and the Near Sirens were very different $(\mathrm{R}=0.937, \mathrm{P}=0.002)$. The differences between the assemblage percentage cover at the Far Sirens site was significantly different from the Near Sirens and the Breaker Bay site $(R=0.457$ and $R=0.478$ respectively both at $\mathrm{P}=0.002)$. There was less difference identified from the percentage cover data than from the sponge density data, however, the differences in all cases were significant.

SIMPER analysis showed a similar pattern for both the percentage cover and density data (Tables 3.2-4). The Near Sirens sponge density replicates were most similar to other replicates at this site (62-75\% similarity) and least similar to the Breaker Bay replicates (18-36\%) (Table 3.2 \& 3.3). SIMPER analysis, using the percentage cover data, showed less variability within and between sites, however, the Breaker Bay site was less similar (40.99 Near Sirens and 49.36 Far Sirens) to the Sirens sites than the variation between the Breaker Bay replicates (Table 3.4). Stelletta sp. and Ancorina alata were the main species driving the differences in percentage cover of sponges within and between sites. 
Table 3.2 SIMPER analysis of the percentage similarity within and between sites in relation to sponge abundance (log transformed).

\begin{tabular}{|l|r|r|r|}
\hline \% Similarity & \multicolumn{1}{|l|}{ Far Sirens } & Near Sirens & Breaker Bay \\
\hline Far Sirens & 59.51 & \multicolumn{2}{|c|}{} \\
\hline Near Sirens & 56.04 & 75.1 & \\
\hline Breaker Bay & 47.45 & 36.42 & \\
\hline
\end{tabular}

Table 3.3 SIMPER analysis of the percentage similarity within and between sites in relation to sponge abundance (Dispersion Index down-weighted).

\begin{tabular}{|l|r|r|r|}
\hline \% Similarity & Far Sirens & Near Sirens & Breaker Bay \\
\hline Far Sirens & 41.33 & \multicolumn{2}{|c|}{} \\
\hline Near Sirens & 32.08 & 62.83 & \\
\hline Breaker Bay & 26.55 & 17.59 & 38.65 \\
\hline
\end{tabular}

Table 3.4 SIMPER analysis of the percentage similarity within and between sites in relation to sponge percentage cover.

\begin{tabular}{|l|r|r|r|}
\hline \% Similarity & Far Sirens & Near Sirens & Breaker Bay \\
\hline Far Sirens & 56.55 & \multicolumn{2}{|c|}{} \\
\hline Near Sirens & 53.96 & 69.73 & \\
\hline Breaker Bay & 49.36 & 40.99 & 54.25 \\
\hline
\end{tabular}

As shown previously (Fig. 3.7), when the data is not down-weighed with a dispersion index, the calcareous sponges dominate the abundances, particularly at the Near Sirens sites. When the abundance values are adjusted using downweighting, the calcareous sponges (especially the Clathrina spp.) are the main contributors to the differences between the Near Sirens and the other two sites. Halisarca sp. and Mycale sp. were the main contributors to the differences between Breaker Bay and the Far Sirens sites, with Halisarca sp. being the main species present across all the replicates at the Far Sirens and Mycale sp. being the characterising species for the Far Sirens replicates. 
RELATE was used to compare the rank correlations between the similarity matrices (which the MDS plots are a visual representation of) for abundance and percentage cover. There was a highly significant correlation $(\mathrm{R}=0.816$ and $\mathrm{P}<0.001$ ) between the percentage cover and density data therefore the patterns they show are very similar. 


\subsection{DISCUSSION}

This work provides the first quantitative survey of sponge assemblages on the Wellington south coast, New Zealand. Sixty-five different sponge species from 27 families and eleven orders of demosponges and four families and three orders of calcareous sponges were reported, which is relatively high in comparison with other similar-sized sampling areas from temperate regions, including the few areas sampled quantitatively in New Zealand (Table 3.5). Forty-three of the species were found within the quantitative survey with a mean of $18.89 \pm 1.25$ species per quadrat across all three sites, with no significant differences in the number of species present between sites. Percentage cover and sponge density were significantly different between all sites, as was assemblage structure between some site pairs.

There are over 800 known sponge species from the New Zealand biogeographic region (Chapter 2). This is high in relation to other well-known temperate areas of similar size, such as the UK and Ireland where approximately 400 species have been described (Picton \& Goodwin, 2007; Kelly et al., 2009), and these areas have been much more extensively studied. Within the 800 known species in New Zealand waters over 95\% are considered to be endemic at the national level (Kelly et al., 2009). There have been few intensive regional surveys and most reports of sponge diversity are in grey literature. Examples of these include: 57 species recorded from the Pariokariwa reef, North Taranaki (Battershill \& Page, 1996); 215 species recorded between North Cape and Cape Reinga at the very tip of the North Island (Cryer et al., 2000); and 84-170 species recorded from the Cape Rodney to Okari Point Marine Reserve (about $90 \mathrm{~km}$ north of Auckland) (Ayling, 1979; Pritchard et al., 1984). It is hard to compare these other New Zealand values with the values from the WSC directly, as they sampled different habitat types with variable levels of sampling effort, however, these results combined with this study suggests that there are multiple areas of high sponge richness around the North Island of New Zealand. Currently, there are no known regional data sets for sponge richness from the South Island of New Zealand for comparison. 
Table 3.5. Global values for sponge species richness. All values shown are taken from a depth of 12$18 \mathrm{~m}$ over similar sized sampling areas on bedrock substrata (updated from (Bell \& Barnes, 2000a)). Although other sponge richness values are available they are not directly comparable due to varying sampling effort and habitat type.

\begin{tabular}{|c|c|c|}
\hline Location & Species Richness & Source \\
\hline Hoga Island (Indonesia) & 100 & (Bell \& Smith, 2004) \\
\hline New South Wales (Australia) & 82 & (Roberts et al., 2006) \\
\hline Havana (Cuba) & 80 & (Alcolado, 1990) \\
\hline Lough Hyne (Ireland) & 77 & (Bell \& Barnes, 2000a) \\
\hline Quirimba (Mozambique) & 73 & (Bell \& Barnes, 2000a) \\
\hline Wellington (New Zealand) & 65 & This study \\
\hline Skomer Island (Wales, UK) & 57 & (Bell et al., 2006) \\
\hline Southern Florida (USA) & 43 & (Schmahl, 1990) \\
\hline Kenya & 25 & (Barnes \& Bell, 2002) \\
\hline Sharm el Sheik (Eygpt) & 21 & Bell (unpublished data) \\
\hline Signy (Antarctica) & 19 & (Bell \& Barnes, 2000a) \\
\hline San Blas (Panama) & 17 & (Bell \& Barnes 2000a) \\
\hline Venezuela & 15 & (Diaz et al., 1990) \\
\hline Cork (Ireland) & 13 & (Bell \& Barnes, 2000a) \\
\hline Sussex (England, UK) & 12 & (Bell \& Barnes, 2000a) \\
\hline Sal (Cape Verde) & 9 & (Bell \& Barnes, 2000a) \\
\hline Cornwall (England, UK) & 6 & (Bell \& Barnes, 2000a) \\
\hline
\end{tabular}

The sponge species present on the WSC are likely to be a mixture of North and South Island species as the WSC is an area where three currents meet (Heath, 1971). The dynamic high-energy current environment may result in a sponge assemblage composed of species at the northern and southern limits of their distributions, such as has been reported for macroalgae species (Nelson, 2008). The sponge species pool on the Wellington south coast may be linked to the biographic transition zone for cold (South Island) and warm (North Island) 
species, although further comparisons between regional species composition data is required to confirm this.

The sites used for quantitative analysis were chosen as they appeared to be similar to each other (similar depths and substrata), such that they could be considered replicates for the Wellington region for a larger temporal investigation. Despite appearing visually similar on first examination, the MDS plots and the ANOSIM for both density and percentage cover data indicated clear differences between all three sites. This difference is also supported by the univariate data as the Near Sirens had significantly higher density of sponges and percentage cover than the other two sites. The other two sites also had less overlap in assemblage composition, which was shown by the SIMPROF cluster analysis placing one of the replicates from Breaker Bay within the Far Sirens Group and one of the Far Sirens quadrats into a separate group of its own. This can be explained by the high levels of within site variability, particularly at Breaker Bay, shown in the MDS plot, compared to the other sites. However, the SIMPROF grouped all the Breaker Bay replicates together, and they were significantly different from the Sirens sites. The down-weighted abundance data reduces the 'error' variance attributable to highly variable species and reduces the noise caused by species that have clumped distributions. There was a significant difference in the percentage cover of sponges between Breaker Bay and the two Sirens sites, while the two Siren's sites had similar percentage cover.

The RELATE test showed that the data for percentage cover and density were highly correlated across the three sites. The similarities between replicates (irrespective of measurement type) are likely to be driven by a common set of environmental drivers, therefore explaining the similar site patterns obtained for species with higher densities as for species with higher percentage cover as they both appear to respond in a similar way.

All sites had higher between site variability than within site variability according to the SIMPER analysis. Breaker Bay had the highest within site variability, while the Near Sirens site had the lowest within site variability for 
both percentage cover and sponge density data. Both of the Siren's sites were also more similar to each other than to the Breaker Bay site. The Sirens sites are approximately $200 \mathrm{~m}$ apart, while the Breaker Bay site is about $6 \mathrm{~km}$ away from the other two sites, so this result is perhaps not surprising. The environmental drivers are likely to be different in terms of the nutrient content of the water between the Sirens sites and Breaker Bay, particularly as there is low organic water content in the waters along the WSC extending from the Wellington Harbour, which is closest to Breaker Bay. The WSC waters have low levels of organic matter including total particulate and dissolved organic matter, when compared with other temperate regions, however, seston values are particularly low compared to other similar temperate areas. These low levels of seston are thought to limit the number of other suspension feeders such as mussels, which are very rare on the WSC (Gardner, 2000; Gardner \& Thompson, 2001; Helson et al., 2007). Sponges can phagocytise the smallest fractions of the nano- and picoplankton (particles $<2 \mu \mathrm{m}$ ) including bacteria and possibly viruses (Booth, 1975; Pile et al., 1996; Ribes et al., 1999; Perea-Blázquez et al., 2010). Therefore even though the waters on the WSC may have low levels of organic material, particularly its seston content, sponges can exploit the low levels of nutritional content in the form of viruses and bacteria and proliferate where other suspension feeders cannot survive.

Other studies of sponge assemblage spatial variation in temperate waters have also shown considerable spatial variation between locations (Kennelly \& Underwood, 1992; Roberts \& Davis, 1996). The factors likely to be responsible for this variation include larval supply, recruitment, physical disturbance and biological interactions (Underwood et al., 1991). The south coast is a very active hydrodynamic area and variation in the energy reaching the sites and scouring away near shore sponge assemblages is likely to be high. The reasons for spatial variation in sponge species could be due the naturally patchy distribution of sponge species and their short range dispersal patterns that result in the formation of local clusters of specific species. For example, the budding of Stelletta sp. and Ancorina alata would explain their local high percentage cover at some of the sites (Fell, 1993). 
The most abundant sponges by density and percentage cover were encrusting species of the genus Clathrina. Clathrina spp. are calcareous sponges that are found all over the world, however, they are difficult to identify (Klautau \& Valentine, 2003). Other calcareous sponges were also among the top fifteen most abundant species including Leucosolenia spp. and Leucetta sp. These species are also found in other sites with high sponge richness on the North Island of New Zealand including the Pariokariwa reef off North Taranaki, White Island and Volkner Rocks in the Eastern Bay of Plenty and the Poor Knights off the East coast of Northland, but they are much rarer than those found on the WSC, where they dominate the sponge assemblages.

Calcareous sponges, which dominate by abundance on the WSC, have been reported to increase in abundance and percentage cover in winter, with a die-off in the summer in other temperate locations, which is the opposite pattern to many of the Demosponges (Dendy, 1914; Orton, 1914; Burton, 1949; Johnson, 1979). The present survey was carried out in April, which is the autumn in New Zealand, and therefore the waters are just starting to cool. It will be interesting to see if calcareous sponges dominate the assemblages throughout the year or if they have seasonal changes in abundance as has been reported from other locations. Calcareous sponges are thought to be short-lived (less than one year in some cases) in comparison with Demosponges that can live much longer, some over a hundred years (Reiswig, 1973; Tuzet, 1973; Johnson, 1979). Clathrina clathrus has been shown to have a hypo-active phase over winter allowing it to control its metabolism and maintain biochemical homeostasis over the winter months (Burlando et al., 1992).

The Wellington south coast supports a diverse sponge assemblage with up to 500 sponges per $\mathrm{m}^{2}$, covering over $50 \%$ of the substratum at some sites. Although the species density is similar across the three sites the species composition, their abundances and percentage coverage vary significantly. All the sites are atypically dominated by calcareous species, in particular of the genus Clathrina, which appears to be unusual for the North Island or New 
Zealand generally. Reasons for the high abundance and density of Clathrina spp. could be due to their ability to be hypo-active during the winter months and therefore be spatially dominant at a time when other Demosponges and other filter feeders might be regressing due to less food in the water and colder temperatures. Chapter four will investigate the temporal diversity of sponge assemblages on the Wellington south coast. 
Chapter 3 Spatial variability of sponge assemblages on the Wellington South Coast 
CHAPTER 4

TEMPORAL VARIABILITY IN SPONGE ASSEMBLAGES ON THE WELLINGTON SOUTH COAST, NEW ZEALAND 


\subsection{ABSTRACT}

This chapter provides the first quantitative survey of the temporal variation of the sponge assemblages on the Wellington South Coast, New Zealand. Permanent photoquadrats were set up at three sites at a similar depth and aspect along the Wellington South Coast. The multi and univariate data showed that there were significant site differences in the sponge assemblages and the interaction between site and season differences was also significant. The interaction of the site and season means that each site changed differently in relation to the season and it is not possible to isolate the effect of either site or season individually; therefore each of the sites responded in a different way to seasonal changes. The species richness was significantly different between seasons. SIMPER analysis showed that some of the calcareous species were the most important in terms of explaining both the similarities within sites and the differences between sites, as well as seasonal similarities and differences. Total monthly rainfall, highest speed wind gust, mean monthly air temp, seawater temperature and mean air pressure were all significantly correlated with the patterns of the sponge assemblage diversity. The best single environmental correlation was with the seawater temperature data. The sponge data collected during this study did not show any consistent seasonal variation patterns across the three sites studied. 


\subsection{INTRODUCTION}

The spatial distribution of the sponge fauna of the Wellington South Coast, New Zealand was discussed in the third chapter of this thesis. That chapter described the spatial diversity patterns within the Wellington region, in part because the Cook Strait bioregion has high values of AvTD and VarTD for sponge biodiversity. This chapter investigates the temporal variability of the sponge assemblages of the Wellington South Coast.

The majority of studies examining variability in sponge assemblages have examined a single geographic location at a single sampling interval (Barnes \& Bell, 2002; Cleary et al., 2005; Janussen \& Tendal, 2007; Picton \& Goodwin, 2007; Duckworth et al., 2008; Berman \& Bell, 2010) or a specific genus (Duckworth \& Battershill, 2001; Klautau \& Valentine, 2003), family (Plotkin, 2004) or functional role (Lemloh et al., 2009) at the regional spatial scale. Temporal variation has mostly been explored at the species level (Garrabou \& Zabala, 2001; de Caralt et al., 2008; Koopmans \& Wijffels, 2008; McMurray et al., 2008) and to date, there have been relatively few studies examining temporal variation in entire sponge assemblages over more than a year (but see chapters 5 and 6 in this thesis; Sara, 1970; Ayling, 1983b, a; Pansini \& Pronzato, 1985; Bell et al., 2006; Carballo et al., 2008). It is important to understand the temporal variation of assemblages as the loss of sponge biodiversity and abundance is difficult to quantify without prior monitoring as sponges disappear quickly when they die leaving no trace behind (Wulff, 2006a). Research from the Mediterranean has shown slow growth within sponge assemblages with no clear seasonality and only some sponge tissue regression, while the abundance of different sponge species remained similar throughout an eight year study (Sara, 1970; Pansini \& Pronzato, 1985). Similar results showing little overall change have also been reported from sites in the Atlantic (Fowler \& Laffoley, 1993; Hiscock, 1994) and sites in the Caribbean (Hughes, 1996). However, even though the overall abundance of sponge individuals and 
percentage cover within sponge assemblages remained relatively constant in the Caribbean sites, the actual individual sponges were very variable suggesting recruitment and mortality were in equilibrium in these assemblages (Hughes, 1996). However, more recent work in the Atlantic, Caribbean and Pacific suggests that more rapid changes in sponge assemblages are common and recruitment and mortality do not always occur in equilibrium and therefore the overall assemblage composition can show dramatic changes (Bell et al., 2006; Wulff, 2006a; Carballo et al., 2008). Assemblage changes have been correlated to processes occurring over short temporal scales, such as wind speed and sediment deposition or through large-scale structuring factors, such as the Southern Oscillation (Carballo et al., 2008). However, Carballo et al., (2008) only found a correlation between sponge assemblage changes and the Southern Oscillation.

Variation in sponge diversity, distribution and abundance is likely to influence other benthic organisms due to the important functional roles that sponges fulfill (Pang, 1973; Rützler, 1975; Wulff \& Buss, 1979; Corredor et al., 1988; Ribeiro et al., 2003; Bell, 2007a; Bell \& Carballo, 2008; Bell, 2008). Sponge assemblages are influenced by a number of physical factors including: depth (Wilkinson \& Evans, 1989; Duckworth et al., 2004; Cleary et al., 2005); water flow (Leichter \& Witman, 1997; Duckworth et al., 2004); temperature (Riisgard et al., 1993; Barnes, 1999); light intensity (Alcolado, 1994); sedimentation (Sara \& Vacelet, 1973; Wilkinson \& Vacelet, 1979; Zea, 1994; Ilan \& Abelson, 1995) and salinity (Fell et al., 1989; Leamon \& Fell, 1990; Barnes, 1999). Biological factors influencing sponge assemblages include: predation (Wulff, 1994, 1995, 1997a, 2000); mutualistic and symbiotic associations (Rosell \& Uriz, 1992; Arillo et al., 1993; Wulff, 1997b); concentration and plankton diversity (Pile, 1996; Ribes et al., 1999; Ribes et al., 2003); spatial competition (Jackson, 1977; Aerts, 1998; Fagerstrom et al., 2000); and disease (Smith, 1941; Galtsoff, 1942; Pronzato, 1999; Wulff, 2007). All of the above factors vary temporally on some scale for subtidal assemblages. For example, even the depth of subtidal assemblages changes temporally on the short-term scale with tides and on the 
long-term scale with tectonic shifts of continental plates. However, the factors which are likely to be the most important for seasonal and annual temporal change are the physical factors temperature, sedimentation and light intensity and the biological factors disease, predation and food availability. There has been some discussion regarding the relative roles of biological and physical factors in controlling sponge assemblages in different geographic regions (Bell \& Carballo, 2008). Biological factors may be more important than physical factors in determining tropical sponge assemblage structure in the Caribbean, however, physical factors have been found to be most important in the NE Atlantic and Eastern Pacific (Wulff, 1997a; Bell \& Barnes, 2000a; Wulff, 2000; Bell \& Barnes, 2003a; Carballo, 2006). It is important to note that all the above studies focused on spatial not temporal variation and different factors may be important in determining assemblages at different times of the year or from year to year.

Some of the sponge species and typical sponge habitats on the Wellington South Coast (WSC) have recently been described, although work focused on relatively few common species from only a few sites (Duckworth \& Battershill, 2001; Duckworth et al., 2004; Berman et al., 2008; Perea-Blázquez et al., 2010). In chapter 3 the most abundant sponges by density and percentage cover were encrusting species of the genus Clathrina and other calcareous species were also abundant. The survey for chapter three took place in April 2008, which is autumn in New Zealand. Calcareous sponges have been reported to increase in abundance and percentage cover in winter, with a die-off in the summer in other temperate locations, which is the opposite pattern to many of the demosponges (Dendy, 1914; Orton, 1914; Burton, 1949; Johnson, 1979). Both calcareous and demosponges have been found to feed on picoplankton in temperate waters (Ribes et al., 1999; Hanson et al., 2009; Perea-Blázquez et al., 2010). However, calcareous sponges tend to have lower removal efficiencies then demosponges according to a study on two calcareous species from the Wellington South Coast (Perea-Blázquez et al., 2010). Sponges are an important link in taking advantage of the picoplankton and facilitating benthopelagic 
coupling between the trophic levels (Bell, 2008). Clathrina clathrus, which is a calcareous sponge, has been shown to have a hypo-active phase over winter allowing it to control its metabolism and maintain biochemical homeostasis over the winter months (Burlando et al., 1992). Some demosponges, such as Halisarca caerulea, can assimilate dissolved organic matter and provide a net source of particulate food into the marine environment (De Goeij et al., 2009). In Wellington Haliclona venustina exhibited ammonia excretion throughout the seasons although other demosponges and calcareous sponge did not have a net uptake or output of a range of nutrients (Perea-Blázquez, 2011). Calcareous sponges are thought to be short-lived (less than one year in some cases) and generally reach a smaller adult size of less than ten $\mathrm{cm}$ in comparison with demosponges that can live much longer, some up to hundreds of years (Reiswig, 1973; Tuzet, 1973; Johnson, 1979). Therefore a calcareous sponge dominated system is likely to have a high turnover of sponges while demosponges may have a longer turnover period and be able to grow to larger sizes. Therefore, it is important to understand the seasonal variability in abundance of calcareous and demosponges so they can be included into monitoring programmes appropriately and acknowledge the differing functional roles they play in benthic ecosystems.

This chapter explores the temporal variation along the Wellington South Coast The aims of this study were:

1. To measure the patterns of seasonal variability in sponge assemblages from the Wellington South Coast.

2. To elucidate if the patterns of variability are different for demosponge and calcareous species within the assemblages.

3. To identify the environmental factors that best explain patterns of the variation in Wellington sponge assemblage composition, diversity and abundance. 


\subsection{METHODS}

\subsubsection{STUDY AREA}

Sponge assemblages were quantitatively sampled at three sites along the Wellington South coast that were chosen after the preliminary biodiversity survey described in chapter three. These were: Near Sirens (4120'56.43"S,

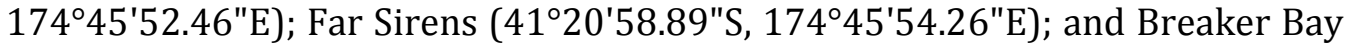
(4119'58.28"S, 17449'52.98"E) (see Fig 3.1). These sites were chosen as they were visually similar sponge-dominated sites with continuous similar vertical bedrock habitat forming channels at a similar depth $(8-10 \mathrm{~m})$ along the Wellington South Coast (WSC). All three sites were found to be significantly different from each other in the spatial survey in chapter 3. Near Sirens is about $50 \mathrm{~m}$ offshore and is perpendicular to the coast. Far Sirens is about $200 \mathrm{~m}$ further offshore than the Near Sirens and is in a wider channel parallel to the coast. Breaker Bay is about $6 \mathrm{~km}$ away from the other two sites and along a rocky channel which breaks the surface about $150 \mathrm{~m}$ offshore. All three sites are on Wellington greywacke sedimentary rock which is mostly sandstone and sandstone-argillite dominated. The sessile communities at all three sites include high densities of sponges, ascidians, bryozoans and algae and since October 2008 Near Sirens and Far Sirens have been within the newly gazetted Taputeranga marine reserve.

The study area has a south to south-east aspect, and is exposed to frequent high-energy south-easterly swells and storm episodes interspersed by northwesterly winds. There are three separate water bodies that combine to form the Cook Strait current that flows past the study sites: the Southland Current (a mix of subtropical and sub-Antarctic water) that flows northward up the east coast of the South Island and westward into the Cook Strait, the East Cape Current (subtropical water) that flows south along the east coast of the North Island and then westwards into Cook Strait and the D’Urville Current (warm subtropical 
water) that flows from north to south from the Tasman sea (Heath, 1971). The winds and tides are also strengthened through the narrowing of the Cook Strait (Carter \& Lewis, 1995). In addition, local wind waves compound the persistent southerly swell. These winds are funnelled either north or south by the local topography. As a result, the coastline is constantly exposed to high energy swell coming from the south (Heath, 1985). Weather in Wellington is very changeable and there are rarely long settled periods. Tidal currents in the region can reach speeds of up to $360 \mathrm{cms}^{-1}$ (Carter, 2008). Maximum wave heights can exceed $13 \mathrm{~m}$ during large storm events, while wave height (the highest one-third of the waves) can exceed $8 \mathrm{~m}$ (Carter et al., 2002). This type of wave regime inevitably influences intertidal and subtidal community structure (Pande \& Gardner, 2009).

\subsubsection{SURVEY METHODOLOGY}

Six $0.5 \mathrm{~m}^{2}$ haphazardly placed quadrats (within the available vertical bedrock) were marked in May 2008 (six quadrats were required to record the species richness and density of sponges in the spatial survey see fig 3.4 and 3.5).These quadrates were surveyed approximately every three months and each sampling survey was within a maximum of a four week period apart. Each quadrat was divided into $25 \times 10 \mathrm{~cm}^{2}$ sections and each section was photographed. Within each section, the numbers of sponge patches were recorded from the pictures. If a sponge overlapped two sections the patch was only counted once. The sponge density, mean species richness and Shannon index were calculated for each site for each of the different sampling times. In chapter 3 it was found that there was a significant correlation between the similarity matrices for sponge density and percentage cover data across the three sites sampled $(\mathrm{R}=0.816$ and $\mathrm{P}<0.001)$ therefore the sponge density data will be used within this chapter.

PRIMER (ver. 6) with the PERMANOVA add-on was used to carry out a nonparametric equivalent of a univariate ANOVA (permutational MANOVA) using 
the species richness and sponge density data as response variables. This method calculates P-values using permutations, thus needing no assumption of a normal distribution for the data. The model used for the PERMANOVA design is shown in Table 4.1. PERMDISP was used to test for the homogeneity of beta diversity within and between the sites and within and between seasons. This was in order to understand if all three sites were actually part of one location $(\alpha$ diversity) or if the three sites were different from each other and therefore representative of regional diversity ( $\beta$ diversity). Definitions of the spatial scale terminology follow Gray (2001), which were modified from his earlier classification (Gray, 1997).

Table 4.1. Design for the PERMANOVA model for the analysis of the multivariate species and morphological data in response to the factors listed.

\begin{tabular}{|l|c|c|c|}
\hline Factors & Abbreviation & Type & Levels \\
\hline Site & Si & Random & 3 \\
\hline Quadrat & Qu & Fixed & 18 \\
\hline Year & Ye & Fixed & 2 \\
\hline Season & Se & Random & 4 \\
\hline
\end{tabular}

PRIMER was used to carry out ordination by non-metric Multi-Dimensional Scaling (MDS) on a dissimilarity matrix created from a Bray-Curtis similarity analysis to compare species assemblages between all three sites, between years and seasons to look for temporal and spatial patterns. The species data were $\log (\mathrm{x}+1)$ transformed to reduce the influence of rare species. MDS plots were created for the complete data set, and also for sub groups; site and season.

SIMPER analysis was used to elucidate the relative contribution of each species to the average Bay-Curtis similarity between the three sites and also the dissimilarity between the seasons. This method determines which species were most responsible for any differences between the groups. 
PERMANOVA was used to distinguish which factors or interacting factors were significant in determining the spatial and temporal differences between the sponge assemblages. Interactions between factors occur when there is an additive or multiplicative effect when there are changes in two or more factors. The change in sponge assemblage variability at a site depends on the change in seasonal variability in general on the Wellington South Coast and an additive or multiplicative effect of local seasonal variability at each site.

The model used for the PERMANOVA design is the same as for the univariate assemblage structure in Table 4.1 The test statistic for PERMANOVA is the pseudo F-ratio, where a large pseudo F-ratio indicates that a factor such as location or season groups the quadrats closer in multivariate space in comparison to a random distribution and therefore differs in assemblage composition. The significance of the pseudo F-ratio is tested using a permutation test that randomly shuffles the sample labels within and among groups and calculates the pseudo F-ratio for 9999 arbitrary reassignments of the Bray Curtis resemblance matrices for species diversity/morphological assemblages. The pseudo F-ratios of these randomly assigned communities were then compared to the pseudo F-ratio of the observed community to calculate the significance level of the test (Anderson, 2001).

The density of calcareous and demosponges sponges were also compared between sites and seasons to see if they varied in a similar magnitude of change and a permutational MANOVA using the model in Fig 4.1 was used to see if the calcareous and demosponge data was related to site, seasonal or their interaction.

\subsubsection{ENVIRONMENTAL DATA}

Water temperature data were recorded using in situ HOBOware data loggers at each of the three sites. Data were available from the end of June 2008-May 2009 for the Near Sirens and Far Sirens sites, however, at Breaker Bay the first Hobo went missing before collection and the second Hobo malfunctioned and lost all 
the remaining data. The mean seawater temperature for the month is calculated from the reading taken each day at 9am in the morning from each of the sites for a total of 30 days. Additional environmental data were collected from the Wellington airport meteorological station by NIWA, which are published online and are for public use in the National Climate database (NIWA, 2011). These include monthly air temp (mean, max and min), air pressure and maximum wind gust speed. These variables were used in part to see if they could be a proxy for sea temerature and also to see the influence of storms on the assemblages. All the variables were normalised and then as all three air temperature variables were highly correlated mean monthly air temp was used to represent all air temperature variables.

The DistLM3 (BEST) function in PRIMER was used to determine any correlations between individual or combination of environmental variables and the changes in the species and assemblages. This uses the Spearmans rank coefficient and the BIOENV procedure to find the highest correlation for a set of environmental variables (in this case up to 3 of the variables) and the significance of the correlation was estimated using 9999 permutations. 
Chapter 4 Temporal variability in sponge assemblages on the Wellington South Coast, New Zealand

\section{$4.4 \quad$ RESULTS}

\subsubsection{TEMPORAL VARIABILITY OF THE ASSEMBLAGES}

The mean species richness' across the three sites and during the different seasons found per $\mathrm{m}^{-2}$ are shown in Fig. 4.1. There was a significant difference between the number of species in the different seasons $(\mathrm{F}=3.56 \mathrm{P}=0.045)$ and also for the interaction of season and site $(\mathrm{F}=7.23 \mathrm{P}=0.001)$ using a permutational MANOVA. The interaction between site and season means that each of the sites responded differently in relation to season. There were no significant differences between the species richness and different sites, years or their interactions.

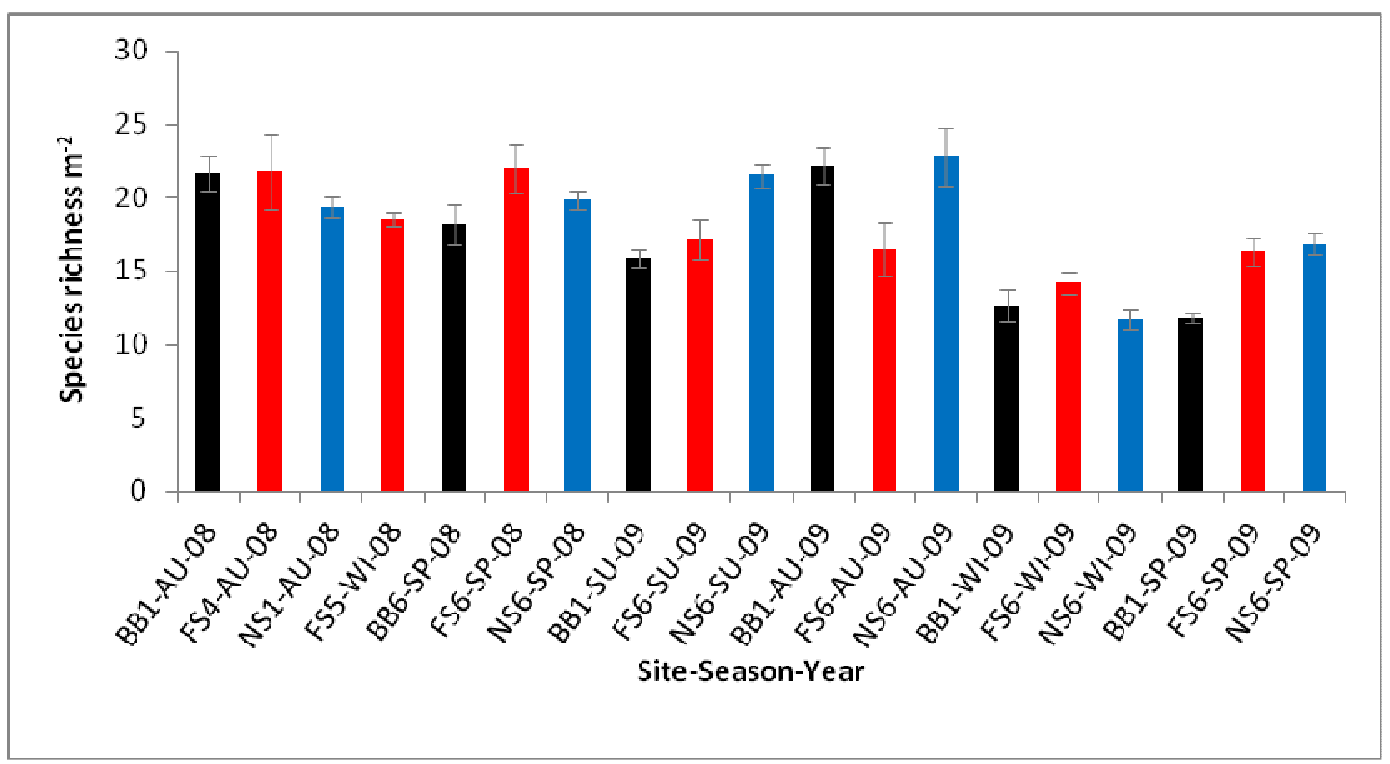

Fig. 4.1 Sponge species richness ( \pm S.E.) for the Wellington South Coast. The mean species richness $( \pm$ S.E.) for Breaker Bay (black), Far Sirens (red) and Near Sirens (blue) across the four seasons.

The mean sponge density across the three sites and during different seasons split into calcareous and demosponges found in $\mathrm{m}^{-2}$ are shown in Fig. 4.2. There was a significant difference between the total sponge density and the different 
Chapter 4 Temporal variability in sponge assemblages on the Wellington South Coast, New Zealand

sites ( $\mathrm{F}=6.32 \mathrm{P}=0.021999$ Permutations) and the interaction between season and site $(\mathrm{F}=15.667 \mathrm{P}=0.001999$ Permutations) using a permutational MANOVA. There were no significant differences between the total species richness and season, year or the interaction term.

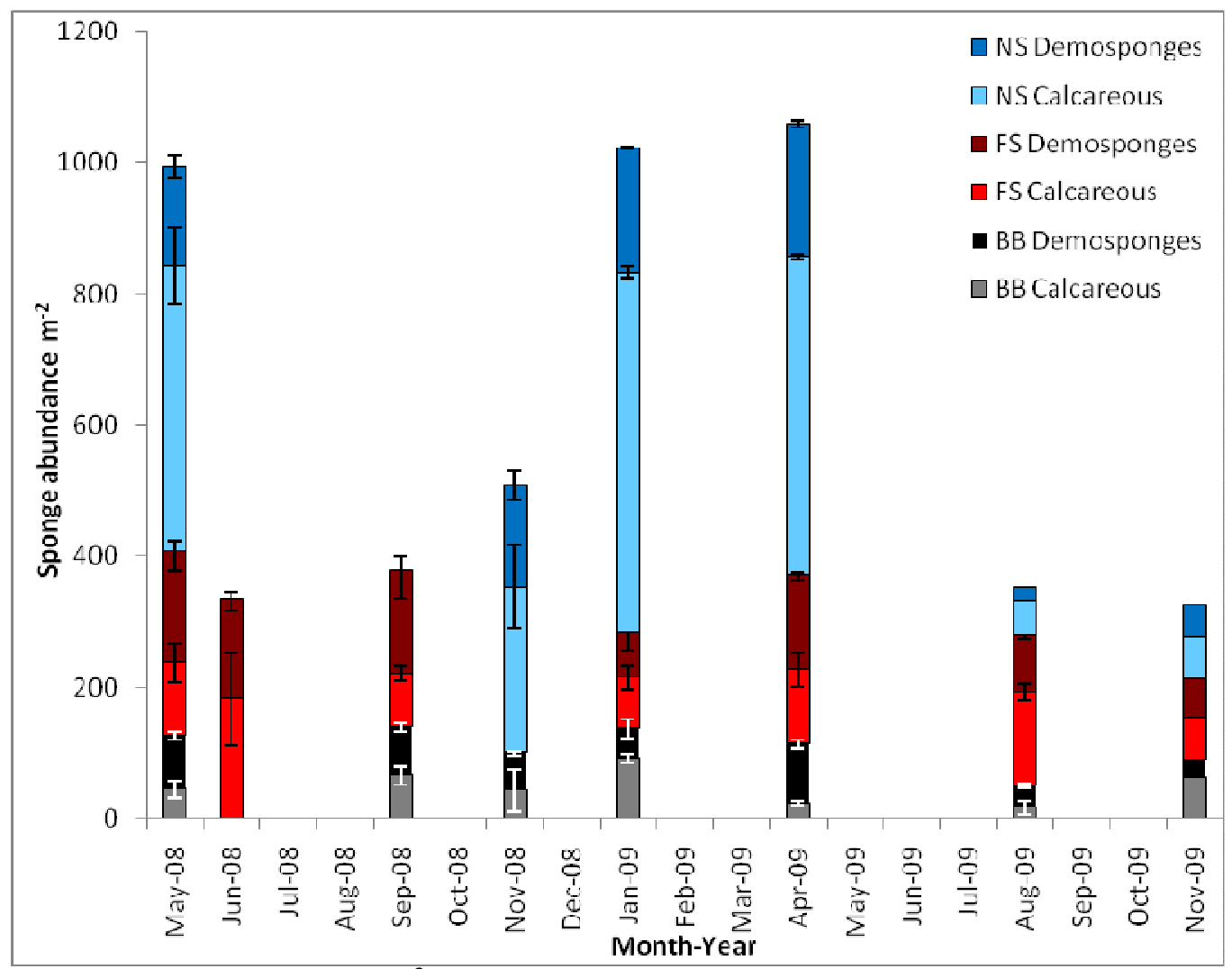

Fig. 4.2 Sponge abundance $\mathrm{m}^{-2}$ ( \pm S.E.) for the Wellington South Coast. The mean sponge abundancess (split into calcareous and demosponges) ( \pm S.E.) for Breaker Bay (BB), Far Sirens (FS) and Near Sirens (NS) from May 2008-November 2009.

There was a significant difference between the calcareous sponge densities between the different sites $(\mathrm{F}=6.92 \mathrm{P}=0.016999$ permutations $)$, quadrats ( $F=2.13 \mathrm{P}=0.01999$ permutations) and also due to the interaction term for season and site ( $\mathrm{F}=13.26 \mathrm{P}=0.001999$ permutations). Season, Year and their interaction term were not significant. Near Sirens had the highest abundances of calcareous sponges initially and Breaker Bay the lowest however there was a dramatic delcline in calcareous sponges at the Near Sirens site while those at the Far Sirens and Breaker Bay remained more consistent. 
There were no significant differences between site, season, quadrat or year factors (or the interaction terms) for demosponge density. However, there was a significant difference between demosponge density for the interaction term site and season ( $\mathrm{F}=9.042 \mathrm{P}=0.001999$ permutations).

As the changes in season or year were not significant in predicting changes in the sponge assemblages across sites it is hard to identify any overall seasonal patterns as the sponge abundances at the sites fluctuated in different ways. For the Near Sirens and Breaker Bay the total sponge abundances were high compared to other within site sampling periods in the summer (Near Sirens Jan $2009-740 \pm 54$ S.E. sponges $\mathrm{m}^{-2}$ and Breaker Bay January 2009 with $138 \pm 35$ S.E. $\mathrm{m}^{-2}$ ). However, the Far Sirens had one of its lowest total sponge abundances in Jan 2009 (146 \pm 29 S.E. sponges $\mathrm{m}^{-2}$ )

Total sponge abundances were also high in autumn for the Far Sirens and the Near Sirens sites (Far Sirens May 2008 - $281 \pm 60$ S.E. sponges $\mathrm{m}^{-2}$ and April $2009-257 \pm 45$ S.E. sponges $\mathrm{m}^{-2}$ and Near Sirens May $2008-585 \pm 71$ S.E. sponges $\mathrm{m}^{-2}$ and April $2009-689 \pm 71$ S.E. sponges $\mathrm{m}^{-2}$ ).

The Far Sirens had the highest mean density of sponges in the winter sampling in June 2008 (334 \pm 87 S.E. sponges $\mathrm{m}^{-2}$ ), and higher than average in August 2009 with $229 \pm 32$ S.E. sponges $\mathrm{m}^{-2}$. In contrast, Breaker Bay and Near Sirens had their lowest sponge densities in August 2009 (Breaker Bay $49 \pm 10$ S.E. sponges $\mathrm{m}^{-2}$ Near Sirens $76 \pm 9$ S.E. sponges $\mathrm{m}^{-2}$ ).

Calcareous sponges were consistently at higher densities than demosponges at the Near Sirens sites across seasons and showed a similar pattern to the total sponge abundance for the Near Sirens with the highest densities in summer (549 \pm 64 S.E. sponges $\mathrm{m}^{-2}$ January 2009) and autumn (487 \pm 63 S.E. sponges $\mathrm{m}^{-2}$ April 2009). Breaker Bay also had the highest density of calcareous sponges in summer (January 2009 with $92 \pm 31$ S.E. sponges $\mathrm{m}^{-2}$ ). The Far Sirens had the highest abundances of calcareous sponges in winter ( $182 \pm 70$ S.E. sponges $\mathrm{m}^{-2}$ June 2008 and $142 \pm 26$ S.E. sponges $\mathrm{m}^{-2}$ in August 2009) and the second lowest 
density of calcareous sponges in summer (78 \pm 15 S.E. sponges $\mathrm{m}^{-2}$ January 2009). In contrast, Breaker Bay and the Near Sirens had their lowest densities of calcareous sponges in winter (Breaker Bay - $17 \pm 3$ S.E. sponges $\mathrm{m}^{-2}$ and Near Sirens $55 \pm 9$ S.E. sponges $\mathrm{m}^{-2}$ in August 2009).

demosponge densities were at their highest in at least one of the autumn sampling periods for all three sites (Breaker Bay $83 \pm 6$ S.E. sponges $\mathrm{m}^{-2}$ May 2008 and $88 \pm 15$ S.E. sponges $\mathrm{m}^{-2}$ in April 09; Far Sirens $171 \pm 30$ S.E. sponges $\mathrm{m}^{-2}$ in May 2008; and Near Sirens $202 \pm 24$ S.E. sponges $\mathrm{m}^{-2}$ in April 2009. All the sites had their lowest densities of demosponges over the sampling period in winter 2009 (Breaker Bay $33 \pm 7$ S.E. sponges m²-2 Near Sirens 21 1 S.E. sponges $\mathrm{m}^{-2}$ ) or spring 2009 (Breaker Bay $28 \pm 3$ S.E. sponges $\mathrm{m}^{-2}$; Far Sirens 87 \pm 6 S.E. sponges $\mathrm{m}^{-2}$; and Near Sirens $48 \pm 5$ S.E. sponges $\mathrm{m}^{-2}$ ).

The results of the MDS plots show that the sponge assemblage composition differs between sites and seasons (Fig. 4.3). The Breaker Bay site showed the most variability between seasons compared with Near Sirens and Far Sirens. The Near Sirens site had the least seasonal variability compared to the other two sites apart from in winter 2009, where the assemblage shifted in August 2009, however, it returned to a similar assemblage as seen previously by November 2009 (spring). The Far Sirens site was intermediate compared to the other two sites in terms of seasonal variability; however, it did not show as large a winter shift (August) in 2009 as the other two sites.

The significant factors for explaining species assemblage shifts using the PERMANOVA model were site, quadrat (nested in site), and the interaction term site*season (Table 4.2). 


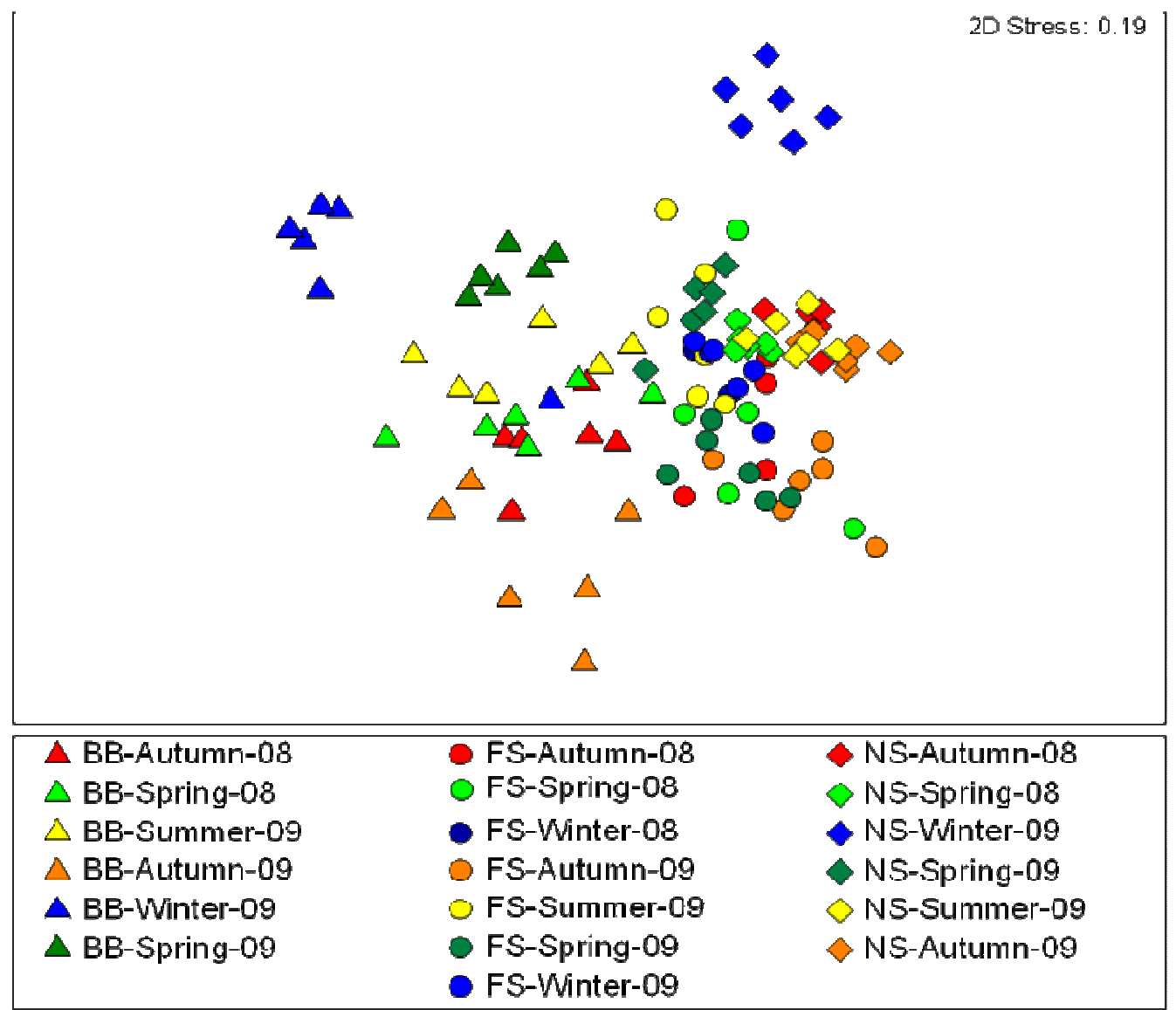

Fig. 4.3 Non-metric multidimensional scaling ordinations of the seasonal changes in sponge density of three sites along the Wellington South Coast. These plots use zero adjusted Bray-Curtis matrices which are $\log (\chi+1)$ transformed from the Far Sirens, Near Sirens and Breaker Bay based on density data.

Table 4.2 PERMANOVA results of the species assemblage data. Significant differences are marked in bold. Unique permutations were observed out of 1000.

\begin{tabular}{|c|c|c|c|c|c|c|}
\hline Source & $\begin{array}{l}\text { Degrees of } \\
\text { freedom }\end{array}$ & $\begin{array}{l}\text { Sums of } \\
\text { squares }\end{array}$ & $\begin{array}{l}\text { Expected mean } \\
\text { of squares }\end{array}$ & $\begin{array}{l}\text { Pseudo- } \\
\text { F }\end{array}$ & $P($ perm $)$ & $\begin{array}{l}\text { Unique } \\
\text { perms }\end{array}$ \\
\hline \multicolumn{7}{|l|}{ Species Assemblage } \\
\hline Year & 1 & 1823.9 & 1823.9 & 1.1607 & 0.322 & 996 \\
\hline Site & 2 & 34170 & 17085 & 6.2548 & 0.001 & 997 \\
\hline Quadrat(Site) & 15 & 16560 & 1104 & 2.4485 & 0.001 & 997 \\
\hline Season(Year) & 5 & 18393 & 3678.6 & 1.4742 & 0.064 & 997 \\
\hline YearSite & 2 & 3770.3 & 1885.2 & & 0.73 & 998 \\
\hline & & & & 0.69016 & & \\
\hline YearQuadrat(Site) & 15 & 6959.1 & 463.94 & 1.0289 & 0.415 & 996 \\
\hline Site*Season $(Y e)^{\star *}$ & 8 & 22844 & 2855.5 & 6.3331 & 0.001 & 999 \\
\hline Residual & 58 & 26152 & 450.89 & & & \\
\hline Total & 106 & 1.4059E5 & & & & \\
\hline
\end{tabular}




\subsubsection{TEMPORAL VARIABILITY OF THE IMPORTANT SPECIES}

SIMPER analysis showed that calcareous sponges were the most important in terms of explaining both the similarities within sites and the differences between sites, as well as seasonal similarities and differences (See Appendix 4.6 for the SIMPER data). When the data were divided by season across all sites, seven to nine species were able to characterise sixty percent of the similarity within a season. The calcareous sponge Clathrina sp. 2. was the most important in density within each of the seasons: $9.63 \%$ in spring, $10.24 \%$ in summer, $10.58 \%$ in autumn and $16.22 \%$ in winter. Twelve to seventeen species were needed to explain sixty percent of the dissimilarity between seasons with the greatest number of species being required to explain the differences between autumn and spring. The seasons with the highest dissimilarity were autumn and winter, which were $51.98 \%$ dissimilar. Autumn and summer were the most similar seasons with $39.01 \%$ dissimilarity.

Seven to eight species were required to explain over $60 \%$ of the similarity between the three sites across all seasons. Clathrina sp. 2 was the most consistently abundant species for the Near Sirens (12.98\%) and the Far Sirens site (15.18\% contribution). For Breaker Bay Clathrina sp. 2 was not important (therefore was not in the $60 \%$ largest contributors of similarity) in order to explain the similarity between Breaker Bay quadrats. For Breaker Bay another calcareous species Sycon sp. was the most important contributor across all seasons (14.11\%). Sycon sp. was not an important species for explaining the similarity within the Near Sirens or Far Sirens sites. Twelve to fourteen species were needed to explain $60 \%$ of the dissimilarity between each of the paired sites. Near Sirens and Far Sirens were the most similar sites with $46.10 \%$ dissimilarity and Breaker Bay and Near Sirens were the least similar sites with $56.09 \%$ dissimilarity. The similarity between the sites over time is similar to that found in the single sampling session in chapter 3. Other important species involved in explaining the similarities and differences between sites were other 
Clathrina spp., Halisarca sp., Oscarella lobularis, Leucosolenia echinata, Plakina c.f. trilopha and Stelletta sp. The seasonal densities of these species are shown in Figs. 4.4-4.11. The most abundant and highly variable species were both from the encrusting calcareous Clathrina genus Figs. 4.4 \& 4.5. Both Clathrina species show similar seasonal fluctuations in densities, (high in autumn and summer and low in spring) and are present in very low densities at Breaker Bay compared to the Near and Far Sirens sites. Generally, for all three sites and eight species there was a decrease in the density of the species in spring 2009 and generally higher densities in autumn and summer. The exceptions would be for the calcareous species Sycon sp. and Leucosolenia echinata at Breaker Bay, which increased in abundance in spring 2009; however, it is important to note that the increase was insignificant due the both species being less common than all the other species included here (Figs. $4.6 \& 4.7$ ). Leucosolenia echinata at the Far Sirens and Breaker Bay and Sycon sp. at all three sites decreased to a density of less the 5 sponges per $\mathrm{m}^{-2}$ in autumn 2009, which is the opposite pattern to the demosponges (Figs. $4.6 \& 4.7$ ).

Oscarella lobularis, Halisarca sp. and Plakina c.f. trilopha are all encrusting ephemeral demosponges that form small patches on the bedrock and sometimes other sponges and sessile benthos. They were very variable in density in particular at the Far Sirens site (Figs. 4.8-10). Oscarella lobularis, whose abundance is shown in Fig. 4.8, tended to decrease over the study period at the Far Sirens and particularly decrease in winter 2009 and continue to have low abundance in spring 2009. Halisarca sp. had its highest abundances in both autumn sampling periods and the lowest densities in winter and spring 2009 for the Far and Near Sirens and remained at low densities for Breaker Bay throughout the sampling period (Fig. 4.9). Plakina c.f. trilopha reached its highest densities in the autumn sampling periods for Far and Near Sirens but was only present in low densities at Breaker Bay for the entire sampling period (Fig. 4.10). 
Chapter 4 Temporal variability in sponge assemblages on the Wellington South Coast, New Zealand

Stelletta sp. is a massive demosponge that often has sponges, ascidians and calcareous algae growing on its surface. The densities of Stelletta sp. with the mean densities for all sites remaining around 5-15 sponges per $\mathrm{m}^{-2}$ apart from in March 2009 in the Far Sirens when densities increased to about 17 with a large S.E. value because of the high within site variability (Fig. 4.11). 
Chapter 4 Temporal variability in sponge assemblages on the Wellington South Coast, New Zealand

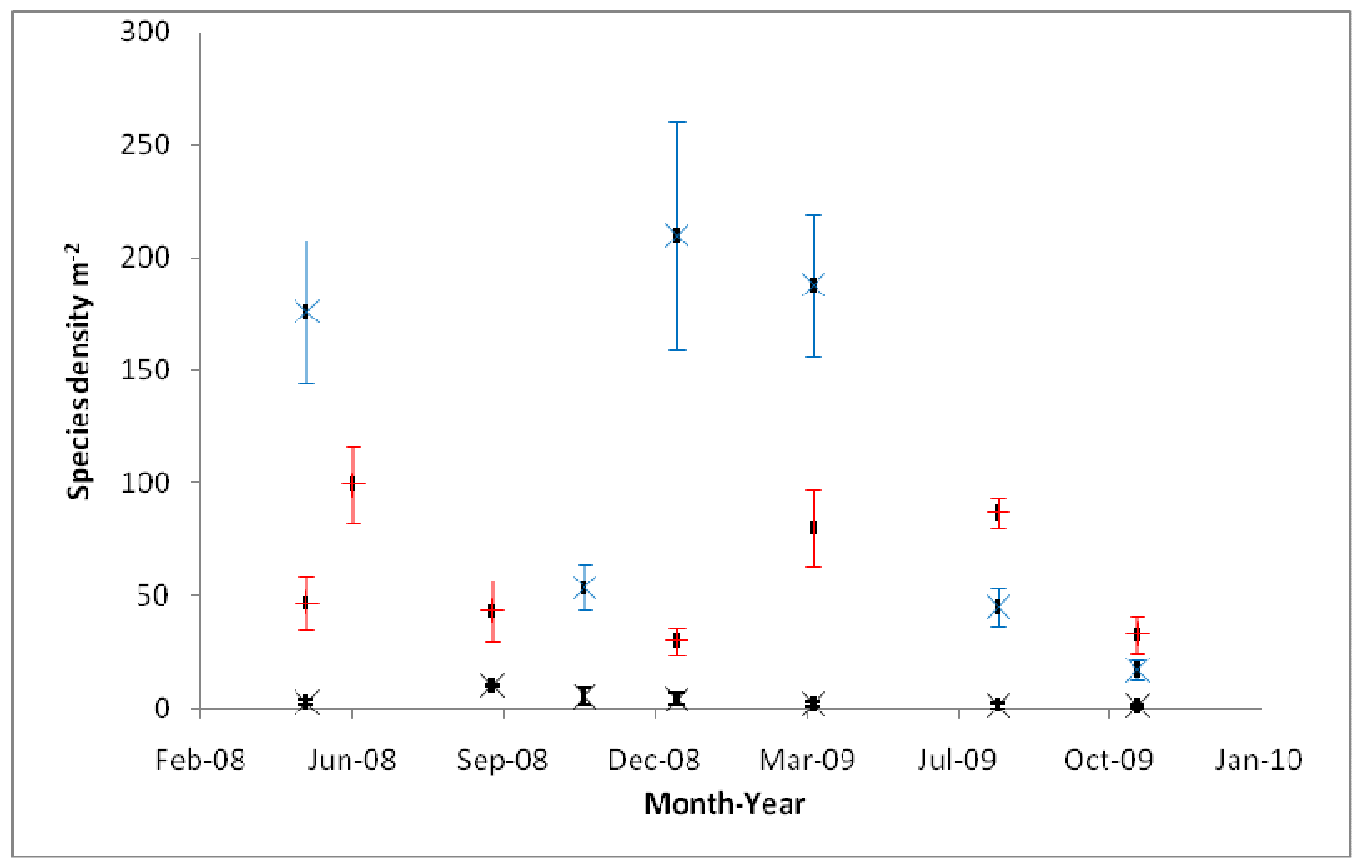

Fig. 4.4 The seasonal densities and S.E. bars for Clathrina sp.2 from Breaker Bay (x) Far Sirens (+) and Near Sirens $(x)$.

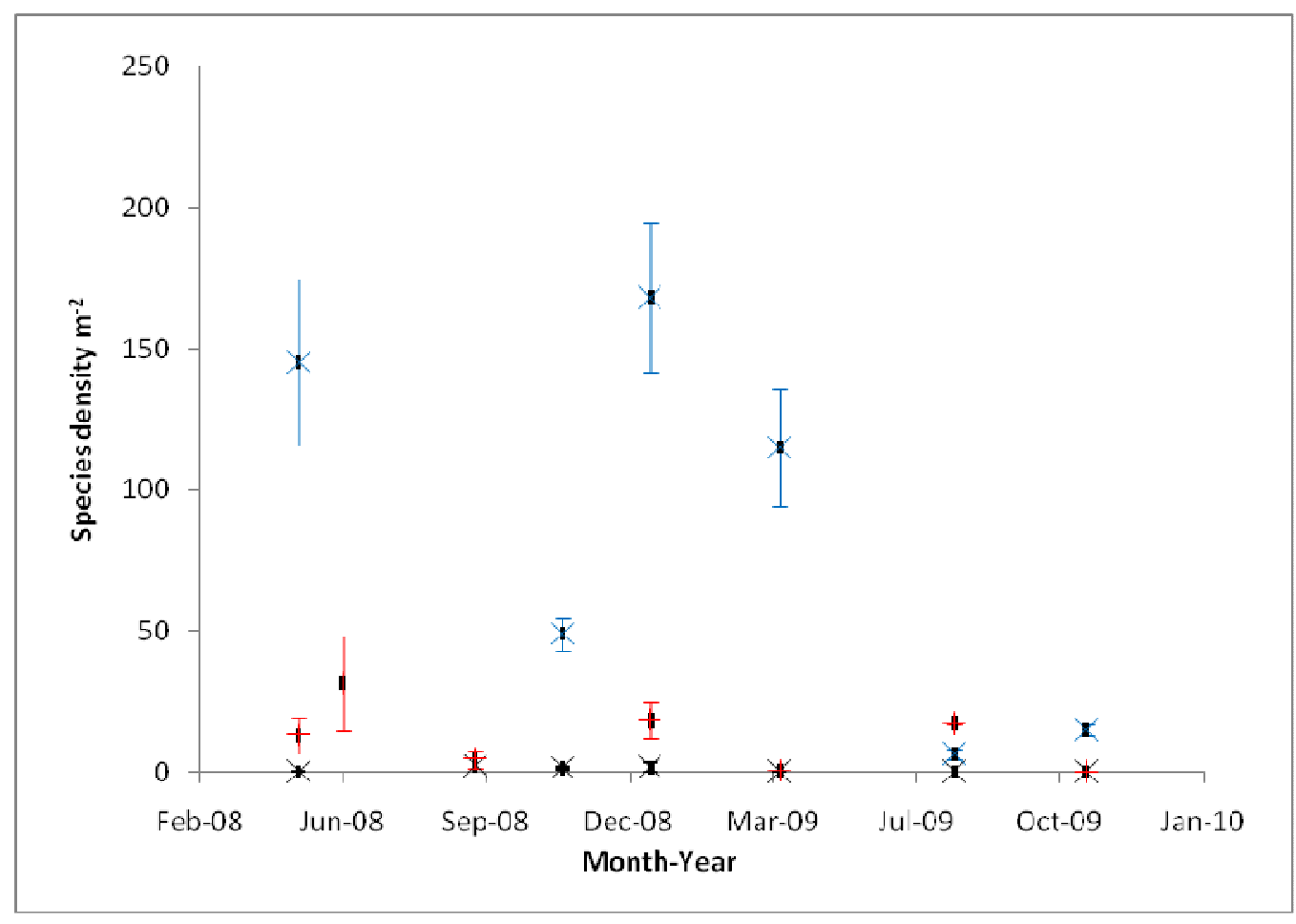

Fig. 4.5 The seasonal densities and S.E. bars for Clathrina sp.3 from Breaker Bay (x) Far Sirens (+) and Near Sirens $(x)$. 
Chapter 4 Temporal variability in sponge assemblages on the Wellington South Coast, New Zealand

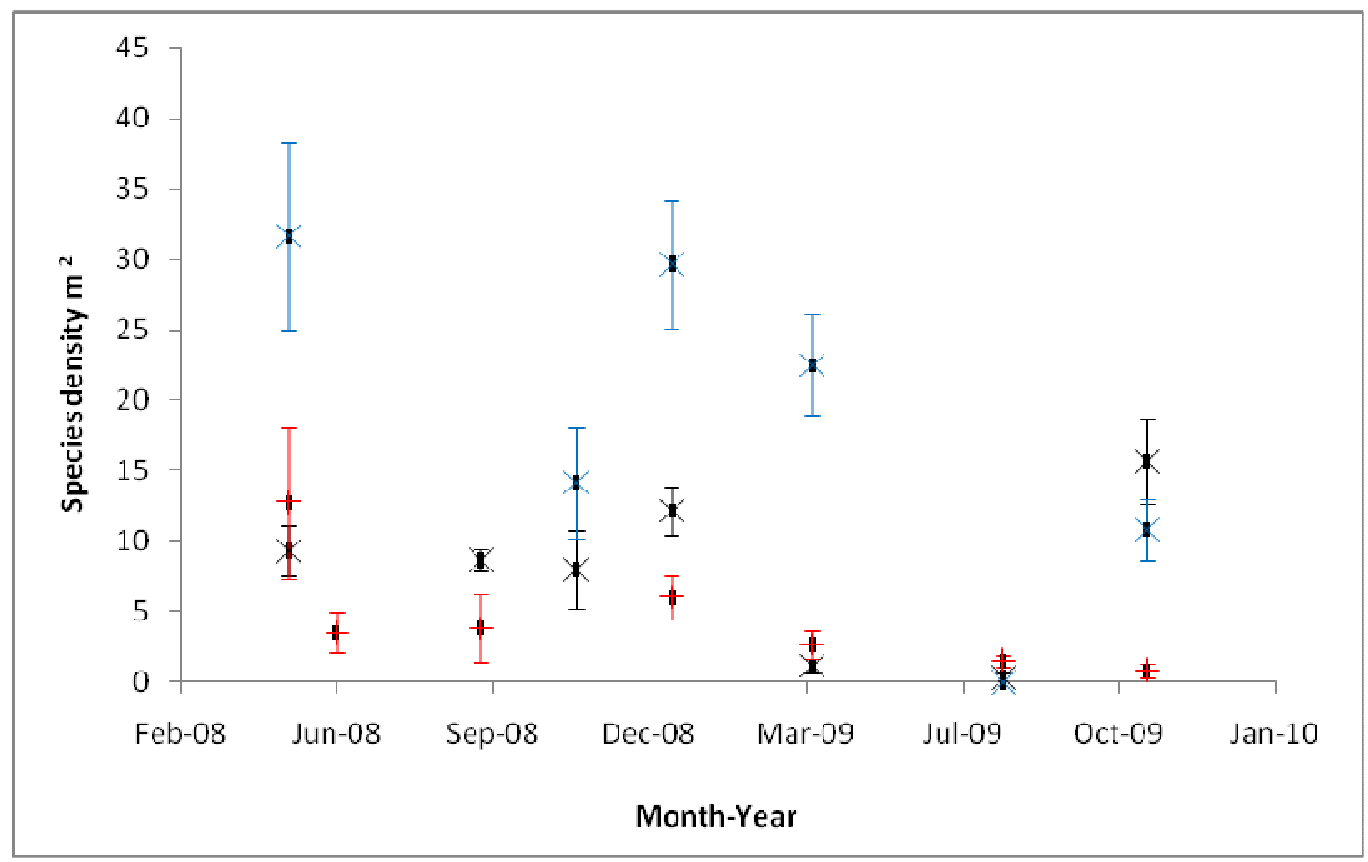

Fig 4.6 The seasonal densities and S.E. bars for Leucosolenia echinata from Breaker Bay (x) Far Sirens (+) and Near Sirens (x).

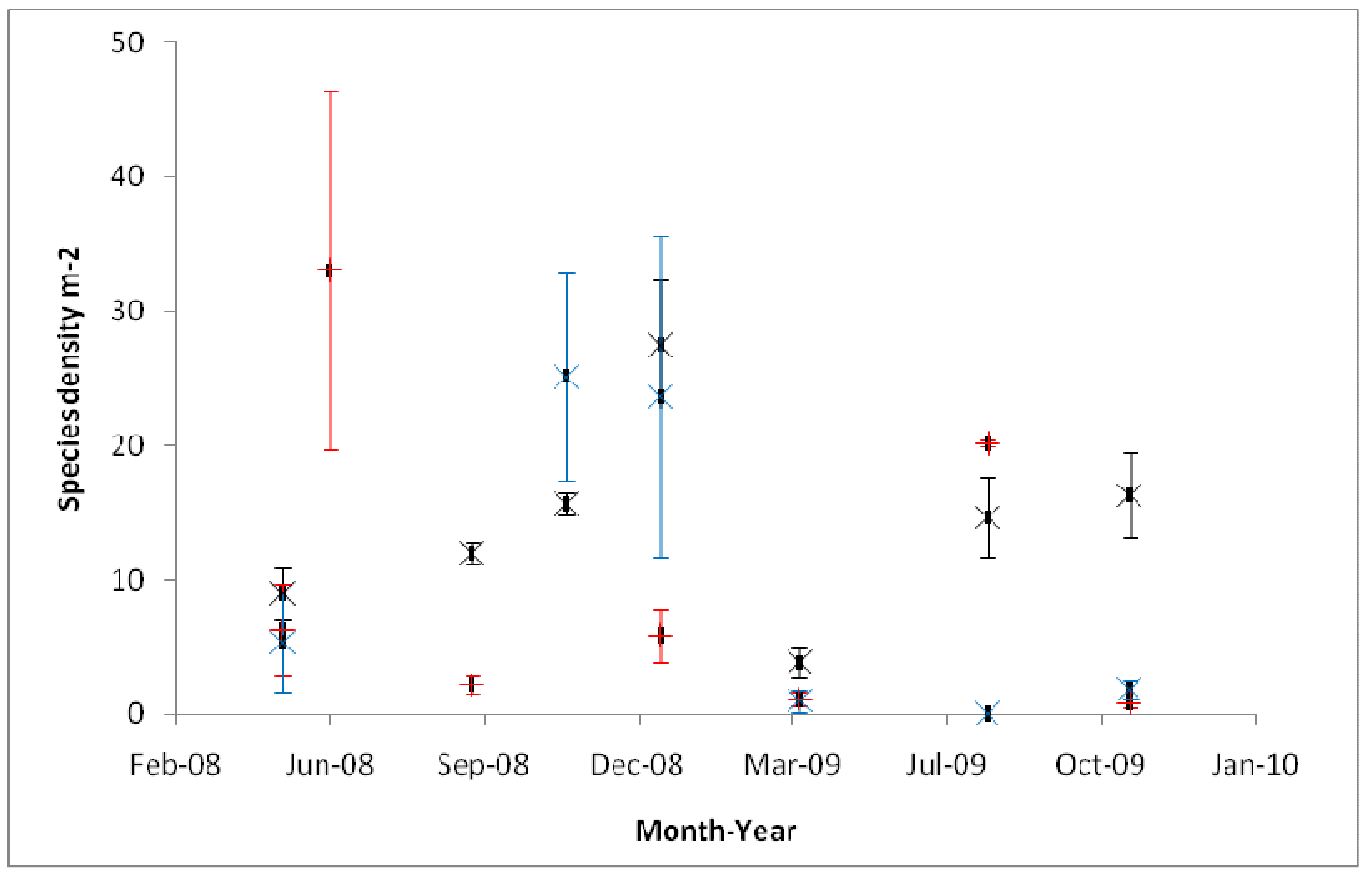

Fig 4.7 The seasonal densities and S.E. bars for Sycon sp. from Breaker Bay (x) Far Sirens (+) and Near Sirens (x). 
Chapter 4 Temporal variability in sponge assemblages on the Wellington South Coast, New Zealand

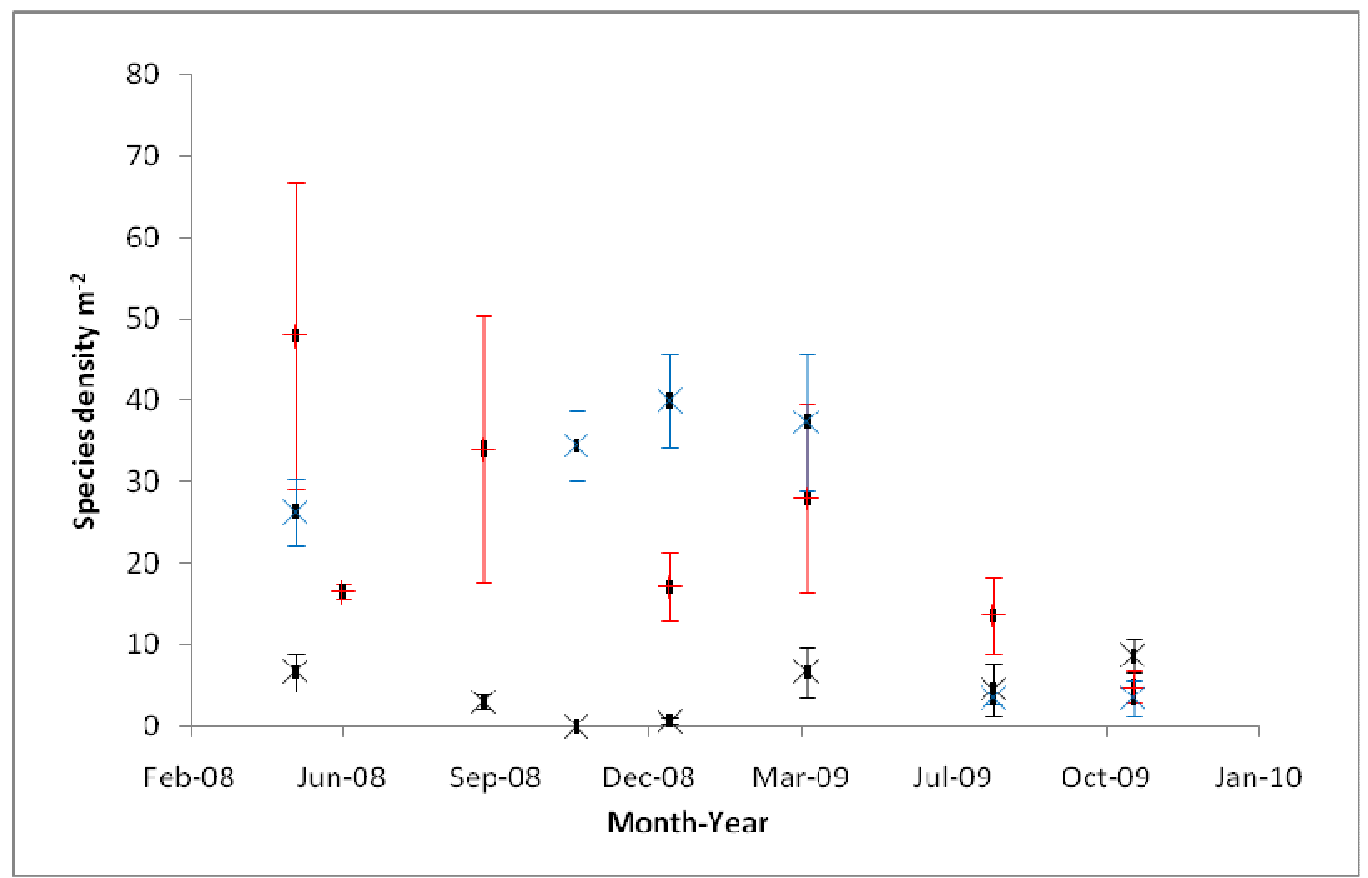

Fig 4.8 The seasonal densities and S.E. bars for Oscarella lobularis from Breaker Bay (x) Far Sirens $(+)$ and Near Sirens (x).

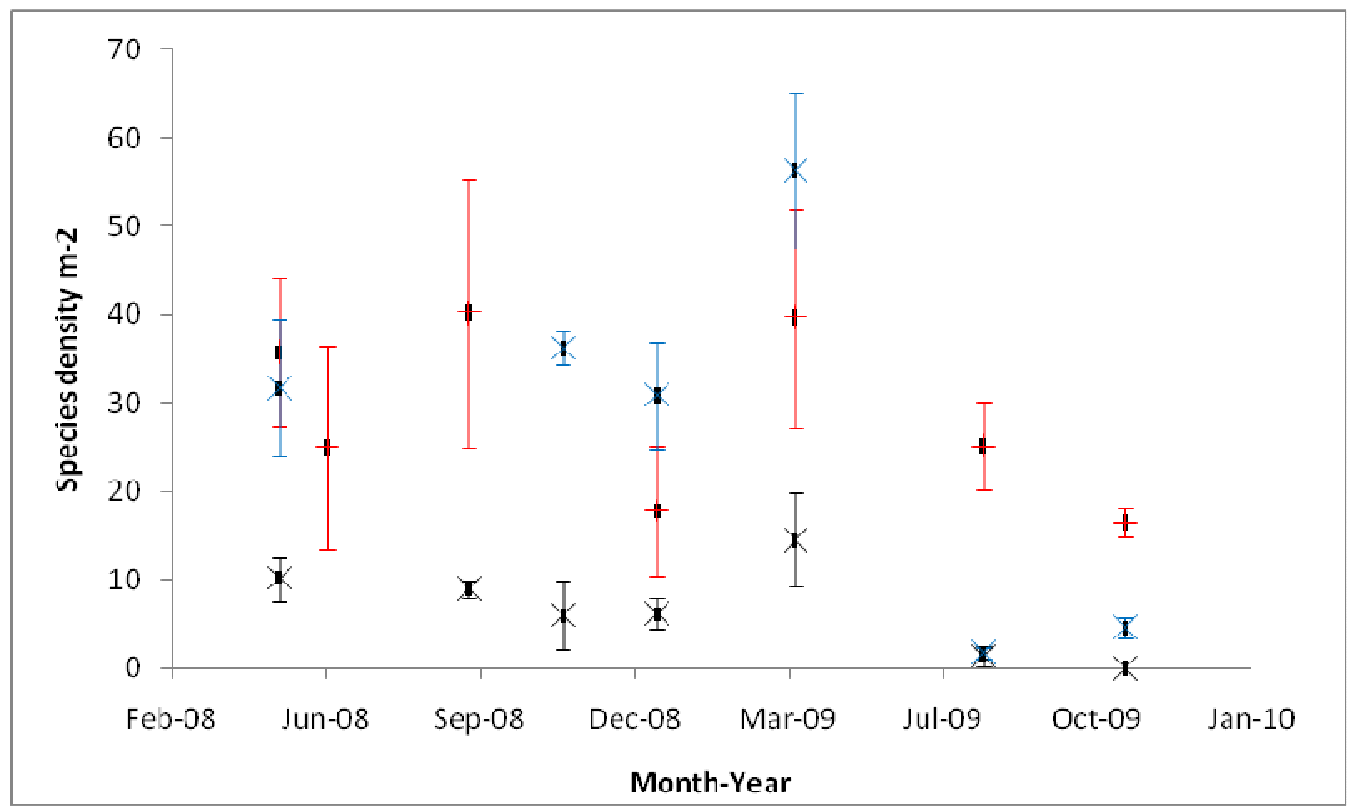

Fig 4.9 The seasonal densities and S.E. bars for Halisarca sp. from Breaker Bay (x) Far Sirens $(+)$ and Near Sirens ( $\mathrm{x})$. 
Chapter 4 Temporal variability in sponge assemblages on the Wellington South Coast, New Zealand

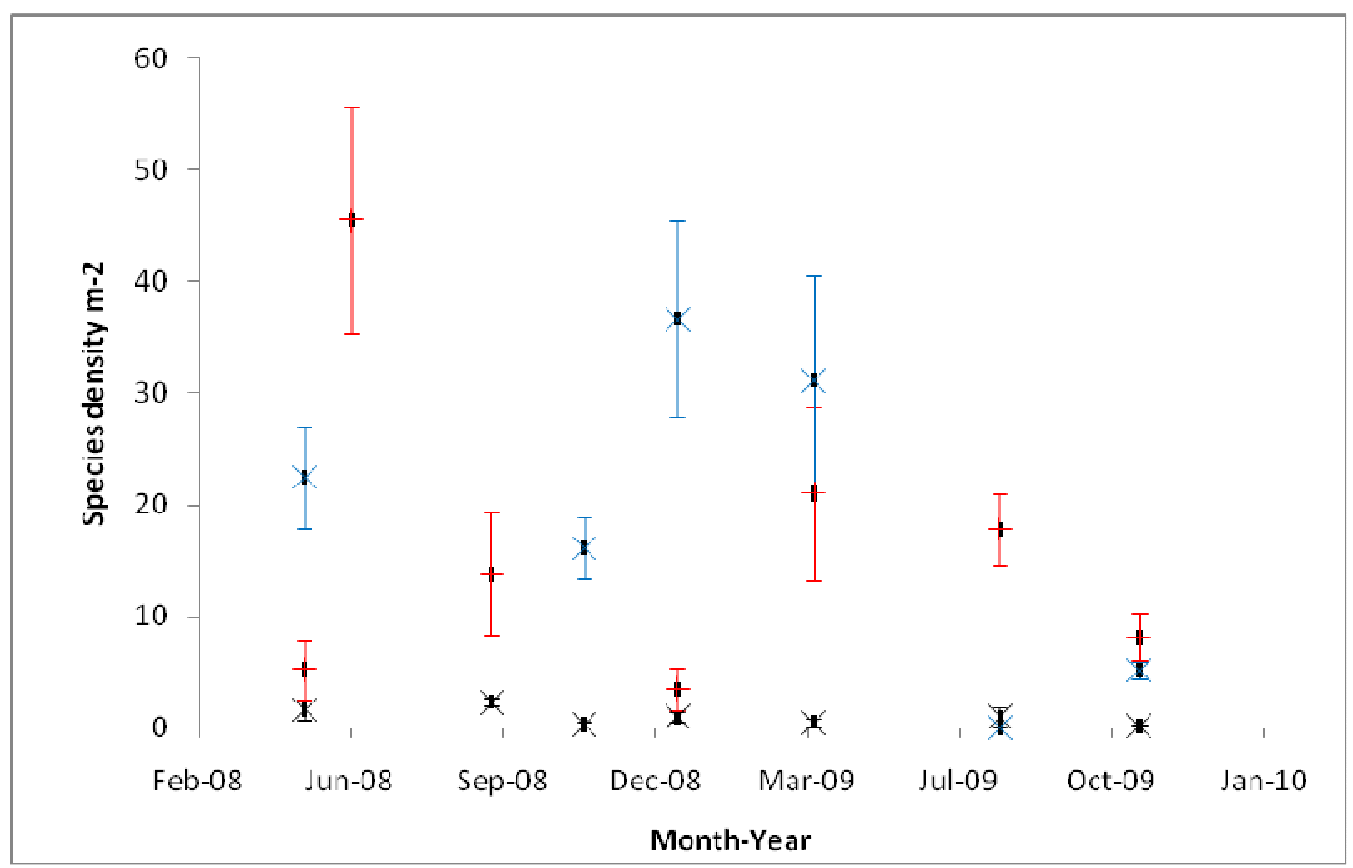

Fig 4.10 The seasonal densities and S.E. bars for Plakina c.f. trilopha from Breaker Bay (x) Far Sirens (+) and Near Sirens (x).

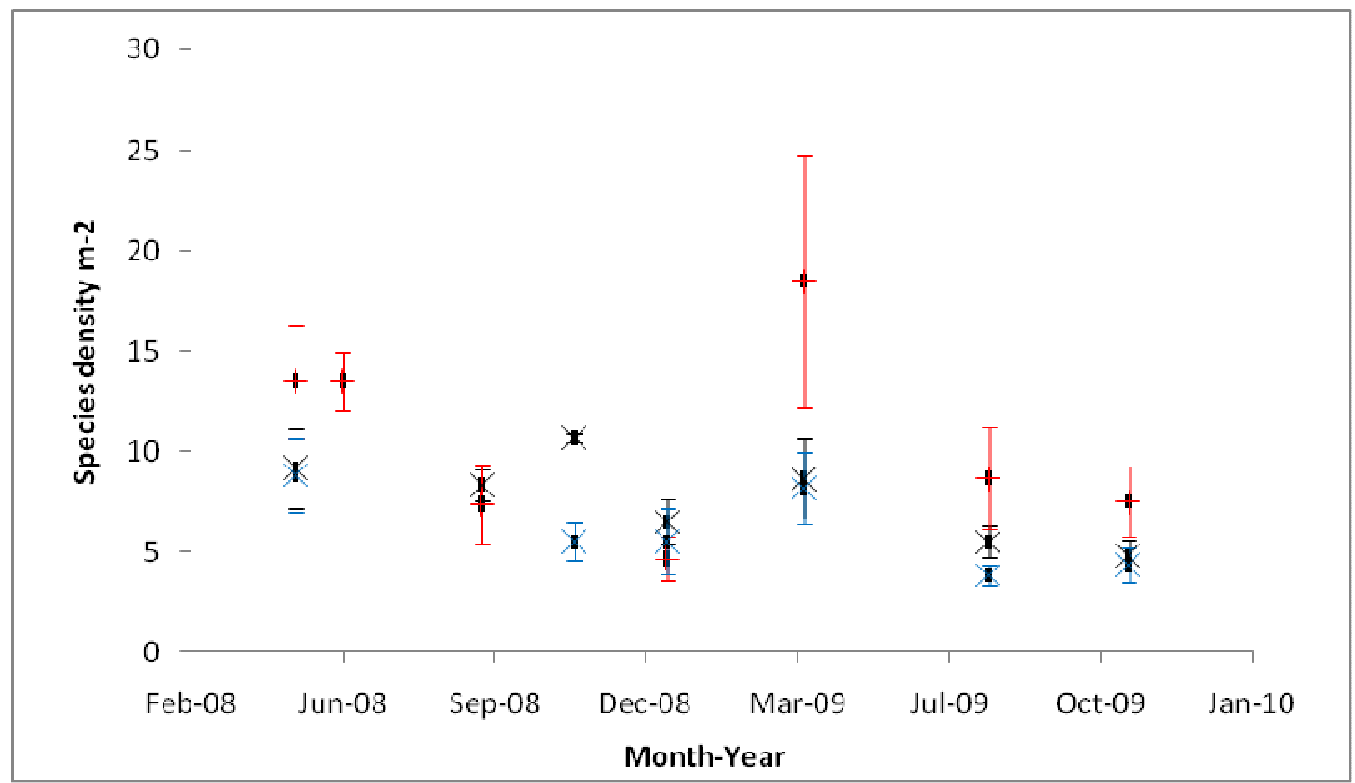

Fig 4.11 The seasonal densities and S.E. bars for Stelletta sp. from Breaker Bay (x) Far Sirens (+) and Near Sirens (x). 


\subsubsection{ENVIRONMENTAL DATA}

Using the DistLM function in PRIMER with 999 permutations and the BEST function, all of the environmental variables measured by the NIWA Wellington airport meteorological station from January 2007 till November 2009 and all the available seawater temperature data were significantly correlated with the patterns of sponge abundance which included: total monthly rainfall $(\mathrm{F}=2.43$ $\mathrm{P}=0.021)$, highest speed wind gust $(\mathrm{F}=1.99 \mathrm{P}=0.048)$, mean monthly air temp $(\mathrm{F}=4.60 \mathrm{P}=0.001)$ and mean air pressure at 9am $(\mathrm{F}=4.60 \mathrm{P}=0.001)$ and seawater temperature $(F=6.20$ and $p=0.001)$. The best single environmental factor correlation was the seawater temperature data which had an AICc value of 766.32 $\mathrm{R}^{2}=0.056$. The best solution from the variables used the seawater temperature and the rainfall data and had a AICc value of $765.36 \mathrm{R}^{2}=0.083$. These two variables explain about $8 \%$ of the variation in sponge diversity and abundance.

The seawater temperatures were measured between the 21st June 2008 and the $23^{\text {rd }}$ May 2009. The mean temperatures for the readings each day at $9 \mathrm{am}$ across each month are shown in fig. 4.12. The Far Sirens temperatures were generally slightly $\left(<0.1^{\circ} \mathrm{C}\right)$ cooler than the Near Sirens temperatures. The highest temperatures recorded were on the $28^{\text {th }}$ April 2009 at the $4 \mathrm{pm}$ and $4.30 \mathrm{pm}$ reading at the Near Sirens $\left(23.48^{\circ} \mathrm{C}\right)$ and at $5 \mathrm{pm}$ at the Far Sirens $\left(23.39^{\circ} \mathrm{C}\right)$. The lowest seawater temperatures for the Near and Far Sirens sites were on the $18^{\text {th }}$ August 2009 between 7:30am and 9:30am and these were $10.65^{\circ} \mathrm{C}$ at the Far Sirens and $10.55^{\circ} \mathrm{C}$ at the Near Sirens sites. The mean monthly water temperatures were coldest in August 2008 and warmest in March 2009. The water temperature in January, February April and May 2009 were all very similar around $15.1^{\circ} \mathrm{C}$. This stability in the seawater temperature in summer and autumn 2009 is not shown in the air temperature data shown in Fig. 4.13. 
Chapter 4 Temporal variability in sponge assemblages on the Wellington South Coast, New Zealand

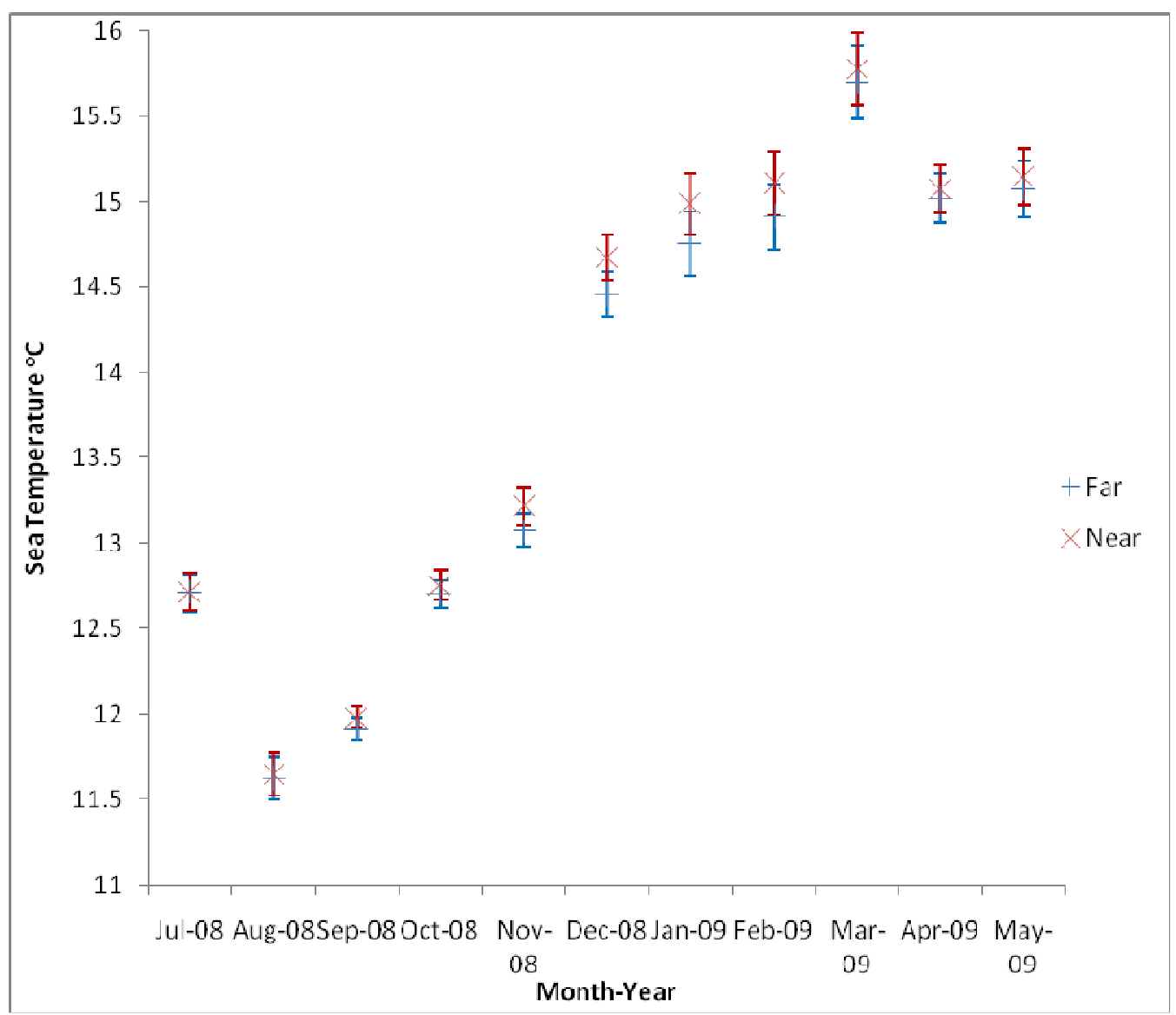

Fig. 4.12 The mean seawater temperature in ${ }^{\circ} \mathrm{C} \pm$ S.E. for the Near Sirens and Far Sirens sites from July 2008 - May 2009 at 9am.

The air temperature data showed clear seasonal patterns and is shown in Fig. 4.13. The coolest months during the study were June and July 2009, which had mean temperatures of $8.8^{\circ} \mathrm{C}$. The two warmest months were January and February 2008 which had mean monthly air temperatures of $18.8^{\circ} \mathrm{C}$ and $18.6^{\circ} \mathrm{C}$ respectively. 
Chapter 4 Temporal variability in sponge assemblages on the Wellington South Coast, New Zealand

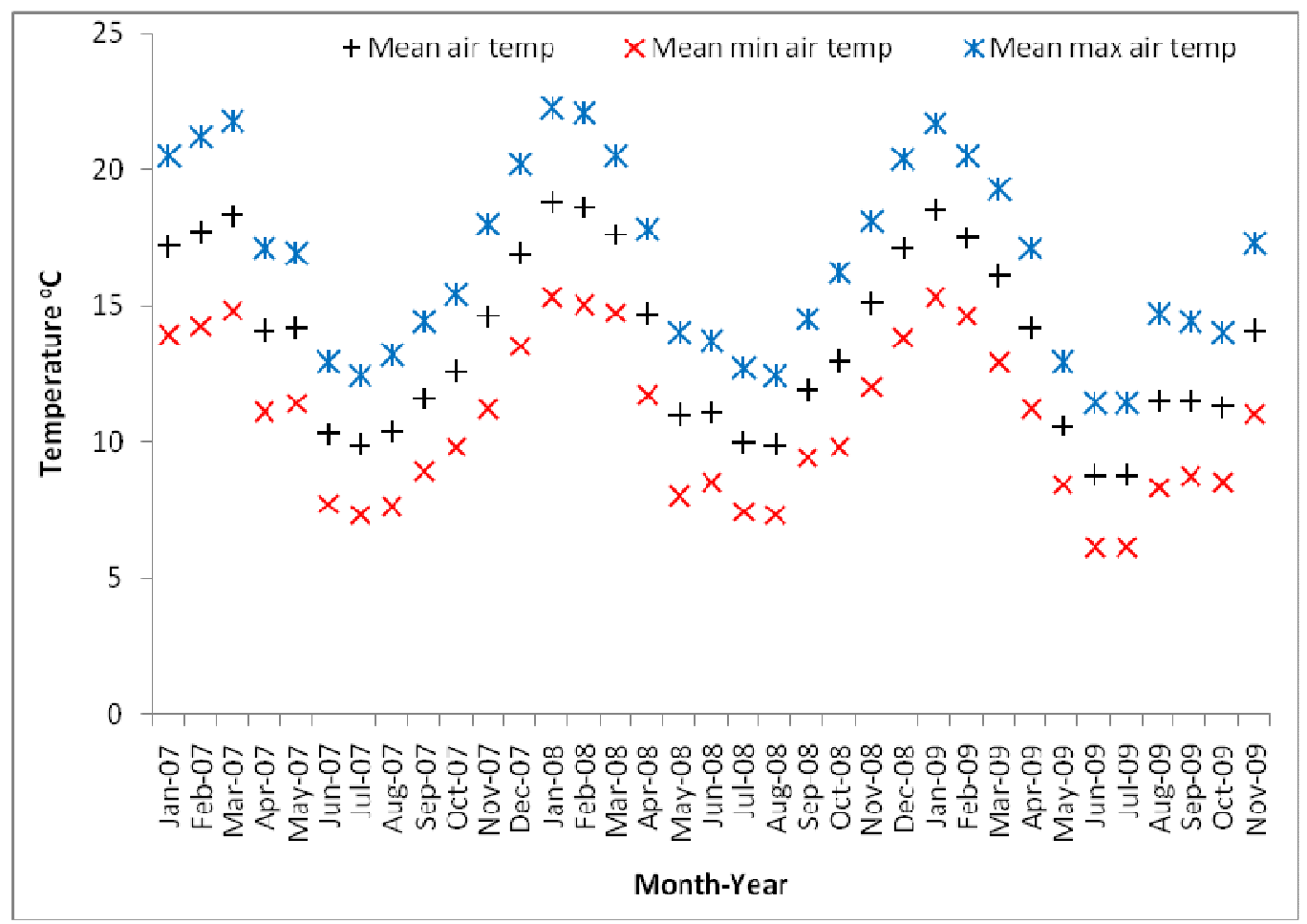

Fig. 4.13 Monthly mean air temperatures for the Wellington South Coast. The monthly mean and the lowest and highest temperature daily means within each month are shown here. Data are taken from the NIWA meteorological station at Wellington airport on the south coast of Wellington, New Zealand (NIWA, 2011).

The highest monthly wind gust speed data from the NIWA Wellington airport meteorological station from January 2007 till November 2009 is shown in Fig. 4.14. The month with the lowest high wind speed was February 2008 with 20.6 $\mathrm{m} / \mathrm{s}$ while May 2009 had the highest wind gust speed $32.4 \mathrm{~m} / \mathrm{s}$. There are no clear seasonal trends from the wind data although the winds are generally stronger in autumn and winter and lower in spring and summer. 


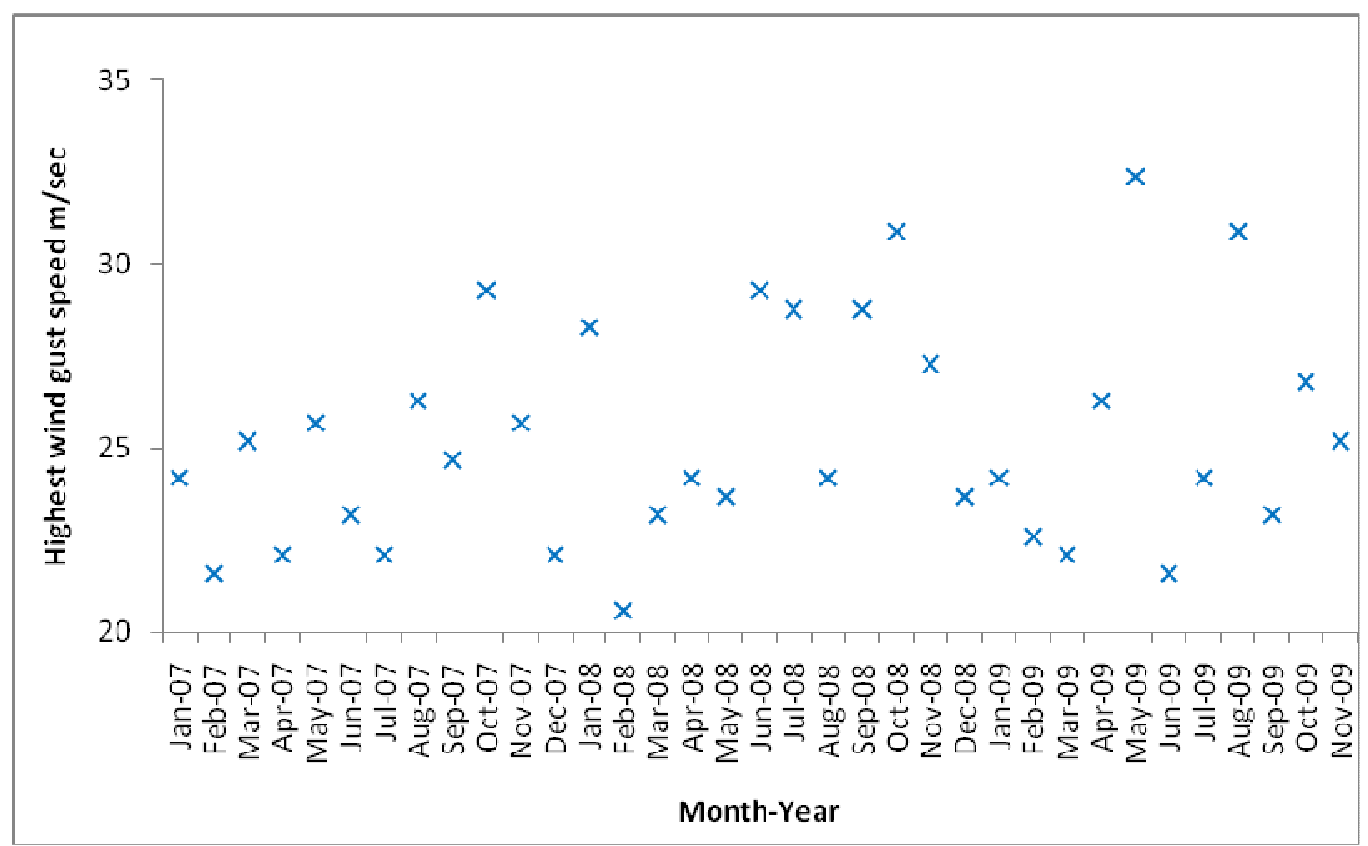

Fig. 4.14 Monthly highest wind gust speeds for the Wellington South Coast. Data are taken from the NIWA meteorological station at Wellington airport on the south coast of Wellington, New Zealand (NIWA, 2011).

The total monthly rainfall data from the NIWA Wellington airport meteorological station from January 2007 till November 2009 is shown in Fig. 4.15. The month with the most rain was July 2008 with $192.8 \mathrm{~mm}^{3}$ while the driest month was January 2009 with $14.6 \mathrm{~mm}$ of rain. Generally the summers are drier than the winters however there is no clear trend from the rain data.

The mean monthly air pressure data from the NIWA Wellington airport meteorological station from January 2007 till November 2009 is shown in Fig. 4.16. The month with the highest mean pressure was April 2009 with 1022.3HPa and the lowest pressure was August 2008 with 1008.7HPa. There is no clear seasonal trend in air pressure from the data. 
Chapter 4 Temporal variability in sponge assemblages on the Wellington South Coast, New Zealand

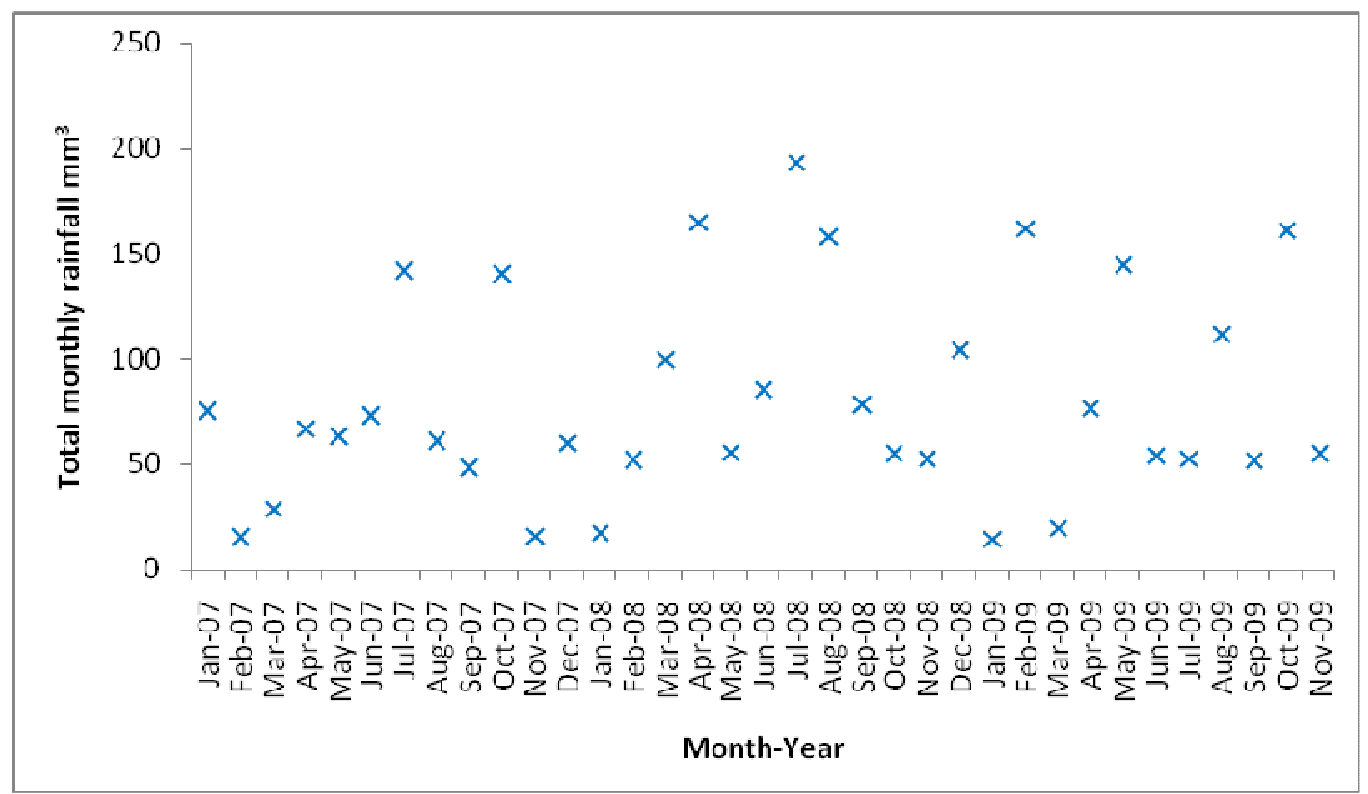

Fig. 4.15 Total monthly rainfall for the Wellington South Coast. Data are taken from the NIWA meteorological station at the airport on the south coast of Wellington, New Zealand (NIWA, 2011).

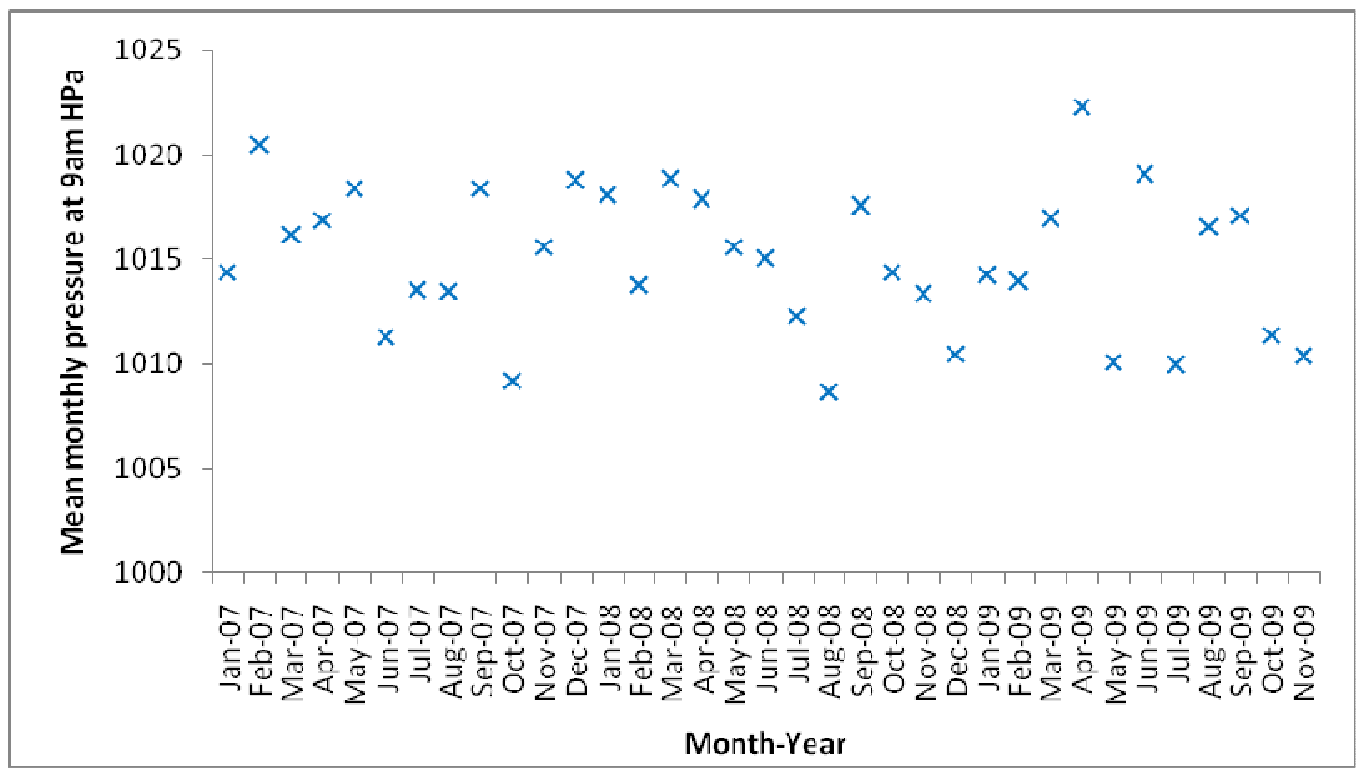

Fig. 4.16 Mean monthly air pressure for the Wellington South Coast. Data are taken from the NIWA meteorological station at the airport on the south coast of Wellington, New Zealand (NIWA, 2011). 


\subsection{DISCUSSION}

\subsubsection{TEMPORAL VARIABILITY OF THE ASSEMBLAGES}

This work provides the first quantitative survey of the temporal variation of the sponge assemblages on the Wellington South Coast, New Zealand. The multi and univariate data showed that there were significant site and the interaction between site and season differences in the sponge assemblages on the South coast and season had a weakly insignificant effect. The interaction between site and season means that each site changes differently in relation to season and it is not possible to isolate the effect of either site or season completely, and that each of the sites responded in a different way to seasonal changes. The species richness was significantly different between seasons and the interaction between season and site was also significant therefore there was a shift in sponge diversity between seasons. Total sponge density was also influenced by the interaction between site and season and there were also significant site differences.

The sites were initially chosen as they appeared to be similar to each other (similar depths and substrata) and to represent similar biodiverse sponges assemblages along the Wellington South coast. However, from chapter 3 and this chapter it can be seen that there are significant differences between the sites. There is an east to west gradient in macrobenthic communities along the Wellington South Coast from the mouth of the harbour (where Breaker Bay is close to the edge of) to Island Bay. Island Bay is on the high energy very exposed Wellington South Coast. Therefore the significant differences between the Sirens sites could be expected, even though the Sirens sites are about the same difference away from the harbour. The Sirens sites are only $50 \mathrm{~m}$ apart while Breaker Bay is about 6km away from the harbour mouth (Helson et al., 2007; Pande \& Gardner, 2009). For the Wellington South coast there is a range of fish, invertebrates and algae that have been found to have seasonal patterns of abundance including the banded wrasse, blue cod, yellow foot paua and the 
macroalgae Carpophyllum maschalocarpum and Ecklonia radiata (Pande \& Gardner, 2009). However, in the same study there are also fish (butterfish and tarakihi), algae (Lessonia variegata) and invertebrates (black foot paua, rock lobster and kina) that showed no evidence of variation in seasonal abundance over a three year study (Pande, 2001; Pande \& Gardner, 2009).

All sites had higher between site variability than within site variability according to the SIMPER analysis. Breaker Bay had the highest within site variability, while the Near Sirens site had the lowest within site variability. Both of the Siren's sites were also more similar to each other than to the Breaker Bay site and therefore shared similar species compositions and densities through the seasons.

Other studies of sponge assemblage spatial variation in temperate waters have also shown considerable spatial variation between locations (Kennelly \& Underwood, 1992; Roberts \& Davis, 1996). The factors likely to be responsible for this spatial variation could also influence the temporal variation including larval supply, recruitment, physical disturbance and biological interactions (Underwood et al., 1991). Abiotic factors are thought to be the main drivers of changes in temperate sponge assemblages. (Bell \& Barnes, 2000b, c). The Wellington South coast is a very active hydrodynamic area and variation in the energy reaching the sites and scouring away near shore sponge assemblages is likely to be high. There were often new bare patches in the quadrats which could have been caused by extreme weather events. Other reasons could be that all three sites are popular recreational dive sites and as they are narrow channels in a dynamic environment there could be damage to the sponges on the walls through accidental diver contact. In a study on divers in the Mediterranean over 96\% made contact with the seabed when diving (Luna et al., 2009) and there is likely to be more contact in the channels of Wellingtons South coast due to Wellingtons weather conditions as it is rarely flat calm (Pande, 2001). 
Other biological reasons for temporal variation in sponge species could be due the naturally patchy distribution of sponge species and their short range dispersal patterns (Mariani et al., 2006). One example of a calcareous sponge clustering is Sycon sp., which is a small vase shaped sponge that can be found attached to the bedrock or frequently on the surface of the massive Stelletta sp. sponge. Often there were either hundreds of Sycon sp. of a similar size, which is likely to be due to be a single recruitment event all clustered within tens of centimeters' on one sponge, while another Stelletta sp. close by will have just one or two Sycon sp.

Another potential biological factor that could be responsible for temporal differences could be predation. For example, spongivorous fish or nudibranchs, as well as the mechanisms used by sponges to deter predators, can play a role in shaping sponge assemblage structure on temperate reefs although at it has been reported at a lower intensity than in tropical areas (Ruzicka \& Gleason, 2008; Ruzicka \& Gleason, 2009). There is no information on spongivorous fish along the Wellington South Coast. However, leatherjackets (Parika scaber) are a fish species known to utilize encrusting animals as a major part of their normal diet, including a sponges as a dominant food item, which has been observed during the survey dives (Russell, 1983). The seasonal abundance of leatherjackets in Wellington waters is not known however they settle in higher abundances in the summer months compared to the winter in the Leigh Marine Reserve (Kingsford \& Milicich, 1987). Blue eyed triple fins (Notoclinops segmentatus) and the yellow and black triplefin Fosterygion flavonigrum species, which are found along the Wellington south coast are known to eat sponges as a significant part of their diet (Russell, 1983). Blue Cod (Parapercis colias) have also been observed occasionally biting off small chunks of unknown sponges and spicules have been found in gut content analysis in Fiordland (N. Beer pers. com.). Blue cod adapt their diet to available food sources and eat a wide range of prey and non-prey items (Rodgers \& Wing, 2008). Blue cod numbers are known to have increased within the Taputeranga Marine Reserve 
since it was established in 2008 (T. Eddy pers. com.), which appears to be typical for marine reserves in New Zealand (Pande et al., 2008). Therefore if fish numbers continue to increase as predicted within the marine reserve (Pande et al., 2008) there is potential for sponge species to decrease in abundance, although sponges are probably an accidental food item for blue cod rather than an important prey item (N. Beer pers. com.).

Other potential sponge predators include nudibranches or sea slugs which are known to feed, often exclusively, on sponges (Avila \& Paul, 1997; Becerro et al., 1998; Knowlton \& Highsmith, 2005; Page et al., 2010). Many seaslugs have an annual life cycle on the Wellington South coast and therefore are not present in the winter months (Beu \& Marshall, 2008). The nudibranch Hoplodoris nodulosa is found all over New Zealand and it is the first nudibranch recorded to decimate through predation a monoculture (aquaculture) of sponges in the Pelorus Sound (Page et al., 2010). Therefore potentially in areas with high sponge density predation could reduce sponge densities, however, this is unlikely on the Wellington South coast as the high densities of sponges are of a wide range of species and therefore it is not a monoculture and not as vulnerable. There are many other nudibranch species found along the Wellington South coast, however, their specific diets and habitats are not known.

The sponge species found to be important using the SIMPER analysis generally had the highest densities in summer and autumn and lower densities in winter and spring. There were important calcareous and demosponges influencing the overall assemblage patterns. The seawater data showed that the water remained warm $\left(15^{\circ} \mathrm{C}+\right)$ from January through to May 2009 , which could be due to the ending of the La Nina year. Conversely, the coolest months during the study were June and July 2009 when the mean air temperatures dropped to $8.8^{\circ} \mathrm{C}$. The drop in temperature could have led to the die back or shrinkage of some of the species in the winter 2009. The photographs of the permanent 
quadrats were almost unrecognisable as being the same quadrat, in particular at the Near Sirens site where the total sponge abundance dropped from April 2009 from $689 \pm 71$ S.E. sponges $\mathrm{m}^{-2}$ to $76 \pm 9$ S.E. sponges $\mathrm{m}^{-2}$ in August 2009. Many of the species declined between autumn and winter 2009, however, the density of the Clathrina spp. had the largest shift to lower densities at the Near Sirens. However, the Clathrina spp. did not decline in densities from autumn to winter 2009 at the Far Sirens site, which is only $200 \mathrm{~m}$ away.

The temperature differences between the two sites at the Far Sirens were only up to $0.1^{\circ} \mathrm{C}$ cooler than the Near Sirens over the study period and there were no significant seasonal or spatial correlations for a range of nutrients found in a study on the utilisation of nutrients by sponge assemblages carried out concurrently along the Wellington South coast (nitrate, nitrite, ammonium, phosphate and silicate) ((Perea-Blázquez, 2011). The Far Sirens site is more sheltered from the initial force of the swell compared to the Near Sirens as it is in a wider channel parallel to the shore.

\subsubsection{TEMPORAL VARIABILITY OF THE IMPORTANT SPECIES}

The main species explaining the sponge assemblage seasonal and site differences were encrusting to cushion shaped calcareous species, including Clathrina spp. and Leucosolenia echinata, and encrusting small demosponges including Halisarca sp., Oscarella lobularis, and Plakina c.f. trilopha. Encrusting sponges are known to undergo fission and fusion over time, which would increase and decrease the density of sponge patches (Ayling, 1983b; Pansini \& Pronzato, 1985; Tanaka, 2002; Blanquer et al., 2009). Fusion normally only happens between fragments that are not only the same species but are also genetically identical and it was rare in the Wellington South coast quadrats (van de Vyver, 1975; Amano, 1990; Blanquer et al., 2009). The fission and fusion of encrusting sponges has been related to random events (Ayling, 1983b; Tanaka, 2002) and/or seasonality (Blanquer et al., 2008). There were fission events in 
the Wellington South quadrats, however, it tended to be encrusting species that formed large patches, such as Crella sp. and Haliclona sp., which were both unimportant in explaining seasonal changes in the assemblage.

Encrusting sponges have a lower profile and therefore were unlikely to be removed easily when subjected to forces or damaged as easily as massive or arborescent species by storms, however, the loosely attached cushion forms of the Clathrina spp. and Leucosolenia echinata, can easily be removed from vertical walls with the slightest touch and they might have low regeneration rates as no tissue is left behind to support the process (Wulff, 2006b).

Calcareous sponges are thought to be short-lived (less than one year in some cases compared to up to hundreds of years for demosponges) (Reiswig, 1973; Tuzet, 1973; Johnson, 1979). The calcareous sponges in the Wellington South Coast quadrats lived less than a year although they did not all die off at the same time of the year (pers. obs). Clathrina clathrus from the Mediterranean has been shown to have a hypo-active phase over winter allowing it to control its metabolism and maintain biochemical homeostasis over the winter months so potentially this could be possible in other Clathrina spp. (Burlando et al., 1992).

Calcareous sponge species have been reported to increase in abundance and percentage cover in winter, with a die-off in the summer in other temperate locations, which is the opposite pattern reported for many demosponges (Dendy, 1914; Orton, 1914; Burton, 1949; Johnson, 1979). At the Far Sirens site the density of calcareous sponges were at their most abundant in August 2009 and similar numbers were present in the June 2008 (winter). However, at Breaker Bay and the Near Sirens significant decreases in the calcareous sponge density occurred during August 2009 as well as the demosponges. Therefore the different sites have differing seasonal patterns of change in the sponge assemblages.

Stelletta sp. was also a important demosponge for explaining the shifts in sponge assemblages within and between sites and seasons. Stelletta sp. is a 
cushion to massive sponge that can grow to cover areas over $0.5 \mathrm{~m}^{2}$, however, it is a relatively cryptic species as it often has other sponges, calcareous algae and other benthic organisms growing on it. Therefore it can be difficult to see the boundaries between these sponges. Therefore the variability of the abundance of this species can be difficult to count in situ or from a photo, however, it remained relatively stable in abundance throughout the study period. Stelletta $s p$. can reproduce by budding increasing their density in the local area around and on the surface of the adult sponge which is what happened in the Far Sirens in March 2009 (Fell, 1993). All of the extra young Stelletta sp. sponges had disappeared by August 2009 as they were likely to still have been loosely attached and swept away by the surge.

\subsubsection{ENVIRONMENTAL DATA}

All of the environmental variables measured significantly correlated with the patterns of the sponge assemblage data which included: total monthly rainfall highest speed wind gust, mean monthly air temp, mean air pressure at 9am and mean seawater temperature at 9am. The best single environmental factor correlation was the seawater temperature data although it was only available for part of the study period due to equipment malfunction and loss and a combination of sweater temperature and total monthly rainfall. Therefore seawater could be used to try and predict future changes in sponge assemblages, however, it only explains approximately $8 \%$ of the variation between seasonal samples. Air temperature showed a regular seasonal pattern, however wind, rain and atmospheric pressure were more random, which is typical for the Wellington region which is dominated by shifting northerly and southerly fronts of weather (Heath, 1971).

The sponge data collected during this study did not detect a consistent seasonal variation pattern despite monitoring every three months over the two years. 2007 to early 2009 were La Nina years and from about March 2009 it changed 
into an ENSO (El Nino Southern Oscillation) with very little neutral period. Changes in sponge assemblages in Mexico have been correlated with ENSO previously (Carballo et al., 2008). ENSO and similar climatic events are known to affect marine communities by disrupting normal recruitment and reproductive patterns (Navarrete et al., 2002; Gaymer et al., 2010) If the sponge data were collected over a much longer time span, more robust time series analysis tests could be performed which may be able to detect other longer term natural variation. This suggests the need for long term, regular monitoring of sponge assemblages, especially those that are being examined for change in order to understand the scale of the temporal variation.

Unfortunately, sponges are not seen as high priority for monitoring at the moment by conservation agencies therefore it is important to try and develop methods to monitor the sponge assemblages quickly with limited expertise and yet capture as much relevant data as possible. Therefore in Chapter 5 the potential of morphological monitoring in order to pick out significant patterns of seasonal assemblage changes will be explored over a longer time period. 
Chapter 4 Temporal variability in sponge assemblages on the Wellington South Coast, New Zealand

\subsection{APPENDIX SIMPER Similarity PERCENTAGES - TWO-WAY ANALYSIS OF SPECIES CONTRIBUTIONS OF SITE CROSSED WITH SEASON (60\% CONTRIBUTION CUT OFF). CALCAREOUS SPECIES ARE SHOWN IN BOLD.}

Table 4.3 Species explaining the similarity across all sites in autumn. Average similarity between all quadrats in autumn was $63.95 \%$

\begin{tabular}{|l|r|r|r|r|r|}
\hline Species & \multicolumn{1}{l|}{ Av.Abund } & \multicolumn{1}{l|}{ Av.Sim } & \multicolumn{1}{l|}{ Sim/SD } & \multicolumn{1}{l|}{ Contrib\% } & \multicolumn{1}{l|}{ Cum.\% } \\
\hline Clathrina sp. 2 & 3.36 & 6.77 & 1.76 & 10.58 & 10.58 \\
\hline Halisarca sp. & 3.1 & 6.45 & 3.82 & 10.09 & 20.68 \\
\hline Oscarella lobularis & 2.68 & 5.04 & 2.11 & 7.88 & 28.55 \\
\hline Stellata sp. & 2.31 & 4.98 & 3.09 & 7.78 & 36.33 \\
\hline Clathrina sp. & 2.33 & 4.06 & 1.54 & 6.35 & 42.68 \\
\hline Leucosolenia echinata & 2.12 & 3.79 & 1.59 & 5.93 & 48.61 \\
\hline Clathrina sp. 3 & 2.17 & 3.37 & 1.32 & 5.27 & 53.88 \\
\hline Clathrina sp. 4 & 1.94 & 3.28 & 0.78 & 5.12 & 59 \\
\hline Crella sp. & 1.74 & 3.06 & 1.15 & 4.79 & 63.79 \\
\hline
\end{tabular}

Table 4.4 Species explaining the similarity across all sites in spring. Average similarity between all quadrats in spring was $64.23 \%$

\begin{tabular}{|l|r|r|r|r|r|}
\hline Species & \multicolumn{1}{l|}{ Av.Abund } & \multicolumn{1}{l|}{ Av.Sim } & \multicolumn{1}{l|}{ Sim/SD } & \multicolumn{1}{l|}{ Contrib\% } & \multicolumn{1}{l|}{ Cum.\% } \\
\hline Clathrina sp. 2 & 2.54 & 6.19 & 2.18 & 9.63 & 9.63 \\
\hline Clathrina sp. 3 & 2.54 & 5.59 & 1.37 & 8.7 & 18.33 \\
\hline Stellata sp. & 1.91 & 5.39 & 2.92 & 8.39 & 26.71 \\
\hline Leucosolenia echinata & 1.88 & 5.05 & 1.36 & 7.86 & 34.58 \\
\hline Crella sp. & 1.83 & 5 & 2.25 & 7.79 & 42.37 \\
\hline Sycon sp. & 1.83 & 4.81 & 1.17 & 7.48 & 49.85 \\
\hline Halisarca sp. & 2.16 & 4.48 & 1.25 & 6.98 & 56.83 \\
\hline Clathrina sp. & 1.91 & 3.66 & 1.39 & 5.7 & 62.53 \\
\hline
\end{tabular}

Table 4.5 Species explaining the similarity across all sites in summer. Average similarity between all quadrats in summer was $66.49 \%$

\begin{tabular}{|l|r|r|r|r|r|}
\hline Species & \multicolumn{1}{|l}{ Av.Abund } & \multicolumn{1}{l|}{ Av.Sim } & \multicolumn{1}{l|}{ Sim/SD } & \multicolumn{1}{l|}{ Contrib\% } & \multicolumn{1}{l|}{ lum.\% } \\
\hline Clathrina sp. 2 & 3.24 & 6.81 & 1.82 & 10.24 & 10.24 \\
\hline Leucosolenia echinata & 2.57 & 6.39 & 3.06 & 9.61 & 19.85 \\
\hline Sycon sp. & 2.53 & 6.24 & 1.52 & 9.39 & 29.23 \\
\hline Halisarca sp. & 2.55 & 5.42 & 2.27 & 8.15 & 37.38 \\
\hline Clathrina sp. 3 & 2.7 & 5.21 & 1.29 & 7.83 & 45.22 \\
\hline Oscarella lobularis & 2.27 & 4.9 & 1.35 & 7.37 & 52.58 \\
\hline Crella sp. & 2.02 & 4.82 & 1.36 & 7.25 & 59.84 \\
\hline Clathrina sp. 4 & 2.55 & 4.67 & 1.89 & 7.02 & 66.86 \\
\hline
\end{tabular}


Chapter 4 Temporal variability in sponge assemblages on the Wellington South Coast, New Zealand

Table 4.6 Species explaining the similarity across all sites in winter. Average similarity between all quadrats in winter was $67.59 \%$

\begin{tabular}{|l|r|r|r|r|r|}
\hline Species & \multicolumn{1}{|l|}{ Av.Abund } & \multicolumn{1}{l|}{ Av.Sim } & \multicolumn{1}{l|}{ Sim/SD } & \multicolumn{1}{l|}{ Contrib\% } & \multicolumn{1}{l|}{ Cum.\% } \\
\hline Clathrina sp. 2 & 3.01 & 10.96 & 1.47 & 16.22 & 16.22 \\
\hline Stellata sp. & 1.95 & 7.83 & 3.59 & 11.59 & 27.8 \\
\hline Sycon sp. & 1.97 & 7.24 & 1.27 & 10.7 & 38.51 \\
\hline Oscarella lobularis & 1.79 & 5.61 & 1.73 & 8.3 & 46.8 \\
\hline Clathrina sp. 3 & 1.65 & 5.37 & 1.28 & 7.94 & 54.75 \\
\hline Crella sp. & 1.31 & 4.36 & 1.21 & 6.46 & 61.2 \\
\hline Halisarca sp. & 1.55 & 3.99 & 1.05 & 5.9 & 67.11 \\
\hline
\end{tabular}

Table 4.7 Species explaining the differences between autumn and spring. Average percentage differences between all quadrats in autumn and spring was $42.12 \%$

\begin{tabular}{|c|c|c|c|c|c|c|}
\hline Species & $\begin{array}{l}\text { Av. } \\
\text { Abund. } \\
\text { autumn }\end{array}$ & $\begin{array}{l}\text { Av. } \\
\text { Abund } \\
\text { spring }\end{array}$ & Av. Diss & $\begin{array}{l}\text { Diss/ } \\
\text { SD }\end{array}$ & Contrib\% & Cum.\% \\
\hline Clathrina sp. 2 & 2.17 & 2.54 & 2.26 & 1.44 & 5.37 & 5.37 \\
\hline Halisarca sp. & 3.1 & 2.16 & 2.08 & 1.3 & 4.95 & 10.32 \\
\hline Clathrina sp. & 2.33 & 1.91 & 1.96 & 1.36 & 4.65 & 14.97 \\
\hline Oscarella lobularis & 2.68 & 1.92 & 1.93 & 1.26 & 4.58 & 19.55 \\
\hline Clathrina sp. 3 & 3.36 & 2.54 & 1.81 & 1.55 & 4.3 & 23.85 \\
\hline Crella sp. & 1.74 & 1.83 & 1.65 & 1.45 & 3.91 & 27.76 \\
\hline Leucosolenia echinata & 2.12 & 1.88 & 1.62 & 1.11 & 3.85 & 31.61 \\
\hline Sycon sp. & 1.17 & 1.83 & 1.59 & 1.4 & 3.78 & 35.39 \\
\hline Strongylacidon conulosa & 1.36 & 1.31 & 1.55 & 1.23 & 3.69 & 39.07 \\
\hline Leucetta sp. 1 & 1.51 & 1.41 & 1.42 & 1.34 & 3.38 & 42.45 \\
\hline Plakina c.f trilopha & 1.87 & 1.54 & 1.38 & 1.09 & 3.28 & 45.74 \\
\hline Mycale sp. & 0.93 & 0.7 & 1.35 & 0.75 & 3.22 & 48.95 \\
\hline Clathrina sp. 1 & 1.94 & 1.4 & 1.21 & 0.94 & 2.87 & 51.82 \\
\hline Haliclona venustina & 0.88 & 0.57 & 1.12 & 1.09 & 2.66 & 54.48 \\
\hline Ancorina alata & 1.41 & 1.33 & 1.12 & 1.32 & 2.66 & 57.14 \\
\hline Callyspongia sp. & 0.84 & 0.18 & 1.12 & 0.89 & 2.65 & 59.79 \\
\hline Oscarella sp. & 0.41 & 0.41 & 1.01 & 0.76 & 2.4 & 62.19 \\
\hline
\end{tabular}


Chapter 4 Temporal variability in sponge assemblages on the Wellington South Coast, New Zealand

Table 4.8 Species explaining the differences between autumn and summer. Average percentage differences between all quadrats in autumn and summer was $39.01 \%$

\begin{tabular}{|l|r|r|r|r|r|r|}
\hline & \multicolumn{1}{|c|}{$\begin{array}{l}\text { Av.Abund } \\
\text { autumn }\end{array}$} & $\begin{array}{l}\text { Av.Abund } \\
\text { summer }\end{array}$ & Av.Diss & Diss/SD & Contrib\% & \multicolumn{1}{l|}{ Cum.\% } \\
\hline Sycon sp. & 1.17 & 2.53 & 2.1 & 1.79 & 5.37 & 5.37 \\
\hline Clathrina sp. & 2.33 & 2.26 & 2.06 & 1.21 & 5.28 & 10.65 \\
\hline Clathrina sp. 3 & 2.17 & 2.55 & 1.72 & 1.19 & 4.4 & 15.06 \\
\hline Crella sp. & 1.74 & 2.02 & 1.55 & 1.18 & 3.98 & 19.03 \\
\hline Oscarella lobularis & 2.68 & 2.27 & 1.52 & 1.15 & 3.89 & 22.92 \\
\hline Clathrina sp. 1 & 1.94 & 2.7 & 1.44 & 0.81 & 3.68 & 26.61 \\
\hline Plakina c.f trilopha & 1.87 & 1.68 & 1.36 & 1 & 3.49 & 30.1 \\
\hline Leucetta sp. 1 & 1.51 & 1.25 & 1.36 & 1.18 & 3.47 & 33.57 \\
\hline Halisarca sp. & 3.1 & 2.55 & 1.35 & 1.05 & 3.46 & 37.03 \\
\hline Mycale sp. & 0.93 & 0.38 & 1.33 & 0.78 & 3.41 & 40.44 \\
\hline Strongylacidon conulosa & 1.36 & 1.5 & 1.33 & 1.15 & 3.4 & 43.84 \\
\hline Haliclona venustina & 0.88 & 1.08 & 1.31 & 1.11 & 3.35 & 47.18 \\
\hline Leucosolenia echinata & 2.12 & 2.57 & 1.25 & 0.89 & 3.19 & 50.38 \\
\hline Callyspongia sp. & 0.84 & 0.25 & 1.16 & 0.96 & 2.96 & 53.34 \\
\hline Clathrina sp. 2 & 3.36 & 3.24 & 1.14 & 1.12 & 2.93 & 56.27 \\
\hline Haliclona sp. & 0.4 & 0.56 & 1.07 & 1.08 & 2.74 & 59.02 \\
\hline Stellata sp. & 2.31 & 1.77 & 1.04 & 1.14 & 2.68 & 61.69 \\
\hline
\end{tabular}

Table 4.9 Species explaining the differences between spring and summer. Average percentage differences between all quadrats in spring and summer was $39.64 \%$.

\begin{tabular}{|l|r|r|r|r|r|r|}
\hline Species & \multicolumn{1}{|l|}{$\begin{array}{l}\text { Av.Abund } \\
\text { spring }\end{array}$} & $\begin{array}{l}\text { Av.Abund } \\
\text { summer }\end{array}$ & Av.Diss & Diss/SD & Contrib\% & \multicolumn{1}{c|}{ Cum.\% } \\
\hline Clathrina sp. & 1.91 & 2.26 & 2.38 & 1.22 & 6 & 6 \\
\hline Clathrina sp. 3 & 1.4 & 2.7 & 2.2 & 1.18 & 5.55 & 11.56 \\
\hline Oscarella lobularis & 1.92 & 2.27 & 2.08 & 1.26 & 5.24 & 16.8 \\
\hline Clathrina sp. 4 & 2.54 & 2.55 & 2.07 & 1.26 & 5.22 & 22.02 \\
\hline Clathrina sp. 2 & 2.54 & 3.24 & 1.93 & 1.47 & 4.86 & 26.88 \\
\hline Halisarca sp. & 2.16 & 2.55 & 1.84 & 1.14 & 4.63 & 31.51 \\
\hline Strongylacidon conulosa & 1.31 & 1.5 & 1.58 & 1.2 & 3.99 & 35.5 \\
\hline Plakina c.f trilopha & 1.54 & 1.68 & 1.58 & 1.32 & 3.98 & 39.47 \\
\hline Haliclona venustina & 0.57 & 1.08 & 1.57 & 1.13 & 3.95 & 43.42 \\
\hline Sycon sp. & 1.83 & 2.53 & 1.55 & 1.37 & 3.91 & 47.33 \\
\hline Ancorina alata & 1.33 & 1.04 & 1.33 & 1.26 & 3.35 & 50.68 \\
\hline Mycale sp. & 0.7 & 0.38 & 1.32 & 0.76 & 3.34 & 54.02 \\
\hline Leucosolenia echinata & 1.88 & 2.57 & 1.32 & 1.32 & 3.34 & 57.36 \\
\hline Crella sp. & 1.83 & 2.02 & 1.28 & 1.21 & 3.23 & 60.59 \\
\hline
\end{tabular}


Chapter 4 Temporal variability in sponge assemblages on the Wellington South Coast, New Zealand

Table 4.10 Species explaining the differences between autumn and winter. Average percentage differences between all quadrats in autumn and winter was $51.98 \%$

\begin{tabular}{|l|r|r|r|r|r|r|}
\hline & $\begin{array}{l}\text { Av.Abund } \\
\text { autumn }\end{array}$ & \multicolumn{1}{|c|}{$\begin{array}{l}\text { Av.Abund } \\
\text { winter }\end{array}$} & Av.Diss & Diss/SD & Contrib\% & \multicolumn{1}{l|}{ Cum.\% } \\
\hline Halisarca sp. & 3.1 & 1.55 & 3.19 & 1.62 & 6.14 & 6.14 \\
\hline Clathrina sp. & 2.33 & 0.61 & 3.04 & 1.57 & 5.85 & 11.99 \\
\hline Leucosolenia echinata & 2.12 & 0.35 & 3.01 & 1.57 & 5.79 & 17.78 \\
\hline Clathrina sp. 3 & 2.17 & 1.01 & 2.91 & 1.54 & 5.59 & 23.37 \\
\hline Plakina c.f trilopha & 1.87 & 1.33 & 2.63 & 1.35 & 5.05 & 28.42 \\
\hline Clathrina sp. 4 & 1.94 & 1.65 & 2.62 & 1.22 & 5.03 & 33.46 \\
\hline Oscarella lobularis & 2.68 & 1.79 & 2.35 & 1.68 & 4.52 & 37.98 \\
\hline Sycon sp. & 1.17 & 1.97 & 2.07 & 1.22 & 3.99 & 41.97 \\
\hline Leucetta sp. 1 & 1.51 & 0.52 & 1.8 & 1.31 & 3.46 & 45.43 \\
\hline Strongylacidon conulosa & 1.36 & 0.62 & 1.77 & 1.24 & 3.41 & 48.84 \\
\hline Clathrina sp. 2 & 3.36 & 3.01 & 1.69 & 1.51 & 3.26 & 52.1 \\
\hline Crella sp. & 1.74 & 1.31 & 1.67 & 1.32 & 3.22 & 55.32 \\
\hline Ancorina alata & 1.41 & 1.11 & 1.41 & 1.44 & 2.71 & 58.03 \\
\hline Callyspongia sp. & 0.84 & 0.12 & 1.37 & 1.05 & 2.64 & 60.67 \\
\hline
\end{tabular}

Table 4.11 Species explaining the differences between spring and winter. Average percentage differences between all quadrats in spring and winter was $48.02 \%$

\begin{tabular}{|l|r|r|r|r|r|r|}
\hline & \multicolumn{1}{|c|}{$\begin{array}{l}\text { Av.Abund } \\
\text { spring }\end{array}$} & \multicolumn{1}{|c|}{$\begin{array}{l}\text { Av.Abund } \\
\text { winter }\end{array}$} & Av.Diss & Diss/SD & Contrib\% & \multicolumn{1}{c|}{ Cum.\% } \\
\hline Leucosolenia echinata & 1.88 & 0.35 & 3.61 & 1.48 & 7.51 & 7.51 \\
\hline Clathrina sp. 3 & 2.54 & 1.01 & 3.27 & 1.35 & 6.8 & 14.32 \\
\hline Clathrina sp. & 1.91 & 0.61 & 3.09 & 1.33 & 6.44 & 20.75 \\
\hline Sycon sp. & 1.83 & 1.97 & 2.67 & 1.55 & 5.55 & 26.31 \\
\hline Oscarella lobularis & 1.92 & 1.79 & 2.48 & 1.46 & 5.17 & 31.47 \\
\hline Plakina c.f trilopha & 1.54 & 1.33 & 2.4 & 1.36 & 4.99 & 36.46 \\
\hline Clathrina sp. 4 & 1.4 & 1.65 & 2.39 & 1.23 & 4.98 & 41.45 \\
\hline Crella sp. & 1.83 & 1.31 & 2.29 & 1.28 & 4.77 & 46.22 \\
\hline Halisarca sp. & 2.16 & 1.55 & 2.16 & 1.19 & 4.5 & 50.72 \\
\hline Leucetta sp. 1 & 1.41 & 0.52 & 1.98 & 1.09 & 4.12 & 54.84 \\
\hline Clathrina sp. 2 & 2.54 & 3.01 & 1.89 & 1.11 & 3.94 & 58.78 \\
\hline Strongylacidon conulosa & 1.31 & 0.62 & 1.66 & 1.21 & 3.45 & 62.23 \\
\hline
\end{tabular}


Chapter 4 Temporal variability in sponge assemblages on the Wellington South Coast, New Zealand

Table 4.12 Species explaining the differences between summer and winter. Average percentage differences between all quadrats in summer and winter was $48.73 \%$.

\begin{tabular}{|l|r|r|r|r|r|r|}
\hline & $\begin{array}{l}\text { Av.Abund } \\
\text { summer }\end{array}$ & $\begin{array}{l}\text { Av.Abund } \\
\text { winter }\end{array}$ & Av.Diss & Diss/SD & Contrib\% & \multicolumn{1}{c|}{ Cum.\% } \\
\hline Leucosolenia echinata & 2.57 & 0.35 & 3.84 & 1.92 & 7.88 & 7.88 \\
\hline Clathrina sp. & 2.26 & 0.61 & 3.43 & 1.22 & 7.05 & 14.93 \\
\hline Clathrina sp. 4 & 2.55 & 1.01 & 3.32 & 1.59 & 6.81 & 21.74 \\
\hline Plakina c.f trilopha & 1.68 & 1.33 & 3.07 & 1.62 & 6.3 & 28.04 \\
\hline Halisarca sp. & 2.55 & 1.55 & 2.81 & 1.54 & 5.76 & 33.81 \\
\hline Sycon sp. & 2.53 & 1.97 & 2.34 & 1.44 & 4.79 & 38.6 \\
\hline Clathrina sp. 1 & 2.7 & 1.65 & 2.17 & 1.06 & 4.46 & 43.06 \\
\hline Crella sp. & 2.02 & 1.31 & 2.12 & 1.54 & 4.35 & 47.41 \\
\hline Clathrina sp. 2 & 3.24 & 3.01 & 2.03 & 1.39 & 4.17 & 51.58 \\
\hline Oscarella lobularis & 2.27 & 1.79 & 1.88 & 1.18 & 3.85 & 55.44 \\
\hline Haliclona venustina & 1.08 & 0.81 & 1.87 & 1.14 & 3.83 & 59.27 \\
\hline Strongylacidon conulosa & 1.5 & 0.62 & 1.86 & 1.29 & 3.82 & 63.09 \\
\hline
\end{tabular}

Table 4.13 Species explaining the similarities within the Breaker Bay quadrats. The percentage similarity across all the seasons was $57.70 \%$.

\begin{tabular}{|l|r|r|r|r|r|}
\hline Species & \multicolumn{1}{|l}{ Av.Abund } & \multicolumn{1}{l|}{ Av.Sim } & \multicolumn{1}{l|}{ Sim/SD } & \multicolumn{1}{l|}{ Contrib\% } & \multicolumn{1}{l|}{ Cum.\% } \\
\hline Sycon sp. & 2.49 & 8.14 & 2 & 14.11 & 14.11 \\
\hline Stellata sp. & 2.02 & 6.64 & 3.23 & 11.51 & 25.62 \\
\hline Crella sp. & 2.06 & 6.19 & 2.2 & 10.72 & 36.34 \\
\hline Leucosolenia echinata & 1.74 & 5.32 & 1.32 & 9.22 & 45.56 \\
\hline Clathrina sp. 4 & 1.7 & 4.35 & 1.27 & 7.55 & 53.11 \\
\hline Ancorina alata & 1.28 & 3.83 & 1.65 & 6.64 & 59.75 \\
\hline Mycale sp. & 1.54 & 3.57 & 0.98 & 6.18 & 65.93 \\
\hline
\end{tabular}

Table 4.14 Species explaining the similarities within the Far Sirens quadrats. The percentage similarity across all the seasons was $64.03 \%$.

\begin{tabular}{|l|r|r|r|r|r|}
\hline Species & Av.Abund & \multicolumn{1}{l|}{ Av.Sim } & \multicolumn{1}{l|}{ Sim/SD } & \multicolumn{1}{l|}{ Contrib\% } & \multicolumn{1}{l|}{ Cum.\% } \\
\hline Clathrina sp. 2 & 3.77 & 9.72 & 3.42 & 15.18 & 15.18 \\
\hline Halisarca sp. & 3.04 & 7.55 & 3.44 & 11.79 & 26.97 \\
\hline Oscarella lobularis & 2.63 & 5.78 & 2.47 & 9.03 & 36 \\
\hline Stellata sp. & 2.21 & 5.63 & 3.9 & 8.8 & 44.8 \\
\hline Leucetta sp. 1 & 1.79 & 4.08 & 1.67 & 6.38 & 51.17 \\
\hline Plakina c.f trilopha & 2.08 & 4.08 & 1.19 & 6.37 & 57.55 \\
\hline Clathrina sp. 4 & 1.85 & 4.01 & 1.18 & 6.26 & 63.81 \\
\hline
\end{tabular}


Chapter 4 Temporal variability in sponge assemblages on the Wellington South Coast, New Zealand

Table 4.15 Species explaining the similarities within the Near Sirens quadrats. The percentage similarity across all the seasons was $72.46 \%$.

\begin{tabular}{|l|r|r|r|r|r|}
\hline Species & Av.Abund & \multicolumn{1}{l|}{ Av.Sim } & \multicolumn{1}{l|}{ Sim/SD } & \multicolumn{1}{l|}{ Contrib\% } & \multicolumn{1}{l|}{ Cum.\% } \\
\hline Clathrina sp. 2 & 4.28 & 9.41 & 2.43 & 12.98 & 12.98 \\
\hline Clathrina sp. 3 & 3.83 & 8.11 & 4.88 & 11.19 & 24.17 \\
\hline Halisarca sp. & 2.77 & 5.2 & 2.58 & 7.18 & 31.35 \\
\hline Clathrina sp. 4 & 2.86 & 5.07 & 2.48 & 7 & 38.35 \\
\hline Leucosolenia echinata & 2.45 & 5.03 & 2.37 & 6.94 & 45.29 \\
\hline Oscarella lobularis & 2.72 & 4.91 & 1.92 & 6.78 & 52.07 \\
\hline Clathrina sp. & 2.74 & 4.66 & 1.81 & 6.44 & 58.51 \\
\hline Plakina c.f trilopha & 2.37 & 4.64 & 2.43 & 6.41 & 64.91 \\
\hline
\end{tabular}

Table 4.16 Species explaining the differences between Breaker Bay and the Far Sirens quadrats across all seasons. Average percentage difference between the Breaker Bay and Far Sirens quadrats $55.98 \%$.

\begin{tabular}{|c|c|c|c|c|c|c|}
\hline Species & $\begin{array}{l}\text { Av.Abund } \\
\text { Breaker } \\
\text { Bay }\end{array}$ & $\begin{array}{l}\text { Av.Abund } \\
\text { Far Sirens }\end{array}$ & Av.Diss & Diss/SD & Contrib\% & Cum.\% \\
\hline Clathrina sp. 2 & 0.98 & 3.77 & 4.78 & 1.94 & 8.54 & 8.54 \\
\hline Halisarca sp. & 1.45 & 3.04 & 3.18 & 1.41 & 5.68 & 14.21 \\
\hline Plakina c.f trilopha & 0.45 & 2.08 & 3.04 & 1.47 & 5.44 & 19.65 \\
\hline Oscarella lobularis & 1.25 & 2.63 & 2.68 & 1.49 & 4.79 & 24.44 \\
\hline Mycale sp. & 1.54 & 0.22 & 2.54 & 1.38 & 4.53 & 28.97 \\
\hline Sycon sp. & 2.49 & 1.45 & 2.52 & 1.62 & 4.5 & 33.47 \\
\hline Leucetta sp. 1 & 0.51 & 1.79 & 2.43 & 1.72 & 4.34 & 37.82 \\
\hline Leucosolenia echinata & 1.74 & 1.16 & 2.18 & 1.29 & 3.9 & 41.71 \\
\hline Clathrina sp. & 1.34 & 1.48 & 2.14 & 1.23 & 3.83 & 45.54 \\
\hline Clathrina sp. 4 & 1.7 & 1.85 & 2.14 & 1.31 & 3.82 & 49.36 \\
\hline Clathrina sp. 3 & 0.22 & 1.46 & 2.03 & 0.86 & 3.62 & 52.98 \\
\hline Haliclona venustina & 0.44 & 1.45 & 1.98 & 1.31 & 3.54 & 56.52 \\
\hline Strongylacidon conulosa & 0.63 & 0.85 & 1.73 & 1.19 & 3.09 & 59.6 \\
\hline Crella sp. & 2.06 & 1.66 & 1.69 & 1.42 & 3.02 & 62.62 \\
\hline
\end{tabular}


Chapter 4 Temporal variability in sponge assemblages on the Wellington South Coast, New Zealand

Table 4.17 Species explaining the differences between Breaker Bay and the Near Sirens quadrats across all seasons. Average percentage difference between the Breaker Bay and Near Sirens quadrats $56.09 \%$.

\begin{tabular}{|c|c|c|c|c|c|c|}
\hline Species & $\begin{array}{l}\text { Av.Abund } \\
\text { Breaker } \\
\text { Bay }\end{array}$ & $\begin{array}{l}\text { Av.Abund } \\
\text { Near } \\
\text { Sirens }\end{array}$ & Av.Diss & Diss/SD & Contrib\% & Cum.\% \\
\hline Clathrina sp. 3 & 0.22 & 3.83 & 5.2 & 3.66 & 9.27 & 9.27 \\
\hline Clathrina sp. 2 & 0.98 & 4.28 & 4.89 & 1.87 & 8.72 & 17.99 \\
\hline Plakina c.f trilopha & 0.45 & 2.37 & 2.93 & 2.11 & 5.23 & 23.22 \\
\hline Dysidea sp. 1 & 0.35 & 2.29 & 2.79 & 2.13 & 4.97 & 28.19 \\
\hline Clathrina sp. & 1.34 & 2.74 & 2.6 & 1.58 & 4.64 & 32.82 \\
\hline Oscarella lobularis & 1.25 & 2.72 & 2.55 & 1.67 & 4.55 & 37.38 \\
\hline Sycon sp. & 2.49 & 1.34 & 2.48 & 1.05 & 4.43 & 41.8 \\
\hline Strongylacidon conulosa & 0.63 & 2.18 & 2.47 & 1.82 & 4.4 & 46.2 \\
\hline Halisarca sp. & 1.45 & 2.77 & 2.35 & 1.54 & 4.19 & 50.39 \\
\hline Clathrina sp. 4 & 1.7 & 2.86 & 2.29 & 1.48 & 4.07 & 54.47 \\
\hline Mycale sp. & 1.54 & 0.2 & 2.28 & 1.3 & 4.06 & 58.53 \\
\hline Leucetta sp. 1 & 0.51 & 1.46 & 1.87 & 1.26 & 3.34 & 61.87 \\
\hline
\end{tabular}

Table 4.18 Species explaining the differences between Far Sirens and the Near Sirens quadrats across all seasons. Average percentage difference between the Far Sirens and Near Sirens quadrats $46.10 \%$.

\begin{tabular}{|l|r|r|r|r|r|r|}
\hline & $\begin{array}{l}\text { Av.Abund } \\
\text { Far Sirens }\end{array}$ & \multicolumn{1}{l|}{$\begin{array}{l}\text { Near } \\
\text { Sirens }\end{array}$} & Av.Diss & Diss/SD & Contrib\% & \multicolumn{1}{l|}{ Cum.\% } \\
\hline Clathrina sp. 3 & 1.46 & 3.83 & 3.83 & 2.2 & 8.3 & 8.3 \\
\hline Dysidea sp. 1 & 0.35 & 2.29 & 2.64 & 1.99 & 5.72 & 14.02 \\
\hline Plakina c.f trilopha & 2.08 & 2.37 & 2.3 & 1.17 & 5 & 19.01 \\
\hline Clathrina sp. 4 & 1.85 & 2.86 & 2.29 & 1.48 & 4.96 & 23.98 \\
\hline Clathrina sp. & 1.48 & 2.74 & 2.22 & 1.46 & 4.81 & 28.79 \\
\hline Sycon sp. & 1.45 & 1.34 & 2.15 & 1.1 & 4.66 & 33.44 \\
\hline Leucosolenia echinata & 1.16 & 2.45 & 2.11 & 1.56 & 4.57 & 38.02 \\
\hline Strongylacidon conulosa & 0.85 & 2.18 & 2.1 & 1.67 & 4.56 & 42.58 \\
\hline Crella sp. & 1.66 & 1.48 & 1.93 & 1.44 & 4.19 & 46.77 \\
\hline Oscarella lobularis & 2.63 & 2.72 & 1.79 & 1.45 & 3.89 & 50.65 \\
\hline Halisarca sp. & 3.04 & 2.77 & 1.76 & 1.08 & 3.83 & 54.48 \\
\hline Haliclona venustina & 1.45 & 0.53 & 1.67 & 1.31 & 3.61 & 58.09 \\
\hline Leucetta sp. 1 & 1.79 & 1.46 & 1.54 & 1.3 & 3.33 & 61.42 \\
\hline
\end{tabular}


Chapter 4 Temporal variability in sponge assemblages on the Wellington South Coast, New Zealand 
CHAPTER 5

SPATIAL AND TEMPORAL VARIABILITY OF SPONGE SPECIES AND MORPHOLOGICAL DIVERSITY IN A TEMPERATE MARINE RESERVE 


\subsection{ABSTRACT}

Sponges are a dominant component of benthic assemblages in hard substratum environments across the world, which have many important functional roles. Despite the wide range of functional roles that sponges fulfill, they are generally poorly represented in most marine monitoring programmes because of taxonomic difficulties and problems in quantification. Given these problems there is considerable need to develop effective sponge monitoring tools and determine the most time, cost and expertise-efficient way to monitor changes in sponge assemblages. Skomer Marine Reserve (MNR), Wales, UK has been previously identified as having rich and diverse sponge assemblages and was the focus of previous research examining temporal changes in sponge assemblages. Morphological monitoring is one surrogate method that could be a useful addition to any environmental monitoring toolkit. This chapter investigates whether or not species level and morphological level data sets were highly correlated with respect to both spatial and temporal variation. Both data sets revealed significant seasonal shifts in assemblage structure and significant differences between sites. However, annual changes were not significant in either case. The physical factors correlating with changes in assemblage structure were mean monthly water temperature, maximum monthly air temperature, net monthly radiation and monthly maximum wind gust speed. All of these environmental factors were highly correlated with seasonal variability. It is recommended that an initial intensive species survey is carried out prior to using a morphological approach to ensure the two variables correlate. 


\subsection{INTRODUCTION}

Sponges are a dominant component of worldwide benthic environments (Dayton, 1978; Alvarez et al., 1990; Diaz et al., 1993; Økland \& Økland, 1996; Bell \& Barnes, 2000c; Hooper et al., 2002; Bell \& Smith, 2004; Leys et al., 2004). Despite the wide range of functional roles that sponges fulfill they are still poorly represented in most marine monitoring programmes. This problem is due to time limits imposed on underwater research and sponges are thought of as a difficult group to work with because: they are taxonomically difficult and are hard to identify easily in the field (Rützler, 1978; Wulff, 2001); they show considerable phenotypic variability in response to different environmental regimes (Bell \& Barnes, 2001, 2002); and because they are difficult to quantify. It is important therefore to determine what are the most time, cost and expertise-efficient ways to monitor changes in sponge assemblages, such that they can easily and effectively be included in existing and future monitoring programmes.

Due to the difficulty in reliably identifying sponges, morphological monitoring methods (using the shape rather than species name to group sponges) have been proposed as a surrogate in order to represent the functional diversity present and get an approximation for the patterns of biodiversity (Bell \& Barnes, 2001, 2002; Bell et al., 2006; Bell, 2007a, b). Functional diversity is an important component of biodiversity for understanding the ecosystem processes and the extent of functional differences within a community (Petchey \& Gaston, 2002). It is often measured by counting the number of functional groups represented by the species within the community (Tilman et al., 2001).

Surrogates can be a time/cost effective method to represent the patterns of biodiversity within an area instead of collecting species level data. Physical surrogates include using environmental data such as sea temperature, sedimentation composition and salinity and habitat information, such as depth and latitude (McArthur et al., 2010). Biological surrogates normally use wellknown indicator groups or use lower taxonomic resolution as in chapter 2 for patterns of New Zealand sponge biodiversity. 
The majority of studies examining variability in sponge assemblages have examined a single geographic location at a single sampling interval (Barnes \& Bell, 2002; Cleary et al., 2005; Janussen \& Tendal, 2007; Picton \& Goodwin, 2007; Duckworth et al., 2008; Berman \& Bell, 2010) or a specific genus (Duckworth \& Battershill, 2001; Klautau \& Valentine, 2003), family (Plotkin, 2004) or functional role (Lemloh et al., 2009) at the regional spatial scale. Temporal variation has mostly been explored at the species level (Garrabou \& Zabala, 2001; de Caralt et al., 2008; Koopmans \& Wijffels, 2008; McMurray et al., 2008) and to date, there have been relatively few studies examining temporal variation in entire sponge assemblages over more than a year (but see chapters 4 and 6 in this thesis; Sara, 1970; Ayling, 1983b, a; Pansini \& Pronzato, 1985; Bell et al., 2006; Carballo et al., 2008). Research from the Mediterranean has shown slow growth within sponge assemblages with no clear seasonality and only some sponge tissue regression, while the abundance of different sponge species remained similar throughout an eight year study (Sara, 1970; Pansini \& Pronzato, 1985). Similar results showing little overall change have also been reported from sites in the Atlantic (Fowler \& Laffoley, 1993; Hiscock, 1994) and sites in the Caribbean (Hughes, 1996). In fact, even though the overall abundance of sponge individuals and percentage cover within assemblages remained relatively constant in the Caribbean sites, the actual individual sponges were very variable suggesting recruitment and mortality were in equilibrium in these assemblages (Hughes, 1996). However, more recent work in the Atlantic, Caribbean and Pacific suggests that more rapid changes in sponge assemblages are common and recruitment and mortality do not always occur in equilibrium and therefore the overall assemblage composition can show dramatic changes happen at the same rate (Bell et al., 2006; Wulff, 2006a; Carballo et al., 2008).

Assemblage changes can be driven by processes occurring over short temporal scales, such as wind speed and sediment deposition or across large-scale structuring factors such as the Southern Oscillation (Carballo et al., 2008).

The sponge assemblages of Skomer Marine National Reserve (MNR), Pembrokeshire, Wales, UK, have been monitored morphologically over a ten 
year period prior to this study and although there were changes between some years in morphologies present and their abundance, there was evidence the sponge assemblages could recover rapidly within a year to their original assemblage composition (Bell et al., 2006). In this previous work, no correlation was found between environmental variables and changes in species assemblage, therefore changes were attributed to natural variation or shortterm impacts (Bell et al., 2006). The limitations of this study were the lack of independence and replication of the transects and quadrats (which were predefined by Countryside Council for Wales - CCW) therefore it was not possible to draw statistically supported conclusions about changes in sponge abundance or specific morphological types over time. Also the monitoring was annual therefore it is unknown if there were seasonal changes within the assemblages. Finally, this previous research was based solely on morphological data to detect temporal changes and it was not possible to test if morphological data correlated with species data.

The aims of this study were to:

1. Quantify the patterns of seasonal variability in the sponge diversity and abundance within Skomer MNR?

2. Measure seasonal variability in the sponge morphological assemblages in Skomer MNR?

3. Determine if the patterns of species and morphological assemblage variability correlate?

4. Identify the environmental factors that best explain patterns of Skomer MNR sponge assemblage species and morphological variability? 


\subsection{METHODS}

\subsubsection{STUDY AREA}

Skomer Marine Reserve (MNR), Wales, UK has been identified as having rich and diverse sponge assemblages with over 80 sponge species being recorded to date, including six nationally scarce species and eight species that are near to the limit of their distribution (Burton et al., 2008). The MNR consists of 1500ha located off the Pembrokeshire coast of Wales (UK); 51.732 ${ }^{\circ} \mathrm{N} 5.276^{\circ} \mathrm{W}$ (Fig. 5.1). It is one of only three statutory MNRs in the UK (although more MNRs are being implemented at the moment) and was designated under the UK Wildlife and Countryside Act (1981) in 1991. There has been a comprehensive long-term monitoring programme of the intertidal and subtidal habitats of Skomer MNR since 1982 (Bullimore, 1986). Sponges have been monitored since 1993 after anecdotal evidence that there was a decline in some sponge species following dredge spoil dumping about $8 \mathrm{~km}$ away from Skomer MNR at St Annes Head (Bell et al., 2006); these observations led to dumping sites being reassigned to further offshore locations for subsequent dumping.

Photographs for this study were taken by the Countryside Council for Wales surveyed the sponge assemblages in an area called Thorn Rock off the south side of Skomer Island, which is predominately igneous bedrock covered in silt. Thorn Rock is known for having the most morphologically diverse sponge assemblages within the reserve (Lock et al., 2010a). The subtidal communities are mainly composed of bryozoan turfs, sponges and hydroids. The area is moderately exposed to south-westerly swells and the site experiences tidal currents for three hours out of the twelve hour tidal cycle, reaching speeds of 0.5-1 $\mathrm{m} \mathrm{s}^{-1}$ during spring tides. 


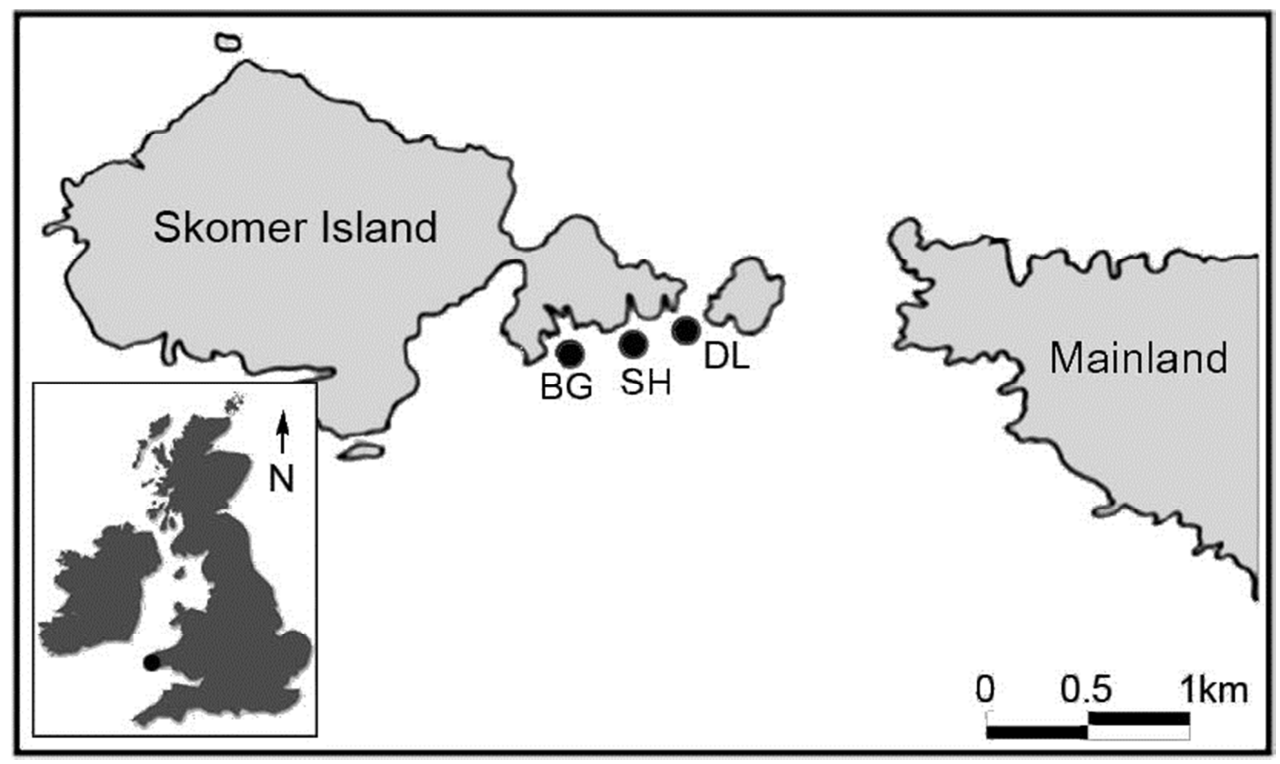

Fig. 5.1. Map of study sites within Skomer MNR. The black circle on the UK map indicates the location of Skomer MNR off the tip of the Pembrokeshire coast mainland and the site locations off Skomer Island in the Thorn Rock area which are: BG = Broad Gulley, SH = Spongy Hillocks and DL $=$ Dog Leg.

In $200515 \times 1 \mathrm{~m}^{2}$ permanent quadrats were established at three sites (five quadrats at each site) within the Thorn Rock area, less then $100 \mathrm{~m}$ apart. These sites all have horizontal bedrock and are at $18 \mathrm{~m}$ depth. An initial visual assessment of the sites suggested they had similar sponge assemblages. The three sites are called Spongy Hillocks (SH), Broad Gully (BG) and Dogleg (DL).

Each quadrat consisted of 25 cells $\left(20 \mathrm{~cm}^{2}\right)$, which were photographed using a digital camera and framer. Spongy Hillocks was first photographed in July 2006 with all three sites being photographed seasonally from spring until autumn if the weather and staffing constraints allowed (see Table 5.1 for sampling dates). Prior to taking the pictures, silt was 'wafted' so that the encrusting sponges could be seen as they are normally covered by a thin layer of silt. 
Table 5.1 Permanent quadrat surveys carried out at Thorn Rock since July 2006. The first two letters in the key represent the Site (SH: Spongy Hillocks, BG: Broad Gulley, and DL: Dog leg).

\begin{tabular}{|c|c|c|c|}
\hline Sites surveyed & Year & Month & Season \\
\hline SH & 2006 & July (7) & Summer \\
\hline SH, BG, DL & 2006 & October (10) & Autumn \\
\hline SH, BG, DL & 2007 & May (5) & Spring \\
\hline SH, BG, DL & 2007 & July (7) & Spummer \\
\hline SH, BG, DL & 2008 & May (5) & Summer \\
\hline SH, BG, DL & 2008 & July (7) & Autumn \\
\hline SH, BG, DL & 2008 & September (9) & Spring \\
\hline SH & 2009 & April (4) & Summer \\
\hline SH,BG,DL & 2009 & August (8) & \\
\hline
\end{tabular}

\subsubsection{DATA ANALYSIS}

The number of each species was counted in each cell along with the number of each morphological type simultaneously. This was important because some species, such as Cliona celata and Haliclona (Haliclona) oculata, can exhibit a range of morphologies. The species identifications have been ground-truthed on several occasions by taking small samples from sponges within the quadrat for spicule analysis during the sponge surveys of 2007 (Burton et al. 2008). Where it was difficult to distinguish species visually, particularly in the case of small patches $\left(<1 \mathrm{~cm}^{2}\right)$ and also young arborescent sponges from the generas Stelligera and Raspailia, which are similar in colour and have similar surface and gross morphology, the data collected were aggregated. The morphologies were divided into the following categories: 
Arborescent; Burrowing; Encrusting; Flabellate; Globular; Massive; Papillate; Pedunculate; Repent; and Tubular; following (Bell \& Barnes, 2001) (See Fig. $5.2)$.

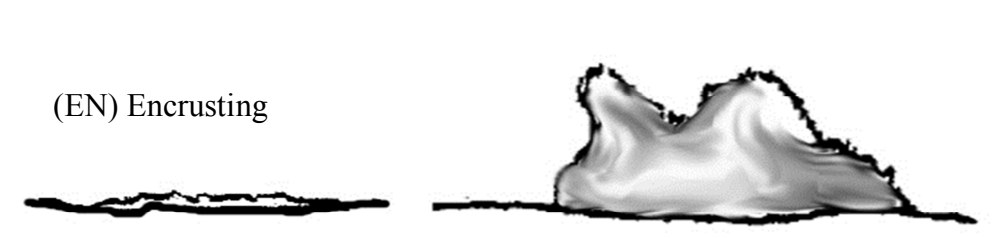

(MA) Massive

(PE) Pedunculate

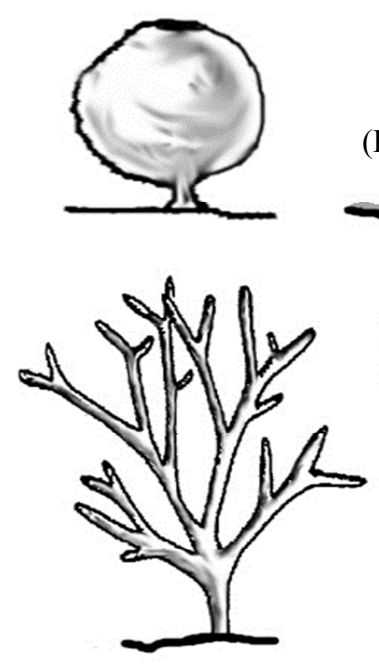

(AR) Arborescent
(BU) Burrowing

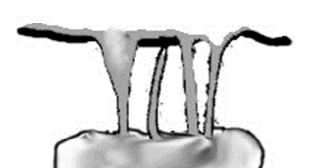

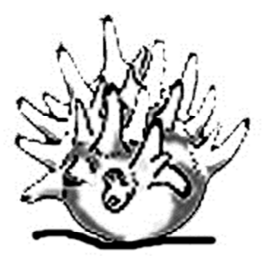

(PA) Papillate

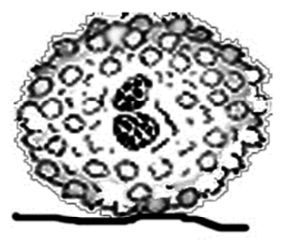

(GL) Globular

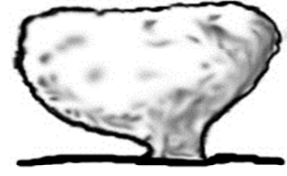

(FL) Flabellate

Figure 5.2. Morphological groups used to classify the sponges of Skomer MNR.

\subsubsection{STATISTICAL ANALYSIS}

The species and morphological richness and abundance were calculated as the mean number of species/number of sponges/morphologies found per $\mathrm{m}^{2}+$ / SE across each of the three different sites for each of the different sampling times. PRIMER (ver. 6) with the PERMANOVA add-on was used to carry out a nonparametric equivalent of a univariate ANOVA (permutational MANOVA) on the species and morphology diversity indices individually (No of species, No. of species/entities, Margalefs diversity index and Shannon Wiener Index) as response variables; this method calculates P-values using permutations, thus 
needing no assumption of a normal distribution of the data. The model used for the PERMANOVA design is shown in Table 5.2. PERMDISP was used to test for the homogeneity of beta diversity within and between the sites and within and between seasons. This was in order to understand if all three sites were actually part of one location ( a diversity) or if the three sites were different from each other and therefore representative of regional diversity ( $\beta$ diversity).

Table 5.2 PERMANOVA model design for the analysis of the multivariate species and morphological data in response to the factors listed.

\begin{tabular}{|l|c|c|c|}
\hline Factor name & Abbreviation & Type & Levels \\
\hline Site & Si & Random & 3 \\
\hline Quadrat & Qu & Fixed & 15 \\
\hline Year & Ye & Fixed & 4 \\
\hline Season & Se & Random & 3 \\
\hline
\end{tabular}

PRIMER was used to carry out ordination by non-metric Multi-Dimensional Scaling (MDS) on a dissimilarity matrix created from a Bray-Curtis similarity analysis to compare species and morphological assemblages between all three sites, between years and seasons changes to look for temporal and spatial patterns. The species data were $\log (\mathrm{x}+1)$ transformed to reduce the influence of rare species and the morphological data were square root transformed because there were only ten variables (morphological forms). MDS plots were created for the complete data set, and also for sub groups; site and season. The data from each quadrat within each site were also averaged using season nested in year as the factor and then plotted in order to compare patterns in the seasonal changes between site specific species and morphological assemblage data.

SIMPER analysis was used to elucidate the relative contribution of each species/morphological group to the average Bay-Curtis similarity between the three sites ( $\mathrm{SH}, \mathrm{DL}$, and $\mathrm{BG}$ ) and also the dissimilarity between the three seasons (spring, summer and autumn). This method determines which species were most responsible for any differences between the groups. 
PERMANOVA was used to distinguish which factors or interacting factors were significant in determining the spatial and temporal differences between the sponge assemblages. Interactions between factors are when there is an additive or multiplicative effect when there are changes in two or more factors. The change in sponge assemblage variability at a site depends on the change in seasonal variability in general in the Skomer MNR and an additive or multiplicative effect of local seasonal variability at the site. Movements in seasonal variability at the site and seasonal variability in Skomer MNR were related and/or they were interacting in a relationship to site effects on the assemblage variability.

The model used for the PERMANOVA design is the same as for the univariate assemblage structure in Table 5.2. The test statistic for PERMANOVA is the pseudo F-ratio, where a large pseudo F-ratio indicates that a factor such as location or season groups the quadrats closer in multivariate space in comparison to a random distribution and therefore identifies differences in assemblage composition. The significance of the pseudo F-ratio is tested using a permutation test that randomly shuffles the samples within and among groups and calculates the pseudo F-ratio for 9999 arbitrary reassignments of the Bray Curtis resemblance matrices for species diversity/morphological assemblages. The pseudo F-ratios of these randomly assigned communities were then compared to the pseudo F-ratio of the observed community to calculate the significance level of the test (Anderson, 2001).

The RELATE function was used to measure how closely correlated the Bray Curtis similarity matrices for the morphological and the species assemblage data were using Spearmans $\rho$. 


\subsubsection{ENVIRONMENTAL DATA}

Environmental data were collected from the Thorn Rock area by the Countryside Council for Wales within the Skomer MNR from 2006-2009 including seawater temperature, sechi disc readings, monthly air temp (mean, max and min), air pressure, sunshine hours, net radiation and maximum wind gust speed and direction. The seawater data were collected using a weekly drop down CTD probe during the field season from March-October weather permitting. The sechi disc readings have been carried out at Thorn Rock since 2004 weekly during the field season. A Campbell Scientific Environmental Change Network (ECN) compatible weather station was used to collect the weather measurements every ten minutes and has been installed and monitored by the team at CCW since April 2006. The full environmental data set has been published in the CCW Skomer marine nature reserve project status reports (Burton et al., 2007; Burton et al., 2008; Lock et al., 2009; Lock et al., 2010a).

The BEST function in PRIMER was used to determine any correlations between individual or combinations of environmental variables and the changes in the species and morphological assemblages. This uses the Spearmans rank coefficient and the BIOENV procedure to find the highest correlation for a set of environmental variables (in this case up to 5 of the variables) and the significance of the correlation was estimated using 9999 permutations. 


\subsection{RESULTS}

\subsubsection{SPECIES ASSEMBLAgE DATA.}

Fifty-nine sponge species/entities (entities are species that could not be identified to species level and were identified to a higher Operational Taxonomic unit (OTU)) were recorded across the three sites surveyed (Fig 5.3)(Table 5.3). There were 121 species/entities known from Skomer MNR therefore $48.8 \%$ of the known sponge biodiversity of the area were found within the $15 \mathrm{~m}^{2}$ surveyed.

Figure 5.3 Some of the common species and morphologies found within the Skomer MNR. a) Homoaxinella subdola b) Axinella dissimilis c) Amphilectus fucorum d) Polymastia boletiformis e) Polymastia penicillus f) Antho (Antho) involvens g) Cliona celata h) Stelligera stuposa i) Eurypon major j) Phorbas dives k) Clathria c.f. armata I) Suberites carnosus. Photos were taken by F. Bunker.

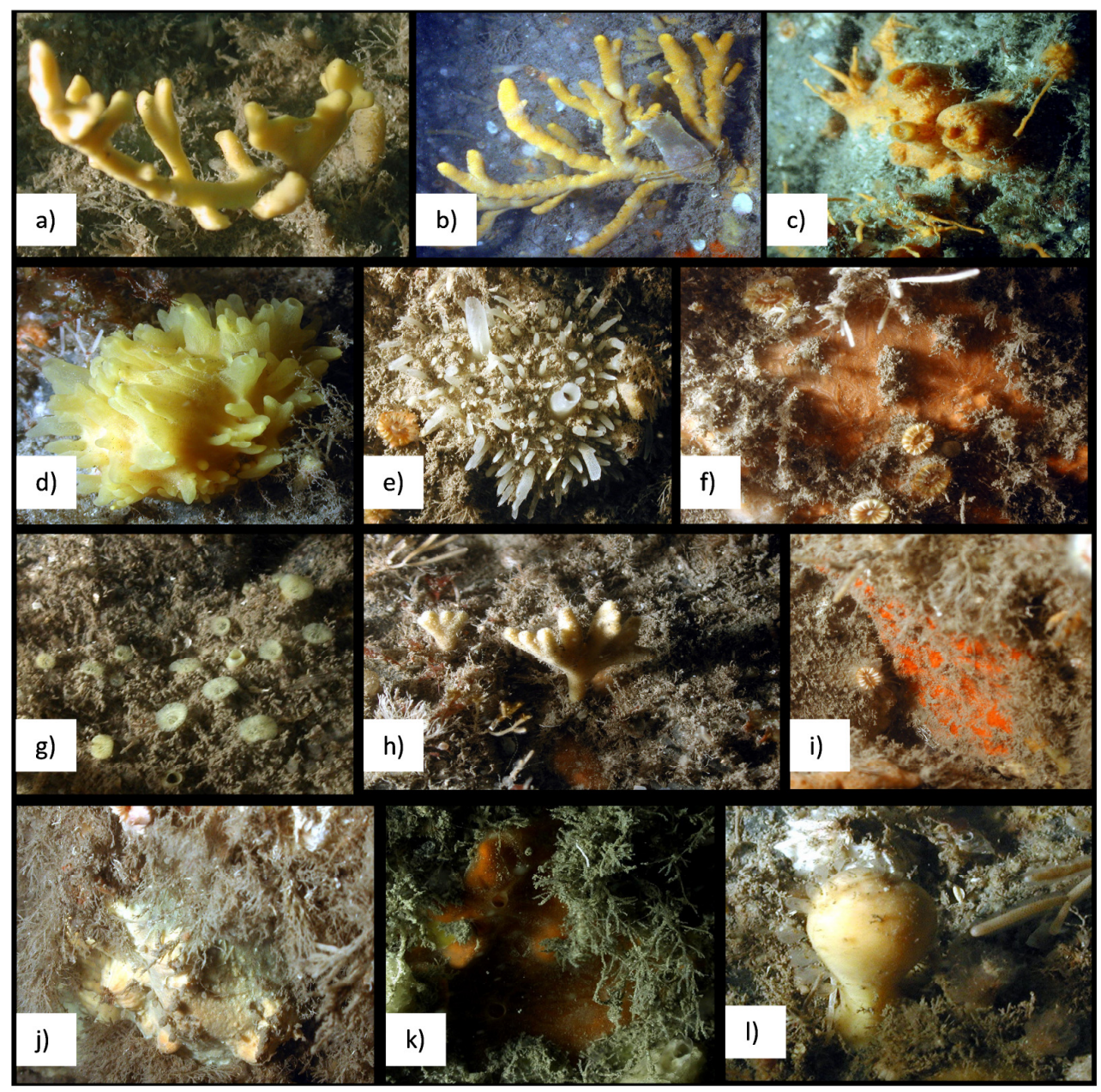


Table 5.3 Sponge species and morphologies found at the Skomer MNR. The species recorded from this present study have their morphologies listed as ar=arborescent, bu=burrowing, en=encrusting, $\mathrm{fl}=$ flabellate, $\mathrm{gl}=\mathrm{globular}, \mathrm{ma}=$ massive, $\mathrm{pa}=$ papillate, $\mathrm{pe}=$ pedunculate, $\mathrm{re}=\mathrm{repent}, \mathrm{tu}=\mathrm{tubular}$. Other studies data included were $1=$ Bell, 2006, 2 = Bunker \& Jones, 2008, 3= (ANON, 2010)

\begin{tabular}{|c|c|c|c|}
\hline Species & Morphology & $\begin{array}{l}\text { Present } \\
\text { study }\end{array}$ & $\begin{array}{l}\text { Other } \\
\text { studies }\end{array}$ \\
\hline Amphilectus fucorum (Esper, 1794) & RE & $\mathrm{Y}$ & $1,2,3$ \\
\hline Amphoriscus chrysalis (Schmidt, 1864) & & & 3 \\
\hline Antho (Acarnia) coriacea (Bowerbank, 1874) & EN & $Y$ & $1,2,3$ \\
\hline Antho (Antho) inconstans (Topsent, 1925) & EN & $\mathrm{Y}$ & 1,3 \\
\hline Antho (Antho) involvens (Schmidt, 1864) & EN & $\mathrm{Y}$ & 1,3 \\
\hline Aphroceras ensata (Bowerbank, 1858) & & & 3 \\
\hline Aplysilla sulfurea (Schulze, 1878) & & & 3 \\
\hline Axinella damicornis (Esper, 1794) & MA & $Y$ & $1,2,3$ \\
\hline Axinella dissimilis (Bowerbank, 1866) & $\mathrm{AR}$ & $\mathrm{Y}$ & $1,2,3$ \\
\hline Axinella infundibuliformis (Linnaeus, 1759) & $\mathrm{FL}$ & $\mathrm{Y}$ & $1,2,3$ \\
\hline Biemnia sp. & & & 1 \\
\hline Chelonaplysilla noevus (Carter, 1876) & & & 1,2 \\
\hline Ciocalypta penicillus Bowerbank, 1864 & PA & $Y$ & 3 \\
\hline Clathria (Microciona) armata (Bowerbank, 1862) & EN & $Y$ & $1,2,3$ \\
\hline $\begin{array}{l}\text { Clathria (Microciona) atrasanguinea (Bowerbank, } \\
\text { 1862) }\end{array}$ & EN & $Y$ & 1,3 \\
\hline Clathria (Microciona) sp. & & & 2 \\
\hline Clathria (Microciona) spinarcus (Carter \& Hope, & & & 3 \\
\hline Clathria (Microciona) strepsitoxa (Hope, 1889) & & & 1,3 \\
\hline Clathrina clathrus (Schmidt, 1864) & & & 3 \\
\hline Clathrina coriacea (Montagu, 1818) & & & 3 \\
\hline Cliona celata (Grant, 1826) & $\mathrm{MA} / \mathrm{BU}$ & $Y$ & $1,2,3$ \\
\hline Crella rosea (Topsent, 1892) & & & 3 \\
\hline Crella sp. & & & 2 \\
\hline Dercitus bucklandi (Bowerbank, 1858) & & & 3 \\
\hline Desmacella annexa (Schmidt, 1870) & EN & $\mathrm{Y}$ & 2,3 \\
\hline Desmacella sp. & & & 2 \\
\hline Dysidea fragilis (Montagu, 1818) & MA & $\mathrm{Y}$ & $1,2,3$ \\
\hline Europon sp. & & & 2 \\
\hline Europon sp. 1 & & & 1 \\
\hline Europon sp. 2 & & & 1 \\
\hline Eurypon clavatum (Bowerbank, 1866) & & & 3 \\
\hline Eurypon clavigerum (Bowerbank, 1866) & & & 3 \\
\hline Eurypon major (Sarà \& Siribelli, 1960) & EN & $Y$ & $1,2,3$ \\
\hline Grantia compressa (Fabricius, 1780) & & & 3 \\
\hline Guancha lacunosa (Johnston, 1842) & $\mathrm{PE}$ & $Y$ & 1,2 \\
\hline $\begin{array}{l}\text { Halichondria (Halichondria) bowerbanki (Burton, } \\
\text { 1930) }\end{array}$ & & & 3 \\
\hline Halichondria (Halichondria) panicea (Pallas, 1766) & MA & $\mathrm{Y}$ & 1,3 \\
\hline Halichondria sp. & EN & $\mathrm{Y}$ & \\
\hline Haliclona (Gellius) angulata (Bowerbank, 1866) & & & 3 \\
\hline Haliclona (Halichoclona) fistulosa (Bowerbank, 1866) & $\mathrm{RE}$ & $\mathrm{Y}$ & $1,2,3$ \\
\hline Haliclona (Haliclona) oculata (Pallas, 1766) & MA/AR & $\mathrm{Y}$ & $1,2,3$ \\
\hline Haliclona (Haliclona) simulans (Johnston, 1842) & & & 3 \\
\hline Haliclona (Haliclona) urceolus (Rathke \& Vahl, 1806) & TU & $\mathrm{Y}$ & 3 \\
\hline Haliclona (Reniera) cinerea (Grant, 1826) & & $\mathrm{Y}$ & 1,3 \\
\hline
\end{tabular}




\begin{tabular}{|c|c|c|c|}
\hline Species & Morphology & $\begin{array}{l}\text { Present } \\
\text { study }\end{array}$ & $\begin{array}{l}\text { Other } \\
\text { studies }\end{array}$ \\
\hline $\begin{array}{l}\text { Haliclona (Rhizoniera) indistincta (Bowerbank, } \\
\text { 1866) }\end{array}$ & & & 3 \\
\hline Haliclona (Rhizoniera) rosea (Bowerbank, 1866) & & & 3 \\
\hline Haliclona (Rhizoniera) viscosa (Topsent, 1888) & MA & $\mathrm{Y}$ & 2,3 \\
\hline Haliclona sp. A. & & & 2 \\
\hline Haliclona sp. B. & & & 2 \\
\hline Halicnemia patera (Bowerbank, 1864) & EN & $\mathrm{Y}$ & $1,2,3$ \\
\hline Hemimycale columella (Bowerbank, 1874) & EN & $\mathrm{Y}$ & $1,2,3$ \\
\hline Hexadella racovitzai (Topsent, 1896) & & & 2 \\
\hline Homaxinella subdola (Bowerbank, 1866) & AR & $\mathrm{Y}$ & $1,2,3$ \\
\hline $\begin{array}{l}\text { Hymedesmia (Hymedesmia) jecusculum } \\
\text { (Bowerbank, 1866) }\end{array}$ & EN & $\mathrm{Y}$ & $1,2,3$ \\
\hline $\begin{array}{l}\text { Hymedesmia (Hymedesmia) pansa (Bowerbank, } \\
\text { 1882) }\end{array}$ & & & 3 \\
\hline $\begin{array}{l}\text { Hymedesmia (Hymedesmia) paupertas } \\
\text { (Bowerbank, 1866) }\end{array}$ & EN & $\mathrm{Y}$ & $1,2,3$ \\
\hline Hymedesmia (Stylopus) coriacea (Fristedt, 1885) & & & 2,3 \\
\hline Hymedesmia sp. A. & & & 2 \\
\hline Hymedesmia sp. B. & & & 2 \\
\hline Hymedesmia sp. C. & & & 2 \\
\hline Hymeniacidon perlevis (Montagu, 1818) & & & $1,2,3$ \\
\hline Hymeniacidon sp. & & & 3 \\
\hline Hymeraphia stellifera (Bowerbank, 1864) & EN & $\mathrm{Y}$ & 1,2 \\
\hline lophon hyndmani (Bowerbank, 1858) & & & 2,3 \\
\hline Iophon nigricans (Bowerbank, 1858) & & & 3 \\
\hline Leucosolenia botryoides (Ellis \& Solander, 1786) & & & 3 \\
\hline Leucosolenia complicata (Montagu, 1818) & & & 3 \\
\hline Leucosolenia sp. & & & 3 \\
\hline Mycale (Carmia) minima (Waller, 1880) & & & 2 \\
\hline Mycale sp. & & & 3 \\
\hline Myxilla (Myxilla) fimbriata (Bowerbank, 1866) & & & 3 \\
\hline Myxilla (Myxilla) incrustans (Johnston, 1842) & $\mathrm{MA}$ & $\mathrm{Y}$ & 3 \\
\hline Myxilla (Myxilla) rosacea (Lieberkühn, 1859) & & & 3 \\
\hline Myxilla sp. & & & 3 \\
\hline Ophlitaspongia papilla (Bowerbank, 1866) & & & 3 \\
\hline Oscarella lobularis (Schmidt, 1862) & & & 3 \\
\hline $\begin{array}{l}\text { Pachymatisma johnstonia (Bowerbank in } \\
\text { Johnston, 1842) }\end{array}$ & MA & $\mathrm{Y}$ & $1,2,3$ \\
\hline Paratimea constellata (Topsent, 1893) & EN & $\mathrm{Y}$ & 1,2 \\
\hline Phakellia ventilabrum (Linnaeus, 1767) & & & 3 \\
\hline Phorbas dives (Topsent, 1891) & EN & $\mathrm{Y}$ & 1,2 \\
\hline Phorbas fictitius (Bowerbank, 1866) & & & 2,3 \\
\hline Plocamionida ambigua (Bowerbank, 1866) & EN & $\mathrm{Y}$ & $1,2,3$ \\
\hline Polymastia boletiformis (Lamarck, 1815) & PA & $\mathrm{Y}$ & $1,2,3$ \\
\hline
\end{tabular}




\begin{tabular}{|c|c|c|c|}
\hline Species & Morphology & $\begin{array}{l}\text { Present } \\
\text { study }\end{array}$ & $\begin{array}{l}\text { Other } \\
\text { studies }\end{array}$ \\
\hline Polymastia mamillaris (Müller, 1806) & PA & $\mathrm{Y}$ & $1,2,3$ \\
\hline Polymastia penicillus (Montagu, 1818) & & & 2 \\
\hline Polymastia sp. 1 & $\mathrm{PA}$ & $\mathrm{Y}$ & 1,2 \\
\hline Polymastia sp. 2 & $\mathrm{PA}$ & $\mathrm{Y}$ & 1 \\
\hline Polymastia sp. B & & & 2 \\
\hline $\begin{array}{l}\text { Pseudosuberites sulphureus (Bean in Bowerbank, } \\
\text { 1866) }\end{array}$ & EN & $\mathrm{Y}$ & $1,2,3$ \\
\hline Raspaciona aculeata (Johnston, 1842) & AR & $\mathrm{Y}$ & $1,2,3$ \\
\hline $\begin{array}{l}\text { Raspailia (Clathriodendron) hispida (Montagu, } \\
\text { 1818) }\end{array}$ & AR & $\mathrm{Y}$ & $1,2,3$ \\
\hline Raspailia (Raspailia) ramosa (Montagu, 1818) & $A R$ & $\mathrm{Y}$ & $1,2,3$ \\
\hline $\begin{array}{llll}\text { Raspailia } & \text { (Raspailia) virgultosa } & \text { (Bowerbank, } \\
\text { 1866) } & & \\
\end{array}$ & & & 1 \\
\hline Raspailia/Stelligera juveniles & $A R$ & $\mathrm{Y}$ & 1 \\
\hline Sphaerotylus sp. A & & & 2 \\
\hline Sphaerotylus sp. B & & & 2 \\
\hline Stelletta grubii (Schmidt, 1862) & & & 3 \\
\hline Stelligera rigida (Montagu, 1818) & $A R$ & $\mathrm{Y}$ & 1,2 \\
\hline Stelligera stuposa (Montagu, 1818) & $A R$ & $\mathrm{Y}$ & 1,2 \\
\hline Stryphnus ponderosus (Bowerbank, 1866) & & & 3 \\
\hline Stylostichon sp. & EN & $\mathrm{Y}$ & 2 \\
\hline Suberites carnosus (Johnston, 1842) & $\mathrm{PE}$ & $\mathrm{Y}$ & $1,2,3$ \\
\hline Suberites ficus (Johnston, 1842) & & & 3 \\
\hline Sycon ciliatum (Fabricius, 1780) & & & 3 \\
\hline Sycon elegans (Bowerbank, 1845) & & & 3 \\
\hline Terpios fugax (Duchassaing \& Michelotti, 1864) & & & 3 \\
\hline Tethya aurantium (Pallas, 1766) & $\mathrm{GL}$ & $\mathrm{Y}$ & $1,2,3$ \\
\hline Tethya citrina (Sarà \& Melone, 1965) & $\mathrm{GL}$ & $\mathrm{Y}$ & 2 \\
\hline Tethyspira spinosa (Bowerbank, 1874) & MA & $\mathrm{Y}$ & $1,2,3$ \\
\hline Thymosia guernei (Topsent, 1895) & & & 2,3 \\
\hline Ulosa stuposa (Esper, 1794) & & & 3 \\
\hline 2 unidentified burrowing sp. & $\mathrm{BU}$ & $\mathrm{Y}$ & \\
\hline 2 unidentified massive sp. & MA & $\mathrm{Y}$ & \\
\hline 6 unidentified encrusting sp. & EN & $\mathrm{Y}$ & \\
\hline
\end{tabular}

The mean species richness $\mathrm{m}^{-2}$ is shown in Fig. 5.4. Mean species richness $( \pm \mathrm{S}$.E) was calculated as $25.32( \pm 1.02), 20.34( \pm 0.72)$ and $25.07( \pm 0.710)$ sponges $\mathrm{m}^{-2}$ at BG, DL and SH, respectively. No significant differences were found in species richness between sites using a permutational MANOVA. 


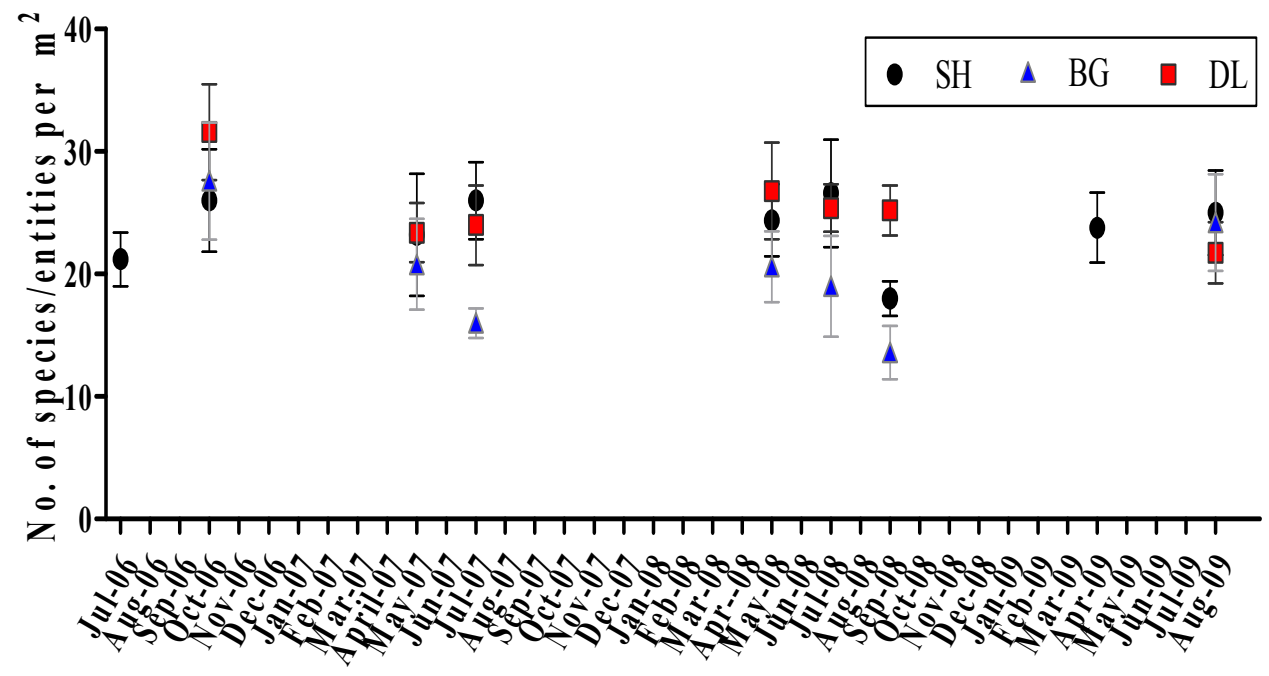

Fig5.4 Mean sponge species/entities per $\mathrm{m}-2 \pm \mathrm{SE}$ at the three Skomer sites (SH, BG, and DL) in spring, summer and autumn 2006-2009.

The mean abundance of sponges $\mathrm{m}^{-2}$ is shown in Fig. 5.5. The mean abundance of sponges per $\mathrm{m}^{-2}$ for each site $( \pm \mathrm{SE})$ was $160.1( \pm 18.84)$ sponges $\mathrm{m}^{-2}$ at $\mathrm{BG}$, $306.0( \pm 46.44)$ sponges $\mathrm{m}^{-2}$ at DL and $214.7( \pm 17.10)$ sponges $\mathrm{m}^{-2}$ at SH. Site (pseudo $\mathrm{F}=23.151 \mathrm{p}=0.0005$ ) and season nested in year (pseudo $\mathrm{F}=8.0788 \mathrm{p}=$ 0.007 ) were significant factors explaining the differences in sponge abundance using a permutational MANOVA.

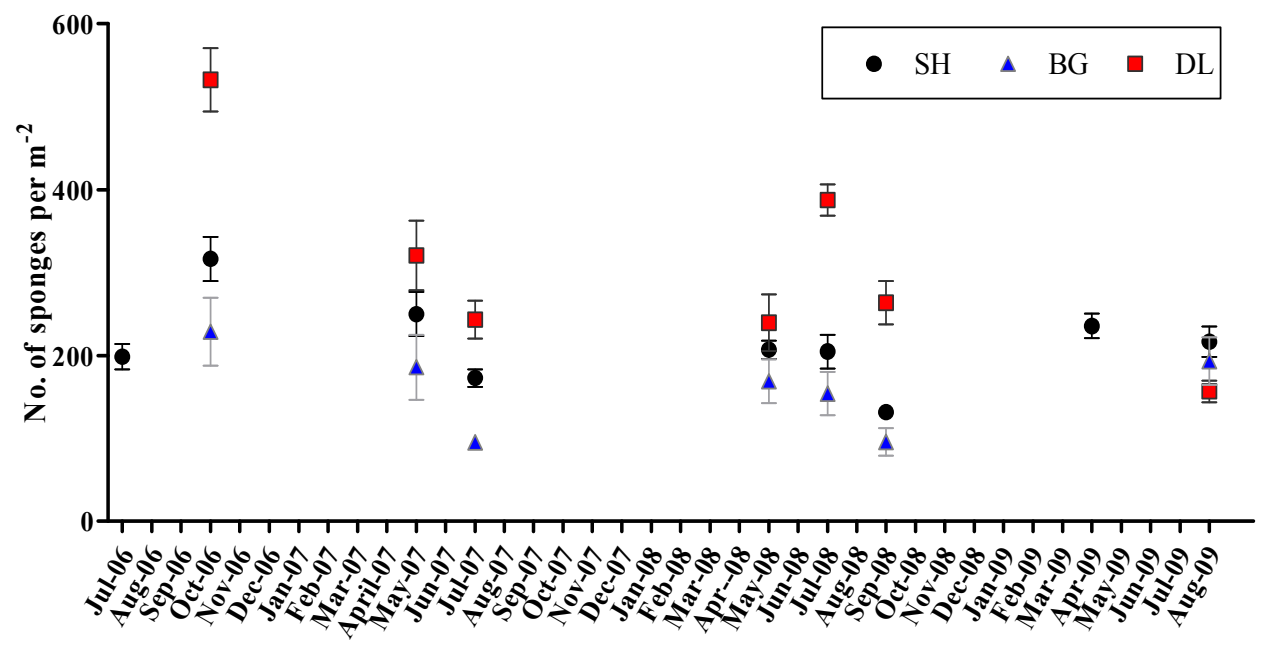

Figfigure 5.5 Mean No. of sponges per $\mathrm{m}-2$ \pm S.E at the three sites (SH, BG, and DL) in spring, summer and autumn 2006-2009. 
The MDS plot for the species assemblage data shows that there differences in sponge assemblage between sites and seasons (Fig. 5.6).

PERMDISP was used to to test for beta diversity between seasons across all sites. There was a significant difference between seasons $P($ perm $)=0.018$ Pseudo F: 5.0561. Site is also shown to be a significant factor using PERMDISP to test for beta diversity between sites explaining the high variability in sponge diversity even though the sites were only a couple of hundred metres apart, $\mathrm{P}($ perm $)=0.001$ Pseudo F: 11.039 .

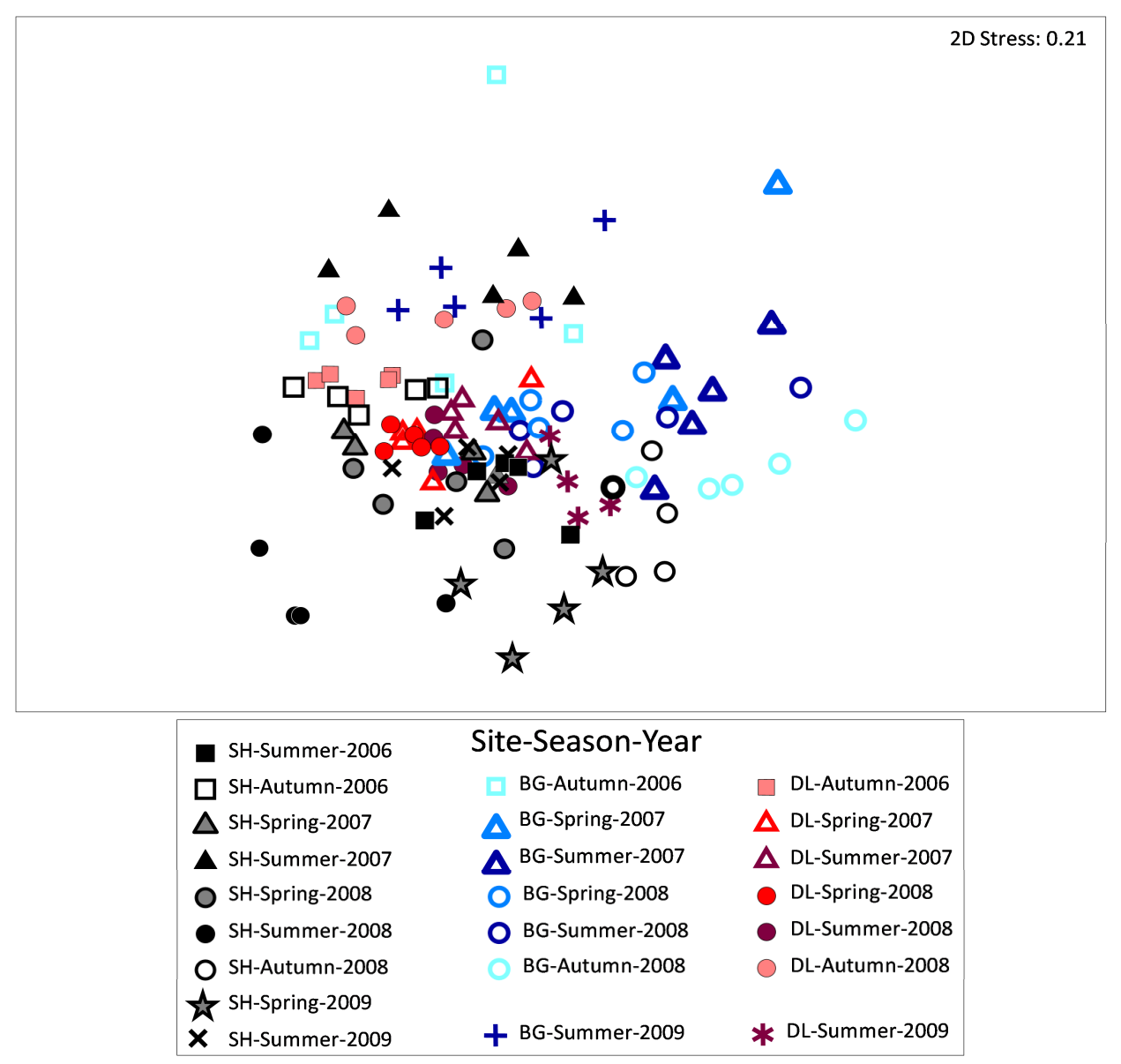

Fig. 5.6 Multi-dimensional scaling (MDS) plot of sponge assemblages based on the species data across the three sites (SH, BG \& DL) at Skomer MNR showing the spatial, seasonal and annual variability. 
The significant factors for explaining species assemblage changes using the PERMANOVA model were site, quadrat (nested in site), season (nested in year), and the interaction between site*season (nested in year)(Table 5.4).

Table 5.4 PERMANOVA results of the species assemblage data. Significant factor values are marked in bold. Unique permutations were observed out of 1000.

\begin{tabular}{|c|c|c|c|c|c|c|}
\hline Source & $\begin{array}{l}\text { Degrees } \\
\text { of } \\
\text { freedom }\end{array}$ & $\begin{array}{l}\text { Sums of } \\
\text { squares }\end{array}$ & $\begin{array}{l}\text { Expected } \\
\text { mean of } \\
\text { squares }\end{array}$ & Pseudo-F & $P($ perm $)$ & $\begin{array}{l}\text { Unique } \\
\text { perms }\end{array}$ \\
\hline Site & 2 & 9911.9 & 4956 & 2.3749 & 0.034 & 999 \\
\hline Year & 3 & 5133.6 & 1711.2 & 0.69761 & 0.956 & 999 \\
\hline Quadrat(Site) & 12 & 11931 & 994.22 & 3.482 & 0.001 & 997 \\
\hline Season(Year) & 5 & 13291 & 2658.1 & 2.2336 & 0.007 & 998 \\
\hline Site*Year & 6 & 8042.2 & 1340.4 & 1.1427 & 0.2 & 997 \\
\hline Site*Season(Year) & 6 & 7021.5 & 1170.2 & 4.1211 & 0.001 & 995 \\
\hline Quadrat(Site)*Year & 35 & 9677.1 & 276.49 & 0.97368 & 0.609 & 996 \\
\hline Residual & 44 & 12494 & 283.97 & & & \\
\hline Total & 113 & 77502 & & & & \\
\hline
\end{tabular}

SIMPER analysis showed that the three O.T.U's that were the most consistent in their abundance within each of the sites (had the highest percentage contribution to the similarity within a site) were: Polymastia boletiformis (16.30\% in BG; $9.28 \%$ in DL and $11.01 \%$ in SH), Stelligera stuposa (10.84\% in BG; $8.63 \%$ in DL and $11.29 \%$ in SH) and Raspailia/Stelligera juveniles (10.18\% in BG; $9.17 \%$ in DL and $9.72 \%$ in $\mathrm{SH}$ ). The main two species explaining the differences and therefore most variability between the sites were Hymeraphia stellifera (5.59\% between BG and SH; 7.38\% between BG and DL and 5.33\% between DL and SH) and Halicnemia patera (4.60\% between BG and SH; 5.51\% between BG and DL and $4.43 \%$ between DL and SH). Eighteen to twenty species were needed to explain $60 \%$ of the dissimilarity between sites (See Appendix 5.6 for the SIMPER Data). 
The main species explaining the seasonal changes in the Skomer MNR according to the SIMPER analysis were Hymeraphia stellifera, Halicnemia patera, Haliclona (Haliclona) oculata and Haliclona (Haliclona) urceolus. The mean abundances per quadrat across all three sites for the four species are shown in Fig. 5.7. Hymeraphia stellifera and Halicnemia patera showed similar seasonal fluctuations with highest abundances in October 2006 of 69 and 34 sponges per $1 \mathrm{~m}^{2}$ respectively. There were $25 \%$ fewer Hymeraphia stellifera and half the number of Halicnemia patera by the following spring. The abundance of both these species continued to decline in July 2007 to about $15 \%$ of the October 2006 abundances for both species. By spring 2008 both species had returned to similar abundances as spring 2007. The numbers for April 2009 were lower than the previous spring abundances but increased slightly by the following August. H. oculata had a mean density of $<4$ individuals per $1 \mathrm{~m}^{2}$ until September 2008 when the mean number of sponges rises to over 11 sponges per $1 \mathrm{~m}^{2}$. In April 2009 there were a similar number of $H$. oculata present with 10 sponges per $1 \mathrm{~m}^{2}$, which declines to 7 sponges per $1 \mathrm{~m}^{2}$ by August 2009. $\mathrm{H}$. urceolus from 2006-2008 showed a slight increase in abundance every spring, however, in April 2009 there was more than a 10-fold increase in abundance compared to the previous spring, increasing from less than 5 sponges per $1 \mathrm{~m}^{2}$ to more the 56 sponges $\mathrm{m}^{2}$. However by August 2009 the numbers were back to the original low densities of less than 2 sponges $\mathrm{m}^{-2}$. 


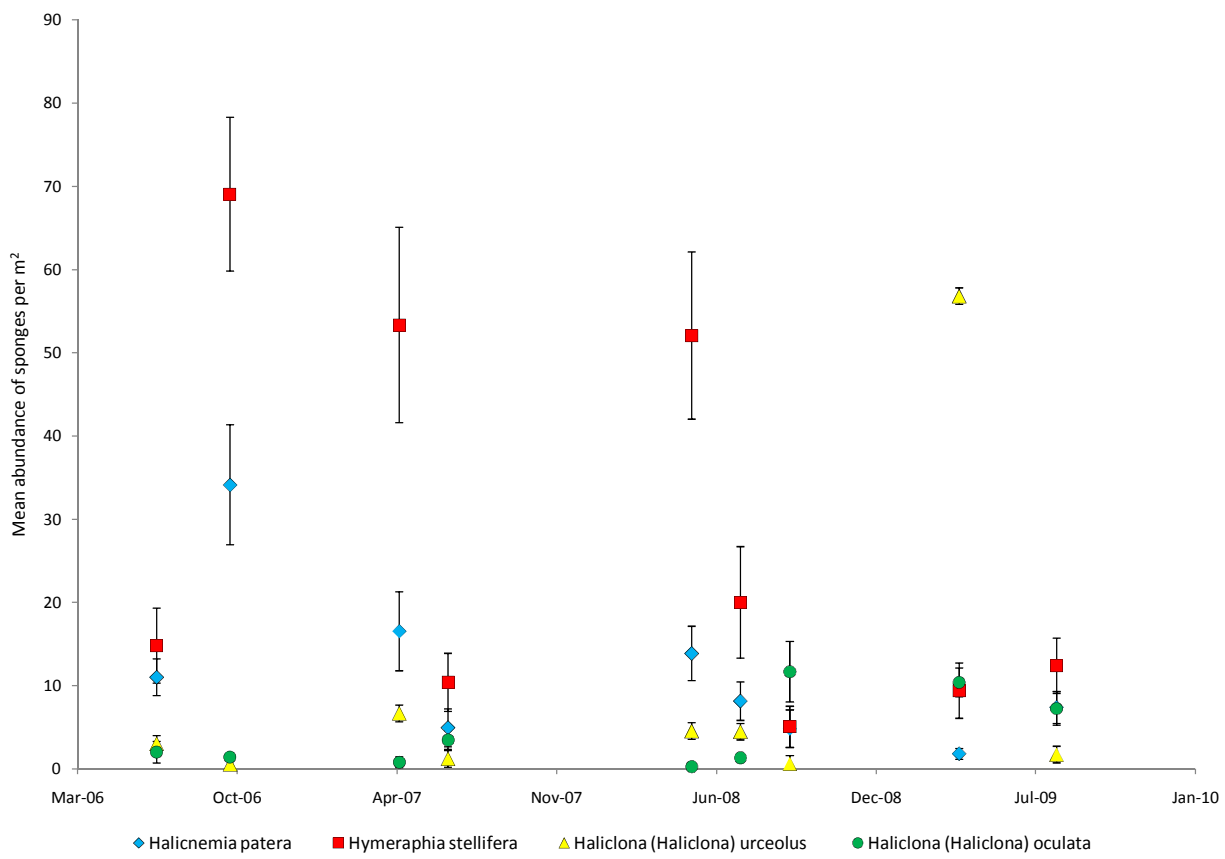

Figure 5.7 Seasonal mean abundances ( \pm S.E) of Hymeraphia stellifera, Halicnemia patera, Haliclona (Haliclona) oculata and Haliclona (Haliclona) urceolus within Skomer MNR. 


\subsubsection{MORPHOLOGICAL ASSEMBLAGE DATA}

Ten morphological types were recorded across the three sites surveyed. Mean morphological richness $\left( \pm\right.$ S.E) was calculated as $8.11 \pm 0.56 \mathrm{~m}^{-2}$ at $\mathrm{BG}, 8.66 \pm 0.40$ sponges $\mathrm{m}^{-2}$ at DL and $7.82 \pm 0.71$ sponges per $\mathrm{m}^{-2}$ at $\mathrm{SH}$. There were no significant differences in the number of morphological types present between sites of season when tested using a permutational MANOVA. The MDS plot for the morphological assemblage data shows that site and season influenced assemblage structure (Fig. 5.8).

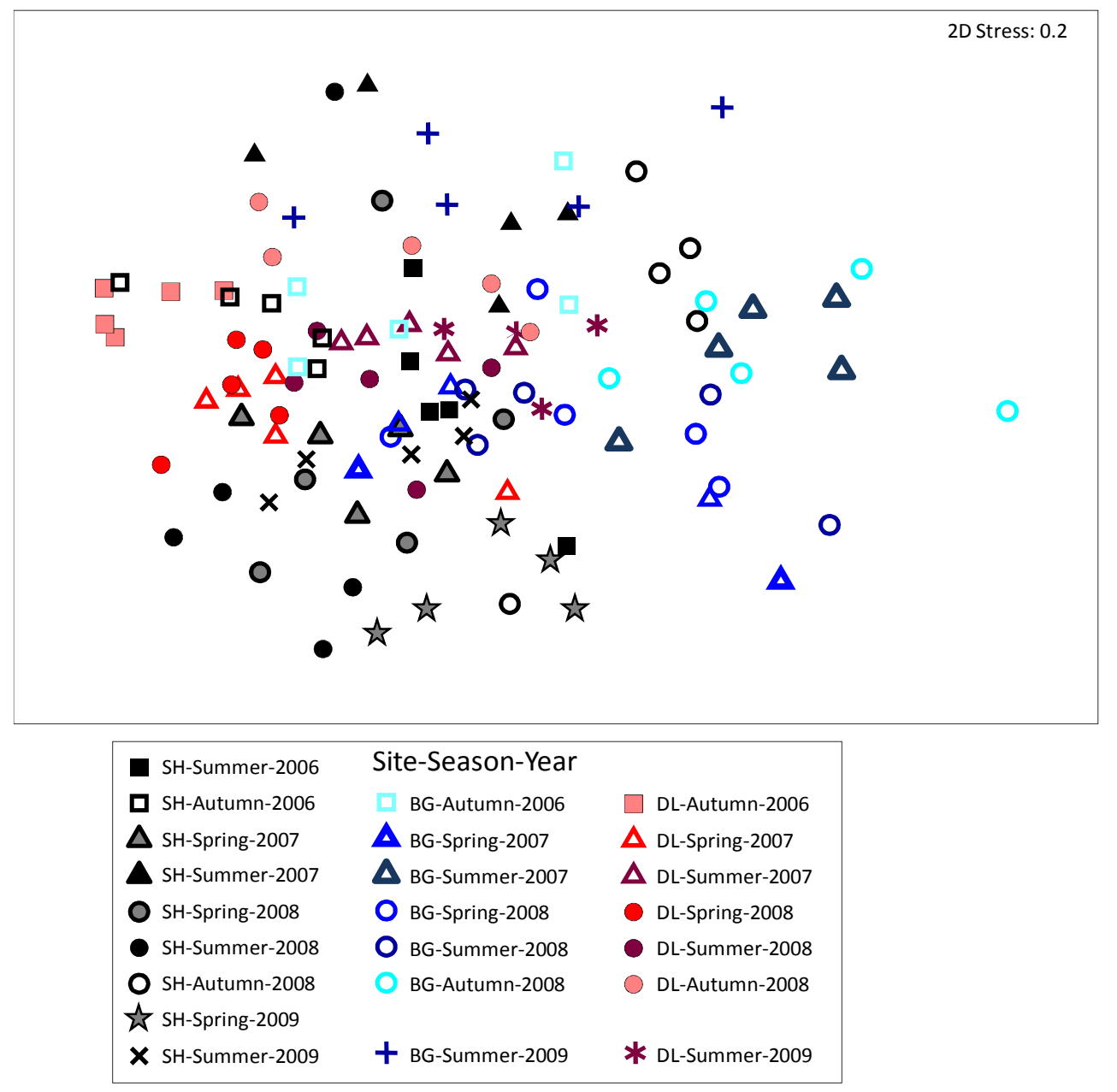

Figure 5.8 Multi-dimensional scaling (MDS) plot of sponge assemblages based on the morphological data at the three sites (SH, BG, and DL).

PERMDISP was used to to test for beta diversity between sites across all seasons and years. There was a significant difference between sites $P($ perm $)=$ 
0.006 Pseudo F: 6.2636. Season and year were not significant factors for the morphological assemblages using PERMDISP to test for beta diversity.

The PERMANOVA model confirmed that spatial and temporal shifts in morphological assemblage data at the site and quadrat level were significant (quadrat $\mathrm{p} \leq 0.01$, site $\mathrm{p} \leq 0.5$ ). Seasonal data were significant to at least $\mathrm{p} \leq 0.5$ and site interacted significantly with the seasons at $p \leq 0.1$ for both the morphological and species assemblage data (Table 5.5). This suggests that seasonality has different effects on each of the site. The annual time scale was not significant using all the data or for assemblage data from a single season or site. The same factors were significant as those for the species assemblage data.

Table 5.5 PERMANOVA results of the morphology assemblage data. Significant factor values are marked in bold text. Unique permutations observed out of 1000.

\begin{tabular}{|c|c|c|c|c|c|c|}
\hline Source & $\begin{array}{c}\text { Degrees } \\
\text { of } \\
\text { freedom }\end{array}$ & $\begin{array}{l}\text { Sums of } \\
\text { squares }\end{array}$ & $\begin{array}{c}\text { Expected } \\
\text { mean of } \\
\text { squares }\end{array}$ & Pseudo-F & $\mathrm{P}($ perm $)$ & $\begin{array}{l}\text { Unique } \\
\text { perms }\end{array}$ \\
\hline Site & 2 & 4456 & 2228 & 2.947 & 0.037 & 997 \\
\hline Year & 3 & 1328.8 & 442.93 & 0.52397 & 0.943 & 998 \\
\hline Quadrat(Site) & 12 & 6354.1 & 529.51 & 7.6711 & 0.001 & 997 \\
\hline Season(Year) & 5 & 5521.7 & 1104.3 & 3.1656 & 0.016 & 998 \\
\hline Site*Year & 6 & 2754.4 & 459.07 & 1.3021 & 0.176 & 997 \\
\hline Site*Season(Year) & 6 & 2093.2 & 348.86 & 5.054 & 0.001 & 996 \\
\hline Quadrat(Site)* Year & 35 & 2290.7 & 65.448 & 0.94815 & 0.586 & 999 \\
\hline Residual & 44 & 3037.2 & 69.027 & & & \\
\hline Total & 113 & 33204 & & & & \\
\hline
\end{tabular}

SIMPER analysis of the morphological assemblage data showed that the arborescent sponges were the most consistent in abundance within a season with contributions of $24.24 \%, 28.87 \%$ and $29.28 \%$ across spring, summer and autumn, respectively. Papillate sponges were the second most consistent group in terms of abundance in summer and autumn with $20.96 \%$ and $22.15 \%$, and the third most important in spring with $21.09 \%$. Encrusting sponges were the second largest contributor to the similarities within sites in spring with $21.75 \%$ 
and third most in summer and autumn with $18.73 \%$ and $18.67 \%$, respectively. These three morphologies are responsible for $65 \%$ of the similarities within each season. The morphologies contributing the most to the differences between seasons were the encrusting sponges $(24.22 \%, 29.17 \%$ and $30.51 \%$ for spring and summer, spring and autumn, and summer and autumn, respectively) and arborescent sponges (24.22\%, 29.17\% and 30.51\% for spring and summer, spring and autumn, and summer and autumn, respectively). Tubular sponges were the third largest contributors to differences between spring and summer (13.08\%), spring and autumn (14.24\%), while pedunculate sponges were the third highest contributor for summer and autumn with (See Appendix 5.1 for full SIMPER analysis).

\subsubsection{CORRELATIONS BETWEEN THE SPECIES AND MORPHOLOGICAL DATA}

The changes in species and morphological assemblage data were compared within each site separately over time (Fig 5.9). All the sites showed seasonal oscillations, which were similar for the morphological and species data sets. There were significant changes in the species and morphological assemblages across all three sites between summer 2008 and autumn 2008. After the change in the assemblage at Spongy Hillocks the assemblage returned to its previous morphological and species assemblage structure by summer 2009. Broad Gulley also returned to an assemblage structure similar to the first sampling period in autumn 2006, so summer 2009 represented a considerable change in comparision to other seasonal changes. Dog Leg remained relatively stable until the change in autumn 2008 and then in summer 2009 this site appeared to have another morphological and species assemblage change. 

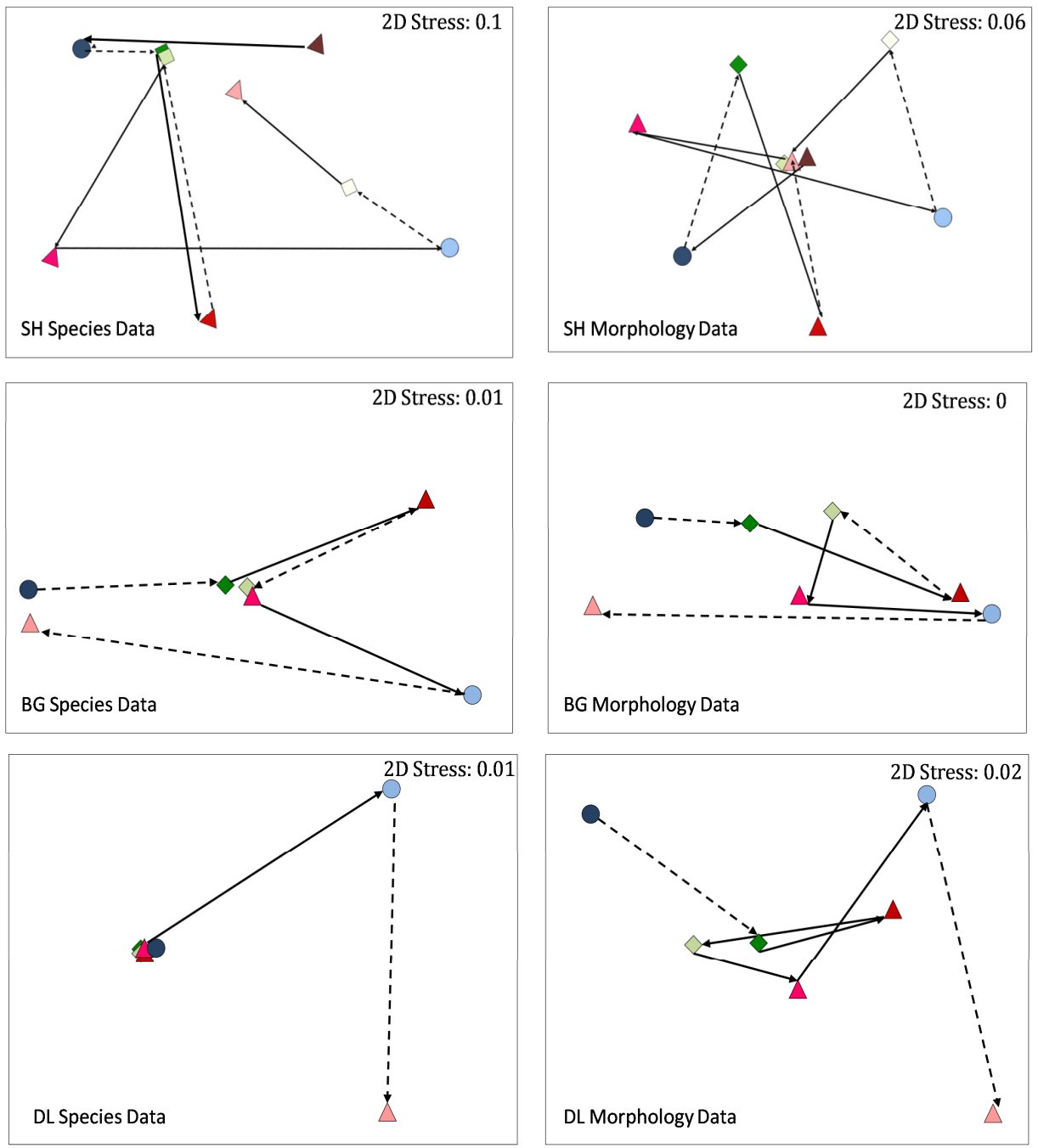

\begin{tabular}{|llll}
$\Delta$ Summer-2006 & Autumn-2006 & $\diamond$ Spring-2007 & $\Delta$ Summer-2007 $\diamond$ Spring-2008 \\
$\Delta$ Summer-2008 & OAutumn-2008 & $\diamond$ Spring-2009 & $\Delta$ Summer-2009
\end{tabular}

Fig. 5.9 MDS plots of the seasonal shifts in species and morphological assemblages in Skomer MNR. The MDS plots on the left show the species data and the MDS plots on the right are from the morphological data. The top two plots are the Spongy Hillocks data sets, the middle plots are the Broad Gulley data sets and the bottom plots are the Dog Leg data sets. The solid arrows signify a one season change and the dotted arrows signify that there is a gap of a season or more between the two data points.

The RELATE function was used to measure how closely correlated the morphological and the species assemblage data were using Spearmans $\rho$. The species and morphological data resemblance matrices correlated significantly $0.01 \%$ using 9999 permutations for the combined data sets or within the individual sites (Table 5.6). 
Table 5.6 Spearman's rank correlations between the morphological and species data sets.

\begin{tabular}{|l|l|l|}
\hline Site & Spearmans Rank Coefficient & Significance \\
\hline All Skomer & 0.772 & 0.01 \\
\hline Spongy Hillocks & 0.712 & 0.01 \\
\hline Broad Gulley & 0.768 & 0.01 \\
\hline Dogs Leg & 0.67 & 0.01 \\
\hline
\end{tabular}

\subsubsection{ENVIRONMENTAL DATA CORRELATIONS}

The environmental factors that correlated with the change in species assemblage composition at Skomer were the combination of water temperature, maximum air temperature, net radiation per month and maximum wind gust speed or all of the above swapping the mean water temperature for mean air temperature. Both of the above combinations had a Rho value of 0.26 , which were significant at $0.01 \%$ (9999 permutations). The shifts in morphological assemblage composition correlated with the water temperature, maximum air temperature net radiation and maximum wind gust speed, which were the same as one of the highest correlations for explaining species assemblage shifts. A combination of Sechi disc readings and maximum air temperature also correlated with the morphological data and both models had a Rho value of 0.17 which was significant to $0.01 \%$ (9999 permutations).

Over the study period from 2006-2009, 2006 had the coolest spring and summer mean seawater temperatures (Fig. 5.10). 2006 also had the warmest temperatures in autumn which led onto the warmest spring and early summer in 2007. 2008 and 2009 both had the highest temperatures for August and September and spring temperatures similar to 2006. 2008 had the coolest autumn period during the study. 


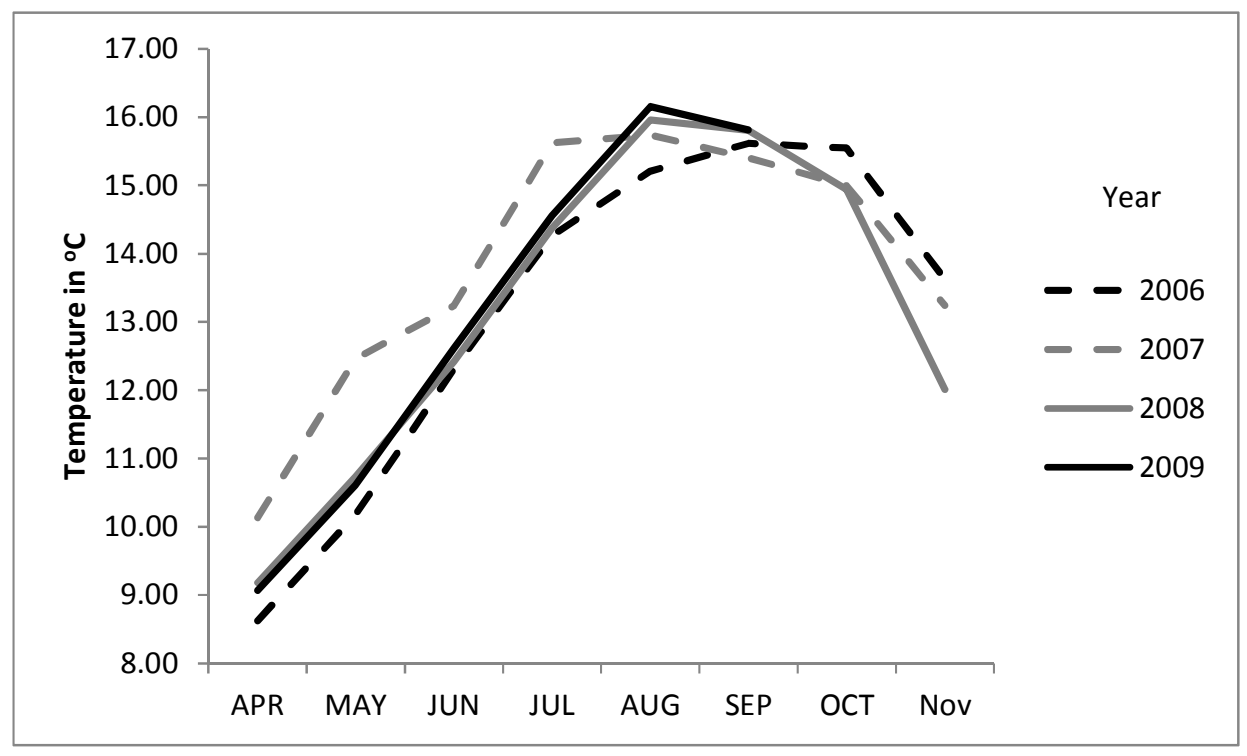

Fig. 5.10 Variation in Seawater temperature from 2006-2009.

The air temperature (maximum, mean and minimum) records cover the years 1993-2009 (Fig. 5.11). The summer of 2006 had the hottest maximum temperature since the summer of 1995 , and the hottest mean since the summer of 1997 . The winter of $2006 / 7$ was the mildest since the winter of $1998 / 9$, however there were no records for winter from the previous year (2005/6). Over the study period the winter means were colder each year from 2006/72008/9 while the mean summer temperatures were similar for summers 20079. The maximum summer air temperatures were hottest in 2006 and coolest in 2007.

The monthly net radiation data were only available from January 2007 till September 2009 (Fig. 5.12). The highest net radiation was in June 2008 and lowest value was in December 2008. The months of May - August for 2007 and 2009 were warmer on average overall compared to May-August 2008 because they were consistently above 100 rather than a sharp peak as in the case for the month of July in 2008. 


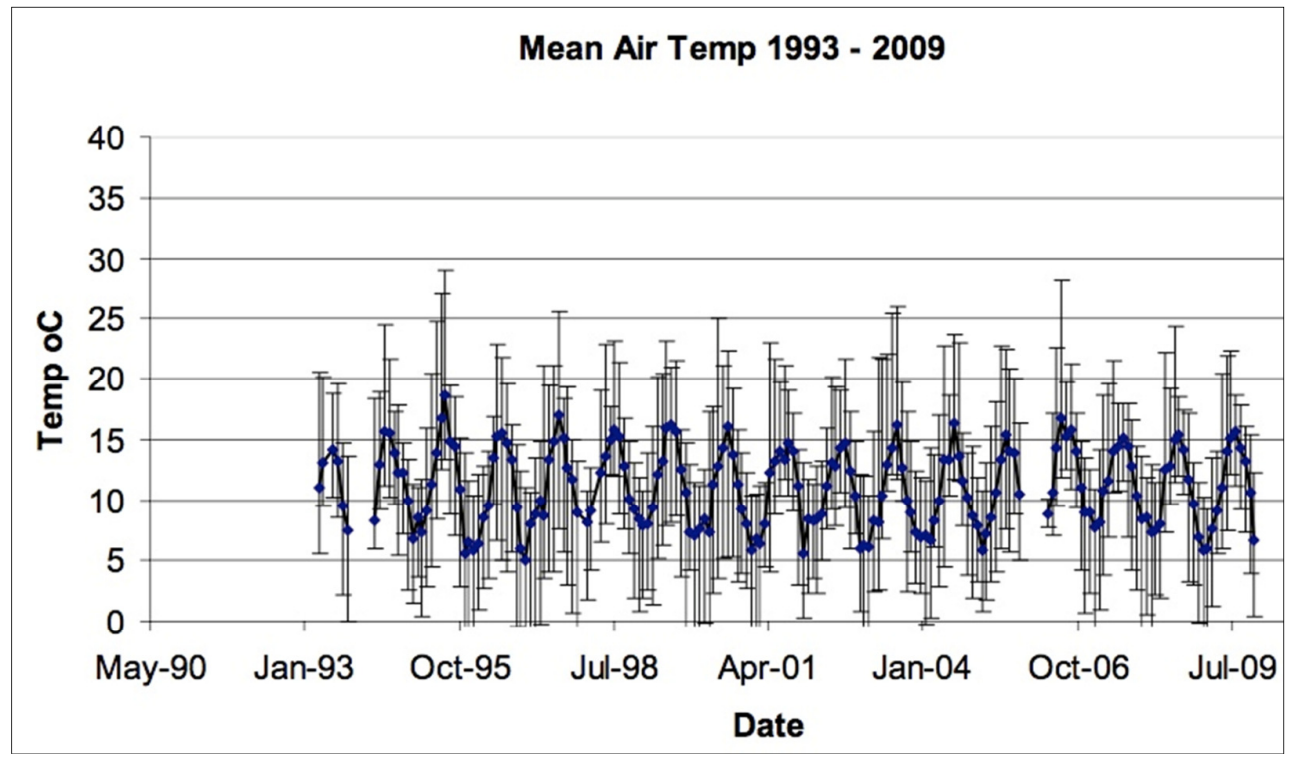

Figure 5.11 Variation in air temperature (mean, maximum and minimum) from 1993-2009. The graph was taken from CCW report 2009/10 (Lock et al., 2010a).

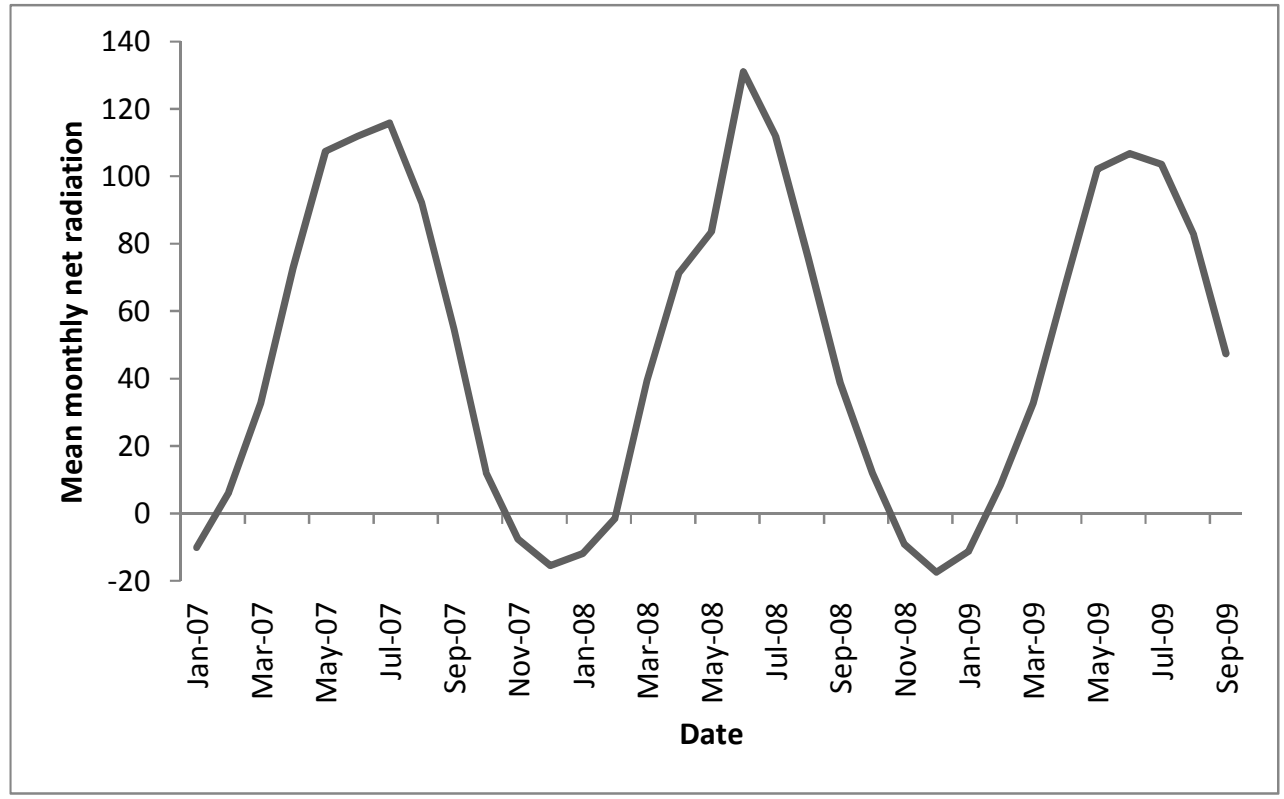

Figure 5.12 Variation in net radiation per month from 2007-2009. The graph was created using CCW environmental data (Burton et al., 2008; Lock et al., 2010a). 
The maximum wind gust speed data were only available for January 2007 till September 2009 (Fig. 5.13). The highest gusts were generally over winter time with gusts above 40m/sec in January 2007 and December 2008. The lowest maximum gust speeds (under $25 \mathrm{~m} / \mathrm{sec}$ ) occurred in April and August 2007, July 2008 and July 2009.

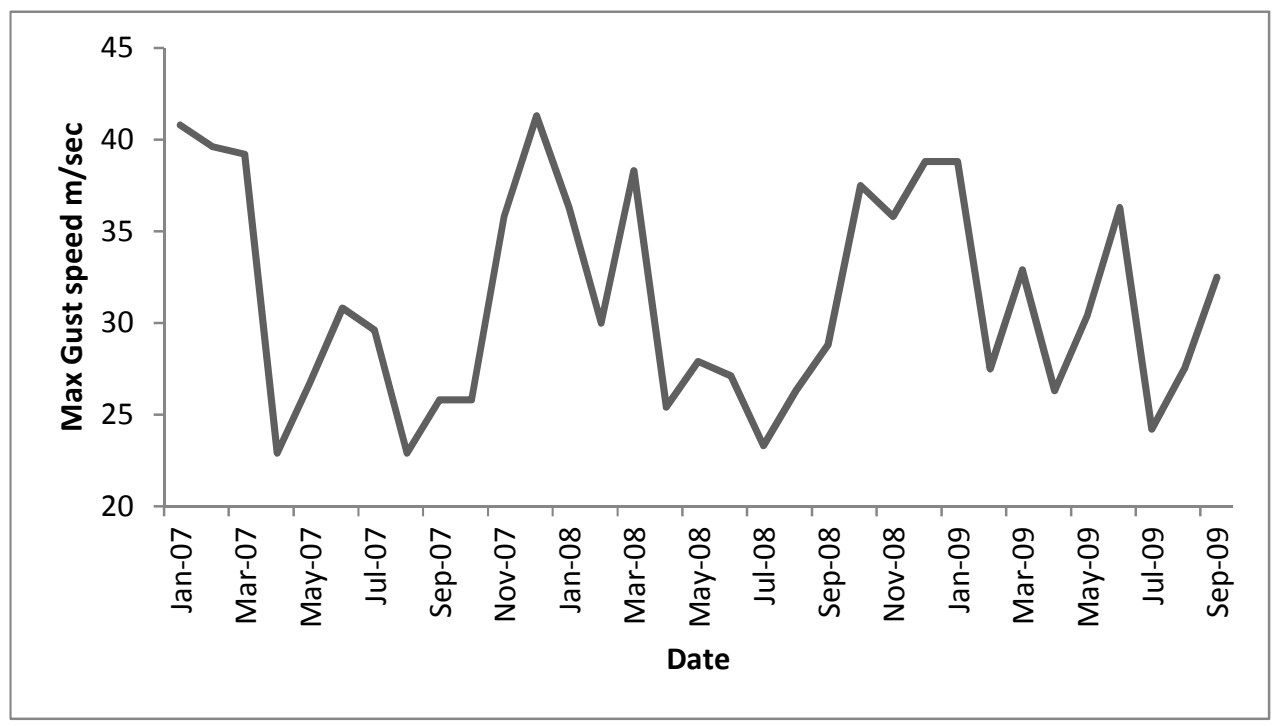

Figure 5.13 Monthly maximum wind gust speed $\mathrm{m} / \mathrm{sec}$ from 2007-2009. The graph was created using CCW environmental data.

The sechi disc water visibility data is available for the months of April till October in 2006-2009 (Fig. 5.14). The best visibility each year occurred in August in 2006, July in 2007, June and July in 2008 and June in 2009. In 2006, 2007, and 2008 the visibility dropped off in spring and autumn while in 2009 the visibility range was narrower remaining at almost $6 \mathrm{~m}$ or higher for all of the records. 
Chapter 5 Spatial and temporal variability of sponge species and morphological diversity

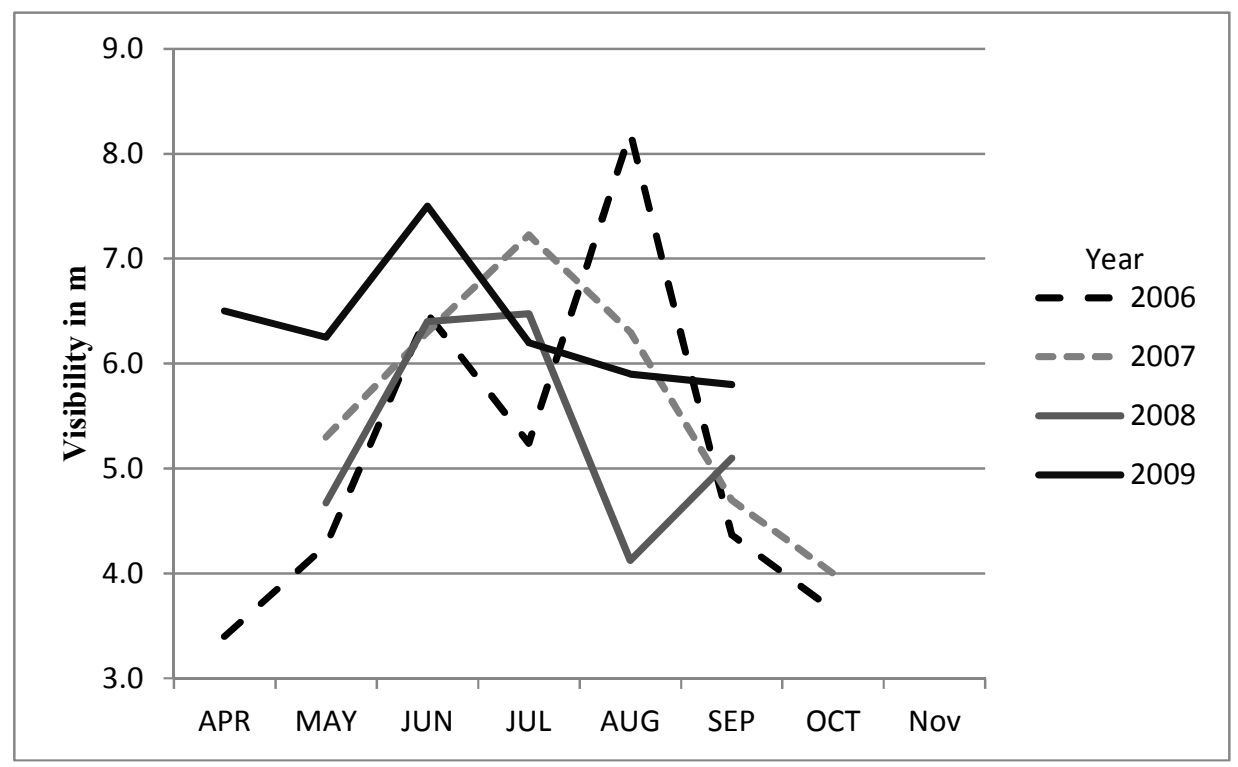

Fig. 5.14 Variation in water visibility using the sechi disc from 2006- 2009. The graph was created using CCW environmental data. 


\subsection{DISCUSSION}

\subsubsection{PATTERNS OF SEASONAL VARIABILITY IN THE SPONGE SPECIES ASSEMBLAGES WITHIN} SKOMER MNR

Fifty-nine sponge species/entities were found within the $15 \mathrm{~m}^{2}$ over the four years of surveying at Skomer MMR. This was almost $50 \%$ of the known biodiversity from the MNR and similar to the 57 sponge species recorded from the $5 \mathrm{~m}^{2}$ surveyed by Bell (2006) in the Thorn Rock area. This suggests that Skomer has a patchy distribution of species as half the known species within the local area were sampled. The mean species richness did not vary significantly between seasons at any of the sites, however, site and season were important factors explaining the change in the abundance of sponges. From 2006-2008, DL had the highest abundance of sponges at each sampling point, BG had the lowest and SH was intermediate. This changed by August 2009, where DL had the lowest abundance of sponges. Sponge abundance can increase through the processes of new recruits settling, budding and fission of the sponges present in the area already. Sponge abundance (number of patches) can decrease through mortality, predation or fusion of patches of sponges of the same species.

The main species explaining the seasonal species assemblage changes were Hymeraphia stellifera, Halicnemia patera, Haliclona (Haliclona) oculata and Haliclona (Haliclona) urceolus. Hymeraphia stellifera and Halicnemia patera were found across all three sites and seasons and showed similar patterns of high abundances in autumn with a small decline by the subsequent spring, then low abundances in summer before a steep increase in autumn. The ecology of these specific species has not been previously investigated, however, from their surface morphologies they are well adapted to living in highly sedimented such as Skomer MNR and filtering the interstitial water, which is beneficial because it is rich in nutrients and food particles (Ilan \& Abelson, 1995). Hymeraphia stellifera is a very thin encrusting sponge that has small regularly raised oscular conules. It is normally covered in a layer of silt but often the conules were clear even if the rest of the surface was not. Halicnemia patera is 
also a thin encrusting sponge that forms small patches up to $2 \mathrm{~mm}$ thick. It has a conulose surface that has bundles of long spicules facing in one direction giving it a windswept appearance and it exudes slime when damaged.

Haliclona (Haliclona) oculata was present at relatively low abundance; however, there was a tenfold increase between July and September 2008. $H$. oculata is known to produce larvae from July till November so this may have been a reproductive event followed by rapid growth (Wapstra \& Soest, 1987). H. oculata in the Netherlands have been shown to have an average linear growth rate of 0.015 day $^{-1}$ so it could be possible for small sponges to become visible within the quadrat photographs within this time period (Koopmans \& Wijffels, 2008). H. oculata can grow in a variety of morphologies from thickly encrusting and cushions to massive and arborescent. Massive sponges resist damage better then arborescent sponges, and H. oculata can change its growth form in response to changing environmental conditions (Kaandorp, 1995). However, at Skomer most of the forms were arborescent, which might be advantageous in the highly sedimented conditions as the amount of sediment falling per unit area on the sponge is much lower than for other morphologies (Chappell, 1980). H. oculata is also one of the few sponges that has been documented as being susceptible to disease (although that is likely to be through a lack of documentation not lack of diseases) in the form of brown decaying patches that were suspected to be of microbial origin (detected in $\mathrm{N}$. Wales) (Webster, 2007). When arborescent sponges are affected by a disease they often loose branches, however, this is also a defensive mechanism which may prevent the infection spreading to other healthy parts of the sponge (Wulff, 2006c). H. oculata could have been suffering from a disease prior to the study period and then the disease cleared by summer 2008. 


\subsubsection{PATTERNS OF SEASONAL VARIABILITY IN THE SPONGE MORPHOLOGICAL ASSEMBLAGES IN} SKOMER MNR

Encrusting species and arborescent species were the main drivers of morphological change. Encrusting sponges are known to undergo fission and fusion over time, which would increase and decrease the abundance of sponge patches (Ayling, 1983b; Pansini \& Pronzato, 1985; Tanaka, 2002; Blanquer et al., 2009). Fusion normally only happens between fragments that are not only the same species, but are also genetically identical (van de Vyver, 1975; Amano, 1990; Blanquer et al., 2009). The fission and fusion of encrusting sponges has been related to random events (Ayling, 1983b; Tanaka, 2002) and/or seasonality (Blanquer et al., 2008). There were seasonal shifts in the species assemblages in the Skomer MNR so stochastic processes were unlikely to be responsible for the changes in the sponge assemblages. Encrusting sponges have a lower profile and therefore are unlikely to be removed easily when subjected to forces or damaged as easily as massive or arborescent species by storms, however, they might have low regeneration rates if damaged, for example, by grazing due to no underlying tissue to support the process (Wulff, 2006b). Encrusting sponges can be vulnerable to smothering, as although some species can take advantage of the interstitial water, very high sedimentation levels may block the ostia of other species preventing filtration processes and leading to a turnover in the assemblage structure as the encrusting sponges better adapted to sedimented waters survive and those less well adapted regress or die off.

Some arborescent sponges are known to break easily, however, others can be very flexible. In addition, when branches are lost the original sponge heals relatively quickly and the fragments can reattach to the substrate and continue to grow (Wulff, 2006b). Fragments are less likely to reattach in the Skomer MNR because of the fast tidal currents passing through the area which might carry them away to deeper soft sediment environments. However, these currents could also bring new fragments into the area during the frequent winter storms (Bell et al., 2006). Reattachment of fragments was not observed within the quadrats monitored, however, there were lots of Raspailia/Stelligera 
juveniles therefore there was likely to have been recruitment events during the three year study period.

Tubular sponges were responsible for causing changes in morphological assemblage composition before and after spring 2009. Tubular morphology sponges were all of the species $H$. urceolus and were present in low densities throughout the year. $H$. urceolus are known to be efficient at passively preventing settlement of sediment through their morphology so they could have gained a competitive advantage over other species during times of heavy sedimentation by preventing sediment building up on their surface blocking their ostia (Bell, 2004). However, in spring 2009 there was a significant increase in numbers from less than 10 per m$^{-2}$ to between 35-100 per m-2 of $H$. urceolus sponges in the Spongy Hillocks site quadrats. The visibility was much higher in spring 2009 (over 6 m compared with 4-5 m) and therefore the sediment load would probably have been reduced in the water, especially as there was stronger wind in spring 2009 compared to spring 2007 and 2008 preventing the sediment settling. As little is known about the ecology of this sponge species it is hard to predict why this increase in numbers occurred, however, it is a small tubular sponge (1-2 cm high) so there is the potential for a high number of sponges to have recruited and settled to the Spongy Hillocks site between autumn 2008 and spring 2009, however, by summer 2009 they were back to their low numbers $(<5$ individuals per quadrat) again. The return to low numbers could be due to a storm event, however at $12 \mathrm{~m}$ depth the swell is not likely to have a significant impact, even though the sponges were only attached by a small basal disk to the rock and therefore were easy to remove. $H$. urceolus could have declined due to a lack of food availability as the water visibility remained clearer than previous years throughout spring and summer 'and therefore the sponge tissues could have regressed. Also as the increases in numbers were all within one site they could have been removed by physical disturbance such as a boat anchor or similar. One of the massive specimens of Cliona celata had half of its mass removed between spring and summer 2007 therefore large disturbances do happen within the MNR and could easily damage a small area (pers. obs). 
Abiotic factors are thought to be the main drivers of changes in temperate sponge assemblages. (Bell \& Barnes, 2000b, a) Predation, for example by spongivorous fish or nudibranchs as well as the mechanisms used by sponges to deter predators, can play a role in shaping sponge community structure on temperate reefs although at it has been reported at a lower intensity than in tropical areas (Ruzicka \& Gleason, 2008; Ruzicka \& Gleason, 2009). There is no information on spongivorous fish in Skomer MNR and no fish feeding on sponges was observed during surveying. There are however, over seventy nudibranch species recorded from Skomer MNR with thirty recorded from the Thorn Rock area where the sponge survey sites are located (Lock et al., 2010b). A nudibranchs diet is often specific to one species/genus. There are three known species within Skomer MNR that only feed on specific sponge species. One of these species Jorunna tomentosa is known to feed on Haliclona spp. (Lock et al., 2010b). Therefore it could have consumed H. urceolus as it t has been found within Skomer MNR although not recorded within the Thorn Rock area There are many more nudibranchs within the MNR with unknown dietary preferences and these could also have had an impact on the abundance of $H$. urceolus.

Sponge disease epidemics can also have serious long-term effects on sponge assemblages causing up to 95\% mortality of some species (Galtsoff, 1942; Vacelet et al., 1994; Cervino et al., 2006). Disease is not always easy to detect as it can develop hidden within the main sponge body and dead sponges are not easy to detect as the bare skeletal fibres rapidly disintegrate (Webster, 2007). There is also a lack of knowledge of sponge diseases in general and therefore without sampling sponges that are affected then it is difficult to predict that disease was the causative factor for the decline.

\subsubsection{THE CORRELATIONS OF SPECIES AND MORPHOLOGICAL ASSEMBLAGE VARIABILITY}

Sponge species assemblage and abundance data were highly correlated with the morphological assemblage data set. This means that it is possible to use morphological monitoring as a surrogate to predict spatial and temporal 
changes in the species assemblage. It is less time consuming to carry out rapid morphological monitoring (counting ten morpho types rather than trying to identify over 50 species) and this will be a more practical survey method for large areas of hard substratum, such as for habitat mapping and long-term monitoring schemes (Bell et al., 2006). Some small specimen collections will still have to be taken in order to ground truth the species present initially (Bell et al., 2006). It would be useful understand how the assemblages change through winter as this season was not sampled as a result of logistical and weather constraints in this study and there were clear shifts in morphological and species diversity between Autumn and Spring.

Both data sets varied significantly within and between sites and between seasons. The total number of sponge species/morphologies present at each site and season did not vary significantly. The seasonal patterns showed that the assemblages have the ability to return to a previous assemblage composition over an annual cycle, which supports previous work by Bell et al., (2006) using morphological monitoring of sponges over ten years at Skomer.

\subsubsection{THE CORRELATIONS OF ENVIRONMENTAL FACTORS WITH SKOMER MNR SPONGE ASSEMBLAGE VARIABILITY}

Physical factors are thought to be responsible for sponge species distribution over large temporal scales (Bell \& Barnes, 2000a; Carballo et al., 2008). Weather encompasses many of the physical factors that directly affect sessile species and assemblages in the marine environment. The factors that correlated with the change in species assemblage and morphological assemblage changes at Skomer were the combination of water temperature, maximum air net radiation per month and maximum wind gust speed or all of the above swapping the mean water temperature for mean air temperature. The shifts in morphological assemblage structure also correlated with the combination of Sechi disc readings and maximum air temperature. Temperature is one of the most important physical factors controlling the distribution of all organisms so it is not surprising that it is an important factor explaining the shifts in the sponge assemblage structure. Prior to the beginning of the survey period there 
was a hot summer and this may have triggered a change in assemblage structure before the surveying started. The responses of the assemblages were dynamic reflecting the seasonal and annual variation in environmental factors and other stochastic events. The abundance of sponges in Skomer MNR tends to decrease in summer. This could be a result of fusion of patches, however, although this did occur across all sites in particular with the encrusting sponges the majority of loses were due to patches disappearing completely. Sponge species are variable in their response to seasonal variability as some work has shown no link (Ayling, 1983b; Pansini \& Pronzato, 1985), while other work has shown more growth in summer (Elvin, 1976; Turon et al., 1998; Tanaka, 2002) or in winter (Orton, 1914; Johnson, 1979). It is also possible for cryptic species to have different patterns of growth and regression (Blanquer et al., 2008). Sponge species also vary in their larval release and settlement either releasing in summer, winter or all year round (Bergquist \& Sinclair, 1973).

Wind, which would influence sediment deposition, has been shown to play an important role in structuring sponge assemblages seasonally and annually in tropical environments (Carballo et al., 2008). Although many of the sponge species have adaptations to survive in the sedimented environment of Skomer MNR, adaptability will vary between species.

\subsubsection{MONITORING RECOMMENDATIONS}

In areas where species diversity is high it is recommended that an initial intensive species survey is carried out to identify the distribution patterns of the sponge species present and identify cryptic species. The collection of species-level data is approximately forty times more expensive than collecting morphological data due to extended in water time, laboratory time and the need for specialist taxonomic skills (Bell et al., 2006). However, species level surveys are important in order to identify species at the edge of their range, that are endemic, rare or those that are potentially economically valuable for biological compounds. Morphological data should be collected simultaneously in order to see if the patterns in morphology correlate with the species assemblage shifts. For some habitats this is not the case, for example, in caves and under boulders 
(Bell \& Barnes, 2001). The seasonal variability across all three sites shows that it is important to survey the sponge assemblages at the same time each year, if resources only allow one sampling interval each year, so that if there are any changes in assemblage composition (e.g. in response to anthropogenic impacts) this will be clearer against natural seasonal variation. Summer is recommended for better weather conditions in temperate climates and therefore a higher probability of being able to repeat the survey at the same time each year. Species-level surveys could be repeated at longer intervals or if there has been a sudden change in the morphological assemblage structure which is outside of the normal levels of variability for the area. Skomer has now been morphologically monitored annually since 1993 (Bell et al., 2006). During that time period there was distinctive shift in assemblage structure in 1995 from Bells work and 2009 from this study across all sites monitored that suggests annual morphological monitoring should highlight a significant shift which was also obvious in the species level monitoring. As the timelines on databases of sponge data increase, the longer term patterns start to emerge which is essential for planning future monitoring programmes and utilising money, time and expertise effectively as budgets for monitoring become tighter. 
5.6 APPENDIX OF THE SIMPER SIMILARITY PERCENTAGES - TWO-WAY ANALYSIS OF SPECIES CONTRIBUTIONS OF SITE CROSSED WITH SEASON (60\% CONTRIBUTION CUT OFF).

Table 5.7 Species explaining the similarities within Broad Gulley across all seasons. Average percentage similarity between all quadrats across all seasons $64.22 \%$

\begin{tabular}{|l|c|c|c|c|c|}
\hline Species & Av.Abund & Av.Sim & Sim/SD & Contrib. \% & Cum. \% \\
\hline Polymastia boletiformis & 3.56 & 10.32 & 5.05 & 16.07 & 16.07 \\
Stelligera stuposa & 2.76 & 7.02 & 3.69 & 10.93 & 26.99 \\
Raspailia/Stelligera juveniles & 2.71 & 6.48 & 3.29 & 10.09 & 37.08 \\
Cliona celata & 2.18 & 5.89 & 4.09 & 9.18 & 46.26 \\
Polymastia mamillaris & 2.04 & 4.62 & 2.01 & 7.20 & 53.46 \\
Suberites carnosus & 1.87 & 4.06 & 1.41 & 6.32 & 59.78 \\
Axinella damicornis & 1.34 & 2.68 & 1.33 & 4.18 & 63.96 \\
\hline
\end{tabular}

Table 5.8 Species explaining the similarities within Dog Leg across all seasons. Average percentage similarity between all quadrats across all seasons $73.59 \%$.

\begin{tabular}{|l|c|c|c|c|c|}
\hline Species & Av.Abund & Av.Sim & Sim/SD & Contrib. \% & Cum. \% \\
\hline Polymastia boletiformis & 3.41 & 6.62 & 8.73 & 9.00 & 9.00 \\
Raspailia/Stelligera juveniles & 3.56 & 6.62 & 8.64 & 9.00 & 18.00 \\
Stelligera stuposa & 3.38 & 6.24 & 5.20 & 8.47 & 26.47 \\
Hymeraphia stellifera & 3.63 & 6.09 & 2.97 & 8.27 & 34.75 \\
Suberites carnosus & 2.64 & 4.97 & 3.36 & 6.76 & 41.50 \\
Halicnemia patera & 2.59 & 3.78 & 1.75 & 5.13 & 46.64 \\
Polymastia mamillaris & 2.10 & 3.49 & 1.80 & 4.75 & 51.39 \\
Cliona celata & 1.93 & 3.34 & 3.50 & 4.53 & 55.92 \\
Homaxinella subdola & 1.91 & 3.31 & 1.58 & 4.50 & 60.42 \\
\hline
\end{tabular}

Table 5.9 Species explaining the similarities within Spongy Hillocks across all seasons. Average percentage similarity between all quadrats across all seasons $64.19 \%$.

\begin{tabular}{|l|c|c|c|c|c|}
\hline Species & Av.Abund & Av.Sim & Sim/SD & Contrib. \% & Cum. \% \\
\hline Stelligera stuposa & 3.28 & 7.09 & 6.51 & 11.04 & 11.04 \\
Polymastia boletiformis & 3.17 & 6.85 & 4.62 & 10.67 & 21.71 \\
Raspailia/Stelligera juveniles & 3.07 & 6.21 & 3.13 & 9.67 & 31.38 \\
Suberites carnosus & 2.51 & 4.85 & 2.26 & 7.56 & 38.95 \\
Hymeraphia stellifera & 2.49 & 4.03 & 1.58 & 6.28 & 45.23 \\
Halicnemia patera & 2.03 & 3.28 & 1.49 & 5.11 & 50.34 \\
Plocamionida ambigua / & 1.88 & 3.19 & 2.29 & 4.96 & 55.30 \\
Antho sp. & & & & & \\
Phorbas dives & 1.79 & 3.16 & 1.79 & 4.92 & 60.22 \\
\hline
\end{tabular}


Table 5.10 Species explaining the differences between Broad Gulley and Dogs Leg across all seasons. Average percentage dissimilarity between the sites across all seasons $=37.28 \%$

\begin{tabular}{|l|c|c|c|c|c|c|}
\hline Species & $\begin{array}{c}\text { BG Av. } \\
\text { Abund }\end{array}$ & $\begin{array}{c}\text { DL Av. } \\
\text { Abund }\end{array}$ & $\begin{array}{c}\text { Av. } \\
\text { Diss }\end{array}$ & $\begin{array}{c}\text { Diss/ } \\
\text { SD }\end{array}$ & $\begin{array}{c}\text { Contrib } \\
\%\end{array}$ & $\begin{array}{c}\text { Cum. } \\
\%\end{array}$ \\
\hline Hymeraphia stellifera & 1.59 & 3.63 & 2.70 & 1.56 & 7.23 & 7.23 \\
Halicnemia patera & 1.17 & 2.59 & 1.97 & 1.44 & 5.29 & 12.52 \\
Homaxinella subdola & 1.25 & 1.91 & 1.57 & 1.33 & 4.22 & 16.74 \\
Axinella dissimilis & 0.91 & 2.05 & 1.55 & 1.51 & 4.15 & 20.88 \\
Phorbas dives & 0.88 & 1.66 & 1.39 & 1.35 & 3.74 & 24.63 \\
Plocamionida ambigua / Antho sp. & 1.34 & 1.97 & 1.36 & 1.34 & 3.64 & 28.27 \\
Haliclona (Haliclona) oculata & 0.73 & 1.20 & 1.29 & 1.09 & 3.46 & 31.73 \\
Raspailia/Stelligera juveniles & 2.71 & 3.56 & 1.28 & 1.24 & 3.44 & 35.17 \\
Suberites carnosus & 1.87 & 2.64 & 1.27 & 1.26 & 3.42 & 38.59 \\
Stelligera rigida & 0.83 & 1.58 & 1.21 & 1.50 & 3.25 & 41.85 \\
Tethyspira spinosa & 0.27 & 1.11 & 1.15 & 1.57 & 3.08 & 44.92 \\
Stelligera stuposa & 2.76 & 3.38 & 1.12 & 1.33 & 3.00 & 47.92 \\
Polymastia mamillaris & 2.04 & 2.10 & 1.10 & 1.18 & 2.95 & 50.87 \\
Polymastia other sp & 1.16 & 1.50 & 1.07 & 1.28 & 2.87 & 53.74 \\
Red Encrusting & 1.04 & 1.49 & 1.05 & 1.24 & 2.81 & 56.56 \\
Raspailia (Raspailia) ramosa & 0.49 & 1.10 & 1.05 & 1.31 & 2.81 & 59.37 \\
Haliclona (Haliclona) urceolus & 0.97 & 1.04 & 1.00 & 1.22 & 2.70 & 62.06 \\
\hline
\end{tabular}

Table 5.11 Species explaining the differences between Broad Gulley and Spongy Hillocks across all seasons. Average percentage dissimilarity between the sites across all seasons $=39.62 \%$

\begin{tabular}{|l|c|c|c|c|c|c|}
\hline Species & $\begin{array}{c}\text { BG Av. } \\
\text { Abund }\end{array}$ & $\begin{array}{c}\text { SH Av. } \\
\text { Abund }\end{array}$ & $\begin{array}{c}\text { Av. } \\
\text { Diss }\end{array}$ & $\begin{array}{c}\text { Diss/ } \\
\text { SD }\end{array}$ & $\begin{array}{c}\text { Contrib } \\
\%\end{array}$ & $\begin{array}{c}\text { Cum. } \\
\%\end{array}$ \\
\hline Hymeraphia stellifera & 1.59 & 2.49 & 2.07 & 1.31 & 5.23 & 5.23 \\
Halicnemia patera & 1.17 & 2.03 & 1.81 & 1.34 & 4.58 & 9.81 \\
Phorbas dives & 0.88 & 1.79 & 1.62 & 1.42 & 4.10 & 13.90 \\
Homaxinella subdola & 1.25 & 1.78 & 1.60 & 1.32 & 4.04 & 17.94 \\
Cliona celata & 2.18 & 1.24 & 1.45 & 1.27 & 3.65 & 21.59 \\
Amphilectus fucorum & 0.71 & 1.23 & 1.41 & 1.22 & 3.57 & 25.16 \\
Suberites carnosus & 1.87 & 2.51 & 1.40 & 1.30 & 3.54 & 28.70 \\
Raspailia/Stelligera juveniles & 2.71 & 3.07 & 1.35 & 1.22 & 3.40 & 32.10 \\
Plocamionida ambigua / Antho sp. & 1.34 & 1.88 & 1.34 & 1.29 & 3.39 & 35.49 \\
Haliclona (Haliclona) oculata & 0.73 & 1.11 & 1.28 & 1.13 & 3.23 & 38.72 \\
Haliclona (Haliclona) urceolus & 0.97 & 1.24 & 1.27 & 1.19 & 3.20 & 41.92 \\
Polymastia mamillaris & 2.04 & 1.45 & 1.24 & 1.34 & 3.12 & 45.04 \\
Axinella dissimilis & 0.91 & 1.09 & 1.23 & 1.39 & 3.10 & 48.14 \\
Red Encrusting & 1.04 & 1.15 & 1.20 & 1.23 & 3.03 & 51.18 \\
Stelligera stuposa & 2.76 & 3.28 & 1.11 & 1.27 & 2.81 & 53.99 \\
Polymastia other sp. & 1.16 & 0.65 & 1.10 & 1.39 & 2.79 & 56.77 \\
Other Encrusting & 0.57 & 0.44 & 1.03 & 0.91 & 2.61 & 59.38 \\
Stelligera rigida & 0.83 & 1.08 & 1.01 & 1.26 & 2.56 & 61.93 \\
\hline
\end{tabular}


Table 5.12 Species explaining the differences between Dogs Leg and Spongy Hillocks across all seasons. Average percentage dissimilarity between the sites across all seasons $34.00 \%$

\begin{tabular}{|l|c|c|c|c|c|c|}
\hline Species & $\begin{array}{c}\text { DL Av. } \\
\text { Abund }\end{array}$ & $\begin{array}{c}\text { SH Av. } \\
\text { Abund }\end{array}$ & $\begin{array}{c}\text { Av. } \\
\text { Diss }\end{array}$ & $\begin{array}{c}\text { Diss/ } \\
\text { SD }\end{array}$ & $\begin{array}{c}\text { Contrib } \\
\%\end{array}$ & $\begin{array}{c}\text { Cum. } \\
\%\end{array}$ \\
\hline Hymeraphia stellifera & 3.63 & 2.49 & 1.68 & 1.37 & 4.95 & 4.95 \\
Halicnemia patera & 2.59 & 2.03 & 1.46 & 1.22 & 4.28 & 9.23 \\
Axinella dissimilis & 2.05 & 1.09 & 1.39 & 1.44 & 4.08 & 13.31 \\
Haliclona (Haliclona) oculata & 1.20 & 1.11 & 1.33 & 1.35 & 3.92 & 17.23 \\
Polymastia mamillaris & 2.10 & 1.45 & 1.21 & 1.44 & 3.55 & 20.78 \\
Amphilectus fucorum & 0.71 & 1.23 & 1.19 & 1.30 & 3.50 & 24.28 \\
Polymastia other sp. & 1.50 & 0.65 & 1.17 & 1.46 & 3.44 & 27.72 \\
Plocamionida ambigua / Antho sp. & 1.97 & 1.88 & 1.15 & 1.30 & 3.39 & 31.11 \\
Homaxinella subdola & 1.91 & 1.78 & 1.13 & 1.19 & 3.32 & 34.43 \\
Cliona celata & 1.93 & 1.24 & 1.06 & 1.32 & 3.11 & 37.54 \\
Phorbas dives & 1.66 & 1.79 & 1.03 & 1.22 & 3.02 & 40.57 \\
Red Encrusting & 1.49 & 1.15 & 1.02 & 1.35 & 3.01 & 43.58 \\
Haliclona (Haliclona) urceolus & 1.04 & 1.24 & 1.00 & 1.16 & 2.95 & 46.53 \\
Raspailia/Stelligera juveniles & 3.56 & 3.07 & 0.94 & 1.24 & 2.76 & 49.30 \\
Halichondria sp. (yellow) & 0.70 & 0.68 & 0.92 & 1.10 & 2.72 & 52.01 \\
Stelligera rigida & 1.58 & 1.08 & 0.90 & 1.39 & 2.66 & 54.67 \\
Tethyspira spinosa & 1.11 & 0.71 & 0.86 & 1.35 & 2.53 & 57.21 \\
Clathria (Microciona) armata & 0.86 & 0.39 & 0.86 & 1.03 & 2.52 & 59.73 \\
Antho (Antho) inconstans & 0.85 & 0.55 & 0.85 & 1.15 & 2.51 & 62.24 \\
\hline
\end{tabular}

Table 5.13 Species explaining the similarities within spring across all sites. Average similarity within the spring season across all sites $=69.27 \%$

\begin{tabular}{|l|c|c|c|c|c|}
\hline Species & Av.Abund & Av.Sim & Sim/SD & Contrib\% & Cum.\% \\
\hline Polymastia boletiformis & 3.47 & 7.69 & 4.62 & 11.10 & 11.10 \\
Stelligera stuposa & 3.34 & 7.05 & 6.59 & 10.17 & 21.28 \\
Hymeraphia stellifera & 3.28 & 6.02 & 2.35 & 8.69 & 29.97 \\
Suberites carnosus & 2.62 & 5.45 & 4.78 & 7.87 & 37.84 \\
Raspailia/Stelligera juveniles & 2.82 & 5.26 & 2.47 & 7.59 & 45.43 \\
Polymastia mamillaris & 2.26 & 4.11 & 2.07 & 5.93 & 51.36 \\
Halicnemia patera & 2.15 & 3.59 & 1.66 & 5.18 & 56.54 \\
Haliclona (Haliclona) urceolus & 2.01 & 3.42 & 1.60 & 4.94 & 61.48 \\
\hline
\end{tabular}


Table 5.14 Species explaining the similarities within summer across all sites. Average similarity within the summer season across all sites $=65.64 \%$

\begin{tabular}{|l|c|c|c|c|c|}
\hline Species & Av.Abun & Av.Sim & Sim/SD & Contrib\% & Cum.\% \\
\hline Polymastia boletiformis & 3.25 & 7.71 & 3.32 & 11.75 & 11.75 \\
Stelligera stuposa & 3.18 & 7.08 & 5.29 & 10.79 & 22.53 \\
Raspailia/Stelligera juveniles & 3.05 & 6.67 & 4.72 & 10.17 & 32.70 \\
Suberites carnosus & 2.26 & 4.38 & 1.76 & 6.67 & 39.38 \\
Cliona celata & 1.75 & 3.39 & 1.37 & 5.16 & 44.53 \\
Hymeraphia stellifera & 2.07 & 3.30 & 1.35 & 5.03 & 49.57 \\
Homaxinella subdola & 1.79 & 3.20 & 1.56 & 4.87 & 54.44 \\
Polymastia mamillaris & 1.67 & 3.04 & 1.52 & 4.63 & 59.07 \\
Plocamionida ambigua / Antho sp. & 1.66 & 2.90 & 1.90 & 4.41 & 63.48 \\
\hline
\end{tabular}

Table 5.15 Species explaining the similarities within autumn across all sites. Average similarity within the autumn season across all sites $=65.36 \%$

\begin{tabular}{|l|c|c|c|c|c|}
\hline Species & Av.Abund & Av.Sim & Sim/SD & Contrib\% & Cum.\% \\
\hline Polymastia boletiformis & 3.41 & 7.88 & 3.15 & 12.05 & 12.05 \\
Raspailia/Stelligera juveniles & 3.53 & 7.20 & 6.02 & 11.01 & 23.06 \\
Stelligera stuposa & 2.88 & 5.92 & 3.43 & 9.06 & 32.12 \\
Suberites carnosus & 2.20 & 4.35 & 1.72 & 6.66 & 38.77 \\
Cliona celata & 1.90 & 3.73 & 2.01 & 5.70 & 44.48 \\
Stelligera rigida & 1.71 & 3.32 & 2.29 & 5.08 & 49.56 \\
Hymeraphia stellifera & 2.49 & 3.08 & 1.18 & 4.71 & 54.27 \\
Axinella damicornis & 1.57 & 2.91 & 1.93 & 4.45 & 58.72 \\
Polymastia mamillaris & 1.56 & 2.80 & 1.21 & 4.28 & 62.99 \\
\hline
\end{tabular}

Table 5.16 Species explaining the differences between spring and summer across all sites. Average percentage dissimilarity between spring and summer across all sites $=34.19 \%$

\begin{tabular}{|l|c|c|c|c|c|c|}
\hline Species & $\begin{array}{c}\text { Spr. Av. } \\
\text { Abund }\end{array}$ & $\begin{array}{c}\text { Sum. Av. } \\
\text { Abund }\end{array}$ & $\begin{array}{c}\text { Av. } \\
\text { Diss }\end{array}$ & $\begin{array}{c}\text { Diss/ } \\
\text { SD }\end{array}$ & $\begin{array}{c}\text { Contrib } \\
\%\end{array}$ & $\begin{array}{c}\text { Cum. } \\
\%\end{array}$ \\
\hline Hymeraphia stellifera & 3.28 & 2.07 & 1.86 & 1.37 & 5.43 & 5.43 \\
Haliclona (Haliclona) urceolus & 2.01 & 0.92 & 1.71 & 1.30 & 5.01 & 10.44 \\
Halicnemia patera & 2.15 & 1.72 & 1.37 & 1.30 & 4.01 & 14.45 \\
Homaxinella subdola & 1.60 & 1.79 & 1.31 & 1.25 & 3.84 & 18.29 \\
Haliclona (Haliclona) oculata & 0.58 & 1.06 & 1.24 & 1.29 & 3.63 & 21.92 \\
Amphilectus fucorum & 1.03 & 0.99 & 1.20 & 1.31 & 3.50 & 25.42 \\
Polymastia mamillaris & 2.26 & 1.67 & 1.19 & 1.23 & 3.47 & 28.89 \\
Plocamionida ambigua /Antho sp. & 1.91 & 1.66 & 1.16 & 1.36 & 3.39 & 32.29 \\
Axinella dissimilis & 1.57 & 1.32 & 1.16 & 1.32 & 3.39 & 35.68 \\
Raspailia/Stelligera juveniles & 2.82 & 3.05 & 1.14 & 1.10 & 3.35 & 39.03 \\
Red Encrusting & 1.26 & 1.02 & 1.04 & 1.26 & 3.05 & 42.08 \\
Phorbas dives & 1.60 & 1.31 & 1.03 & 1.25 & 3.01 & 45.09 \\
Suberites carnosus & 2.62 & 2.26 & 1.00 & 1.12 & 2.91 & 48.00 \\
Stelligera rigida & 0.73 & 1.11 & 0.95 & 1.27 & 2.79 & 50.79 \\
Cliona celata & 1.57 & 1.75 & 0.89 & 1.17 & 2.60 & 53.39 \\
Raspailia (Raspailia) ramosa & 0.90 & 0.74 & 0.84 & 1.26 & 2.44 & 55.83 \\
Axinella damicornis & 1.20 & 1.39 & 0.83 & 1.15 & 2.42 & 58.25 \\
Other Encrusting & 0.22 & 0.53 & 0.83 & 0.81 & 2.42 & 60.67 \\
\hline
\end{tabular}


Table 5.17 Species explaining the differences between spring and autumn across all sites. Average percentage dissimilarity between spring and summer across all sites $=36.52 \%$

\begin{tabular}{|l|c|c|c|c|c|c|}
\hline Species & $\begin{array}{c}\text { Spr Av. } \\
\text { Abund }\end{array}$ & $\begin{array}{c}\text { Aut Av. } \\
\text { Abund }\end{array}$ & $\begin{array}{c}\text { Av. } \\
\text { Diss }\end{array}$ & $\begin{array}{c}\text { Diss/ } \\
\text { SD }\end{array}$ & $\begin{array}{c}\text { Contrib } \\
\%\end{array}$ & $\begin{array}{c}\text { Cum. } \\
\%\end{array}$ \\
\hline Haliclona (Haliclona) urceolus & 2.01 & 0.32 & 2.12 & 1.54 & 5.82 & 5.82 \\
Hymeraphia stellifera & 3.28 & 2.49 & 2.01 & 1.28 & 5.50 & 11.32 \\
Haliclona (Haliclona) oculata & 0.58 & 1.47 & 1.66 & 1.23 & 4.54 & 15.85 \\
Halicnemia patera & 2.15 & 2.03 & 1.65 & 1.39 & 4.53 & 20.38 \\
Homaxinella subdola & 1.60 & 1.50 & 1.36 & 1.26 & 3.72 & 24.10 \\
Plocamionida ambigua / Antho sp & 1.91 & 1.68 & 1.30 & 1.30 & 3.57 & 27.67 \\
Stelligera rigida & 0.73 & 1.71 & 1.30 & 1.60 & 3.56 & 31.23 \\
Axinella dissimilis & 1.57 & 1.05 & 1.25 & 1.37 & 3.44 & 34.67 \\
Polymastia mamillaris & 2.26 & 1.56 & 1.23 & 1.25 & 3.36 & 38.03 \\
Raspailia/Stelligera juveniles & 2.82 & 3.53 & 1.19 & 1.12 & 3.25 & 41.29 \\
Phorbas dives & 1.60 & 1.58 & 1.16 & 1.29 & 3.17 & 44.45 \\
Amphilectus fucorum & 1.03 & 0.65 & 1.14 & 1.14 & 3.11 & 47.57 \\
Polymastia other sp & 1.19 & 1.03 & 1.05 & 1.20 & 2.88 & 50.45 \\
Antho (Antho) inconstans & 0.39 & 0.90 & 0.92 & 1.05 & 2.53 & 52.98 \\
Red Encrusting & 1.26 & 1.48 & 0.92 & 1.25 & 2.51 & 55.49 \\
Stelligera stuposa & 3.34 & 2.88 & 0.91 & 1.20 & 2.48 & 57.97 \\
Paratimea constellata & 0.30 & 0.94 & 0.89 & 0.81 & 2.43 & 60.40 \\
\hline
\end{tabular}

Table 5.18 Species explaining the differences between autumn and summer across all sites. Average percentage dissimilarity between autumn and summer across all sites $=37.17 \%$

\begin{tabular}{|l|c|c|c|c|c|c|}
\hline Species & $\begin{array}{c}\text { Sum } \\
\text { Av. } \\
\text { Abund }\end{array}$ & $\begin{array}{c}\text { Aut Av. } \\
\text { Abund }\end{array}$ & $\begin{array}{c}\text { Av. } \\
\text { Diss }\end{array}$ & $\begin{array}{c}\text { Diss/ } \\
\text { SD }\end{array}$ & $\begin{array}{c}\text { Contrib } \\
\%\end{array}$ & $\begin{array}{c}\text { Cum. } \\
\%\end{array}$ \\
\hline Hymeraphia stellifera & 2.07 & 2.49 & 1.91 & 1.49 & 5.14 & 5.14 \\
Halicnemia patera & 1.72 & 2.03 & 1.83 & 1.58 & 4.91 & 10.06 \\
Haliclona (Haliclona) oculata & 1.06 & 1.47 & 1.50 & 1.24 & 4.02 & 14.08 \\
Homaxinella subdola & 1.79 & 1.50 & 1.37 & 1.24 & 3.67 & 17.76 \\
Plocamionida ambigua /Antho sp. & 1.66 & 1.68 & 1.35 & 1.31 & 3.63 & 21.38 \\
Phorbas dives & 1.31 & 1.58 & 1.26 & 1.23 & 3.39 & 24.77 \\
Red Encrusting & 1.02 & 1.48 & 1.23 & 1.31 & 3.32 & 28.09 \\
Amphilectus fucorum & 0.99 & 0.65 & 1.22 & 1.13 & 3.27 & 31.36 \\
Raspailia/Stelligera juveniles & 3.05 & 3.53 & 1.18 & 1.44 & 3.17 & 34.53 \\
Suberites carnosus & 2.26 & 2.20 & 1.17 & 1.17 & 3.14 & 37.67 \\
Axinella dissimilis & 1.32 & 1.05 & 1.14 & 1.37 & 3.08 & 40.74 \\
Polymastia other sp. & 0.99 & 1.03 & 1.10 & 1.23 & 2.97 & 43.71 \\
Other Encrusting & 0.53 & 0.69 & 1.10 & 1.00 & 2.95 & 46.66 \\
Haliclona (Haliclona) urceolus & 0.92 & 0.32 & 1.09 & 1.16 & 2.93 & 49.60 \\
Paratimea constellata & 0.22 & 0.94 & 1.07 & 0.84 & 2.87 & 52.47 \\
Polymastia mamillaris & 1.67 & 1.56 & 1.05 & 1.17 & 2.84 & 55.31 \\
Clathria (Microciona) armata & 0.49 & 0.90 & 1.05 & 1.12 & 2.81 & 58.12 \\
Stelligera rigida & 1.11 & 1.71 & 1.01 & 1.35 & 2.73 & 60.85 \\
\hline
\end{tabular}


Table 5.19 Morphologies explaining the similarities within Spongy Hillocks across all seasons. Average percentage similarity between all quadrats across all seasons $78.79 \%$

\begin{tabular}{|l|c|c|c|c|l|}
\hline Morphology & Av.Abund & Av.Sim & Sim/SD & Contrib\% & Cum.\% \\
\hline Arborescent & 8.70 & 23.19 & 5.50 & 29.43 & 29.43 \\
Encrusting & 7.48 & 17.33 & 3.03 & 21.99 & 51.42 \\
Papillate & 5.48 & 14.28 & 5.93 & 18.12 & 69.54 \\
\hline
\end{tabular}

Table 5.20 Morphologies explaining the similarities within Broad Gulley across all seasons. Average percentage similarity between all quadrats across all seasons $77.45 \%$

\begin{tabular}{|l|c|c|c|c|l|}
\hline Morphology & Av.Abund & Av.Sim & Sim/SD & Contrib\% & Cum.\% \\
\hline Papillate & 6.83 & 21.62 & 7.60 & 27.92 & 27.92 \\
Arborescent & 6.89 & 18.89 & 4.56 & 24.39 & 52.31 \\
Encrusting & 4.99 & 10.82 & 2.28 & 13.97 & 66.28 \\
\hline
\end{tabular}

Table 5.21 Morphologies explaining the similarities within Dogs Leg across all seasons. Average percentage similarity between all quadrats across all seasons $84.33 \%$

\begin{tabular}{|l|c|c|c|c|l|}
\hline Morphology & Av.Abund & Av.Sim & Sim/SD & Contrib\% & Cum.\% \\
\hline Arborescent & 10.29 & 23.68 & 8.36 & 28.09 & 28.09 \\
Encrusting & 10.18 & 19.64 & 3.07 & 23.29 & 51.38 \\
Papillate & 6.70 & 15.46 & 9.06 & 18.34 & 69.71 \\
\hline
\end{tabular}

Table 5.22 Morphologies explaining the differences between Spongy Hillocks and Broad Gulley across all seasons. Average percentage dissimilarity between Spongy Hillocks and Broad Gulley across all seasons $25.95 \%$

\begin{tabular}{|l|c|c|c|c|c|l|}
\hline Morphology & SH Av.Abund & BG Av.Abund & Av.Diss & Diss/SD & Contrib\% & Cum.\% \\
\hline Encrusting & 7.48 & 4.99 & 6.00 & 1.29 & 23.14 & 23.14 \\
Arborescent & 8.70 & 6.89 & 4.36 & 1.30 & 16.82 & 39.96 \\
Pedunculate & 3.61 & 2.61 & 2.67 & 1.41 & 10.31 & 50.26 \\
Burrowing & 1.24 & 2.51 & 2.58 & 1.31 & 9.95 & 60.22 \\
\hline
\end{tabular}

Table 5.23 Morphologies explaining the differences between Spongy Hillocks and Dogs Leg across all seasons. Average percentage dissimilarity between Spongy Hillocks and Dogs Leg across all seasons $20.77 \%$

\begin{tabular}{|l|c|c|c|c|c|l|}
\hline Morphology & SH Av.Abund & DL Av.Abund & Av.Diss & Diss/SD & Contrib\% & Cum.\% \\
\hline Encrusting & 7.48 & 10.18 & 5.46 & 1.26 & 26.27 & 26.27 \\
Arborescent & 8.70 & 10.29 & 2.90 & 1.41 & 13.96 & 40.23 \\
Papillate & 5.48 & 6.70 & 2.10 & 1.50 & 10.09 & 50.32 \\
Burrowing & 1.24 & 2.28 & 1.91 & 1.42 & 9.21 & 59.53 \\
Pedunculate & 3.61 & 3.77 & 1.88 & 1.21 & 9.06 & 68.59 \\
\hline
\end{tabular}


Table 5.24 Morphologies explaining the differences between Broad Gulley and Dogs Leg across all seasons. Average percentage dissimilarity between Broad Gulley and Dogs Leg across all seasons $24.57 \%$

\begin{tabular}{|l|c|c|c|c|c|l|}
\hline Morphology & BG Av.Abund & DL Av.Abund & Av.Diss & Diss/SD & Contrib\% & Cum.\% \\
\hline Encrusting & 4.99 & 10.18 & 7.73 & 1.48 & 31.44 & 31.44 \\
Arborescent & 6.89 & 10.29 & 5.37 & 1.53 & 21.86 & 53.30 \\
Pedunculate & 2.61 & 3.77 & 2.48 & 1.33 & 10.08 & 63.38 \\
\hline
\end{tabular}

Table 5.25 Morphologies explaining the similarities within spring across all sites. Average percentage similarity within spring across all sites $81.53 \%$

\begin{tabular}{|l|c|c|c|c|c|}
\hline Morphology & Av.Abund & Av.Sim & Sim/SD & Contrib\% & Cum.\% \\
\hline Arborescent & 8.40 & 20.06 & 5.27 & 24.61 & 24.61 \\
Encrusting & 8.54 & 18.31 & 2.91 & 22.46 & 47.07 \\
Papillate & 6.86 & 16.65 & 4.08 & 20.42 & 67.49 \\
\hline
\end{tabular}

Table 5.26 Morphologies explaining the similarities within summer across all sites. Average percentage similarity within summer across all sites $79.57 \%$

\begin{tabular}{|l|c|c|c|c|c|}
\hline Morphology & Av.Abund & Av.Sim & Sim/SD & Contrib\% & Cum.\% \\
\hline Arborescent & 8.43 & 22.92 & 5.13 & 28.81 & 28.81 \\
Papillate & 5.86 & 16.39 & 4.17 & 20.60 & 49.41 \\
Encrusting & 6.31 & 15.51 & 2.79 & 19.49 & 68.89 \\
\hline
\end{tabular}

Table 5.27 Morphologies explaining the similarities within autumn across all sites. Average percentage similarity within autumn across all sites $78.09 \%$

\begin{tabular}{|l|c|c|c|c|c|}
\hline Morphology & Av.Abund & Av.Sim & Sim/SD & Contrib\% & Cum.\% \\
\hline Arborescent & 9.18 & 22.92 & 5.91 & 29.34 & 29.34 \\
Papillate & 6.20 & 17.03 & 4.62 & 21.80 & 51.14 \\
Encrusting & 8.33 & 14.81 & 1.70 & 18.97 & 70.11 \\
\hline
\end{tabular}

Table 5.28 Morphologies explaining the differences between spring and summer across all sites. Average percentage dissimilarity $21.30 \%$

\begin{tabular}{|l|c|c|c|c|c|c|}
\hline Morphology & Spr Av.Abund & Sum Av.Abund & Av.Diss & Diss/SD & Contrib\% & Cum.\% \\
\hline Encrusting & 8.54 & 6.31 & 4.78 & 1.41 & 22.45 & 22.45 \\
Tubular & 2.92 & 1.20 & 3.17 & 1.06 & 14.86 & 37.31 \\
Arborescent & 8.40 & 8.43 & 2.82 & 1.24 & 13.22 & 50.53 \\
Papillate & 6.86 & 5.86 & 2.09 & 1.38 & 9.80 & 60.34 \\
\hline
\end{tabular}

Table 5.29 Morphologies explaining the differences between spring and autumn across all sites. Average percentage dissimilarity $22.69 \%$

\begin{tabular}{|l|c|c|c|c|c|c|}
\hline Morphology & Spr Av.Abund & Aut Av.Abund & Av.Diss & Diss/SD & Contrib\% & Cum.\% \\
\hline Encrusting & 8.54 & 8.33 & 6.20 & 1.65 & 27.34 & 27.34 \\
\hline Tubular & 2.92 & 0.42 & 3.71 & 1.22 & 16.34 & 43.67 \\
\hline Arborescent & 8.40 & 9.18 & 2.96 & 1.24 & 13.03 & 56.70 \\
\hline Papillate & 6.86 & 6.20 & 1.97 & 1.29 & 8.69 & 65.39 \\
\hline
\end{tabular}


Chapter 5 Spatial and temporal variability of sponge species and morphological diversity

Table 5.30 Morphologies explaining the differences between autumn and summer across all sites. Average percentage dissimilarity $22.62 \%$

\begin{tabular}{|l|c|c|c|c|c|l|}
\hline Morphology & $\begin{array}{c}\text { Summer } \\
\text { Av.Abund }\end{array}$ & $\begin{array}{c}\text { Autumn } \\
\text { Av.Abund }\end{array}$ & Av.Diss & Diss/SD & Contrib\% & Cum.\% \\
\hline Encrusting & 6.31 & 8.33 & 6.97 & 1.71 & 30.80 & 30.80 \\
\hline Arborescent & 8.43 & 9.18 & 3.42 & 1.31 & 15.10 & 45.89 \\
\hline Stalked & 3.19 & 3.17 & 2.25 & 1.33 & 9.95 & 55.85 \\
\hline Repent & 1.25 & 0.78 & 1.85 & 1.12 & 8.16 & 64.01 \\
\hline
\end{tabular}




\section{CHAPTER 6}

TEMPORAL AND SPATIAL DIVERSITY PATTERNS OF SPONGE ASSEMBLAGES IN THE WAKATOBI MARINE NATIONAL PARK, INDONESIA. 
Chapter 6 Temporal and spatial diversity patterns of sponge assemblages in the Wakatobi Marine National Park, Indonesia.

\subsection{ABSTRACT}

The preservation of biodiversity is vital for maintaining ecosystem function and stability, as well as sustaining the economic potential of species, habitats and assemblages. Knowing how the diversity is partitioned within an area can provide insights into patterns of biodiversity and their underlying processes. The Indo-Pacific is known to contain some of the most diverse marine assemblages in the world, including sponges, which have many important functional roles. This chapter investigates the temporal and spatial diversity of sponge assemblages on an Indonesian coral reef over a 5-year period. One hundred and thirty six sponge 0.T.U.'s (operational taxonomic units) across thirty families were recorded at three sites over a five-year period. The mean O.T.U. richness differed significantly among the sites (Pseudo $F=9.9263$ $\mathrm{P}=0.0003$ ), but not between the years. Partitioning diversity showed that for species richness the betaregion component was the most important factor explaining $50-60 \%$ of the variation and turnover of ephemeral species between years. There were significant changes in the assemblage composition and abundance at the quadrat and site scale, and between years. However, the partitioning of sponge assemblage diversity remained consistent between years for the Species richness, Shannon Index and Simpsons Index. The consistency of diversity partitioning means that although the abundances and presence of different O.T.U's did vary between years, the ratio of the diversity within the quadrats compared to the site and region remains consistent and therefore the annual variation of beta diversity appears to be low. This means that the actual species present and abundances were significantly different between sites and years, however, the proportion of species distributed within the quadrats and within the sites remained consistent between years even if the different species were present. 
Chapter 6 Temporal and spatial diversity patterns of sponge assemblages in the Wakatobi Marine National Park, Indonesia.

\subsection{INTRODUCTION}

The preservation of biodiversity is vital for maintaining ecosystem function and stability, as well as sustaining the economic potential of species, habitats and assemblages (Hooper et al., 2005; Worm et al., 2006; Costello et al., 2010). Assessment and monitoring in order to safeguard biodiversity has shifted focus from the management of individual species within habitats to the preservation of entire assemblages within ecoregions (Gaston et al., 2001; Pressey, 2004). This requires an understanding of the patterns of spatial and temporal turnover in assemblage composition within the region, as well an understanding of the mechanisms explaining these patterns (Summerville et al., 2003; Pressey, 2004; Rondinini et al., 2006; Grantham et al., 2010).

Sponges are found in aquatic habitats worldwide (Dayton, 1978; Alvarez et al., 1990; Diaz et al., 1993; Økland \& Økland, 1996; Bell \& Barnes, 2000a; Hooper et al., 2002; Bell \& Smith, 2004; Leys et al., 2004). The Indo-Pacific is known to contain some of the most diverse marine assemblages in the world, and Indonesia is at the centre of this biodiversity hotspot (Edinger et al., 2000; Roberts et al., 2002; Karlson et al., 2004). Both speciation and extinction rates are likely to be high in the Indo-Pacific, resulting in especially high endemism (high alpha diversity and beta diversity) (Reaka et al., 2008).

There have been some earlier studies of the ecology and spatial patterns of Indonesian sponge assemblages (Amir, 1992; de Voogd et al., 1999; Bell \& Smith, 2004; de Voogd et al., 2004; Cleary et al., 2005; Becking et al., 2006; de Voogd et al., 2006; Bell, 2007a, b; de Voogd \& Cleary, 2007, 2008; Bell et al., 2010; Powell et al., 2010). Spatial patterns of biodiversity have been linked to environmental gradients such as depth, sedimentation, and the degree of human settlement, however, there are no published works on the temporal variability of sponge assemblages in the Indo-Pacific region. Temporal variation in sponges from other areas has been reported at the species level (Dayton, 
Chapter 6 Temporal and spatial diversity patterns of sponge assemblages in the Wakatobi Marine National Park, Indonesia.

1989; Garrabou \& Zabala, 2001; Wulff, 2007; de Caralt et al., 2008; Koopmans \& Wijffels, 2008; McMurray et al., 2008). To date, there have been relatively few studies examining temporal variation in entire sponge assemblages over more than a year, but data is available from the Caribbean (Hughes, 1996; Wulff, 2006a), in the tropical Eastern Pacific (Carballo et al., 2008) and in temperate waters (see chapters 4 and 5 of this thesis) (Sara, 1970; Pansini \& Pronzato, 1990; Bell et al., 2006). Research from the Mediterranean has shown slow growth within sponge assemblages with no clear seasonality and some sponge tissue regression, while the abundance of different sponge species remained similar throughout an eight year study (Sara, 1970; Pansini \& Pronzato, 1990). Similar results showing little overall change have also been reported from sites in the Atlantic (Fowler \& Laffoley, 1993; Hiscock, 1994) and sites in the Caribbean (Hughes, 1996). In fact, even though the overall abundance within sponge assemblages remained relatively stable in the Caribbean sites, the actual individual species were very variable suggesting recruitment and mortality were in balance in these assemblages (Hughes, 1996). However, more recent work in the Atlantic, Caribbean and Pacific suggests that swift changes in sponge assemblages are common and recruitment and mortality do not always occur in equilibrium and therefore the overall assemblage composition can show dramatic changes (Bell et al., 2006; Wulff, 2006; Carballo et al., 2008).

In order to target conservation efforts appropriately it is important to identify the primary source of diversity (Veech et al., 2002). Total diversity (gamma) of an area can be partitioned into the components of mean diversity within samples (alpha) and diversity among samples (beta), which has spatial (beta site and betaregion) and temporal components (betaseasonal and betaannual) (Whittaker, 1960, 1972). Lande (1996) developed the concept of additive partitioning of diversity using the alpha, beta and gamma components described by Whittaker, although similar work using different names has been undertaken by MacArthur et al. (1966) and Allan (1975). Alpha and beta components of biodiversity decompose regional diversity into two parts: a measure of average single-location (or single-assemblage) diversity and a measure of the relative 
Chapter 6 Temporal and spatial diversity patterns of sponge assemblages in the Wakatobi Marine National Park, Indonesia.

change in species composition between locations (or assemblages) (Jost, 2007). One of the advantages of additive partitioning is that it allows the analysis of diversity found in a hierarchy of different scales (Veech \& Crist, 2010b, a). A variety of processes interact on multiple spatial scales in order to have a direct impact on the immigration and survival of organisms in an area (Lawton, 1999). Although some factors will have wide impacts across all spatial scales, the environmental variables explaining species diversity are different depending on the size and time scale over which species diversity is measured (Willis \& Whittaker, 2002). For example, at small spatial scales, local interactions such as spatial competition may be important for alpha diversity, while at larger spatial scales, dispersal limitation of temporal factors may be significant for beta diversity.

There are very few previous studies comparing temporal changes in the additive partitioning of diversity (Summerville \& Crist, 2005; Wu et al., 2010). The effect of season has been found to contribute a large proportion of the biodiversity in terrestrial invertebrate studies in temperate regions (Summerville \& Crist, 2005; Wu et al., 2010), however, temporal effects in the tropical and/or marine environment of partitioning diversity have not been previously investigated. In order to protect a habitat it is important to know if all the biodiversity is contained within a small area or across a large region and therefore what size area needs to be managed in order to protect the biodiversity within it (Zvuloni et al., 2010). Only one study has incorporated annual turnover into diversity partitioning and these authors also found that betaannual diversity was low for species richness and evenness for butterflies in a beech maple forest in Ohio (Summerville \& Crist, 2005).

The major component of the diversity of tropical butterflies within a region was found to be due to the differences between sampling months (temporal variation) rather than spatial variation due to varying sites surveyed (DeVries et al., 1997). Partitioning diversity is a powerful tool for quantifying spatial and temporal variation because it provides a single set of values of alpha and beta 
Chapter 6 Temporal and spatial diversity patterns of sponge assemblages in the Wakatobi Marine National Park, Indonesia.

diversity for a given sampling scale and therefore gives a simple, intuitive measure of species diversity and composition, in a way that is accessible to conservation managers, ecologists, and non-scientists (Veech \& Crist, 2010a). Knowing how the diversity is partitioned within an area can provide insights into the extent to which patterns of biodiversity (and their underlying processes) are likely to influence effective spatial boundaries (for example where to place a marine reserve effectively in order to protect an area which is significant for biodiversity) for conservation management (Semmens et al., 2010).

Additive partitioning has been mainly applied to terrestrial invertebrate diversity (DeVries et al., 1997; Gering et al., 2003; Summerville et al., 2003; Jost et al., 2009), birds (Fleishman et al., 2003; Kattan et al., 2006; Wu et al., 2010), and plants (Chandy et al., 2006; Chen et al., 2008; Klimek et al., 2008), however, recently there have been a few studies applying the technique to freshwater invertebrates (Stendera \& Johnson, 2005), coral reef fish (Belmaker et al., 2008) and coral reefs (Zvuloni et al., 2010). For many coral reef organisms, gamma diversity peaks around the Indo-Pacific triangle (Belmaker et al., 2008). Areas that are species rich often have multiple species that can perform similar functions (Walker, 1992). Species that perform similar functions can be said to be ecologically redundant, as the species are similar in function so that if one species is removed and replaced by another, no loss of ecosystem function would be observed (Lawton \& Brown, 1993). This is equivalent to "functional degeneracy" (functional redundancy) as recently described by Gonzalez \& Loreau (2009). This situation does not imply that each redundant species has redundant impacts rather that these redundant species provide the stability within an assemblage to adapt to varying environmental and physical conditions. Therefore in a specious area like the Indo-Pacific it is likely that there are many redundant species that would lead to the alpha, beta and gamma diversity remaining relatively stable in partitioning, while the actual species that make up the diversity will change in abundance to response to 
Chapter 6 Temporal and spatial diversity patterns of sponge assemblages in the Wakatobi Marine National Park, Indonesia.

environmental variability and other factors. This chapter will compare the patterns of sponge diversity spatially and temporally across local spatial scales within the same habitat type in the Indo Pacific.

AIMS

1. What is the species diversity and abundance of sponge assemblages in Wakatobi Marine National Park (WNMP)?

2. How is diversity partitioned over a range of spatial scales??

3. Does the diversity partitioning remain consistent between years? 
Chapter 6 Temporal and spatial diversity patterns of sponge assemblages in the Wakatobi Marine National Park, Indonesia.

\subsection{Methods}

\subsubsection{StUdy AREA}

The Wakatobi National Marine Park (WNMP), which covers 1.3 million hectares, is situated in the Tukang Besi archipelago, which is a remote island group off the coast of S.E. Sulawesi in Indonesia (3-6º S, $120^{\circ} 45^{\prime}-124^{\circ} 06^{\prime}$ E) (Fig. 6.1).

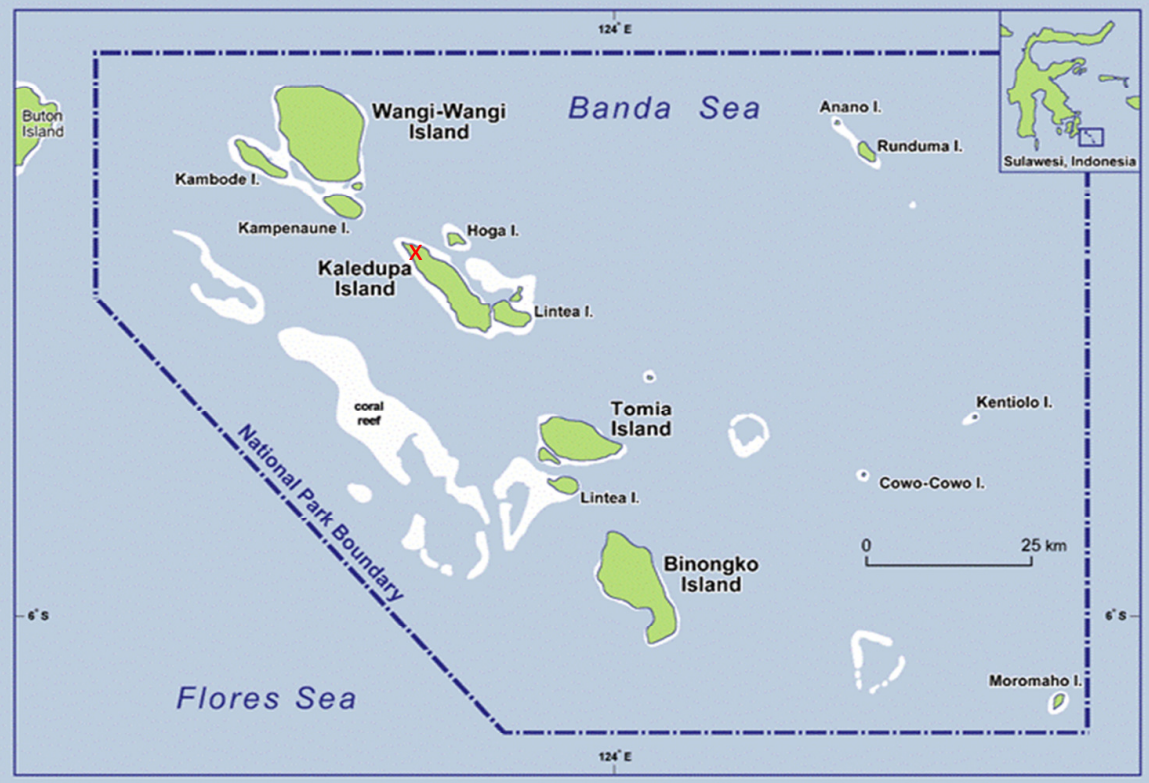

Wakatobi Marine National Park, South East Sulawesi

Fig. 6.1 Location of survey sites off Hoga Island, South-East Sulawesi (boxed top right) Wakatobi National Marine Park (WMNP) with the location of the survey site on the fringing coral reef off Hoga Island. The white shading indicates coral reef within the WMNP (map from Operation Wallacea).

A no-take zone was established in 2003 within the park, however, the funding has since lapsed and therefore there is currently no enforcement within the previously protected zone. The Hoga Island reef is characterised by relatively high light penetration $(10 \mathrm{~m} \pm 3$ using a secchi disc vertically next to the reef wall) and low sedimentation rates as the coarse sand particles settle quickly (7.54 \pm 0.76 g dry weight $\mathrm{m}^{-2} \mathrm{~d}^{-1}$ ) (Crabbe \& Smith, 2002). Flow rates for the area during the August sampling times were approximately $10-20 \mathrm{~cm} \mathrm{~s}^{-1}$ (Bell unpub. dat.). The coral cover is currently about $30-40 \%$ and has been declining in the last 5-6 years; however, sponges are abundant across a range of habitats (Bell et 
Chapter 6 Temporal and spatial diversity patterns of sponge assemblages in the Wakatobi Marine National Park, Indonesia.

al., 2010). Bell and Smith (2004) were the first to quantify sponge abundance and diversity in the area recording 71 species from the Hoga reef.

The three sites surveyed were all on the vertical walls of reef close to Hoga Island within the archipelagos. In $200515 \times 1 \mathrm{~m}^{2}$ permanent quadrats were established hapharardly at three sites (five quadrats at each site) about $200 \mathrm{~m}$ apart and between 10-14 m deep on vertical bedrock. An initial visual assessment of the sites suggested that they had similar sponge assemblages in overall assemblage composition and habitat type. These quadrats were analysed annually in situ in early August from 2005-2009 by the author and J. Bell.

\subsubsection{DATA COLLECtion}

Sponges were counted in situ within each quadrat as well as documenting the biodiversity in photos using a digital camera. Prior to counting or taking pictures, silt was 'wafted' so that the encrusting sponges could be seen as they are normally covered by a layer of silt which collects on the wall. Small samples $\left(<1 \mathrm{~cm}^{3}\right)$ were collected of each new morpho-species (O.T.U's) of sponge and photographed both in situ and under the light microscope in the lab. Because all patches visible to the naked eye were counted some of the specimens were very small and delicate and overgrowing other sponges. Some of the samples were therefore difficult to collect without contamination from the surrounding sponges or they were very delicate and almost dissolved on attempt to collect them. All specimens were exported under permit back to New Zealand for initial taxonomic analysis and then sample identifications were checked with John Hooper at the Queensland Museum. All species have been identified to the lowest taxonomic resolution possible given the sampling limitations and there are likely to be many new species present as many biodiversity surveys now ignore sponges under $200 \mathrm{~g}$ as they are not big enough for biochemical analysis for pharmaceutical potential (J. Hooper pers. com.). Morphological data was not collected because prior work comparing species and functional variability found correlations with univariate but not multivariate data and there was high 
Chapter 6 Temporal and spatial diversity patterns of sponge assemblages in the Wakatobi Marine National Park, Indonesia.

inter-phyletic redundancy due to multiple functional roles being performed by a large number of morphologies and species (Bell, 2007b). 
Chapter 6 Temporal and spatial diversity patterns of sponge assemblages in the Wakatobi Marine National Park, Indonesia.

\subsubsection{STATISTICAL ANALYSIS}

The species richness and abundance were calculated as the mean number of species/number of sponges found per $\mathrm{m}^{2} \pm \mathrm{SE}$ across each of the three different sites for each of the different sampling times. PRIMER (ver. 6) with the PERMANOVA add-on was used to carry out a non-parametric equivalent of a univariate ANOVA (permutational MANOVA) on the number of species and abundance as individual response variables; this method calculates P-values using permutations, thus needs no assumption of a normal distribution for the data. The model used for the PERMANOVA design is shown in Table 6.1. PERMDISP was used to test for the homogeneity of beta diversity within and between the sites and within and between seasons. This was in order to understand if all three sites were actually part of one location ( $\alpha$ diversity) or if the three sites were different from each other and therefore representative of beta diversity ( $\beta$ diversity).

TABLE 6.1 PERMANOVA model design for the analysis of the uni- and multivariate species data in response to the listed factors.

\begin{tabular}{|l|l|l|l|}
\hline Factor & Abbreviation & Type & Levels \\
\hline Site & Si & Random & 3 \\
\hline Quadrat & Qu & Fixed & 15 \\
\hline Year & Ye & Fixed & 5 \\
\hline
\end{tabular}

Species accumulation curves were plotted using an interpolated SOBs curve for smoothing the actual number of O.T.U.'s observed against the number of individuals observed across all three sites over time and Chao 2 to simulate a curve including the unknown species in the area. The curves were generated from 50 randomisations.

Additive diversity partitioning was used to detect any differences in the number of species within quadrats, sites and the turnover between years. Diversity partitioning is an analytical procedure in which a measure of total species diversity is decomposed among spatial or temporal levels in a hierarchical 
Chapter 6 Temporal and spatial diversity patterns of sponge assemblages in the Wakatobi Marine National Park, Indonesia.

sampling design (Veech et al. 2002). For temporally structured sampling designs, total species diversity at the conclusion of an inventory can be broken down into $\alpha$-and $\beta$-components, such that $\alpha$-diversity corresponds to the average diversity within a sampling unit (i.e. a single quadrat), and measures of $\beta$-diversity correspond to average diversity among sampling units, either among quadrats, between sites, or between years. Thus, for the experimental design:

\section{Total diversity $\gamma=\alpha_{\text {quadrats }}+\beta_{\text {sites }}+\beta_{\text {region }}$}

Lande (1996) demonstrated that three of the most commonly employed measures of species diversity; species richness, the Shannon index, and the Simpson Index are amenable to additive partitioning because they exhibit strict concavity. This means that the overall value of that measure for a pool set of assemblages equals or exceeds the average diversity within assemblages. Each measure of diversity, provides different information regarding community structure. Species richness measures the number of species added to a survey for each sampling level. Species richness weights rare taxa equally with abundant taxa and is therefore highly sensitive to sampling effort (Lande, 1996). Even when sampling effort is consistent, richness is generally dominated by rare taxa. The Shannon index measures the degree to which richness and evenness changes across sampling levels and is sensitive to rarer species (Veech et al. 2002). The Simpson Index has more bias to the common species and therefore both indexes will be used. Another attractive property of diversity partitioning is that the technique does not require rarefaction of the data prior to analyses, so differences in sampling effort among replicates within a given level do not bias partitions (Veech et al. 2002; Crist et al. 2003). This analysis was performed using PARTITION 3.0, a computer program described in detail by Summerville et al. (2003).

Differences in alpha diversity were measured initially through exploratory analysis of sample and individual-based rarefaction curves for each year group separately using the Chao 2 estimator and an interpolated Sobs (interpolated 
Chapter 6 Temporal and spatial diversity patterns of sponge assemblages in the Wakatobi Marine National Park, Indonesia.

using 50 runs of the species observed data) (Gotelli \& Colwell, 2001; Chao et al., 2005). Sample-based curves were calculated using EstimateS 8.2 (Colwell, 2009). Chao 2 estimator uses presence-absence data and incorporates the effect of unseen shared species by incorporation of the total number of species, the total number of singletons (species which only occur once) and the total number of doubletons (species which only occur twice) within a sample which increases accuracy of the curve (Chao et al., 2005).

PRIMER was used to carry out Ordination by non-metric Multi-Dimensional Scaling (MDS) on a dissimilarity matrix created from a Bray-Curtis similarity analysis to compare species between the three sites and between years to look for temporal and spatial patterns. The species data were $\log _{(\mathrm{x}+1)}$ transformed to reduce the influence of rare species. This is because even though rare species are important, it is often the common species that are having the most influence on the functioning of the ecosystem through their dominance of space (Gaston, 2010). MDS plots were created for the complete data set, and also for the sub groups in relation to site and year.

SIMPER analysis was used to elucidate the relative contribution of each species to the average Bay-Curtis similarity between the three sites and also the dissimilarity between the five years. This method determines which species drive any differences between the groups.

PERMANOVA was used to distinguish which factors or interacting factors were significant in determining the spatial and temporal differences between the sponge assemblages. The model used for the PERMANOVA design is the same as for the univariate data in Table 6.1. The test statistic for PERMANOVA is the pseudo F-ratio, where a large pseudo F-ratio indicates that a factor such as location or year groups the quadrats closer to one another in multivariate space than they are to random distribution and therefore differ in assemblage composition. The significance of the pseudo F-ratio is tested using a permutation test that randomly shuffles the sample labels within and among groups and calculates the pseudo F-ratio for 9999 arbitrary reassignments of the Bray Curtis resemblance matrices for species diversity/morphological 
Chapter 6 Temporal and spatial diversity patterns of sponge assemblages in the Wakatobi Marine National Park, Indonesia.

assemblages. The pseudo F-ratios of these randomly assigned communities are then compared to the pseudo F-ratio of the observed community to calculate the significance level of the test (Anderson, 2001).

\subsection{RESULTS}

One hundred and thirty six sponge O.T.U.'s (sponge species and entities identified to highest taxonomic level possible) across thirty families were recorded across the three sites over the five years. Some of the common species are shown in Fig. 6.2. Of the 136 0.T.U.'s, 73 were identified to at least genus level and are listed in Table 6.2. Some of the O.T.U.'s match samples of undescribed yet cataloged species in J. Hoopers collection at the Queensland Museum, Brisbane, Australia; for example Dysidea sp. 2338 in Fig. 6.2.f.

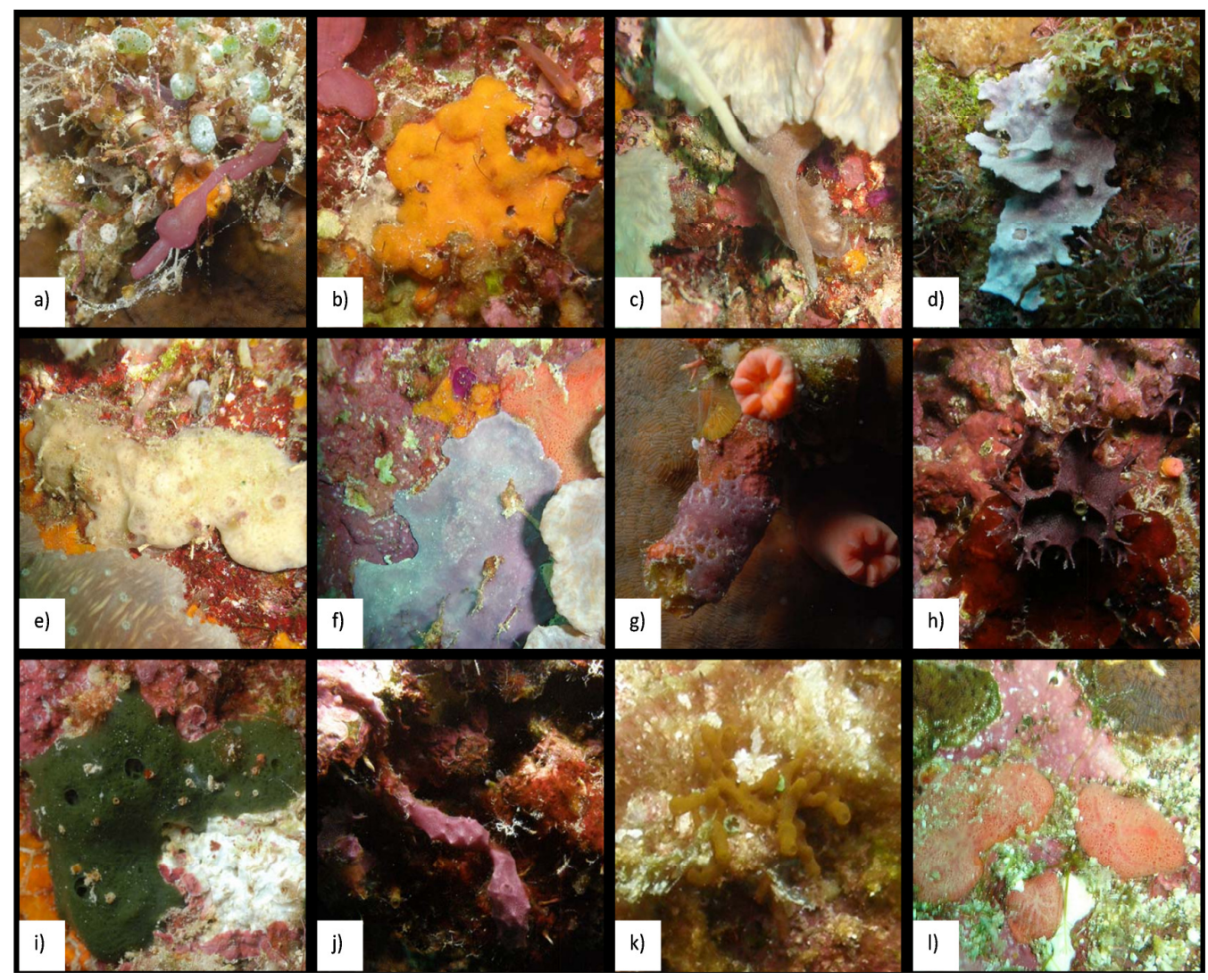

Fig. 6.2 Some of the common sponge species found within the Hoga sites. a) Pericharax sp. b) Protosuberites sp. c) Haliclona sp. d) Ecionemia c.f. acervus (Bowerbank, 1864) e) Petrosia (Petrosia) sp. f) Dysidea sp. 2328 g) Clathrina sp. h) Chelonaplysilla sp. i) Chalinula milnei (de Laubenfels, 1954) j) Pseudoceratina sp. k) Tedania sp. I) Tetillade sp. 
Chapter 6 Temporal and spatial diversity patterns of sponge assemblages in the Wakatobi Marine National Park, Indonesia.

Table 6.2 Sponge species identifications from the study sites at Hoga. Where a species name has a number after it these were undescribed species with voucher specimens in J. Hoopers database at the Queensland museum, Brisbane. The ID numbers were assigned for this study and match the voucher specimens held at VUW.

\begin{tabular}{|c|c|c|c|}
\hline Species & ID & Species & ID \\
\hline Aaptos sp. & 2 & Crella sp. & 77 \\
\hline Acanthella sp. & 23 & Cribochalina sp. & 65 \\
\hline Acanthella sp. & 38 & Cribrochalina sp. & 7 \\
\hline Acanthella sp. & 62 & Dendrilla sp. & 15 \\
\hline Agelas sp. & 64 & Desmacella sp. & 34 \\
\hline Agelas sp. & 94 & Dysidea sp. 2328 & 9 \\
\hline Axinella sp. & 43 & Dysidea sp. 17 & 17 \\
\hline Axinyssa sp. & 22 & Ecionemia c.f. acervus (Bowerbank, 1864) & 8 \\
\hline Biemna sp. & 27 & Eurypon sp. & 79 \\
\hline Calcareous sp. undet. & 14 & Gelliodes sp. & 39 \\
\hline Calcareous sp. undet. & 96 & Haliclona sp. & 6 \\
\hline Callyspongia sp. & 45 & Haplosclerina Sub order sp. undet. & 20 \\
\hline Chalinula milnei (de Laubenfels, 1954) & 4 & Hymedesmia sp. & 12 \\
\hline Chalinula nematifera (de Laubenfels, 1954) & 55 & lotrochota sp. & 71 \\
\hline Chalinula sp. & 50 & Jaspis sp. & 3 \\
\hline Chelonaplysilla sp. & 5 & Jaspis splendens & 33 \\
\hline Chelonaplysilla sp. & 40 & Leucetta sp. & 49 \\
\hline Chelonaplysina sp. & 108 & Leuconia sp. & 121 \\
\hline Cinachyrella c.f. australiansis (Carter, 1886) & 47 & $\begin{array}{l}\text { Liosina c.f. granularis (Kelly Borges \& } \\
\text { Bergquist, 1988) }\end{array}$ & 36 \\
\hline Cinachyrella sp. & 67 & Monanchora unguiculata (Dendy, 1922) & 66 \\
\hline Cinachyrella sp. & 102 & Myrmekioderma granulatum (Esper, 1794) & 105 \\
\hline Clathria (Microciona) mima (de Laubenfels, 1954) & 21 & Neopetrosia sp. & 31 \\
\hline $\begin{array}{l}\text { Clathria (Microcionia) c.f. lizardensis (Hooper, } \\
\text { 1996) }\end{array}$ & 41 & Niphates sp. & 19 \\
\hline Clathria (Microcionia) sp. & 18 & Niphates sp. & 78 \\
\hline Clathria (Thalysias) sp. c.f 2322 & 46 & Niphates sp. & 126 \\
\hline Clathria sp. & 16 & Niphates sp. c.f. 4514 & 60 \\
\hline Clathrina sp. & 24 & Pericharax sp. & 10 \\
\hline Clathrina sp. & 25 & Petrosia (Petrosia) sp. & 13 \\
\hline Clathrina sp. & 74 & Placospongia melobesioides (Gray, 1867) & 35 \\
\hline Clathrina sp. & 83 & Protosuberites sp. & 1 \\
\hline Clathrina sp. & 84 & Pseudoceratina sp. & 63 \\
\hline Clathrina sp. & 95 & Suberites sp. & 32 \\
\hline Clathrina sp. & 103 & Sycon sp. & 73 \\
\hline Clathrina sp. & 104 & Tedania sp. & 123 \\
\hline Cliona orientalis (Thiele, 1900) & 26 & Tetillade sp. & 11 \\
\hline Cliona sp. & 109 & Topsentia sp. & 82 \\
\hline Cliona sp. & 110 & Xestospongia sp. & 68 \\
\hline Cliona sp. & 122 & & \\
\hline
\end{tabular}


Chapter 6 Temporal and spatial diversity patterns of sponge assemblages in the Wakatobi Marine National Park, Indonesia.

The mean number of O.T.U.'s $\mathrm{m}^{-2}$ at different sites and between years are shown in figure 6.3. The mean O.T.U. richness differed significantly between the sites (Pseudo $\mathrm{F}=9.9263 \mathrm{P}=0.0003$ ), but not between the years using a permutational MANOVA. O.T.U. richness $\left( \pm\right.$ S.E) was calculated as $20.24( \pm 1.44)$ sponges $\mathrm{m}^{-2}$ at site $\mathrm{A}, 21.80( \pm 1.30)$ sponges $\mathrm{m}^{-2}$ at $\mathrm{B}$ and $24.08( \pm 1.34)$ sponges $\mathrm{m}^{-2}$ at $\mathrm{C}$.

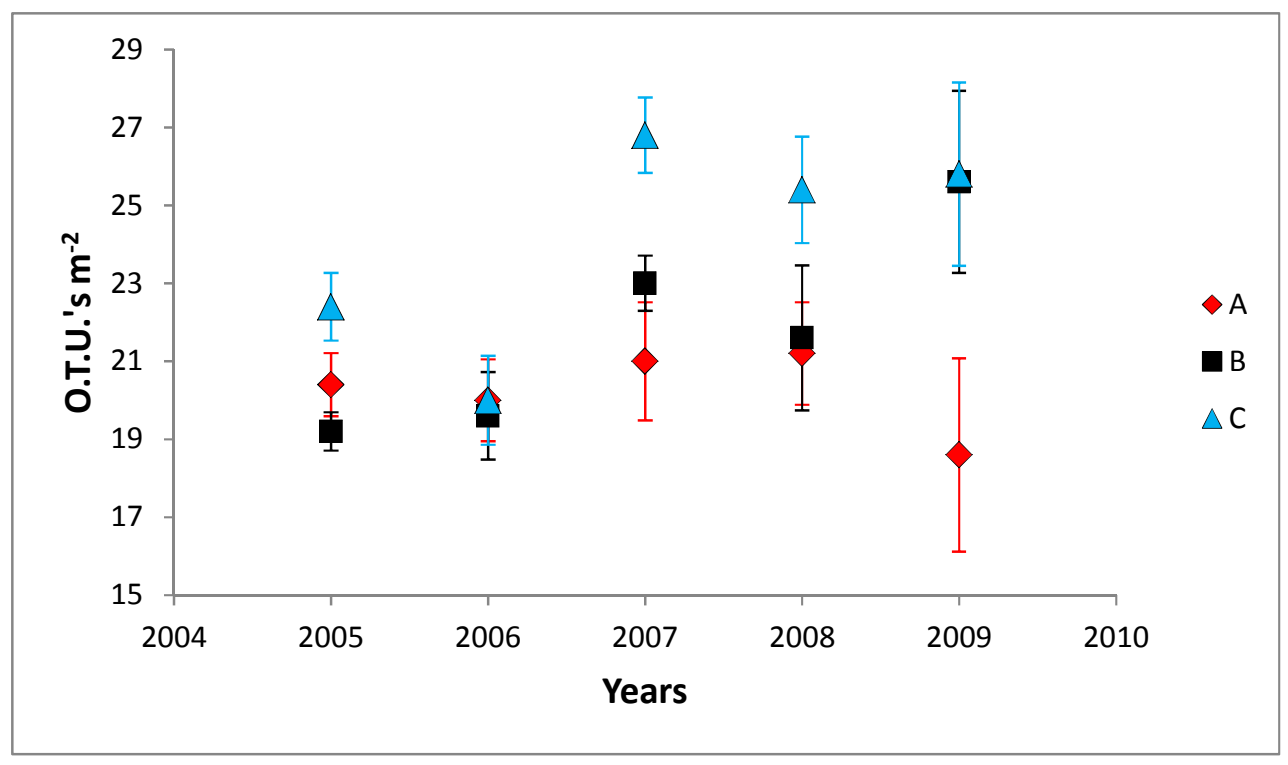

Fig. 6.3 Mean sponge species/entities per $\mathrm{m}-2 \pm \mathrm{SE}$ at the three Hoga sites $(A, B$, and $C$ ) in August 2005-2009. O.T.U. stands for operational taxonomic units.

The mean abundance of sponges found per $\mathrm{m}^{-2}$ is shown in figure 6.4. Site (pseudo $\mathrm{F}=24.531 \mathrm{p}=0.0001$ ) and year (pseudo $\mathrm{F}=4.0614 \mathrm{p}=0.0448$ ) and the interaction of site*year (pseudo $\mathrm{F}=2.8461 \mathrm{p}=0.0086$ ) were significant factors explaining the differences in sponge abundance using a permutational MANOVA. This means that the sites all showed significant annual changes, but the magnitude or direction of the change differed between the sites. The mean abundance of sponges per $\mathrm{m}^{-2}$ for each site (across all years) $( \pm \mathrm{SE}$ ) was 187.48 $( \pm 23.64)$ sponges $\mathrm{m}^{-2}$ at site $\mathrm{A}, 129.12( \pm 14.08)$ sponges $\mathrm{m}^{-2}$ at site $\mathrm{B}$ and 189.84 $( \pm 19.53)$ sponges $\mathrm{m}^{-2}$ at site $\mathrm{C}$. 
Chapter 6 Temporal and spatial diversity patterns of sponge assemblages in the Wakatobi Marine National Park, Indonesia.

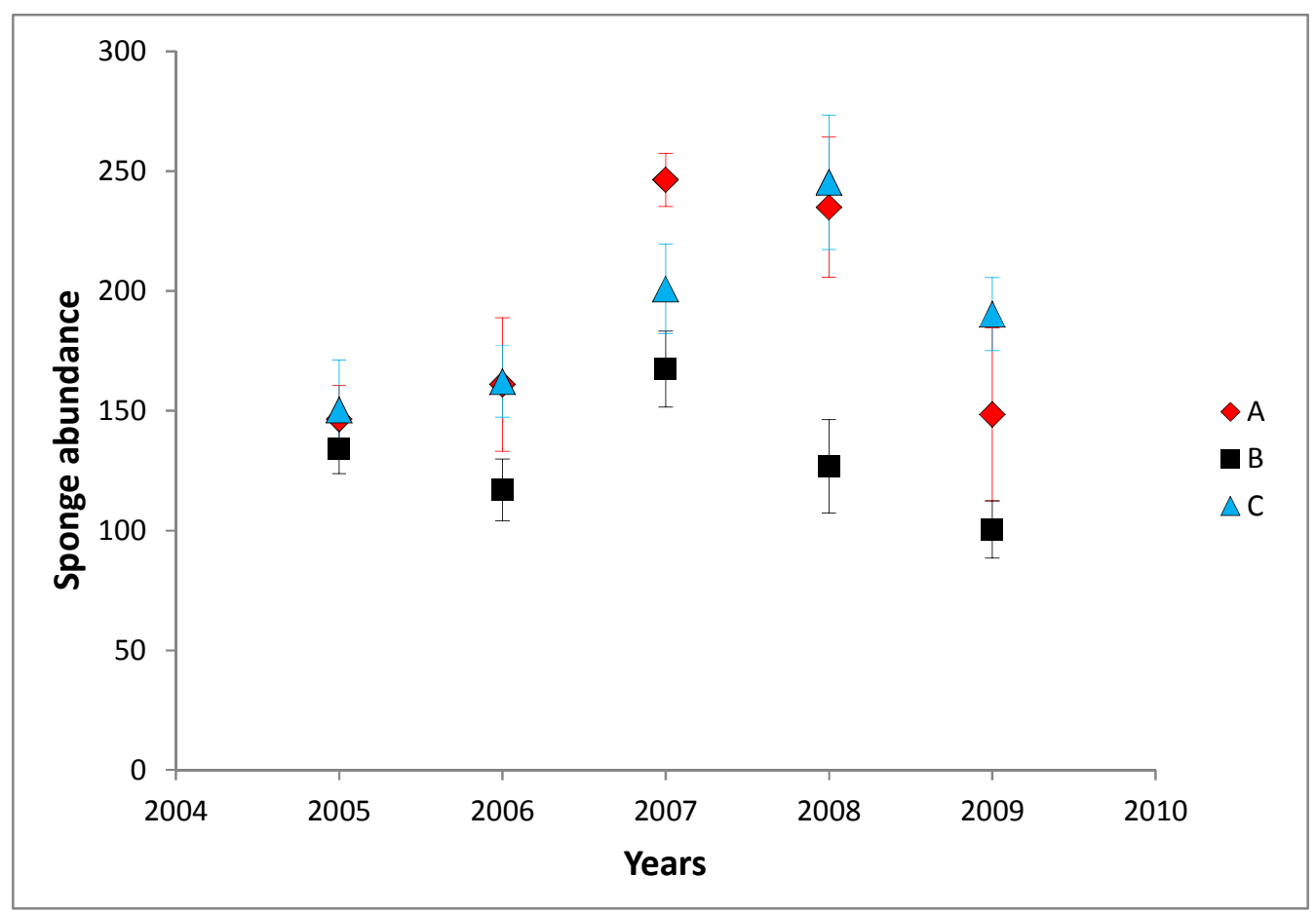

Fig. 6.4 Mean No. of sponges per $m^{-2} \pm S$.E at the three Hoga sites (A, B and C) from 2005-2009.

Species accumulation cures were plotted using an interpolated SOBs curve for smoothing the actual number of O.T.U.'s observed against the number of individuals observed across all three sites over time and Chao 2 to simulate a curve including the unknown species in the area (figure 6.5). None of the curves reached an asymptote and over the $15 \mathrm{~m}^{2}$ surveyed between 63 species (in 2006) and 86 species (in 2007) were observed within a sampling season across all sites although the number of sponge patches varied between 1861 (in 2005) and 2741 (in 2007). 
Chapter 6 Temporal and spatial diversity patterns of sponge assemblages in the Wakatobi Marine National Park, Indonesia.

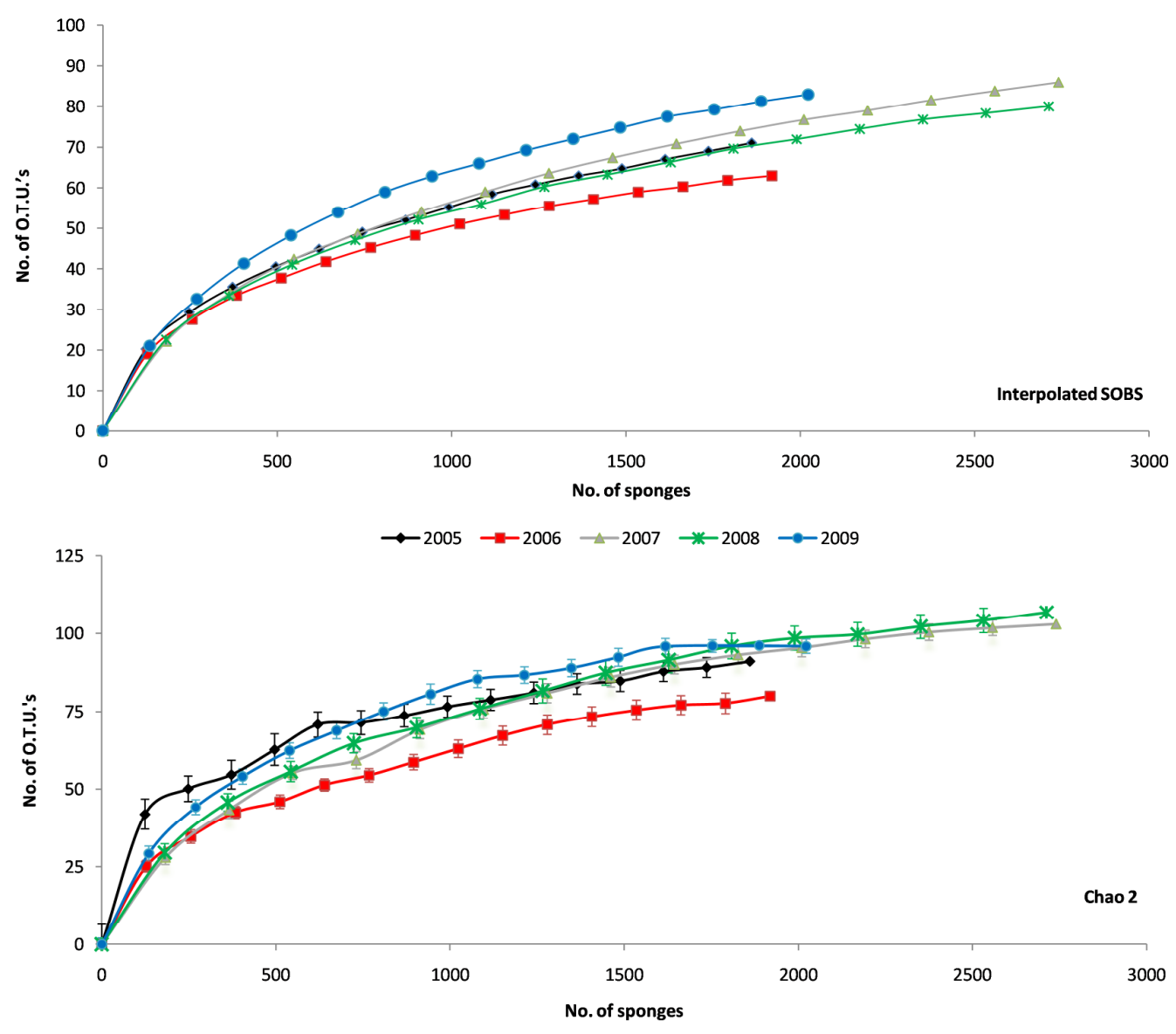

Fig. 6.5 Interpolated SOBS and Chao 2 Curves 2005-2009 with S.E for Hoga sponge O.T.U's and abundance of individuals across all three sites. The curves were generated from 50 randomisations.

Additive partitioning of species richness, Simpson and Shannon Index's across the three sites divided into years were compared (figure 6.6). The additive partitioning showed that for species richness the betaregion component was the most important factor explaining $50-60 \%$ of the variation and therefore turnover of species between years. The alpha $\mathrm{quadrat}_{\text {component contributed }}$ between $20-30 \%$ of the species richness and the betasite component contributed a similar percentage. Therefore there is a similar level of variability within a site compared to the variability between the three sites overall. There were no significant differences between years for species richness or for the Simpson Index. The Simpson Index shows that across all years the alpha $a_{\text {quadrat }}$ component 
Chapter 6 Temporal and spatial diversity patterns of sponge assemblages in the Wakatobi Marine National Park, Indonesia.

contributed over $80 \%$ of the total variation in biodiversity and therefore there was a high level of variation between quadrats.. For the Shannon Index, the alphaquadrat component from 2006 -2009 contributed over 78\% of the diversity with about $10 \%$ contributed by the betasite and the betaregion component. There was a shift from $60 \%(\mathrm{p}=0.06)$ contribution of the alphaquadrat component in 2005 to the close to $80 \%$ in 2006 with a corresponding change of the betaregion component from about $30 \%$ to less than $10 \%$. The betasite component remained consistent across all five years.
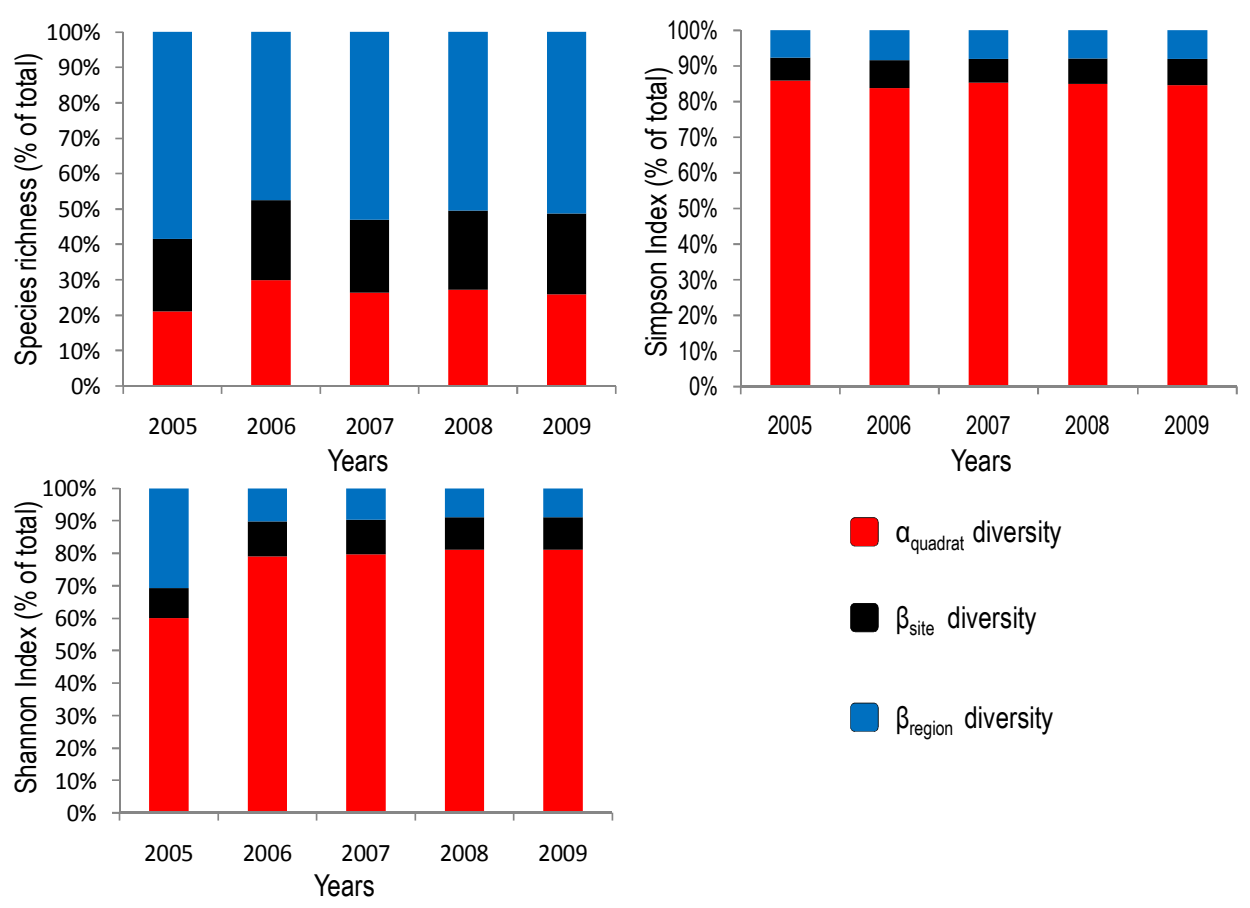

$a_{\text {audrat }}$ diversity

$\beta_{\text {site }}$ diversity

$\beta_{\text {region }}$ diversity

Figure 6.6 Additive partitioning of the diversity of Hoga sponge assemblages into alpha quadrat beta $_{\text {site }}$ beta $_{\text {region }}$ components by species richness, Simpson Index and Shannon Index across all three sites.

The MDS plot for the species assemblage data shows that there are differences between the sponge assemblages at the three sites sites and between years (Figure 6.7). Site A is more similar to B and than to C, while B and C are more similar to each other. The assemblage composition of all sites changed from 2005 to 2009 in the same direction. The 2009 assemblages are separate from the other years and also show more variability within the year across all three sites. 
Chapter 6 Temporal and spatial diversity patterns of sponge assemblages in the Wakatobi Marine National Park, Indonesia.

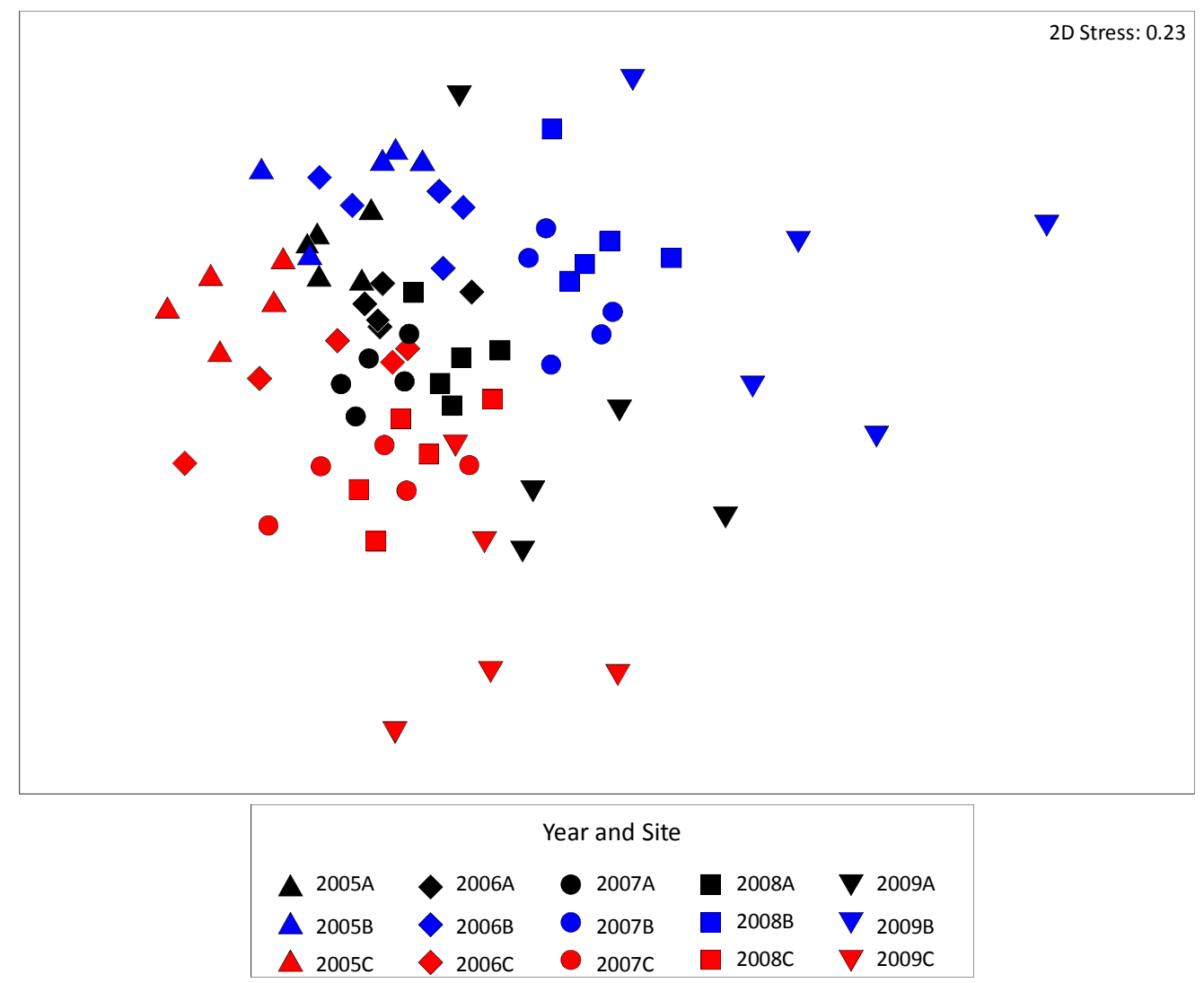

Figure 6.7 Multi-dimensional scaling (MDS) plot of sponge assemblages based on the species diversity data at the three sites A, B, \& C in August 2005-2009. Each point represents a single $1 \mathrm{~m}^{2}$ quadrat assemblage. The data has been log $(x-1)$ transformed from a Bray Curtis matrix.

Table 6.3 shows that there were were significant differences in the sponge assemblages due to Site, Year, the interaction term Site*Year and Quadrat (nested in site) using PERMANOVA.

Table 6.3. PERMANOVA results of the species assemblage data. Significant factor values are marked in bold. Unique permutations were observed out of 1000 .

\begin{tabular}{|l|l|l|l|l|l|l|}
\hline Source & $\begin{array}{l}\text { Degrees of } \\
\text { freedom }\end{array}$ & $\begin{array}{l}\text { Sums of } \\
\text { squares }\end{array}$ & $\begin{array}{l}\text { Expected } \\
\text { mean of } \\
\text { squares }\end{array}$ & Pseudo-F & P(perm) & $\begin{array}{l}\text { Unique } \\
\text { perms }\end{array}$ \\
\hline Site & $\mathbf{2}$ & 14530 & 7265 & 16.108 & 0.0001 & 9925 \\
\hline Year & 4 & 23111 & 5777.7 & 6.0529 & 0.0001 & 9935 \\
\hline Quadrat(Site) & 12 & 33837 & 2819.7 & 6.252 & 0.0001 & 9764 \\
\hline Site*Year & $\mathbf{8}$ & 7636.2 & 954.53 & 2.1164 & $\mathbf{0 . 0 0 0 1}$ & 9832 \\
\hline Residual & 48 & 21649 & 451.02 & & & \\
\hline Total & 74 & $1.0076 \mathrm{E}-5$ & & & & \\
\hline
\end{tabular}


Chapter 6 Temporal and spatial diversity patterns of sponge assemblages in the Wakatobi Marine National Park, Indonesia.

SIMPER analysis showed consistency in the presence and abundance of six O.T.U's at site A and seven species at sites B \& C. These species were able to explain over $60 \%$ of the similarity within each of the sites across the five years surveyed (Table 6.4). The full SIMPER analysis results are in Appendix 6.6.

Table 6.4 O.T.U's which are important in explaining the similarity within Sites across all years sampled. The O.T.U's are listed with their percentage contribution towards explaining the similarity within each of the sites.

\begin{tabular}{|c|c|c|c|}
\hline Species & A \% Contribution & B \% Contribution & C \% Contribution \\
\hline Protosuberites sp. (1) & 17.52 & 16.46 & 9.5 \\
\hline Ecionemia c.f. acervus (8) & 11.39 & 6.38 & 9.28 \\
\hline Sycon sp. (73) & 10.23 & 7.15 & 11.86 \\
\hline Pericharax sp. (10) & 9.43 & 9.89 & 11.19 \\
\hline Haplosclerina Suborder sp. (20) & 8.57 & 8.14 & N/A \\
\hline Dysidea sp. 17 (17) & 7.89 & $\mathrm{~N} / \mathrm{A}$ & 7.87 \\
\hline Chelonaplysilla sp. (5) & N/A & 9.3 & $\mathrm{~N} / \mathrm{A}$ \\
\hline Dysidea sp. 2328 (9) & $\mathrm{N} / \mathrm{A}$ & 5.76 & $\mathrm{~N} / \mathrm{A}$ \\
\hline Haliclona sp. (6) & $\mathrm{N} / \mathrm{A}$ & $\mathrm{N} / \mathrm{A}$ & 6.94 \\
\hline Petrosia (Petrosia) sp. (13) & $\mathrm{N} / \mathrm{A}$ & $\mathrm{N} / \mathrm{A}$ & 4.6 \\
\hline
\end{tabular}

Twenty to twenty-five O.T.U.'s were needed to explain at least $60 \%$ of the dissimilarity between sites using SIMPER. Some of the important species responsible for the differences between sites and therefore the variability were the same as those listed in Table 6.4. Protosuberites sp. (1) was responsible for $3.64 \%$ of the dissimilarity between sites $\mathrm{A}$ and $\mathrm{B}, 4.78 \%$ between sites $\mathrm{A}$ and $\mathrm{C}$ and 3.49\% between A and C. Some species were only found at one of the sites and not at the other, for example, Tedania sp. (123) was only found at site $\mathrm{C}$ and not at site B and was responsible for $2.3 \%$ of the dissimilarity. When data were examined further Tedania sp. (123) was not observed at any of the sites until 2008 and 2009 and therefore it explains some of the temporal variability between years; for example $2.4 \%$ of the dissimilarity between 2005 and 2009, $2.61 \%$ between 2006 and 2009, and 2.45\% between 2007 and 2009. Between 
Chapter 6 Temporal and spatial diversity patterns of sponge assemblages in the Wakatobi Marine National Park, Indonesia.

17 and 25 O.T.U.'s were needed to explain at least $60 \%$ of the variation between the years sampled. The abundance of Sycon sp. (73) was variable between years and therefore it provided an important contribution to the dissimilarity between years sampled. For example, Sycon sp. (73) contributed $8.35 \%$ to the differences between 2005 and 2006 across all sites, 10.15\% between 2005 and 2007 and $4.10 \%$ between 2007 and 2008. This is because it was a rare species in 2005 with only 3 sponges recorded from only one quadrat in out of the fifteen surveyed in 2005 and in the other years it was much more common with over 18 sponge patches per $\mathrm{m}^{2}$ in 2009 over 10 sponge patches in 2006, over 39 in 2007 and over 35 in 2008. Figure 6.8 shows the mean shifts in abundance of some of the important species across all sites over the five year study period.

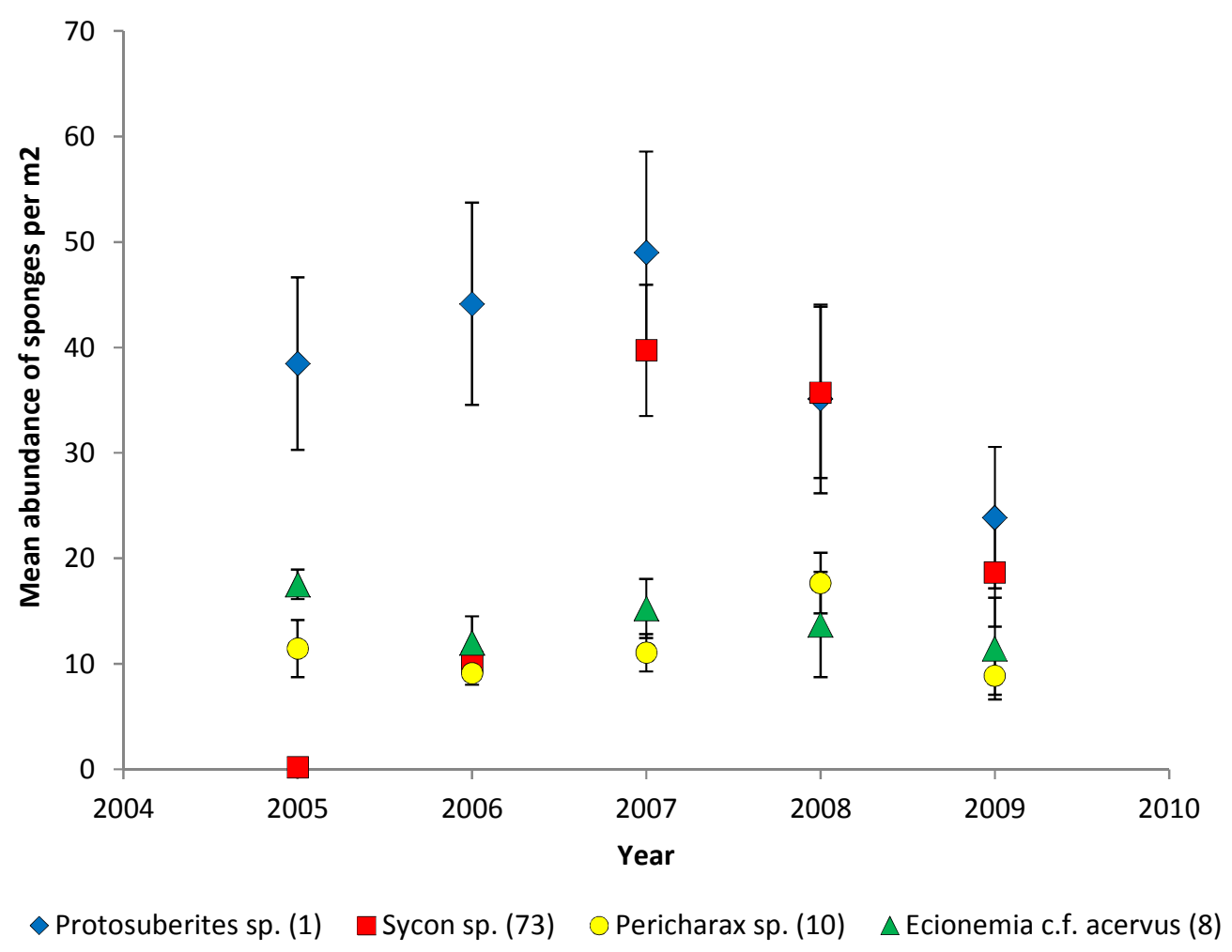

Figure 6.8 Annual mean abundances in August 2005-2009 ( \pm S.E) of Protosuberites sp. (1), Sycon sp. (73), Pericharax sp. (10) and Ecionemia c.f. acervus (8) within Hoga. 
Chapter 6 Temporal and spatial diversity patterns of sponge assemblages in the Wakatobi Marine National Park, Indonesia.

\subsection{DISCUSSION}

This study compared the same areas over time to determine the sponge assemblage changed annually and across local spatial scales. There were significant changes in the sponge assemblage composition and abundance at the quadrat and site scale and between years. However, the partitioning of sponge assemblage diversity remained consistent between years for the species richness, Shannon Index and Simpsons Index. The consistency of diversity partitioning means that although the abundances of the different O.T.U's did vary between years, the ratio of the diversity within the quadrats compared to the site and region remains consistent and therefore the annual variation in beta diversity appears to be low.. This means that the actual species abundances were significantly different between sites and years, however, the relative number of species within the individual quadrats, sites, and the location remain consistent between years even though different species and abundances are involved.

One hundred and thirty six sponge O.T.U.'s, across thirty families, and at least 73 genre were recorded across the three sites over the five years of surveys. Many of the O.T.U.'s are likely to be undescribed species, probably due to the high biodiversity of the Indo-Pacific (van Soest, 1989; Edinger et al., 2000; Roberts et al., 2002; Karlson et al., 2004; de Voogd et al., 2006; de Voogd \& Cleary, 2008).

The species richness (number of O.T.U's) varied significantly between sites, but not between the years. Studies investigating spatial beta diversity over larger scales in the Indo-Pacific have found that sponge biodiversity strongly correlates with environmental variables such as depth, habitat heterogeneity, substratum angle, and the degree of human settlement (Cleary et al., 2005; de Voogd et al., 2006; Bannister et al., 2010; Powell et al., 2010). However, it is hard to separate the environmental factors as there are likely to be interactions between factors, for example depth and substratum. Environmental gradients will always incorporate some spatial component through the distance apart of 
Chapter 6 Temporal and spatial diversity patterns of sponge assemblages in the Wakatobi Marine National Park, Indonesia.

the sampling sites so a variance partitioning technique described by Borcard et al., (1992) has been used previously to separate the environmental variables from their spatial components in some studies (Cleary et al., 2005; de Voogd et al., 2006).

All the quadrats in this study were at approximately the same depth, and along the same wall of reef a couple of hundred meters apart and therefore similar distances from human settlement. The sampling sites were all vertical reef, however, there were varying degrees of overhangs and cracks within the quadrats therefore there will be some inter-site differences in small-scale conditions. For example, there are differences in other phyla between the sites such as coralline algae (about $45 \%$ coverage at site $\mathrm{C}$ and $20 \%$ coverage at sites A \& B), hard coral (60\% at site B, $45 \%$ at site A and $20 \%$ at site C) and soft coral percentage cover (about $15 \%$ at sites A \& C and $<5 \%$ at site B) (Hay, 2008). Sponge diversity has been found to be significantly positively correlated with hard coral cover in the same study area in a previous study over a range of habitats (Powell et al., 2010). However, in this study sponge abundance and diversity was at its lowest in 2006 at site B, which had the highest coral coverage that year. Total percentage coverage for sponges, and the other sessile phyla present did not change significantly from 2006 to 2007 (Hay, 2008), even though the numbers of sponges counted in situ changed significantly in abundances in this study.

It is important to take into consideration that the percentage cover in Hay (2008) was calculated from photo quadrats and any shaded areas or silt covered areas were not included therefore it would be difficult to see in a photograph all the small cryptic sponges that dominate at these sites as they are often under ledges and spurs as well as being in cracks in the rock (Fig. 6.9). The number of sponges could have also increased through fragmentation for example by predation from fish; however predation also decreases the abundance of sponge patches as well when a patch is consumed completely (Wulff, 1994). Fish have been observed feeding on sponges in the study area, 
Chapter 6 Temporal and spatial diversity patterns of sponge assemblages in the Wakatobi Marine National Park, Indonesia.

however no species specific data is currently available to quantify the impact fish may have on the sponge assemblages.

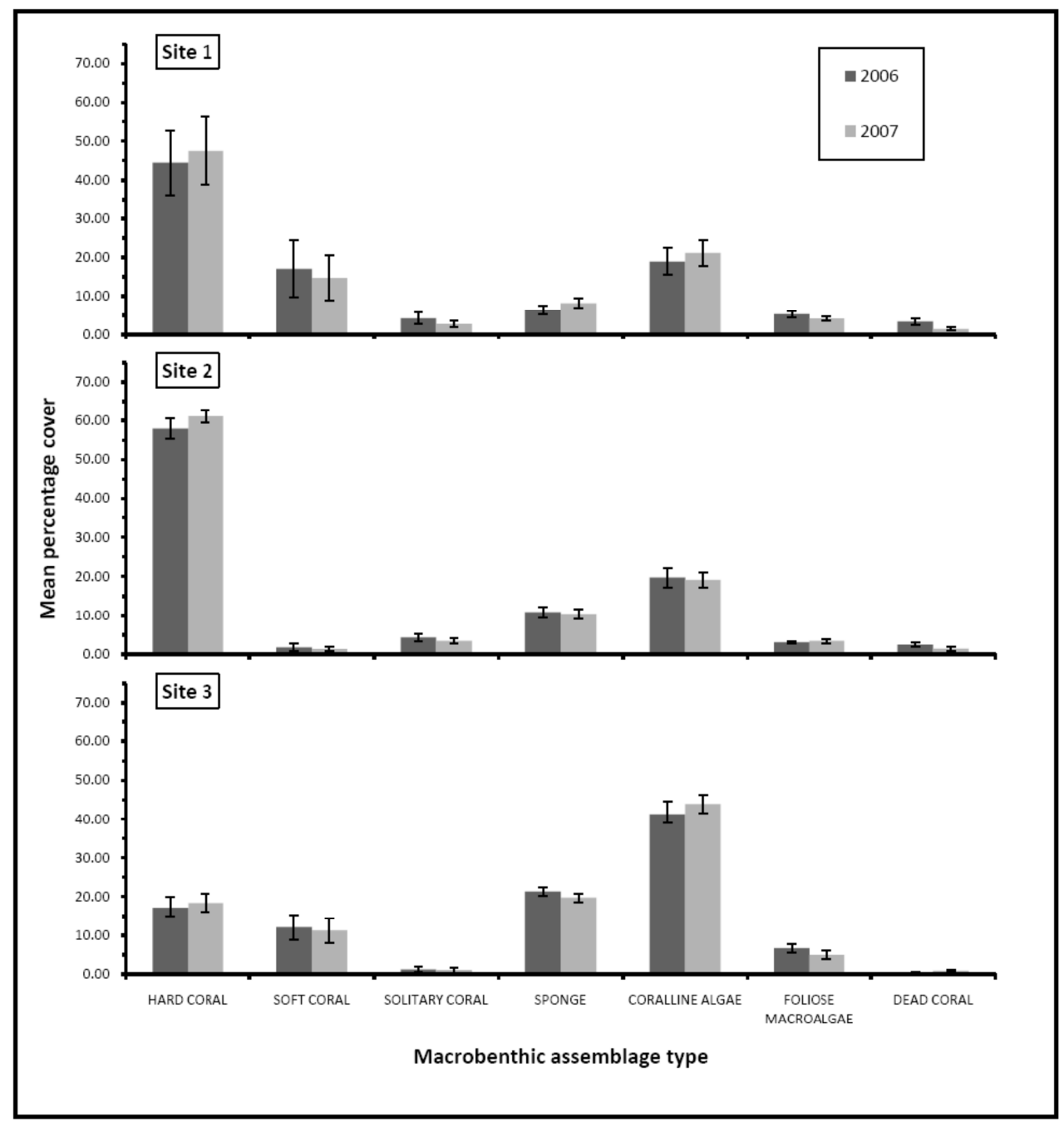

Fig. 6.9 Mean percentage cover ( \pm S.E) of macrobenthic assemblage types identified form photographs at the three study sites, Hoga, Indonesia in August 2006 and 2007 (Hay, 2008).

All of the sites were on vertical bedrock, however, the reef wall did have numerous spurs and mini caves that change the angle of the substrate and habitat complexity as well as the micro-environmental conditions. At Hoga it has been found that substratum angle can have an effect on overall benthic composition, but not directly on sponge densities across a range of hard bottomed benthic habitats (Powell et al., 2010). At sites with high levels of sedimentation (20.1g.d.wt.m ${ }^{-2} \mathrm{~d}^{-1}$ compared with 7.5g.d.wt.m $\mathrm{m}^{-2} \mathrm{~d}^{-1}$ at Hoga 
Chapter 6 Temporal and spatial diversity patterns of sponge assemblages in the Wakatobi Marine National Park, Indonesia.

(Crabbe \& Smith, 2002)) vertical surfaces had higher species richness then inclined or horizontal, but in agreement with Powell (2010) and Bell \& Smith (2004) there was no significant difference at the Hoga sites between different substratum angles. However, the habitat structural complexity through microenvironmental variation in the substrate could explain some of the spatial variation the sites as it will influence local water flow dynamics and light availability, which both are factors known to influence sponges assemblages (Alcolado, 1994; Bell, 2002; Ávila \& Carballo, 2008). The high structural complexity of reef substrate is known to promote sponge abundance and diversity through increasing the surface area available for sessile organisms (Diaz et al., 1990). The majority of sponges within the WMNP are small and cryptic, and are found across a wide range of habitat types and can take advantage of the range of niches available due to the structural complexity of the habitat and therefore this will lead to more within site spatial variability.

There was a trend for the variability of sponge assemblages within the sites and across all sites to increase over the study period, which is clear from the MDS plot and the univariate species richness and abundance data. The majority of sponge species have been found to be randomly distributed in relation to other sponges at Hoga in the non-sedimented areas similar to the sites in this study (Bell et al., 2010). Beyond the random distribution, the causal relationships for these changes to a more variable structure are hard to explain without complementary environmental data for the area. A recent six year study in Mexico examining temporal changes in tropical sponge assemblages found that short and long-term fluctuations in sponge densities were linked to seasonal changes in wind patterns and large-scale structuring factors, such as El Niño (Carballo et al., 2008). There was a moderate El Niño in late 2006 (Sea Surface temperatures were $1^{\circ} \mathrm{C} \pm 0.1$ warmer than the three month running means) that led to drought conditions in Indonesia and many forest fires (Logan et al., 2008). There was an increase in both species richness and abundance from 2006 to 2007 across all three sites so potentially the assemblages could have been influenced by the El Niño conditions. Other biological factors could 
Chapter 6 Temporal and spatial diversity patterns of sponge assemblages in the Wakatobi Marine National Park, Indonesia.

also play a role in influencing the sponge abundances, such as disease, although there was no direct evidence within the quadrats. A fourteen year study in the Caribbean recorded the steady disappearance of some of the sponge species and attributed the losses to disease (Wulff, 2006). Sponge diseases are hard to track without continuous monitoring as sponges rapidly loose diseased parts or disappear altogether (Wulff, 2001). However, instantaneous observations show little evidence of sponges with disease.

Geographical patterns of species diversity and endemism are influenced by the relative rates of speciation and extinction (Reaka et al., 2008). The hotspot of biodiversity in Indonesia is the result of the combination of higher seawater temperatures, the stability of the environment, ecological diversification and the large size of the coral reef systems (in comparison to the Pacific) (Reaka et al., 2008). In species rich assemblages there are a variety of life strategies that can respond differently to shifts in environmental conditions and contribute to ecological functioning in various ways, however, there is also a high level of functional redundancy, which means that there are multiple species which can carry out the same functional roles increasing the stability of the assemblage (McCann, 2000; Tilman et al., 2006; Yachi \& Loreau, 2006). The abundances of each species required to carry out the same role within an ecosystem are not necessarily the same, therefore abundances of species can fluctuate in a system with functional redundancy and the ecosystem can remain stable. There is likely to be a high level of interphyletic redundancy within the sponge assemblages (Bell, 2007b), which may explain why the diversity within the quadrats, sites and local area remain stable. Species richness, Shannon, and Simpson diversity measures responded in different ways to diversity partitioning. Observed species richness was most affected by betaregion which accounted for over half the diversity within the area sampled. In contrast to species richness, Shannon and Simpson diversity was predominantly determined at the scale of individual quadrats, with site and regional differences having lesser importance. Therefore the variability with each site is higher than that between the sites.

This is the first study to use additive partitioning of biodiversity to study sponge 
Chapter 6 Temporal and spatial diversity patterns of sponge assemblages in the Wakatobi Marine National Park, Indonesia.

assemblages and the first study to investigate temporal partitioning of diversity in a marine context. The limitations of this survey are that only one habitat and depth were included across a small area (a few hundred metres along one reef) so it is not known if the partitioning of diversity across larger areas and different habitat types would hold similar patterns however this would be interesting to explore further. 
Chapter 6 Temporal and spatial diversity patterns of sponge assemblages in the Wakatobi Marine National Park, Indonesia.

\subsection{APPENDIX - SIMPER Similarity Percentages - Two-way analysis of SPECIES CONTRIBUTIONS OF SITE CROSSED WITH SEASON. (60\% CONTRIBUTION CUT OFF)}

Table 6.5 Species responsible for explaining the similarity across all sites in 2005. Average similarity between all quadrats in 2005 was $58.0 \%$

\begin{tabular}{|l|c|c|c|c|c|}
\hline Species & Av.Abund & Av.Sim & Sim/SD & Contrib. \% & Cum. \% \\
\hline Protosuberites sp. (1) & 3.26 & 9.32 & 2.76 & 16.07 & 16.07 \\
Ecionemia c.f. acervus (8) & 2.87 & 9.10 & 7.49 & 15.69 & 31.76 \\
Pericharax sp. (10) & 2.15 & 5.52 & 1.68 & 9.51 & 41.28 \\
Petrosia (Petrosia) sp. (13) & 1.95 & 4.81 & 1.72 & 8.30 & 49.58 \\
Chelonaplysilla sp. (5) & 1.51 & 4.29 & 1.54 & 7.40 & 56.98 \\
Haliclona sp. (6) & 1.65 & 3.82 & 1.62 & 6.59 & 63.57 \\
\hline
\end{tabular}

Table 6.6 Species explaining the similarity across all sites in 2006. Average similarity between all quadrats in 2006 was $57.31 \%$

\begin{tabular}{|l|c|c|c|c|c|}
\hline Species & Av.Abund & Av.Sim & Sim/SD & Contrib. \% & Cum. \% \\
\hline Protosuberites sp. (1) & 3.50 & 10.41 & 4.88 & 18.15 & 18.15 \\
Sycon sp. (73) & 2.30 & 6.91 & 4.06 & 12.06 & 30.22 \\
Pericharax sp. (10) & 2.16 & 6.34 & 2.28 & 11.06 & 41.28 \\
Ecionemia c.f. acervus (8) & 2.08 & 4.71 & 1.15 & 8.21 & 49.49 \\
Dysidea sp. 17 (17) & 1.73 & 4.39 & 2.20 & 7.67 & 57.16 \\
Haplosclerina Sub order sp.(20) & 1.55 & 3.69 & 1.21 & 6.44 & 63.60 \\
\hline
\end{tabular}

Table 6.7 Species explaining the similarity across all sites in 2007. Average similarity between all quadrats in 2007 was $58.16 \%$

\begin{tabular}{|l|c|c|c|c|c|}
\hline Species & Av.Abund & Av.Sim & Sim/SD & Contrib. \% & Cum. \% \\
\hline Sycon sp. (73) & 3.48 & 8.93 & 3.95 & 15.36 & 15.36 \\
Protosuberites sp. (1) & 3.54 & 8.59 & 2.75 & 14.76 & 30.12 \\
Pericharax sp. (10) & 2.36 & 5.69 & 4.47 & 9.78 & 39.89 \\
Ecionemia c.f. acervus (8) & 2.42 & 5.33 & 1.65 & 9.17 & 49.07 \\
Chelonaplysilla sp. (5) & 1.65 & 3.99 & 2.39 & 6.85 & 55.92 \\
Dysidea sp. 17 (17) & 1.64 & 3.1 & 1.15 & 5.33 & 61.24 \\
\hline
\end{tabular}

Table 6.8 Species explaining the similarity across all sites in 2008. Average similarity between all quadrats in 2008 was $57.71 \%$

\begin{tabular}{|l|c|c|c|c|c|}
\hline Species & Av.Abund & Av.Sim & Sim/SD & Contrib. \% & Cum. \% \\
\hline Sycon sp. (73) & 3.13 & 7.25 & 2.14 & 12.56 & 12.56 \\
Protosuberites sp. (1) & 3.15 & 7.24 & 3.66 & 12.54 & 25.1 \\
Pericharax sp. (10) & 2.78 & 7.02 & 4.53 & 12.16 & 37.26 \\
Haplosclerina Sub order sp. (20) & 1.86 & 4.33 & 3.82 & 7.5 & 44.76 \\
Chelonaplysilla sp. (5) & 1.66 & 4.13 & 1.82 & 7.16 & 51.92 \\
Ecionemia c.f. acervus (8) & 2.02 & 3.77 & 1.32 & 6.54 & 58.45 \\
Dysidea sp. 2328 (9) & 1.77 & 3.55 & 0.99 & 6.15 & 64.61 \\
\hline
\end{tabular}


Chapter 6 Temporal and spatial diversity patterns of sponge assemblages in the Wakatobi Marine National Park, Indonesia.

Table 6.9 Species explaining the similarity across all sites in 2009. Average similarity between all quadrats in 2009 was $45.84 \%$

\begin{tabular}{|l|c|c|c|c|c|}
\hline Species & Av.Abund & Av.Sim & Sim/SD & Contrib. \% & Cum. \% \\
\hline Haplosclerina Sub order sp. (20) & 2.29 & 5.9 & 5.27 & 12.87 & 12.87 \\
Protosuberites sp. (1) & 2.34 & 4.58 & 1.08 & 10 & 22.87 \\
Sycon sp. (73) & 2.21 & 4.28 & 1.08 & 9.34 & 32.21 \\
Pericharax sp. (10) & 1.87 & 3.59 & 1.19 & 7.82 & 40.03 \\
Dysidea sp. 2328 (9) & 1.68 & 3.2 & 1.27 & 6.98 & 47.01 \\
Dysidea sp. 17 (17) & 1.45 & 3.04 & 1.33 & 6.63 & 53.64 \\
Ecionemia c.f. acervus (8) & 1.64 & 2.58 & 0.92 & 5.62 & 59.27 \\
Haliclona sp. (6) & 1.37 & 1.95 & 0.93 & 4.25 & 63.51 \\
\hline
\end{tabular}

Table 6.10 Species explaining the differences between all sites in 2005 and 2006. Average percentage difference between 2005 and 2006 was $45.50 \%$

\begin{tabular}{|l|c|c|c|c|c|c|}
\hline Species & $\begin{array}{c}2005 \text { Av. } \\
\text { Abund }\end{array}$ & $\begin{array}{c}2006 \text { Av. } \\
\text { Abund }\end{array}$ & $\begin{array}{c}\text { Av. } \\
\text { Diss }\end{array}$ & $\begin{array}{c}\text { Diss/ } \\
\text { SD }\end{array}$ & $\begin{array}{c}\text { Contrib } \\
\%\end{array}$ & Cum.\% \\
\hline Sycon sp. (73) & 0.09 & 2.3 & 3.8 & 3.19 & 8.35 & 8.35 \\
Haliclona sp. (6) & 1.65 & 1.11 & 1.88 & 1.44 & 4.13 & 12.48 \\
Ecionemia c.f. acervus (8) & 2.87 & 2.08 & 1.86 & 0.99 & 4.09 & 16.57 \\
Petrosia (Petrosia) sp. (13) & 1.95 & 1.54 & 1.79 & 1.43 & 3.93 & 20.5 \\
Purple thin encrust. (16) & 1.16 & 0.14 & 1.72 & 1.29 & 3.78 & 24.28 \\
Haplosclerina Sub order sp. (20) & 1.12 & 1.55 & 1.63 & 1.52 & 3.59 & 27.87 \\
Protosuberites sp. (1) & 3.26 & 3.5 & 1.57 & 1.23 & 3.46 & 31.33 \\
Chalinula milnei (4) & 0.87 & 1 & 1.55 & 1.06 & 3.41 & 34.74 \\
Dysidea sp. 17 (17) & 1.57 & 1.73 & 1.52 & 1.16 & 3.34 & 38.08 \\
Pericharax sp. (10) & 2.15 & 2.16 & 1.51 & 1.05 & 3.33 & 41.41 \\
Dendrilla sp. (15) & 0.82 & 0.69 & 1.44 & 1.27 & 3.16 & 44.57 \\
Chelonaplysilla sp. (5) & 1.51 & 1.37 & 1.34 & 1.52 & 2.95 & 47.52 \\
Jaspis sp. (3) & 1.05 & 1.03 & 1.31 & 1.25 & 2.89 & 50.41 \\
Dysidea sp. 2328 (9) & 0.7 & 0.4 & 1.2 & 1.08 & 2.64 & 53.05 \\
Hymedesmia sp. (12) & 0.54 & 0.47 & 1.2 & 0.9 & 2.64 & 55.69 \\
Clathrina sp. (24) & 0.59 & 0.43 & 1.01 & 1.1 & 2.23 & 57.92 \\
Aaptos sp. (2) & 0.53 & 0.62 & 0.95 & 1.11 & 2.09 & 60.01 \\
\hline
\end{tabular}


Chapter 6 Temporal and spatial diversity patterns of sponge assemblages in the Wakatobi Marine National Park, Indonesia.

Table 6.11 Species explaining the differences between all sites in 2005 and 2007. Average percentage difference between 2005 and 2007 was $51.22 \%$

\begin{tabular}{|l|c|c|c|c|c|c|}
\hline Species & $\begin{array}{c}2005 \text { Av. } \\
\text { Abund }\end{array}$ & $\begin{array}{c}2007 \text { Av. } \\
\text { Abund }\end{array}$ & $\begin{array}{c}\text { Av. } \\
\text { Diss }\end{array}$ & $\begin{array}{c}\text { Diss/ } \\
\text { SD }\end{array}$ & $\begin{array}{c}\text { Contrib } \\
\%\end{array}$ & Cum.\% \\
\hline Sycon sp. (73) & 0.09 & 3.48 & 5.2 & 4.05 & 10.15 & 10.15 \\
Petrosia (Petrosia) sp. (13) & 1.95 & 0.4 & 2.6 & 1.77 & 5.08 & 15.23 \\
Clathrina sp. (84) & 0 & 1.34 & 2.07 & 1.01 & 4.04 & 19.27 \\
Haliclona sp. (6) & 1.65 & 1.3 & 1.57 & 1.24 & 3.07 & 22.34 \\
Dysidea sp. 17 (17) & 1.57 & 1.64 & 1.46 & 1.3 & 2.85 & 25.19 \\
Protosuberites sp. (1) & 3.26 & 3.54 & 1.41 & 1.23 & 2.75 & 27.94 \\
Dysidea sp. 2328 (9) & 0.7 & 0.6 & 1.39 & 1.03 & 2.71 & 30.65 \\
Jaspis sp. (3) & 1.05 & 0.83 & 1.31 & 1.24 & 2.56 & 33.21 \\
Hymedesmia sp. (12) & 0.54 & 0.54 & 1.28 & 0.95 & 2.5 & 35.71 \\
Pericharax sp. (10) & 2.15 & 2.36 & 1.26 & 1.07 & 2.46 & 38.17 \\
Ecionemia c.f. acervus (8) & 2.87 & 2.42 & 1.24 & 0.84 & 2.42 & 40.59 \\
Tetilidae sp. (11) & 0.28 & 0.88 & 1.23 & 1.26 & 2.41 & 43 \\
Dendrilla sp. (15) & 0.82 & 0.96 & 1.16 & 1.44 & 2.26 & 45.26 \\
Haplosclerina Sub order sp. (20) & 1.12 & 1.46 & 1.16 & 1.17 & 2.25 & 47.52 \\
Purple thin encrust. (16) & 1.16 & 0.48 & 1.15 & 1.03 & 2.24 & 49.76 \\
Chalinula milnei (4) & 0.87 & 0.54 & 1.12 & 1.11 & 2.18 & 51.94 \\
Aaptos sp. (2) & 0.53 & 0.63 & 1 & 1.19 & 1.96 & 53.9 \\
Clathrina sp. (24) & 0.59 & 0.45 & 0.88 & 1.03 & 1.73 & 55.62 \\
Callyspongia sp. (45) & 0.24 & 0.64 & 0.88 & 0.97 & 1.71 & 57.33 \\
Chelonaplysilla sp. (5) & 1.51 & 1.65 & 0.86 & 1.53 & 1.68 & 59.01 \\
Placospongia melobesioides(35) & 0.45 & 0.38 & 0.85 & 0.95 & 1.65 & 60.66 \\
\hline
\end{tabular}

Table 6.12 Species explaining the differences between all sites in 2006 and 2007. Average percentage difference between 2006 and 2007 was $46.65 \%$

\begin{tabular}{|l|c|c|c|c|c|c|}
\hline & 2007 & & & & \\
Species & $\begin{array}{c}2006 \text { Av. } \\
\text { Abund }\end{array}$ & $\begin{array}{c}\text { Av. } \\
\text { Abund }\end{array}$ & $\begin{array}{c}\text { Av. } \\
\text { Diss }\end{array}$ & Diss/SD & $\begin{array}{c}\text { Contrib } \\
\%\end{array}$ & Cum.\% \\
\hline Clathrina sp. (84) & 0 & 1.34 & 2.1 & 1.01 & 4.49 & 4.49 \\
Petrosia (Petrosia) sp. (13) & 1.54 & 0.4 & 2.03 & 1.43 & 4.36 & 8.85 \\
Sycon sp. (73) & 2.3 & 3.48 & 1.97 & 1.8 & 4.22 & 13.08 \\
Ecionemia c.f. acervus (8) & 2.08 & 2.42 & 1.7 & 1.04 & 3.65 & 16.72 \\
Haliclona sp. (6) & 1.11 & 1.3 & 1.52 & 1.37 & 3.25 & 19.97 \\
Chalinula milnei (4) & 1 & 0.54 & 1.46 & 1.04 & 3.13 & 23.1 \\
Protosuberites sp. (1) & 3.5 & 3.54 & 1.36 & 1.25 & 2.92 & 26.02 \\
Haplosclerina Sub order sp. (20) & 1.55 & 1.46 & 1.34 & 1.18 & 2.87 & 28.89 \\
Dysidea sp. 17 (17) & 1.73 & 1.64 & 1.33 & 1.21 & 2.86 & 31.75 \\
Tetilidae sp. (11) & 0.19 & 0.88 & 1.31 & 1.38 & 2.81 & 34.56 \\
Dysidea sp. 2328 (9) & 0.4 & 0.6 & 1.25 & 0.82 & 2.69 & 37.24 \\
Hymedesmia sp. (12) & 0.47 & 0.54 & 1.21 & 0.8 & 2.59 & 39.83 \\
Jaspis sp. (3) & 1.03 & 0.83 & 1.11 & 1.1 & 2.38 & 42.21 \\
Pericharax sp. (10) & 2.16 & 2.36 & 1.04 & 0.99 & 2.24 & 44.45 \\
Aaptos sp. (2) & 0.62 & 0.63 & 1 & 1.19 & 2.14 & 46.59 \\
Chelonaplysilla sp. (5) & 1.37 & 1.65 & 0.95 & 1.21 & 2.03 & 48.62 \\
Dendrilla sp. (15) & 0.69 & 0.96 & 0.94 & 1.14 & 2.01 & 50.63 \\
Callyspongia sp. (45) & 0.26 & 0.64 & 0.91 & 1.08 & 1.96 & 52.58 \\
Clathrina sp. (24) & 0.43 & 0.45 & 0.9 & 1.19 & 1.92 & 54.51 \\
Clathrina sp. (74) & 0 & 0.52 & 0.8 & 1.12 & 1.72 & 56.23 \\
Cream cushion (98) & 0 & 0.55 & 0.77 & 0.63 & 1.66 & 57.89 \\
Placospongia melobesioides(35) & 0.39 & 0.38 & 0.76 & 0.87 & 1.63 & 59.52 \\
Niphates sp.(19) & 0.44 & 0.26 & 0.72 & 0.87 & 1.54 & 61.06 \\
\hline Table 6.13 Species
\end{tabular}

Table 6.13 Species explaining the differences between all sites in 2005 and 2008. Average percentage difference between 2005 and 2008 was 52.31\% 
Chapter 6 Temporal and spatial diversity patterns of sponge assemblages in the Wakatobi Marine National Park, Indonesia.

\begin{tabular}{|l|c|c|c|c|c|c|}
\hline & $\begin{array}{c}2005 \text { Av. } \\
\text { Abund }\end{array}$ & $\begin{array}{c}\text { 2008 Av. } \\
\text { Abund }\end{array}$ & $\begin{array}{c}\text { Av. } \\
\text { Diss }\end{array}$ & $\begin{array}{c}\text { Diss/ } \\
\text { SD }\end{array}$ & $\begin{array}{c}\text { Contrib } \\
\%\end{array}$ & Cum.\% \\
\hline Sycon sp. (73) & 0.09 & 3.13 & 4.62 & 2.64 & 8.84 & 8.84 \\
Clathrina sp. (84) & 0 & 1.79 & 2.81 & 1.48 & 5.38 & 14.21 \\
Dysidea sp. 2328 (9) & 0.7 & 1.77 & 2.23 & 1.54 & 4.26 & 18.47 \\
Ecionemia c.f. acervus (8) & 2.87 & 2.02 & 1.96 & 1.05 & 3.75 & 22.21 \\
Petrosia (Petrosia) sp. (13) & 1.95 & 1.3 & 1.88 & 1.16 & 3.59 & 25.81 \\
Purple thin encrust. (16) & 1.16 & 0.12 & 1.83 & 1.64 & 3.49 & 29.3 \\
Haliclona sp. (6) & 1.65 & 1.32 & 1.67 & 1.22 & 3.19 & 32.49 \\
Protosuberites sp. (1) & 3.26 & 3.15 & 1.61 & 1.38 & 3.08 & 35.57 \\
Pericharax sp. (10) & 2.15 & 2.78 & 1.61 & 1.39 & 3.07 & 38.64 \\
Haplosclerina Sub order sp. (20) & 1.12 & 1.86 & 1.5 & 1.56 & 2.86 & 41.5 \\
Dysidea sp. 17 (17) & 1.57 & 1.66 & 1.45 & 1.1 & 2.78 & 44.28 \\
Jaspis sp. (3) & 1.05 & 0.88 & 1.29 & 1.15 & 2.46 & 46.74 \\
Dendrilla sp. (15) & 0.82 & 0.76 & 1.26 & 1.25 & 2.4 & 49.14 \\
Tedania sp. (123) & 0 & 0.86 & 1.16 & 0.69 & 2.21 & 51.35 \\
Tetilidae sp. (11) & 0.28 & 0.69 & 1.08 & 1.09 & 2.07 & 53.43 \\
Chalinula milnei (4) & 0.87 & 0.53 & 1.07 & 1.01 & 2.05 & 55.48 \\
Hymedesmia sp. (12) & 0.54 & 0.33 & 0.97 & 0.84 & 1.86 & 57.34 \\
Clathrina sp. (24) & 0.59 & 0.19 & 0.95 & 1.05 & 1.82 & 59.16 \\
Aaptos sp. (2) & 0.53 & 0.61 & 0.89 & 1.08 & 1.7 & 60.86 \\
\hline
\end{tabular}

Table 6.14 Species explaining the differences between all sites in 2006 and 2008. Average percentage difference between 2006 and 2008 was $47.11 \%$

\begin{tabular}{|l|c|c|c|c|c|c|}
\hline Species & $\begin{array}{c}\text { 2006 Av. } \\
\text { Abund }\end{array}$ & $\begin{array}{c}\text { 2008 Av. } \\
\text { Abund }\end{array}$ & $\begin{array}{c}\text { Av. } \\
\text { Diss }\end{array}$ & Diss/SD & $\begin{array}{c}\text { Contrib } \\
\%\end{array}$ & Cum.\% \\
\hline Clathrina sp. (84) & 0 & 1.79 & 2.84 & 1.47 & 6.02 & 6.02 \\
Dysidea sp. 2328 (9) & 0.4 & 1.77 & 2.65 & 1.61 & 5.62 & 11.65 \\
Sycon sp. (73) & 2.3 & 3.13 & 1.88 & 1.52 & 3.99 & 15.63 \\
Petrosia (Petrosia) sp. (13) & 1.54 & 1.3 & 1.81 & 1.41 & 3.85 & 19.48 \\
Ecionemia c.f. acervus (8) & 2.08 & 2.02 & 1.8 & 1.14 & 3.82 & 23.31 \\
Protosuberites sp. (1) & 3.5 & 3.15 & 1.54 & 1.38 & 3.28 & 26.58 \\
Chalinula milnei (4) & 1 & 0.53 & 1.45 & 0.95 & 3.07 & 29.66 \\
Haliclona sp. (6) & 1.11 & 1.32 & 1.38 & 1.25 & 2.94 & 32.59 \\
Dysidea sp. 17 (17) & 1.73 & 1.66 & 1.35 & 1.38 & 2.86 & 35.45 \\
Pericharax sp. (10) & 2.16 & 2.78 & 1.21 & 1.15 & 2.57 & 38.02 \\
Haplosclerina Sub order sp. (20) & 1.55 & 1.86 & 1.18 & 1.13 & 2.5 & 40.52 \\
Tedania sp. (123) & 0 & 0.86 & 1.17 & 0.69 & 2.48 & 43.01 \\
Jaspis sp. (3) & 1.03 & 0.88 & 1.09 & 1.02 & 2.32 & 45.33 \\
Chelonaplysilla sp. (5) & 1.37 & 1.66 & 1.06 & 1.29 & 2.25 & 47.58 \\
Tetilidae sp. (11) & 0.19 & 0.69 & 1.05 & 1.17 & 2.23 & 49.81 \\
Dendrilla sp. (15) & 0.69 & 0.76 & 1.01 & 1.2 & 2.13 & 51.94 \\
Hymedesmia sp. (12) & 0.47 & 0.33 & 0.92 & 0.74 & 1.96 & 53.9 \\
Aaptos sp. (2) & 0.62 & 0.61 & 0.9 & 1.14 & 1.91 & 55.81 \\
Grey translucent sp. (58) & 0.23 & 0.46 & 0.82 & 0.94 & 1.74 & 57.55 \\
Placospongia melobesioides (35) & 0.39 & 0.42 & 0.81 & 0.85 & 1.72 & 59.27 \\
Cribrochalina sp. (7) & 0.3 & 0.41 & 0.8 & 0.87 & 1.7 & 60.97 \\
\hline
\end{tabular}


Chapter 6 Temporal and spatial diversity patterns of sponge assemblages in the Wakatobi Marine National Park, Indonesia.

Table 6.15 Species explaining the differences between all sites in 2007 and 2008. Average percentage difference between 2007 and 2008 was $43.06 \%$

\begin{tabular}{|l|c|c|c|c|c|c|}
\hline Species & $\begin{array}{c}2007 \text { Av. } \\
\text { Abund }\end{array}$ & $\begin{array}{c}2008 \text { Av. } \\
\text { Abund }\end{array}$ & $\begin{array}{c}\text { Av. } \\
\text { Diss }\end{array}$ & $\begin{array}{c}\text { Diss/ } \\
\text { SD }\end{array}$ & $\begin{array}{c}\text { Contrib } \\
\%\end{array}$ & $\begin{array}{c}\text { Cum. } \\
\%\end{array}$ \\
\hline Protosuberites sp. (1) & 6.44 & 5.3 & 2.65 & 1.38 & 6.53 & 6.53 \\
Dysidea sp. 2328 (9) & 0.82 & 2.39 & 1.89 & 1.3 & 4.67 & 11.2 \\
Ecionemia c.f. acervus (8) & 3.51 & 2.97 & 1.69 & 1.14 & 4.18 & 15.38 \\
Sycon sp. (73) & 5.98 & 5.33 & 1.66 & 1.34 & 4.1 & 19.48 \\
Clathrina sp. (84) & 1.88 & 2.44 & 1.55 & 1.2 & 3.84 & 23.32 \\
Petrosia (Petrosia) sp. (13) & 0.49 & 1.74 & 1.27 & 0.98 & 3.14 & 26.46 \\
Haliclona sp. (6) & 1.74 & 1.83 & 1.21 & 1.25 & 2.98 & 29.43 \\
Pericharax sp. (10) & 3.19 & 4.03 & 1.19 & 1.29 & 2.93 & 32.36 \\
Dysidea sp. 17 (17) & 2.16 & 2.26 & 1.18 & 1.3 & 2.92 & 35.28 \\
Haplosclerina Sub order sp. (20) & 1.89 & 2.41 & 1.13 & 1.38 & 2.8 & 38.08 \\
Tedania sp. (123) & 0 & 1.19 & 0.94 & 0.68 & 2.32 & 40.4 \\
Hymedesmia sp. (12) & 0.72 & 0.44 & 0.87 & 0.75 & 2.15 & 42.54 \\
Jaspis sp. (3) & 1.05 & 1.13 & 0.82 & 0.93 & 2.03 & 44.57 \\
Aaptos sp. (2) & 0.81 & 0.81 & 0.75 & 1.17 & 1.85 & 46.42 \\
Tetilidae sp. (11) & 1.14 & 0.88 & 0.75 & 1.08 & 1.85 & 48.27 \\
Dendrilla sp. (15) & 1.25 & 1 & 0.74 & 1.22 & 1.83 & 50.1 \\
Chalinula milnei (4) & 0.69 & 0.67 & 0.69 & 0.96 & 1.7 & 51.79 \\
Purple thin encrust. sp. (16) & 0.62 & 0.15 & 0.68 & 0.86 & 1.68 & 53.47 \\
Callyspongia sp. (45) & 0.82 & 0.66 & 0.67 & 1.02 & 1.66 & 55.13 \\
Clathrina sp. (74) & 0.71 & 0.09 & 0.67 & 1.18 & 1.64 & 56.77 \\
Cream cushion sp. (98) & 0.73 & 0 & 0.59 & 0.64 & 1.45 & 58.22 \\
Placospongia melobesioides(35) & 0.5 & 0.55 & 0.58 & 0.96 & 1.42 & 59.65 \\
Cream encrust. (87) & 0.53 & 0.62 & 0.55 & 0.9 & 1.36 & 61.01 \\
\hline
\end{tabular}

Table 6.16 Species explaining the differences between all sites in 2005 and 2009. Average percentage difference between 2005 and 2009 was 59.31\%

\begin{tabular}{|l|c|c|c|c|c|c|}
\hline & $\begin{array}{c}2005 \text { Av. } \\
\text { Abund }\end{array}$ & $\begin{array}{c}2009 \text { Av. } \\
\text { Abund }\end{array}$ & $\begin{array}{c}\text { Av. } \\
\text { Diss }\end{array}$ & $\begin{array}{c}\text { Diss/ } \\
\text { SD }\end{array}$ & $\begin{array}{c}\text { Contrib } \\
\%\end{array}$ & $\begin{array}{c}\text { Cum. } \\
\%\end{array}$ \\
\hline Species & 0.09 & 2.21 & 3.24 & 1.49 & 5.47 & 5.47 \\
Ecionemia c.f. acervus (8) & 2.87 & 1.64 & 2.5 & 1.6 & 4.21 & 9.68 \\
Protosuberites sp. (1) & 3.26 & 2.34 & 2.37 & 1.34 & 3.99 & 13.67 \\
Petrosia (Petrosia) sp. (13) & 1.95 & 0.67 & 2.35 & 1.5 & 3.96 & 17.63 \\
Dysidea sp. 2328 (9) & 0.7 & 1.68 & 1.82 & 1.23 & 3.07 & 20.7 \\
Haplosclerina Sub order sp. (20) & 1.12 & 2.29 & 1.82 & 1.42 & 3.07 & 23.77 \\
Purple thin encrust. sp. (16) & 1.16 & 0.05 & 1.77 & 1.48 & 2.98 & 26.75 \\
Pericharax sp. (10) & 2.15 & 1.87 & 1.73 & 1.01 & 2.91 & 29.66 \\
Haliclona sp. (6) & 1.65 & 1.37 & 1.64 & 1.21 & 2.76 & 32.41 \\
Chelonaplysilla sp. (5) & 1.51 & 1.17 & 1.5 & 1.08 & 2.52 & 34.94 \\
Tedania sp. (123) & 0 & 1.04 & 1.43 & 0.69 & 2.4 & 37.34 \\
Hymedesmia sp. (12) & 0.54 & 0.93 & 1.42 & 1.21 & 2.39 & 39.73 \\
Dysidea sp. 17 (17) & 1.57 & 1.45 & 1.33 & 1.13 & 2.24 & 41.97 \\
Jaspis sp. (3) & 1.05 & 0.74 & 1.32 & 1.27 & 2.23 & 44.21 \\
Dendrilla sp. (15) & 0.82 & 0.42 & 1.32 & 1.08 & 2.23 & 46.43 \\
Clathrina sp. (84) & 0 & 0.86 & 1.32 & 0.69 & 2.22 & 48.65 \\
Chalinula milnei (4) & 0.87 & 0.34 & 1.2 & 0.99 & 2.02 & 50.67 \\
Aaptos sp. (2) & 0.53 & 0.72 & 1.07 & 1.33 & 1.8 & 52.46 \\
Callyspongia sp. (45) & 0.24 & 0.84 & 1.06 & 1.26 & 1.78 & 54.25 \\
Tetilidae sp. (11) & 0.28 & 0.51 & 0.99 & 0.9 & 1.68 & 55.92 \\
Clathria (Microcionia) lizardensis (41) & 0.07 & 0.65 & 0.95 & 0.77 & 1.6 & 57.53 \\
Clathrina sp. (24) & 0.59 & 0.07 & 0.94 & 1 & 1.58 & 59.1 \\
Cliona sp. (109) & 0 & 0.55 & 0.87 & 0.68 & 1.47 & 60.58 \\
\hline
\end{tabular}


Chapter 6 Temporal and spatial diversity patterns of sponge assemblages in the Wakatobi Marine National Park, Indonesia.

Table 6.17 Species explaining the differences between all sites in 2006 and 2009. Average percentage difference between 2006 and 2009 was $55.22 \%$

\begin{tabular}{|l|c|c|c|c|c|c|}
\hline Species & $\begin{array}{c}2006 \text { Av. } \\
\text { Abund }\end{array}$ & $\begin{array}{c}2009 \text { Av. } \\
\text { Abund }\end{array}$ & $\begin{array}{c}\text { Av. } \\
\text { Diss }\end{array}$ & $\begin{array}{c}\text { Diss/ } \\
\text { SD }\end{array}$ & $\begin{array}{c}\text { Contrib } \\
\%\end{array}$ & $\begin{array}{c}\text { Cum. } \\
\%\end{array}$ \\
\hline Protosuberites sp. (1) & 3.5 & 2.34 & 2.52 & 1.37 & 4.56 & 4.56 \\
Dysidea sp. 2328 (9) & 0.4 & 1.68 & 2.37 & 1.62 & 4.29 & 8.84 \\
Ecionemia c.f. acervus (8) & 2.08 & 1.64 & 2.18 & 1.45 & 3.94 & 12.78 \\
Petrosia (Petrosia) sp. (13) & 1.54 & 0.67 & 1.99 & 1.48 & 3.61 & 16.39 \\
Sycon sp. (73) & 2.3 & 2.21 & 1.93 & 1.29 & 3.49 & 19.88 \\
Haliclona sp. (6) & 1.11 & 1.37 & 1.6 & 1.38 & 2.9 & 22.78 \\
Hymedesmia sp. (12) & 0.47 & 0.93 & 1.54 & 1.21 & 2.78 & 25.56 \\
Haplosclerina Sub order sp. (20) & 1.55 & 2.29 & 1.48 & 1.2 & 2.68 & 28.24 \\
Chalinula milnei (4) & 1 & 0.34 & 1.46 & 0.97 & 2.64 & 30.88 \\
Tedania sp. (123) & 0 & 1.04 & 1.44 & 0.69 & 2.61 & 33.49 \\
Chelonaplysilla sp. (5) & 1.37 & 1.17 & 1.43 & 1.29 & 2.59 & 36.08 \\
Pericharax sp. (10) & 2.16 & 1.87 & 1.37 & 0.95 & 2.48 & 38.56 \\
Dendrilla sp. (15) & 0.69 & 0.42 & 1.35 & 1.38 & 2.44 & 41 \\
Clathrina sp. (84) & 0 & 0.86 & 1.32 & 0.69 & 2.39 & 43.39 \\
Dysidea sp. 17 (17) & 1.73 & 1.45 & 1.24 & 1.19 & 2.24 & 45.63 \\
Jaspis sp. (3) & 1.03 & 0.74 & 1.12 & 1.09 & 2.02 & 47.65 \\
Callyspongia sp. (45) & 0.26 & 0.84 & 1.06 & 1.29 & 1.92 & 49.57 \\
Clathria (Microcionia) lizardensis (41) & 0.15 & 0.65 & 1.02 & 0.81 & 1.84 & 51.42 \\
Aaptos sp. (2) & 0.62 & 0.72 & 1.02 & 1.28 & 1.84 & 53.25 \\
Pseudoceratina sp. (63) & 0.26 & 0.53 & 0.93 & 0.77 & 1.68 & 54.93 \\
Tetilidae sp. (11) & 0.19 & 0.51 & 0.91 & 0.96 & 1.65 & 56.59 \\
Grey translucent sp. (58) & 0.23 & 0.36 & 0.91 & 0.81 & 1.64 & 58.23 \\
Cliona sp. (109) & 0 & 0.55 & 0.88 & 0.68 & 1.6 & 59.83 \\
Axinyssa sp. (22) & 0.09 & 0.5 & 0.77 & 0.85 & 1.4 & 61.23 \\
\hline
\end{tabular}

Table 6.18 Species explaining the differences between all sites in 2007 and 2009. Average percentage difference between 2007 and 2009 was 53.29\%

\begin{tabular}{|l|c|c|c|c|c|c|}
\hline & $\begin{array}{c}\text { 2007 Av. } \\
\text { Abund }\end{array}$ & $\begin{array}{c}2009 \text { Av. } \\
\text { Abund }\end{array}$ & $\begin{array}{c}\text { Av. } \\
\text { Diss }\end{array}$ & $\begin{array}{c}\text { Diss/ } \\
\text { SD }\end{array}$ & $\begin{array}{c}\text { Contrib } \\
\%\end{array}$ & $\begin{array}{c}\text { Cum. } \\
\%\end{array}$ \\
Species & 3.54 & 2.34 & 2.36 & 1.48 & 4.43 & 4.43 \\
\hline Protosuberites sp. (1) & 1.34 & 0.86 & 2.26 & 1.31 & 4.24 & 8.67 \\
Clathrina sp. (84) & 3.48 & 2.21 & 2.12 & 1.02 & 3.98 & 12.65 \\
Sycon sp. (73) & 0.6 & 1.68 & 2.08 & 1.45 & 3.9 & 16.55 \\
Dysidea sp. 2328 (9) & 2.42 & 1.64 & 2.04 & 1.42 & 3.83 & 20.38 \\
Ecionemia c.f. acervus (8) & 2.36 & 1.87 & 1.48 & 1.06 & 2.78 & 23.16 \\
Pericharax sp. (10) & 0.54 & 0.93 & 1.45 & 1.2 & 2.72 & 25.88 \\
Hymedesmia sp. (12) & 1.3 & 1.37 & 1.44 & 1.22 & 2.71 & 28.59 \\
Haliclona sp. (6) & 1.46 & 2.29 & 1.43 & 1.25 & 2.68 & 31.27 \\
Haplosclerina Sub order sp. (20) & 1.65 & 1.17 & 1.31 & 1.07 & 2.47 & 33.73 \\
Chelonaplysilla sp. (5) & 0.96 & 0.42 & 1.31 & 1.9 & 2.46 & 36.19 \\
Dendrilla sp. (15) & 0 & 1.04 & 1.3 & 0.69 & 2.45 & 38.64 \\
Tedania sp. (123) & 1.64 & 1.45 & 1.27 & 1.32 & 2.38 & 41.02 \\
Dysidea sp. 17 (17) & 0.88 & 0.51 & 1.04 & 1.19 & 1.95 & 42.97 \\
Tetilidae sp. (11) & 0.83 & 0.74 & 1.01 & 1.02 & 1.9 & 44.87 \\
Jaspis sp. (3) & 0.4 & 0.67 & 0.98 & 0.9 & 1.84 & 46.7 \\
Petrosia (Petrosia) sp. (13) & 0.63 & 0.72 & 0.95 & 1.22 & 1.79 & 48.49 \\
Aaptos sp. (2) & 0.54 & 0.34 & 0.86 & 0.87 & 1.61 & 50.1 \\
Chalinula milnei (4) & 0.38 & 0.53 & 0.84 & 0.76 & 1.59 & 51.69 \\
Pseudoceratina sp. (63) & 0.64 & 0.84 & 0.83 & 1.05 & 1.55 & 53.24 \\
Callyspongia sp. (45) & 0.23 & 0.5 & 0.82 & 0.94 & 1.54 & 54.78 \\
Axinyssa sp. (22) & 0.55 & 0.12 & 0.82 & 0.71 & 1.54 & 56.32 \\
Cream cushion (98) & 0 & 0.55 & 0.79 & 0.68 & 1.49 & 57.81 \\
Cliona sp. (109) & 0.52 & 0.31 & 0.79 & 1.11 & 1.48 & 59.28 \\
Clathrina sp. (74)
\end{tabular}


Chapter 6 Temporal and spatial diversity patterns of sponge assemblages in the Wakatobi Marine National Park, Indonesia.

Table 6.19 Species explaining the differences between all sites in 2008 and 2009. Average percentage difference between 2008 and 2009 was $47.88 \%$

\begin{tabular}{|c|c|c|c|c|c|c|}
\hline Species & $\begin{array}{l}2008 \mathrm{Av} . \\
\text { Abund }\end{array}$ & $\begin{array}{l}2009 \text { Av. } \\
\text { Abund }\end{array}$ & $\begin{array}{l}\text { Av.Di } \\
\text { ss }\end{array}$ & $\begin{array}{c}\text { Diss/ } \\
\text { SD }\end{array}$ & $\begin{array}{c}\text { Contrib } \\
\%\end{array}$ & $\begin{array}{c}\text { Cum. } \\
\%\end{array}$ \\
\hline Clathrina sp. (84) & 1.79 & 0.86 & 2.19 & 1.2 & 4.57 & 4.57 \\
\hline Protosuberites sp. (1) & 3.15 & 2.34 & 2.15 & 1.45 & 4.48 & 9.05 \\
\hline Sycon sp. (73) & 3.13 & 2.21 & 1.93 & 0.97 & 4.02 & 13.08 \\
\hline Ecionemia c.f. acervus (8) & 2.02 & 1.64 & 1.86 & 1.35 & 3.88 & 16.96 \\
\hline Pericharax sp. (10) & 2.78 & 1.87 & 1.64 & 1.11 & 3.44 & 20.39 \\
\hline Dysidea sp. 2328 (9) & 1.77 & 1.68 & 1.5 & 1.2 & 3.14 & 23.53 \\
\hline Haliclona sp. (6) & 1.32 & 1.37 & 1.44 & 1.27 & 3.01 & 26.54 \\
\hline Petrosia (Petrosia) sp. (13) & 1.3 & 0.67 & 1.4 & 1.05 & 2.93 & 29.47 \\
\hline Chelonaplysilla sp. (5) & 1.66 & 1.17 & 1.36 & 1.04 & 2.84 & 32.31 \\
\hline Hymedesmia sp. (12) & 0.33 & 0.93 & 1.34 & 1.18 & 2.8 & 35.11 \\
\hline Dendrilla sp. (15) & 0.76 & 0.42 & 1.21 & 1.3 & 2.52 & 37.62 \\
\hline Dysidea sp. 17 (17) & 1.66 & 1.45 & 1.03 & 1.11 & 2.16 & 39.78 \\
\hline Jaspis sp. (3) & 0.88 & 0.74 & 0.96 & 1.13 & 2 & 41.79 \\
\hline Callyspongia sp. (45) & 0.51 & 0.84 & 0.96 & 1.19 & 2 & 43.78 \\
\hline Tedania sp. (123) & 0.86 & 1.04 & 0.94 & 0.63 & 1.95 & 45.73 \\
\hline Haplosclerina Sub order sp.(20) & 1.86 & 2.29 & 0.92 & 1.26 & 1.93 & 47.67 \\
\hline Aaptos sp. (2) & 0.61 & 0.72 & 0.92 & 1.26 & 1.92 & 49.58 \\
\hline Tetilidae sp. (11) & 0.69 & 0.51 & 0.89 & 1.09 & 1.86 & 51.44 \\
\hline Cliona sp. (109) & 0 & 0.55 & 0.84 & 0.67 & 1.75 & 53.19 \\
\hline Chalinula milnei (4) & 0.53 & 0.34 & 0.83 & 0.85 & 1.74 & 54.93 \\
\hline Pseudoceratina sp. (63) & 0.28 & 0.53 & 0.8 & 0.74 & 1.68 & 56.6 \\
\hline Grey translucent sp. (58) & 0.46 & 0.36 & 0.79 & 0.86 & 1.65 & 58.26 \\
\hline $\begin{array}{l}\text { Axinvssa sp. (22) } \\
\text { Clathria (Microcionia) lizardensis (41) }\end{array}$ & $\begin{array}{l}0.18 \\
0.33\end{array}$ & $\begin{array}{l}0.5 \\
0.65\end{array}$ & $\begin{array}{l}0.74 \\
0.71\end{array}$ & $\begin{array}{l}0.82 \\
0.72\end{array}$ & $\begin{array}{l}1.54 \\
1.49\end{array}$ & $\begin{array}{l}59.79 \\
61.28\end{array}$ \\
\hline
\end{tabular}

Table 6.20 Species explaining the similarity across all years in site A. Average similar for site A was $61.00 \%$

\begin{tabular}{|l|c|c|c|c|c|}
\hline Species & Av.Abund & Av.Sim & Sim/SD & Contrib\% & Cum.\% \\
\hline Protosuberites sp. (1) & 3.79 & 10.69 & 4.44 & 17.52 & 17.52 \\
Ecionemia c.f. acervus (8) & 2.71 & 6.94 & 2.74 & 11.39 & 28.9 \\
Sycon sp. (73) & 2.53 & 6.24 & 1.39 & 10.23 & 39.14 \\
Pericharax sp. (10) & 2.19 & 5.75 & 2.78 & 9.43 & 48.57 \\
Haplosclerina Sub order sp. (20) & 1.96 & 5.22 & 3.18 & 8.57 & 57.13 \\
Dysidea sp. 17 (17) & 1.86 & 4.81 & 2.41 & 7.89 & 65.02 \\
\hline
\end{tabular}

Table 6.21 Species explaining the similarity across all years in site B. Average similar for site B was $48.79 \%$

\begin{tabular}{|l|c|c|c|c|c|}
\hline Species & Av.Abund & Av.Sim & Sim/SD & Contrib\% & Cum.\% \\
\hline Protosuberites sp. (1) & 2.92 & 8.03 & 2.74 & 16.46 & 16.46 \\
Pericharax sp. (10) & 2.02 & 4.82 & 1.22 & 9.89 & 26.35 \\
Chelonaplysilla sp. (5) & 1.68 & 4.54 & 2.44 & 9.3 & 35.65 \\
Haplosclerina Sub order sp. (20) & 1.55 & 3.97 & 2.06 & 8.14 & 43.8 \\
Sycon sp. (73) & 1.49 & 3.49 & 1.01 & 7.15 & 50.95 \\
Ecionemia c.f. acervus (8) & 1.54 & 3.11 & 0.77 & 6.38 & 57.33 \\
Dysidea sp. 2328 (9) & 1.29 & 2.81 & 0.85 & 5.76 & 63.08 \\
\hline
\end{tabular}


Chapter 6 Temporal and spatial diversity patterns of sponge assemblages in the Wakatobi Marine National Park, Indonesia.

Table 6.22 Species explaining the similarity across all years in site $\mathrm{C}$. Average similar for site $\mathrm{C}$ was $56.43 \%$

\begin{tabular}{|l|c|c|c|c|c|}
\hline Species & Av.Abund & Av.Sim & Sim/SD & Contrib\% & Cum.\% \\
\hline Sycon sp. (73) & 2.71 & 6.69 & 1.9 & 11.86 & 11.86 \\
Pericharax sp. (10) & 2.58 & 6.32 & 4.82 & 11.19 & 23.05 \\
Protosuberites sp. (1) & 2.77 & 5.36 & 1.57 & 9.5 & 32.55 \\
Ecionemia c.f. acervus (8) & 2.37 & 5.24 & 1.58 & 9.28 & 41.84 \\
Dysidea sp. 17 (17) & 2.13 & 4.44 & 2.78 & 7.87 & 49.71 \\
Haliclona sp. (6) & 1.99 & 3.91 & 2.21 & 6.94 & 56.64 \\
Petrosia (Petrosia) sp. (13) & 1.42 & 2.59 & 1 & 4.6 & 61.24 \\
\hline
\end{tabular}

Table 6.23 Species explaining the differences between sites A and B across all years. Average percentage difference between sites A and B was $50.39 \%$

\begin{tabular}{|l|c|c|c|c|c|c|}
\hline & $\begin{array}{c}\text { A Av. } \\
\text { Abund }\end{array}$ & $\begin{array}{c}\text { B Av. } \\
\text { Abund }\end{array}$ & $\begin{array}{c}\text { Av. } \\
\text { Diss }\end{array}$ & $\begin{array}{c}\text { Diss/ } \\
\text { SD }\end{array}$ & $\begin{array}{c}\text { Contrib } \\
\%\end{array}$ & Cum.\% \\
\hline Ecionemia c.f. acervus (8) & 2.71 & 1.54 & 2.35 & 1.18 & 4.66 & 4.66 \\
Dysidea sp. 17 (17) & 1.86 & 0.83 & 2.01 & 1.47 & 3.98 & 8.64 \\
Sycon sp. (73) & 2.53 & 1.49 & 1.93 & 1.12 & 3.83 & 12.47 \\
Protosuberites sp. (1) & 3.79 & 2.92 & 1.83 & 1.29 & 3.64 & 16.1 \\
Dysidea sp. 2328 (9) & 0.68 & 1.29 & 1.67 & 1.09 & 3.31 & 19.41 \\
Haliclona sp. (6) & 1.12 & 0.94 & 1.64 & 1.3 & 3.25 & 22.67 \\
Petrosia (Petrosia) sp. (13) & 1.25 & 0.84 & 1.64 & 1.38 & 3.25 & 25.92 \\
Pericharax sp. (10) & 2.19 & 2.02 & 1.63 & 1.23 & 3.23 & 29.15 \\
Clathrina sp. (84) & 0.61 & 1.23 & 1.61 & 0.82 & 3.2 & 32.35 \\
Hymedesmia sp. (12) & 0.74 & 0.44 & 1.36 & 0.95 & 2.7 & 35.05 \\
Jaspis sp. (3) & 1.22 & 1.06 & 1.35 & 1.33 & 2.68 & 37.73 \\
Haplosclerina Sub order sp. (20) & 1.96 & 1.55 & 1.28 & 1.28 & 2.54 & 40.27 \\
Cream encrust. coral (87) & 0.2 & 0.81 & 1.2 & 1.27 & 2.38 & 42.65 \\
Chelonaplysilla sp. (5) & 1.77 & 1.68 & 1.15 & 1.18 & 2.29 & 44.94 \\
Chalinula milnei (4) & 0.34 & 0.57 & 1.13 & 0.83 & 2.25 & 47.19 \\
Dendrilla sp. (15) & 0.71 & 0.52 & 0.99 & 1.15 & 1.96 & 49.14 \\
Aaptos sp. (2) & 0.59 & 0.47 & 0.98 & 1.19 & 1.95 & 51.1 \\
Clathria (Microcionia) lizardensis (41) & 0.16 & 0.66 & 0.97 & 0.86 & 1.92 & 53.02 \\
Cribrochalina sp. (7) & 0.24 & 0.53 & 0.92 & 1.01 & 1.83 & 54.84 \\
Purple thin encrust. sp. (16) & 0.59 & 0.16 & 0.92 & 0.84 & 1.83 & 56.67 \\
Tetilidae sp. (11) & 0.49 & 0.41 & 0.88 & 0.81 & 1.74 & 58.41 \\
Neopetrosia sp. (31) & 0.06 & 0.49 & 0.85 & 0.82 & 1.68 & 60.09 \\
\hline
\end{tabular}


Chapter 6 Temporal and spatial diversity patterns of sponge assemblages in the Wakatobi Marine National Park, Indonesia.

Table 6.24 Species explaining the differences between sites $A$ and $C$ across all years. Average percentage difference between sites $A$ and $C$ was $46.50 \%$

\begin{tabular}{|l|c|c|c|c|c|c|}
\hline & A Av. & C Av. & Av. & Diss/ & Contrib & \\
Species & Abund & Abund & Diss & SD & $\%$ & Cum.\% \\
\hline Protosuberites sp. (1) & 3.79 & 2.77 & 2.22 & 1.4 & 4.78 & 4.78 \\
Haliclona sp. (6) & 1.12 & 1.99 & 1.81 & 1.57 & 3.89 & 8.67 \\
Petrosia (Petrosia) sp. (13) & 1.25 & 1.42 & 1.59 & 1.36 & 3.41 & 12.08 \\
Chelonaplysilla sp. (5) & 1.77 & 0.97 & 1.58 & 1.58 & 3.41 & 15.49 \\
Dysidea sp. 2328 (9) & 0.68 & 1.12 & 1.47 & 1.12 & 3.17 & 18.66 \\
Ecionemia c.f. acervus (8) & 2.71 & 2.37 & 1.42 & 1.03 & 3.06 & 21.72 \\
Jaspis sp. (3) & 1.22 & 0.43 & 1.42 & 1.68 & 3.05 & 24.76 \\
Chalinula milnei (4) & 0.34 & 1.06 & 1.34 & 1.21 & 2.88 & 27.64 \\
Haplosclerina Sub order sp. (20) & 1.96 & 1.46 & 1.33 & 1.18 & 2.86 & 30.5 \\
Hymedesmia sp. (12) & 0.74 & 0.51 & 1.3 & 1.04 & 2.8 & 33.3 \\
Dysidea sp. 17 (17) & 1.86 & 2.13 & 1.2 & 1.14 & 2.57 & 35.88 \\
Tedania sp. (123) & 0.19 & 0.95 & 1.13 & 0.62 & 2.43 & 38.3 \\
Dendrilla sp. (15) & 0.71 & 0.95 & 1.12 & 0.98 & 2.42 & 40.72 \\
Clathrina sp. (84) & 0.61 & 0.55 & 1.12 & 0.78 & 2.41 & 43.12 \\
Sycon sp. (73) & 2.53 & 2.71 & 1.02 & 0.84 & 2.19 & 45.32 \\
Placospongia melobesioides (35) & 0.14 & 0.66 & 1.02 & 0.92 & 2.19 & 47.5 \\
Pericharax sp. (10) & 0.4 & 0.84 & 1.01 & 1.09 & 2.17 & 49.67 \\
Pseudoceratina sp. (63) & 0.59 & 0.81 & 1 & 1.29 & 2.14 & 51.81 \\
Tetilidae sp. (11) & 0 & 0.66 & 0.99 & 0.76 & 2.13 & 53.94 \\
Grey translucent sp. (58) & 2.19 & 2.58 & 0.91 & 1.04 & 1.96 & 55.9 \\
\hline
\end{tabular}

Table 6.25 Species explaining the differences between sites $B$ and $C$ across all years. Average percentage difference between sites $B$ and $C$ was $55.19 \%$

\begin{tabular}{|l|c|c|c|c|c|c|}
\hline & B Av. & C Av. & Av. & Diss/ & Contrib & \\
Species & Abund & Abund & Diss & SD & $\%$ & Cum.\% \\
\hline Dysidea sp. 17 (17) & 0.83 & 2.13 & 2.17 & 1.43 & 3.93 & 3.93 \\
Haliclona sp. (6) & 0.94 & 1.99 & 1.94 & 1.43 & 3.52 & 7.44 \\
Protosuberites sp. (1) & 2.92 & 2.77 & 1.92 & 1.4 & 3.49 & 10.93 \\
Ecionemia c.f. acervus (8) & 1.54 & 2.37 & 1.9 & 1.16 & 3.44 & 14.38 \\
Sycon sp. (73) & 1.49 & 2.71 & 1.75 & 1.05 & 3.17 & 17.55 \\
Pericharax sp. (10) & 2.02 & 2.58 & 1.54 & 1.22 & 2.8 & 20.35 \\
Chalinula milnei (4) & 0.57 & 1.06 & 1.51 & 1.25 & 2.74 & 23.09 \\
Petrosia (Petrosia) sp. (13) & 0.84 & 1.42 & 1.45 & 1.03 & 2.63 & 25.72 \\
Haplosclerina Sub order sp. (20) & 1.55 & 1.46 & 1.45 & 1.33 & 2.62 & 28.34 \\
Jaspis sp. (3) & 1.06 & 0.43 & 1.4 & 1.2 & 2.53 & 30.88 \\
Chelonaplysilla sp. (5) & 1.68 & 0.97 & 1.4 & 1.52 & 2.53 & 33.41 \\
Dendrilla sp. (15) & 0.52 & 0.95 & 1.32 & 1.09 & 2.39 & 35.8 \\
Dysidea sp. 2328 (9) & 1.29 & 1.12 & 1.32 & 1.07 & 2.38 & 38.18 \\
Tedania sp. (123) & 0 & 0.95 & 1.27 & 0.67 & 2.3 & 40.49 \\
Cream encrust. sp. (87) & 0.81 & 0.07 & 1.15 & 1.25 & 2.08 & 42.57 \\
Aaptos sp. (2) & 0.47 & 0.81 & 1.08 & 1.33 & 1.96 & 44.53 \\
Clathrina sp. (84) & 1.23 & 0.55 & 1.07 & 0.76 & 1.94 & 46.47 \\
Placospongia melobesioides (35) & 0.4 & 0.66 & 1.05 & 1.05 & 1.89 & 48.37 \\
Callyspongia sp. (45) & 0.25 & 0.84 & 1.04 & 1.07 & 1.89 & 50.25 \\
Chalinula sp. (50) & 0 & 0.66 & 1.01 & 0.76 & 1.83 & 52.08 \\
Hymedesmia sp. (12) & 0.44 & 0.51 & 0.94 & 0.79 & 1.69 & 53.78 \\
Cinachyrella sp. (67) & 0 & 0.59 & 0.92 & 1.13 & 1.67 & 55.45 \\
Clathria (Microcionia) lizardensis (41) & 0.66 & 0 & 0.92 & 0.89 & 1.66 & 57.11 \\
Pseudoceratina sp. (63) & 0.11 & 0.62 & 0.83 & 0.79 & 1.51 & 58.62 \\
Tetilidae sp. (11) & 0.41 & 0.63 & 0.8 & 0.94 & 1.44 & 60.06 \\
\hline
\end{tabular}


Chapter 6 Temporal and spatial diversity patterns of sponge assemblages in the Wakatobi Marine National Park, Indonesia. 
CHAPTER 7

\section{General DisCussion}


Understanding temporal and spatial variation in biodiversity patterns is one of the most fundamental problems in ecology (Gaston, 2000; Tittensor et al., 2010). Most research has focused on elucidating spatial patterns of biodiversity (Soest, 1994; Bellwood \& Hughes, 2001; Cruz-Motta et al., 2010), however, there has been increasing interest in trying to understand the role of temporal dynamics to understand assemblage patterns over seasonal to geological timescales (Sheppard, 1995; Aronson, 2001; Axis-Arroya \& Mateu, 2004; Willis et al., 2007; Boakes et al., 2010).

The primary goals of this thesis were to understand the spatial and temporal pattern of sponge assemblage variation over a variety of scales. Understanding the natural variability of sponge assemblages may help develop suitable monitoring methods and allow sponges to be incorporated into marine habitat management plans. This is important because sponges are an ecologically significant group in benthic marine communities (Sara, 2004; McClintock et al., 2005; Becerro, 2008) and yet they are often ignored in monitoring schemes (Rützler, 1978; Wulff, 2001). This discussion will examine the consistencies in temporal and spatial patterns of sponges from the locations studied in this thesis; this could help with designing monitoring schemes for sponges and potentially other marine benthos.

One of the key findings from chapter 2 was that approximately $50 \%$ of the spatial variability of known sponge biodiversity in New Zealand waters can be explained by the geographical position (Latitude:Longitude) and depth and genus level data is a good surrogate for species-level data. There were however, large gaps in the data set, such as the west coast of the South Island and also limited data available away from the main urban centres and marine biological research stations. Therefore it is now important to explore the most efficient way to try and fill those gaps in the sponge biodiversity dataset and these methods could be applied to other un-surveyed areas. 
Ideally, a thorough biodiversity survey should be conducted across a range of habitats, evenly spaced sites, and a range of depths by sponge taxonomists along the coast. However, this is unlikely to happen because there is a shortage of taxonomists in New Zealand (and worldwide) for all phyla, including sponges (Bradford-Grieve, 2008), and an intensive biodiversity survey would be expensive and time consuming. A method that was successful in this thesis (chapter 2) and in other regional biodiversity surveys is to collect and use presence genera level data (Hooper et al., 2002; Terlizzi et al., 2003; Terlizzi et al., 2009). The advantages are that it is quicker and easier to identify species to genera level than species level (especially as many species are still to be described); which therefore reduces the cost which can then be used towards carrying out more spatial surveys (Balmford et al., 1996). Presence absence/data of genera or families has also been found to reflect the regional patterns for sponges in the Mediterranean (Voultsiadou, 2009; Xavier, 2009), Indonesia (de Voogd \& Cleary, 2008) and for other marine taxa such as algae (Kerswell, 2006), molluscs (Terlizzi et al., 2009) and fish (Tolimieri \& Anderson, 2010).

A randomly selected list of at least ten species/genera has been suggested as sufficient basic information needed in order to predict the richness of biodiversity of an area using taxonomic distinctness measures (Rowden et al., 2004). However, for sponges, care should be taken to record more genera if possible as one of the sites, Lyttelton Port, which was specious within the genera Haliclona (12 out of the 26 species recorded), had only ten genera recorded and had significantly different biodiversity values for taxonomic distinctness for the species and genera data. Therefore, although the taxonomic distinctness indices are largely independent of sample size, there can be a small bias in the results leading to an underestimate of their values with very small species lists (Clarke \& Warwick, 1998; Pienkowski et al., 1998; Clarke \& Warwick, 2001b).

Once broad scale surveys have identified areas of high sponge biodiversity they can provide a priority list of where taxonomists are needed to work up the 
samples from biodiversity hotspots. Areas with values of AvTD and/or VarTD above the values predicted for the $95 \%$ probability funnel have significantly more diverse sponge assemblages then the surrounding region and therefore should be prioritised as containing habitats which need to be included in habitat management and monitoring plans (Fig. 7.1).

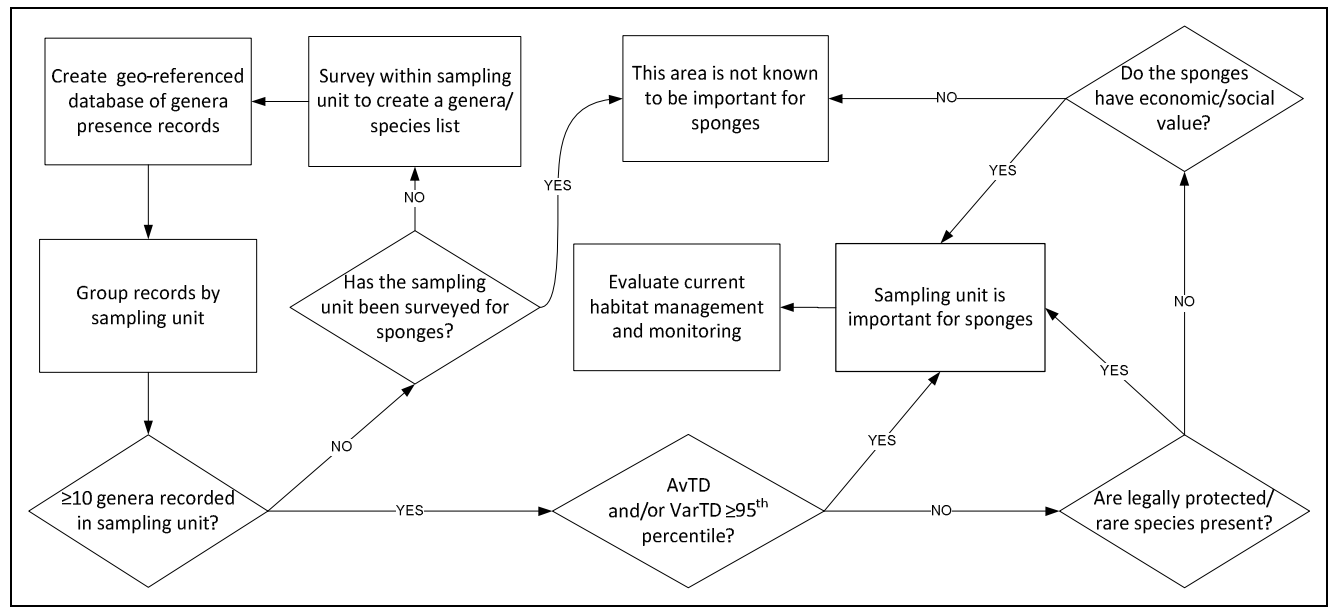

Fig. 7.1 A flowchart (starting at the top left corner) to assist in identifying sampling areas that are sponge biodiversity hotspots and therefore need to be incorporated into habitat management and monitoring schemes.

One of the areas found to have significantly higher levels of AvTD and VarTD was the Wellington South Coast, a location where the spatial variation was examined in Chapter 3 and temporally in Chapter 4. The sponge densities on the Wellington South Coast were variable but can reach up to 500 sponges $\mathrm{m}^{-2}$ and $50 \%$ percentage coverage. There were no significant differences in species richness $\mathrm{m}^{-2}$ between geographical locations on the Wellington South coast, however, there were significant seasonal differences and a significant interaction of site and the season. Site, season and the interaction factor season*site were also important factors for explaining significant differences in the total abundance and the multivariate data on the Wellington South Coast. The same factors were also significant for explaining the variability of the Skomer sponges assemblage structure in chapter 5 (although there were no significant differences in the species richness values across the three sites, years and seasons). There has been little published research integrating the combined effect of spatial and temporal effects on assemblages (but see White et al., 
2010). Most studies concentrate on either spatial or temporal variation, and don't take into account the effects of the potential interaction between spatiotemporal factors. Pure spatial variation shows a gradient of abundance and/or diversity in space and there are no changes over time. Pure temporal variation has shifts in abundance and/or diversity over time however there are no differences due to spatial gradients. If spatial and temporal variations change independently of each other, then both patterns can be seen across all sites and time. In the case of both Wellington and Skomer assemblages there was clear spatiotemporal variation, where changes over time and space lead to a shifting mosaic of assemblage structure between sites and over time.

A given sampling unit (for example a quadrat) within a habitat may contain a number of core species, which are present throughout all the seasons and, occasional species (which may or may not already be present within the wider habitat) that have immigrated/emigrated into/out of the quadrat. The area surrounding a quadrat will have different species available for immigration. Therefore quadrats in heterogeneous areas, with a more diverse regional species pool, will include a greater total number of species over time compared with quadrats in a more homogeneous region even if they have similar values for species richness at a given time point. Because core species by definition have viable populations, there is likely to be low temporal turnover in species composition among the core species (Magurran \& Henderson, 2003). By contrast, occasional species are frequently recruited from adjacent habitats and are ephemeral, resulting in high values of temporal turnover.

Sponges are thought to have limited larval dispersal abilities due to the brief period of their larval planktonic stage and limited swimming and/or crawling ability (Maldonado, 2006; Mariani et al., 2006). Asexual reproduction, such as the budding of Tethya spp. or the fission (and fusion) of encrusting species, appears to play a structuring role at small spatial scales as the buds/patches remain close to the original adult sponge (Corriero et al., 1996) (Blanquer et al., 2009). However, in chapter 2 , all of the twenty most common genera recorded across New Zealand were found across wide geographical and depth ranges (0- 
200m), and some genera, such as Haliclona and Clathrina (species of both these genera were found at all the study sites in this thesis), have a worldwide distribution (Soest, 1994). Biogeography patterns of sponges do not differ greatly from other marine groups and therefore distributions are in general determined by large-scale geographical factors, such as tectonic events, and physical factors such as differences in sea temperature, continental run-off and deep sea barriers (Soest, 1994). Potential long-range dispersal methods, include rafting on other biota (Barnes \& Fraser, 2003) or catching a ride either in ballast water or attached to ships as invasive species, are often found first in harbours (Coles et al., 1997; Hutchings et al., 2002). There are some sponge species, such as Clathrina clathrus, which were thought to be cosmopolitan, however, genetic studies have revealed that there are actually two or more cryptic species identified under the same species name and the ranges of 'cosmopolitan' species are often more restricted than previously thought (SoléCava et al., 1991). It is important to consider that sponge species can be cryptic within the same habitat and sponges which appear visually identical may have differing ecological strategies such as growth or reproduction during differing seasons and therefore have differing functional roles within the habitat (Blanquer et al., 2008).

The main limitations of including sponges within monitoring schemes and conservation management plans is the limit of taxonomic knowledge and funding for taxonomic work here in New Zealand and globally (Wilson, 2004; Bradford-Grieve, 2008). Underestimates of global sponge diversity and other sessile taxa have resulted primarily from a lack of comprehensive localityspecific sampling and difficulties in classification (Hooper et al., 2002; Manconi \& Pronzato, 2008; Lanna et al., 2009; Xavier, 2009).

The World Porifera database (van Soest et al., 2008) provides a searchable list of sponge species that is regularly updated with the latest nomenclature changes and links to the relevant literature for further identification information. The sponge barcoding project has been developed in order to create a set of tools to aid in the rapid identification of sponge species, in 
particular in relation to the discovery of drug-producing species. (Wörheide \& Erpenbeck, 2007). Barcoding is already revealing new species and leading to explanations of sponge biogeography (Pöppe et al., 2010). Barcoding is unlikely to replace taxonomy completely as the boundaries of where a species begins and ends within a genus are not easily defined, and the database of molecular codes need to be backed up with ecological and morphological information, however, it is a useful tool to compliment other conservation management methods (Schindel \& Miller, 2005).

Additive diversity partitioning is a method which has recently been used for conservation management in order to understand the distribution of biodiversity across spatial (Gering et al., 2003; Summerville et al., 2003; Belmaker et al., 2008; Zvuloni et al., 2010) and temporal scales (Summerville \& Crist, 2005; Wu et al., 2010). The results from Indonesia (chapter 6) showed that although the sponge assemblages were changing in the actual species present and their abundances (using multivariate methods), the proportion of diversity within each spatial level (quadrat, site and region) remained consistent when sampled at the same time each year throughout the five year study. In species rich assemblages there are a variety of life strategies that can respond differently to shifts in environmental conditions and contribute to ecological functioning in various ways, however, there is also a high level of functional redundancy, which means that there are multiple species which can carry out the same functional roles increasing the overall stability of the assemblage (McCann, 2000; Tilman et al., 2006; Yachi \& Loreau, 2006). The abundances of each species required to carry out the same role within an ecosystem are not necessarily the same, therefore abundances of species can fluctuate in a system with functional redundancy and the ecosystem can remain stable. Functional diversity is an important component of biodiversity for understanding the ecosystem processes and the extent of functional differences within a community (Petchey \& Gaston, 2002). It is often measured by counting the number of functional groups represented by a species within the community (Tilman et al., 2001). There is likely to be a high level of functional redundancy within sponge assemblages (Bell, 2007a), which may explain why 
the diversity within the quadrats, sites and local area remain within the same proportions. A high level of biodiversity does not always equate to ecological functional redundancy (Magurran \& Henderson, 2010). For example, reversal of phase shifts (e.g. from coral to macroalgal dominance) can be driven by species with 'sleeping' functional roles (i.e., a species or group of species capable of performing a particular functional role but which does so only under exceptional circumstances) (Bellwood et al., 2006; Cheal et al., 2010).

Due to the difficulty in reliably identifying sponges, morphological monitoring methods have been proposed as a surrogate in order to represent the functional diversity present and get an approximation for the patterns of biodiversity (Bell \& Barnes, 2001, 2002; Bell et al., 2006; Bell, 2007a, b). Morphological groups correlated significantly ( $\rho=0.01 \%$ ) with the species data in Skomer Marine Reserve (Chapter 5). The collection of species-level data is approximately forty times more expensive than collecting morphological data due to extended in water time, laboratory time and the need for specialist taxonomic skills (Bell et al., 2006). However, species level surveys are important in order to identify species at the edge of their range, that are endemic, rare or those that are potentially economically valuable for biological compounds. Morphological data should be collected simultaneously in order to see if the patterns in morphology correlate with the species assemblage shifts as for some habitats this is not the case, for example, in caves and under boulders (Bell \& Barnes, 2001).

Various monitoring methods have been tested using sponge assemblages over various spatial and temporal scales in this thesis. Spatial, temporal and spatiotemporal interaction terms were all important for distinguishing significant assemblage differences in all the localities and further studies integrating spatio-temporal data sets are vital to understand the potential responses of ecological systems to global change and provide scientifically sound management plans (White et al., 2010). Taxonomic distinctness measures are useful for initially assessing how the biodiversity is distributed, especially when using a data set with uneven sampling, as it is robust to spatial and temporal bias (Clarke \& Warwick, 1998, 2001b). Therefore the taxonomic distinctness measures are especially useful for historical datasets, which are a 
useful source of data but are often biased spatially and temporally (Willis et al., 2007; Sloan \& Bartier, 2009; Boakes et al., 2010). Broad-scale monitoring programmes cannot effectively detect trends in all species at all spatial scales therefore a toolkit is needed with a variety of methods for monitoring on local scales (Nielsen et al., 2009). A comprehensive biodiversity survey preferably at species level with samples collected for genetic analysis is still the best first step in surveying a new site. A biodiversity survey provides not only a vital baseline for future monitoring but also will identify species that could have potential economic and pharmaceutical potential. In order to know if an assemblage is changing then the abundance of species present collected simultaneously with morphological data will assess that the viability of using morphological monitoring for that site. If they correlate significantly then morphological monitoring can be used as a surrogate for the functional diversity. If the morphological data do not correlate with the species data other potential methods to save time and money include monitoring only the key species driving the changes in the assemblages, but care must be taken as the key species may change over time and new species not in the original survey may become important.

All of the sponge assemblages monitored had significant levels of variability in abundance and diversity between quadrats both within and between sites. Therefore high levels of variability are normal with the Skomer, Wellington and Indonesian sponge assemblages, suggesting this could be similar for other sponge rich habitats. All the sites showing significant yet not always predictable annual shifts where there is a high turnover in species and abundance which could mean they all have high levels of functional redundancy similar to the results from Indonesia. Water temperature was a key driver of change in the assemblages however there was not always a consistent pattern within or between sites with a higher temperature leading to increase in the numbers of sponges present at one site and decline in another. Therefore micro variations in spatial factors such as the depth, aspect and substratum and the current assemblage present is likely to interact with environmental factors such as sea temperature which will shift the balance to favour slightly different species in 
different sites. The monitoring methods compared within this thesis hopefully provide a suite of tools for integrating sponges into marine monitoring plans and provide the right methods for a variety of ecological questions from understanding how diversity in partitioned within an area to understanding the biogeography of sponges using Taxonomic Distinctness measures. The time period and spatial scale necessary to detect a real change at a specified level needs to be provided to people planning habitat management to ensure the success of biodiversity monitoring programmes and that the most robust indicators of biodiversity are selected. 
REFERENCES 
Abdo, D.A., McDonald, J.I., Harvey, E.S., Fromont, J. \& Kendrick, G.A. (2008) Neighbour and environmental influences on the growth patterns of two temperate Haliclonid sponges. Marine and Freshwater Research, 59, 304-312

Abdo, D.A., Motti, C.A., Battershill, C.N. \& S., H.E. (2007) Temperature and spatiotemporal variability of Salicylihalamide A in the sponge Haliclona sp. Journal of Chemical Ecology, 33, 1635-1645

Ackers, R.G., Moss, D., Picton, B.E., Stone, S.M.K. \& Morrow, C.C. (2007) Sponges of the British Isles (Sponge V), 1992 reset with modifications, 2007 edn. Marine Conservation Society, Belfast.

Adjeroud, M. \& Salvat, B. (1996) Spatial patterns in biodiversity of a fringing-reef community along Opunohu Bay, Moorea, French Polynesia. Bulletin of Marine Science, 59, 175-187

Aerts, L.M.A. (1998) Sponge/coral interactions in Caribbean reefs: analysis of overgrowth patterns in relation to species identity and cover. Marine Ecology Progress Series, 175, 241-249.

Akaike, H. (1987) Factor analysis and AIC. Psychometrika, 52, 317-332

Alcolado, P.M. (1979) Nueva especie deporifero (género Strongylophora) encontrada en Cuba. POEYANA, 196, 1-5

Alcolado, P.M. (1990) General features of Cuban sponge communities. New Perspectives in Sponge Biology (ed. by K. Rützler), pp. 351-357. Smithsonian Institution Press, Washington, D.C.

Alcolado, P.M. (1994) General trends in coral reef sponge communities of Cuba. Sponges in time and space; Biology, Chemistry, Paleontology (ed. by R.W.M.V. Soest, T.G. Van Kempen and J.C. Braekman), pp. 251-255. A.A. Balkema, Rotterdam.

Allan, J.D. (1975) Components of diversity. Oecologia, 18, 359-367

Allen, G.R. (2008) Conservation hotspots of biodiversity and endemism for Indo-Pacific coral reef fishes. Aquatic Conservation: Marine and Freshwater Ecosystems, 18, 541-556

Allison, G.W., Lubchenco, J. \& Carr, M.H. (1998) Marine reserves are necessary but not sufficient for marine conservation. . Ecological Applications, 8, S79-S92

Alvarez, B., Diaz, M.C. \& Laughlin, R.A. (1990) The sponge fauna on a fringing coral reef in Venezuela, I: composition, distribution and abundance. New perspectives in sponge biology (ed. by R. K), pp. 358-366. Smithsonian Institute Press, London.

Amano, S. (1990) Self and non-self recognition in a calcareous sponge, Leucandra abratsbo. Biological Bulletin, 179, 272-278

Amir, I. (1992) A comparison of sponge fauna of exposed and sheltered reef flats in eastern Indonesia. Marine Research Indonesia, 28, 1-12

Anderson, M.J. (2001) A new method for non-parametric multivariate analysis of variance. Austral Ecology, 26, 32-46

Anon (2000) The New Zealand Biodiversity Strategy. Department of Conservation and Ministry for the Environment, Wellington Available at http://www.biodiversity.govt.nz/index.html Available at: (accessed 25-March 2011).

Anon (2005) The New Zealand Marine Environment Classification. Ref. ME594 Department of Conservation and Ministry for the Environment, Wellington Available at http://www.mfe.govt.nz/publications/ser/marine-environment-classification-jun05/ (accessed 25-March 2011).

Anon (2010) NBN Gateway. Available at http://data.nbn.org.uk/gridSquares/10kmSquareSpeciesList.jsp?parentSq=SM70\&allDs=1\&desi 
References

g=0\&eng0rd=0\&src=1\&pg=1\&spGrpKey=116\&mapService=tenKmSelectorGBv4\&homePageY=2 92\&homePageX=103 (accessed 25-March 2011).

Apte, S. \& Gardner, J.P.A. (2002) Population genetic subdivision in the New Zealand greenshell mussel (Perna canaliculus) inferred from single-strand conformation polymorphism analysis of mitochondrial DNA. Molecular Ecology, 11, 1617-1628

Araújo, M.B., Humphries, C.J., Densham, P.J., Lampinen, R., Hagemeijer, W.J.M., Mitchell-Jones, A.J. \& Gasc, J.P. (2001) Would environmental diversity be a good surrogate for species diversity? Ecography, 24, 103-110

Arillo, A., Bavestrello, G., Burlando, B. \& Sara, M. (1993) Metabolic integration between symbiotic cyanobacteria and sponges: a possible mechanism. Marine Biology, 117, 159-162

Arndt, W. (1935) Porifera. Die Tierwelt der Nord und Ostsee (ed. by G. Grimpe and E. Wagler), p. 140. Grimpe \& Wagler, Leipzig.

Aronson, R.B. (2001) The limits of detectability: short-term events and short-distance variation in the community structure of coral reefs. Bulletin of Marine Science, 69, 331-332

Avila, C. \& Paul, V.J. (1997) Chemical ecology of the nudibranch Glossodoris pallida: is the location of dietderived metabolites important for defense? Marine Ecology Progress Series, 150, 171-180

Ávila, E. \& Carballo, J. (2008) A preliminary assessment of the invasiveness of the Indo-Pacific sponge Chalinula nematifera on coral communities from the tropical Eastern Pacific. Biological Invasions,

Axis-Arroya, J. \& Mateu, J. (2004) Spatio-temporal modeling of benthic biological species. Journal of Environmental Management, 71, 67-77.

Ayers, K.L. \& Waters, J.M. (2005) Marine biogeographic disjunction in central New Zealand. Marine Biology, 147, 1045-1052

Ayling, A.L. (1979) Subtidal sponges in the Cape Rodney to Okakari Point marine reserve. In, p. 84. University of Auckland Marine Laboratory

Ayling, A.L. (1980) Patterns of Sexuality, Asexual Reproduction and Recruitment in Some Subtidal Marine Demospongiae. Biological Bulletin, 158, 271-282

Ayling, A.L. (1981) The Role of Biological Disturbance in Temperate Subtidal Encrusting Communities. Ecology, 62, 830-847

Ayling, A.L. (1983a) Factors affecting the spatial distributions of thinly encrusting sponges from temperate waters. Oecologia, 60, 412-418.

Ayling, A.L. (1983b) Growth and regeneration rates in thinly encrusting Demospongiae from temperate waters. Biological Bulletin, 165, 343-352

Bachraty, C., Legendre, P. \& Desbruyères, D. (2009) Biogeographic relationships among deep-sea hydrothermal vent faunas at global scale. Deep Sea Research Part I: Oceanographic Research Papers, 56, 1371-1378

Baco, A.R., Rowden, A.A., Levin, L.A., Smith, C.R. \& Bowden, D.A. (2010) Initial characterization of cold seep faunal communities on the New Zealand Hikurangi margin. Marine Geology, 272, 251-259

Balmford, A. \& Gaston, K.J. (1999) Why biodiversity surveys are good value. Nature, 398, 204-205

Balmford, A., Green, M.J.B. \& Murray, M.G. (1996) Using higher-taxon richness as a surrogate for species richness .1. Regional tests. Proceedings of the Royal Society of London Series B-Biological Sciences, 263, 1267-1274

Balsamo Crivelli, G. (1863) Di alcuni Spongiari del Golfo di Napoli. Atti della Societa Italiana di Scienze Naturali e del Museo Civico di Storia Naturale di Milano, 5, 284-302 
References

Bannister, R.J., Battershill, C.N. \& Nys, R.d. (2010) Demographic variability and long-term change in a coral reef sponge along a cross-shelf gradient of the Great Barrier Reef. Marine and Freshwater Research, 61, 389-396

Barnes, D.K.A. (1999) High diversity of tropical intertidal zone sponges in temperature, salinity and current extremes. African Journal of Ecology, 37, 424-434

Barnes, D.K.A. \& Bell, J.J. (2002) Coastal sponge communities of the West Indian Ocean: Taxonomic affinities, richness and diversity. African Journal of Ecology, 40, 337-349.

Barnes, D.K.A. \& Fraser, K.P.P. (2003) Rafting by five phyla on man-made flotsam in the Southern Ocean. Marine Ecology Progress Series, 262, 289-291

Barnes, D.K.A. \& Kuklinski, P. (2004) Variability of competition at scales of $10^{1}, 10^{3}, 10^{5}$ and $10^{6} \mathrm{~m}$ : encrusting arctic community patterns. Marine Biology, 145, 361-372

Barthel, D. (1986) On the ecophysiology of the sponge Halichondria panicea in Kiel Bight. 1. Substrate specificity, growth and reproduction. Marine Ecology-Progess Series, 32, 291-298

Battershill, C.N. (1987) Factors affecting the structure and dynamics of subtidal communities characterised by sponges. In: Department of Zoology, p. 599. University of Auckland, Auckland

Battershill, C.N. \& Bergquist, P.R. (1990) Marine sponges: forty-six sponges of Northern New Zealand. In: Leigh Laboratory Bulletin, pp. 1-149. University of Auckland Marine Laboratory, Auckland

Battershill, C.N. \& Page, M.J. (1996) Preliminary Survey of Pariokariwa Reef North Taranaki. In, p. 15. New Zealand Oceanographic Institute, National Institute of Water and Atmospheric Research Ltd, NIWA, Wellington

Beaumont, J., MacDiarmid, A. \& d'Archino, R. (2010) Mapping the environmental values of New Zealand's ecosystem. A meta-analysis. Biosecurity NZ Technical Paper, 2010, 1-76

Beaumont, J., Oliver, M. \& Macdiarmid, A. (2008) Mapping the values of New Zealand's coastal waters. 1. Environmental values. Biosecurity NZ Technical Paper, 2008, 1-89

Becerro, M.A. (2008) Quantitative trends in sponge ecology research. Marine Ecology, 29, 167-177

Becerro, M.A., Paul, V.J. \& Starmer, J. (1998) Intracolonial variation in chemical defenses of the sponge Cacospongia sp. and its consequences on generalist fish predators and the specialist nudibranch predator Glossodoris pallida. Marine Ecology Progress Series, 168, 187-196

Becking, L.E., Cleary, D.F.R., Voogd, N.J., Renema, W., Beer, M., Soest, R.W.M. \& Hoeksema, B.W. (2006) Beta diversity of tropical marine benthic assemblages in the Spermonde Archipelago, Indonesia. Marine Ecology, 27, 76-88

Bell, J. \& Carballo, J. (2008) Patterns of sponge biodiversity and abundance across different biogeographic regions. Marine Biology, 155, 563-570

Bell, J.J. (2002) Regeneration rates of a temperate Demosponge: the importance of water flow rate. Journal of the Marine Biological Association of the United Kingdom, 82, 169-170

Bell, J.J. (2004) Evidence for morphology-induced sediment settlement prevention on the tubular sponge Haliclona urceolus. Marine Biology, 146, 29-38

Bell, J.J. (2007a) Contrasting patterns of species and functional composition of coral reef sponge assemblages. Marine Ecology Progress Series, 339, 73-81

Bell, J.J. (2007b) The use of volunteers for conducting sponge biodiversity assessments and monitoring using a morphological approach on Indo-Pacific coral reefs. Aquatic Conservation: Marine and Freshwater Ecosystems, 17, 133-145

Bell, J.J. (2008) The functional roles of marine sponges. Estuarine, Coastal and Shelf Science, 79, 341-353. 
References

Bell, J.J. \& Barnes, D.K.A. (2000a) A sponge diversity centre within a marine 'island'. Hydrobiologia, 440, 55-64

Bell, J.J. \& Barnes, D.K.A. (2000b) The distribution and prevalence of sponges in relation to environmental gradients within a temperate sea lough: Vertical cliff surfaces. Diversity and Distributions, 6, 283303

Bell, J.J. \& Barnes, D.K.A. (2000c) The distribution and prevalence of sponges in relation to environmental gradients within a temperate sea lough: Inclined cliff surfaces. Diversity and Distributions, 6, 305323

Bell, J.J. \& Barnes, D.K.A. (2001) Sponge morphological diversity: A qualitative predictor of species diversity? Aquatic Conservation: Marine and Freshwater Ecosystems, 11, 109-121

Bell, J.J. \& Barnes, D.K.A. (2002) Modelling sponge species diversity using a morphological predictor: A tropical test of a temperate model. Journal for Nature Conservation, 10, 41-50

Bell, J.J. \& Barnes, D.K.A. (2003a) Effect of disturbance on assemblages: an example using Porifera. Biological Bulletin, 205, 144-159

Bell, J.J. \& Barnes, D.K.A. (2003b) The importance of competitor identity, morphology and ranking methodology to outcomes in interference competition between sponges. Marine Biology, 143, 415-426

Bell, J.J. \& Smith, D. (2004) Ecology of sponge assemblages (Porifera) in the Wakatobi region, south-east Sulawesi, Indonesia: Richness and abundance. Journal of the Marine Biological Association of the United Kingdom, 84, 581-591

Bell, J.J., Berman, J., Jones, T. \& Hepburn, L.J. (2010) Variability in the spatial association patterns of sponge assemblages in response to environmental heterogeneity. Marine Biology (Berlin), 157, 25032509

Bell, J.J., Burton, M., Bullimore, B., Newman, P.B. \& Lock, K. (2006) Morphological monitoring of subtidal sponge assemblages. Marine Ecology Progress Series, 311, 79-91

Bellwood, D.R. \& Hughes, T.P. (2001) Regional-scale assembly rules and biodiversity of coral reefs. Science, 292, 1532-1535

Bellwood, D.R., Hughes, T.P. \& Hoey, A.S. (2006) Sleeping functional group drives coral-reef recovery. Current Biology, 16, 2434-2439

Belmaker, J., Ziv, Y., Shashah, N. \& Connolly, S.R. (2008) Regional variation in the hierarchical partitioning of diversity in coral-dwelling fishes. Ecology, 89, 2829-40

Belmaker, J., Ziv, Y., Shashah, N. \& Connolly, S.R. (2008) Regional variation in the hierarchical partitioning of diversity in coral-dwelling fishes. Ecology, 89, 2829-40

Benedetti-Cecchi, L., Airoldi, L., Abbiati, M. \& Cinelli, F. (1997) Exploring the causes of spatial variation in an assemblage of benthic invertebrates from a submarine cave with sulphur springs. Journal of Experimental Marine Biology and Ecology, 208, 153-168

Benedetti-Cecchi, L., Bertocci, I., Vaselli, S., Maggi, E. \& Bulleri, F. (2008) Neutrality and the response of rare species to environmental variance. PLoS ONE, 3, e2777

Bergquist, P.R. (1961) A collection of Porifera from northern New Zealand, with descriptions of seventeen new species. Pacific Science, 15, 33-48

Bergquist, P.R. (1968) The marine fauna of New Zealand: Porifera, Demospongiae, Part 1 (Tetractinomorpha and Lithistida). New Zealand Department of Scientific and Industrial Research Bulletin, 188, 1-105 
References

Bergquist, P.R. (1970) The marine fauna of New Zealand: Porifera, Demospongiae, Part 2 (Axinellida and Halichondrida). New Zealand Department of Scientific and Industrial Research Bulletin, 197, 1-85

Bergquist, P.R. (1996) The marine fauna of New Zealand: Porifera: Demospongiae Part 5. Dendroceratida and Halisarcida. New Zealand Oceanographic Institute Memoir, 107, 1-53

Bergquist, P.R. \& Fromont, P.J. (1988) The marine fauna of New Zealand: Porifera, Demospongiae, Part 4 (Poecilosclerida). New Zealand Oceangraphic Institute Memoir, 96, 1-197

Bergquist, P.R. \& Kelly-Borges, M. (1991) An evaluation of the genus Tethya (Porifera: Demospongiae: Hadromerida) with descriptions of new species from the southwest Pacific. The Beagle, Records of the Northern Territory Museum of Arts and Sciences, 8, 37-72

Bergquist, P.R. \& Sinclair, M.E. (1968) The morphology and behaviour of larvae of some intertidal sponges. New Zealand Journal of Marine and Freshwater Research, 2, 426-37

Bergquist, P.R. \& Tizard, C.A. (1967) Australian intertidal sponges from the Darwin area. Micronesica, 3, 175-202

Bergquist, P.R. \& Warne, K.P. (1980) The marine fauna of New Zealand: Porifera, Demospongiae, Part 3 (Haplosclerida and Nepheliospongida). New Zealand Oceangraphic Institute Memoir, 87, 1-77

Berman, J. \& Bell, J.J. (2010) Spatial variability of sponge assemblages on the Wellington South Coast, New Zealand. The Open Marine Biology Journal, 4, 12-25

Berman, J., Perea-Blazquez, A., Kelly, M. \& Bell, J.J. (2008) Sponges of the Wellington South Coast. The Taputeranga marine reserve (ed. by J.P.A. Gardner and J.J. Bell), pp. 225-236. First Edition Ltd., Wellington.

Beu, A. \& Marshall, B. (2008) Mollusca of the Wellington South Coast. The Taputeranga marine reserve (ed. by G.J.P. A. and J.J. Bell), pp. 262-. First Edition Ltd, Wellington.

Bibiloni, M.A., Uriz, M.J. \& Gili, J.M. (1989) Sponge communities in three submarine caves of the Balearic Islands (western Mediterranean): adaptations and faunistic composition. P.S.Z.N.I: Marine Ecology, 10, 317-334

Blanquer, A., Uriz, M. \& Caujapé-Castells, J. (2009) Small-scale spatial genetic structure in Scopalina lophyropoda, an encrusting sponge with philopatric larval dispersal and frequent fission and fusion events. Marine Ecology Progress Series, 380, 95-102

Blanquer, A., Uriz, M.J. \& Agell, G. (2008) Hidden diversity in sympatric sponges: Adjusting life-history dynamics to share substrate. Marine Ecology Progress Series, 371, 109-115

Boakes, E.H., McGowan, P.J.K., Fuller, R.A., Chang-qing, D., Clark, N.E., O'Connor, K. \& Mace, G.M. (2010) Distorted views of biodiversity: Spatial and temporal bias in species occurrence data. PLoS Biol, 8 , e1000385

Boero, F. (2010) The study of species in the era of biodiversity: a tale of stupidity. Diversity, 2, 115-126

Booth, J. (1975) Seasonal and tidal variations in the hydrology of Wellington Harbour. N.Z. Journal of Marine and Freshwater Research, 9, 333-354

Borcard, D., Legendre, P. \& Drapeau, P. (1992) Partialling out the spatial component of ecological variation. Ecology, 73, 1045-1055

Borg, J.A. \& Schembri, P.J. (1996) Preliminary data on the occurrence and distribution of shallow water marine sponges (Porifera) around Maltese coasts. Xjenza, 1, 24-28

Bouchet, P. (2006) The magnitude of marine biodiversity. The Exploration of Marine Biodiversity: Scientific and Technological Challenges (ed. by C.M. Duarte), pp. 31-64. Fundacion BBVA, Bilbao, Spain. 
References

Boury-Esnault, N., Pandini, M. \& Uriz, M.J. (1993) Cosmopolitism in sponges: The "complex" Guitarra fimbriata with description of a new species of Guitarra from the northeast Atlantic. Scientia Marina, 57, 367-373

Boury-Esnault, N., Solé-Cava, A.M. \& Thorpe, J.P. (1992) Genetic and cytological divergence between colour morphs of the Mediterranean sponge Oscarella lobularis Schmidt (Porifera, Demospongiae, Oscarellidae). Journal of Natural History, 26, 271-284

Bowerbank, J.S. (1864) A monograph of the British Spongiadae. The Ray Society, London.

Bowerbank, J.S. (1866) A monograph of the British Spongiadae. The Ray Society, London.

Bowerbank, J.S. (1874) A monograph of the British Spongiadae. The Ray Society, London.

Bowerbank, J.S. \& Norman, A.M. (1882) A monograph of the British Spongiadae. The Ray Society, London.

Bradford-Grieve, J. (2008) Absence of government leadership is damaging the health of systematics and taxonomy in the UK. New Zealand Science Review, 65, 84-88

Bryan, P.G. (1973) Growth rate, toxicity, and distribution of the encrusting sponge Terpios sp. (Hadromerida: Subertidae) in Guam, Marina Islands. Micronesica, 9, 237-242

Bullimore, B. (1986) Skomer marine reserve subtidal monitoring project. Hydrobiologica, 142, 340

Burlando, B., Bavestrello, G. \& Arillo, A. (1992) Seasonal changes in the metabolism of the calcareous sponge Clathrina clathrus (Schmidt). Comparative Biochemistry and Physiology, A, 101A, 341-344

Burnham, K.P. \& Anderson, D.R. (2002) Model selection and multi-model inference - A practical informationtheoretic approach, $2^{\text {nd }}$ edn. Springer-Verlag, New York.

Burton, M. (1949) Observations on littoral sponges, including the supposed swarming of larvae, movement and coalescence in mature individuals, longevity and death. Proceedings of the Zoological Society of London, 118, 893-915

Burton, M., Lock, K., Gibbs, R. \& Newman, P. (2007) Skomer marine nature reserve project status report 2006/07. In: CCW Regional Report, p. 75

Burton, M., Lock, K., Gibbs, R. \& Newman, P. (2008) Skomer Marine Nature Reserve Project Status Report 2007/8. In: CCW Regional Report, p. 95.

Butler, A.J. \& Chesson, P.L. (1990) Ecology of sessile animals on hard substrata: The need to measure variation. Aust J Ecol, 15, 521-531

Carballo, J.L. (2006) Effect of natural sedimentation on the structure of tropical sponge assemblages. Ecoscience, 13, 119-130.

Carballo, J.L. \& Nava, H. (2007) A comparison of sponge assemblage patterns in two adjacent rocky habitats (tropical Pacific Ocean, Mexico). Ecoscience, 14, 92-102

Carballo, J.L., Vega, C., Cruz-Barraza, J.A., Yanez, B., Nava, H., Avila, E. \& Wilson, M. (2008) Short- and longterm patterns of sponge diversity on a rocky tropical coast: evidence of large-scale structuring factors. Marine Ecology, 29, 216-236.

Carter, L. (2008) Below low tide - a seabed in motion. The Taputeranga marine reserve (ed. by J.P.A. Gardner and J.J. Bell), pp. 130-144. First Edition, Wellington.

Carter, L. \& Lewis, K. (1995) Variability of the modern sand cover in a tide and storm driven inner shelf, south Wellington, New Zealand. New Zealand Journal of Geology \& Geophysics, 38, 451-470.

Carter, L., Garlick, R.D., Sutton, P., Chiswell, S., Oien, N.A. \& Stanton, B.R. (1998) Ocean circulation New Zealand. NIWA chart. In. Miscellaneous Series 76., NIWA, Wellington, New Zealand

Carter, L., Laing, A. \& Bell, R. (2002) Wild southerlies of summer. In: Water and Atmosphere, pp. 22-23. NIWA, Wellington, New Zealand 
Cervino, J.M., Winiarski-Cervino, K., Polson, S.W., Goreau, T. \& Smith, G.W. (2006) Identification of bacteria associated with a disease affecting the marine sponge Ianthella basta in New Britain, Papua New Guinea. Marine Ecology Progress Series, 324, 139-150

Chandy, S., Gibson, D.J. \& Robertson, P.A. (2006) Additive partitioning of diversity across hierarchical spatial scales in a forested landscape. Journal of Applied Ecology, 43, 792-801

Chang, F.H., Zeldis, J., Gall, M. \& Hall, J. (2003) Seasonal and spatial variation of phytoplankton assemblages, biomass and cell size from spring to summer across the north-eastern New Zealand continental shelf. Journal of Plankton Research, 25, 737-758

Chao, A., Chazdon, R.L., Colwell, R.K. \& Shen, T.-J. (2005) A new statistical approach for assessing similarity of species composition with incidence and abundance data. Ecology Letters, 8, 148-159

Chapman, A.D. (2009) Numbers of living species in Australia and the World. In: Australian Biological Resources Study Canberra, Australia Available http://www.environment.gov.au/biodiversity/abrs/publications/other/speciesnumbers/index.html (accessed on 25/03/2011)

Chappell, J. (1980) Coral morphology, diversity and reef growth Nature, 286, 249-252

Cheal, A., MacNeil, M., Cripps, E., Emslie, M., Jonker, M., Schaffelke, B. \& Sweatman, H. (2010) Coralmacroalgal phase shifts or reef resilience: links with diversity and functional roles of herbivorous fishes on the Great Barrier Reef. Coral Reefs, 29, 1005-1015

Chen, X., Li, B.-L. \& Zhang, X.-S. (2008) Using spatial analysis to monitor tree diversity at a large scale: a case study in Northeast China Transect. Journal of Plant Ecology, 1, 137-141

Clark, M.R. \& O' Driscoll, R. (2003) Deepwater fisheries and aspects of their impact on seamount habitat in New Zealand. J Northw Atlantic Fish Sci, 31, 441-458

Clark, M.R., Rowden, A.A., Schlacher, T., Williams, A., Consalvey, M., Stocks, K.I., Rogers, A.D., O'Hara, T.D., White, M., Shank, T.M. \& Hall-Spencer, J.M. (2010) The Ecology of Seamounts: Structure, Function, and Human Impacts. Annual Review of Marine Science, 2, 253-278

Clarke, A. (2001) Benthic organisms and environmental variability in Antarctica: responses to seasonal, decadal and long term change. Ocean and Polar Research, 23, 433-440

Clarke, K. \& Gorley, R. (2006) PRIMER v6: User Manual/Tutorial. Plymouth

Clarke, K., Somerfield, P. \& Gorley, R. (2008) Testing of null hypotheses in exploratory community analyses: similarity profiles and biota-environment linkage. Journal of Experimental Marine Biology \& Ecology, 366, 56-69

Clarke, K.R. \& Warwick, R.M. (1998) A taxonomic distinctness index and its statistical properties. Journal of Applied Ecology, 35, 523-531

Clarke, K.R. \& Warwick, R.M. (2001a) Change in marine communities: an approach to statistical analysis and interpretation, 2nd edn. PRIMER-E, Plymouth.

Clarke, K.R. \& Warwick, R.M. (2001b) A further biodiversity index applicable to species lists: variation in taxonomic distinctness. Marine Ecology Progress Series, 216, 265-278

Clarke, K.R., Chapman, M.G., Somerfield, P.J. \& Needham, H.R. (2006) Dispersion-based weighting of species counts in assemblage analyses. Marine Ecology Progress Series, 320, 11-27

Clarke, K.R., Somerfield, P.J. \& Chapman, M.G. (2006) On resemblance measures for ecological studies, including taxonomic dissimilarities and a zero-adjusted Bray-Curtis coefficient for denuded assemblages. Journal of Experimental Marine Biology and Ecology, 330, 55-80

Cleary, D.F.R., Becking, L.E., de Voogd, N.J., Renema, W., de Beer, M., van Soest, R.W.M. \& Hoeksema, B.W. (2005) Variation in the diversity and composition of benthic taxa as a function of distance 
References

offshore, depth and exposure in the Spermonde Archipelago, Indonesia. Estuarine, Coastal and Shelf Science, 65, 557-570

Coles, S.L., de Felice, R.C., Eldredge, L.G. \& Carlton, J.T. (1997) Biodiversity of marine communities in Pearl Harbor, Oahu, Hawaii with observations on introduced marine species. Bishop Museum of Technology Reports, 10

Colwell, R.K. (2009) EstimateS: Statistical estimation of species richness and shared species from samples. Version 8.2. User's Guide and application. Available at: http://purl.oclc.org/estimates. (accessed 25/03/2011)

Commenges, D., Sayyareh, A., Letenneur, L., Guedj, J. \& Bar-Hen, A. (2008) Estimating a difference of Kullback-Leibler risks using a normalized difference of AIC. Annals of Applied Statistics, 2, 11231142

Contini, H. (1995) Growth rate and propagation of the Red Sea sponge Latrunculia magnifica: a study in situ and in vitro. Israel Journal of Zoology, 41, 82-83

Corredor, J.E., Wilkinson, C.R., Vicente, V.P., Morell, J.M. \& Otero, E. (1988) Nitrate release by Caribbean reef sponges. Limnological Oceanography, 33, 114-120.

Corriero, G. (1989) The sponge fauna from the Stagnone di Marsala (Sicily): Taxonomic and ecological observations. Bollettino dei Musei e degli Istituti Biologici della Universita di Genova, 53, 101-113

Corriero, G., Sara, M. \& Vaccaro, P. (1996) Sexual and asexual reproduction in two species of Tethya (Porifera, Demospongiae) from a Mediterranean coastal lagoon. Marine Biology, 126, 175-181

Costello, M.J., Coll, M., Danovaro, R., Halpin, P., Ojaveer, H. \& Miloslavich, P. (2010) A Census of Marine Biodiversity Knowledge, Resources, and Future Challenges. PLoS ONE, 5, e12110

Crabbe, J. \& Smith, D.J. (2002) Real-time monitoring of reef health and coral recruitment using digital videophotography and computer image analysis and modelling in the Wakatobi Marine National Park, S.E. Sulawesi, Indonesia. Coral Reefs, 21, 242-244

Cranfield, H.J., Gordon, D.P., Willan, R.C., Marshall, B.A., Battershill, C.N., Francis, M.P., Nelson, W.A., Glasby, C.J. \& Read, G.B. (1998) Adventive marine species in New Zealand. In, pp. 1-48. NIWA, Wellington

Cruz-Motta, J.J., Miloslavich, P., Palomo, G., Iken, K., Konar, B., Pohle, G., Trott, T., Benedetti-Cecchi, L., Herrera, C., Hernández, A., Sardi, A., Bueno, A., Castillo, J., Klein, E., Guerra-Castro, E., Gobin, J., Gómez, D.I., Riosmena-Rodríguez, R., Mead, A., Bigatti, G., Knowlton, A. \& Shirayama, Y. (2010) Patterns of spatial variation of assemblages associated with intertidal rocky shores: A global perspective. PLoS ONE, 5, e14354

Cryer, M., O'Shea, S., Gordon, D., Kelly, M., Dury, J., Morrison, M., Hill, A., Saunders, H., Shankar, U., Wilkinson, M. \& Foster, G. (2000) Distribution and structure of benthic communities between North Cape and Cape Reinga. In, p. 154. NIWA

da Costa, L.M., Keller, W.D. \& Johns, W.D. (1992) Espiculas de esponjas em solos de Joao Pinheiro, Minas Gerais. Revista, Ceres, 597-603

Davidson, R.J. (1991) A report on the intertidal and shallow subtidal ecology of the Abel Tasman National Park, Nelson. In, p. 161. Department of Conservation, Nelson/Marlborough Conservancy

Davidson, R.J. \& Brown, D.A. (1994) Ecological report on four marine reserve options: Eastern D'Urville Island area. In, p. 41. Department of Conservation, Nelson/Marlborough Conservancy

Dayton, P.K. (1978) Observations of growth, dispersal and population dynamics of some sponges in McMurdo sound, Antarctica. Colloques Intern C.N.R.S., 291, 271-282. 
References

Dayton, P.K. (1979) Observations of growth, dispersal and population dynamics of some sponges in McMurdo Sound, Antarctica. Biologie des Spongiaires - Sponge Biology (ed. by C. Lévi and N. Boury-Esnault), pp. 271-282. Editions du C.N.R.S., Paris.

Dayton, P.K. (1989) Interdecadal variation in an Antarctic sponge and its predators from oceanographic climate shifts. Science, 243, 1484-1486

Dayton, P.K. (2003) The importance of natural sciences to conservation. American Naturalist, 162, 1-13

de Caralt, S., Uriz, M.J. \& Wijffels, R.H. (2008) Grazing, differential size-class dynamics and survival of the Mediterranean sponge Corticium candelabrum. Marine Ecology Progress Series, 360, 97-106

de Goeij, J.M., De Kluijver, A., van Duyl, F.C., Vacelet, J., Wijffels, R.H., de Goeij, A.F.P.M., Cleutjens, J.P.M. \& Schutte, B. (2009) Cell kinetics of the marine sponge Halisarca caerulea reveal rapid cell turnover and shedding. J Exp Biol, 212, 3892-3900

de Lange, W., Bell, R.G., Gorman, R. \& Reid, S. (2003) Physical oceanography of New Zealand waters. The New Zealand Coast: Te Tai o Aotearoa (ed. by J. Goff, S. Nichol and H. Rouse), pp. 59-78. Dunmore Press, Palmerston North.

de Laubenfels, M.W.D. (1953) Sponges from the Gulf of Mexico. Bulletin of Marine Science of the Gulf and Caribbean, 2, 511-557

de Ronde, C.E.J., Baker, E.T., Massoth, G.J., Lupton, J.E., Wright, I.C., Sparks, R.J., Bannister, S.C., Reyners, M.E., Walker, S.L., Greene, R.R., Ishibashi, J., Faure, K., Resing, J.A. \& Lebon, G.T. (2007) Submarine hydrothermal activity along the mid-Kermadec Arc, New Zealand: Large-scale effects on venting. Geochemistry Geophysics Geosystems, 8, Q07007

de Voogd, N.J. \& Cleary, D.F.R. (2007) Relating species traits to environmental variables in Indonesian coral reef sponge assemblages. Marine and Freshwater Research, 58, 240-249

de Voogd, N.J. \& Cleary, D.F.R. (2008) An analysis of sponge diversity and distribution at three taxonomic levels in the Thousand Islands/Jakarta Bay reef complex, West-Java, Indonesia. Marine Ecologyan Evolutionary Perspective, 29, 205-215

de Voogd, N.J., Becking, L.E., Hoeksema, B.W., Noor, A. \& van Soest, R.W.M. (2004) Sponge interactions with spatial competitors in the Spermonde Archipelago. Bollettino dei Musei e degli Istituti Biologici della Universita di Genova, 68, 253-261.

de Voogd, N.J., Cleary, D.F.R., Hoeksema, B.W., Noor, A. \& Van Soest, R.W.M. (2006) Sponge beta diversity in the Spermonde Archipelago, SW Sulawesi, Indonesia. Marine Ecology Progress Series, 309, 131142

de Voogd, N.J., van Soest, R.W.M. \& Hoeksema, B.W. (1999) Cross-shelf distribution of southwest Sulawesi reef sponges. Memoirs of the Queensland Museum, 44, 147-154

de Vries, P.J., Murray, D. \& Lande, R. (1997) Species diversity in vertical, horizontal, and temporal dimensions of a fruit-feeding butterfly community in an Ecuadorian rainforest. Biological Journal of the Linnean Society, 62, 343-364

Dendy, A. (1914) Observations on the gametogenesis of Grantia compressa. Quarterly Journal of Microscopical Science, 60, 313-376

Diaz, C.M. \& Rützler, K. (2001) Sponges: An essential component of Caribbean coral reefs. Bulletin of Marine Science, 69, 535-546

Diaz, M.C., Alvarez, B. \& Laughlin, R.A. (1990) The sponge fauna on a fringing coral reef in Venezuela, II. Community structure. New Perspectives in Sponge Biology (ed. by K. Rützler), pp. 367-375. Smithsonian Institution Press, Washington, D.C. 
Diaz, M.C., Pomponi, S.A. \& van Soest, R.W.M. (1993) A systematic revision of the central West Atlantic: Halichondrida (Demospongiae, Porifera). Part III: Description of valid species. Scientia Marina, 57, 283-306

Dragnewitsch, P. (1906) Spongien von Singapore. Zoologische Jahrbücher, 23, 439-448

Duckworth, A.R. \& Battershill, C.N. (2001) Population dynamics and chemical ecology of New Zealand demospongiae Latrunculia sp. nov. and Polymastia croceus (Poecilosclerida: Latrunculiidae: Polymastiidae). New Zealand Journal of Marine and Freshwater Research, 35, 935-949

Duckworth, A.R., Battershill, C.N. \& Bergquist, P.R. (1997) Influence of explant procedures and environmental factors on culture success of three sponges. Aquaculture, 156, 251-267

Duckworth, A.R., Battershill, C.N. \& Schiel, D.R. (2004) Effects of depth and water flow on growth, survival and bioactivity of two temperate sponges cultured in different seasons. Aquaculture, 242, 237250

Duckworth, A.R., Wolff, C., Evans-Illidge, E., Whalan, S. \& Lui, S. (2008) Spatial variability in community structure of Dictyoceratida sponges across Torres Strait, Australia. Continental Shelf Research, 28, 2168-2173

Dye, A.H. (1998) Community level analysies of long-term changes in rocky littoral fauna from South Africa. Marine Ecology Progress Series, 164, 47-57

Edinger, E.N., Kolasa, J. \& Risk, M.J. (2000) Biogeographic variation in coral species diversity on coral reefs in three regions of Indonesia. Diversity and Distributions, 6, 113-127

Ellingsen, K.E., Hewitt, J.E. \& Thrush, S.F. (2007) Rare species, habitat diversity and functional redundancy in marine benthos. Journal of Sea Research, 58, 291-301

Elvin, D. (1976) Seasonal growth and reproduction of an intertidal sponge, Haliclona permollis (Bowerbank). Biological Bulletin, 151, 108-125

Fagerstrom, J.A., West, R.R., Kershaw, S. \& Cossey, P.J. (2000) Spatial competition among clonal organisms in extant and selected Paleozoic reef communities. Facies, 42, 1-24

Fahrig, L. \& Merriam, G. (1994) Conservation of fragmented populations. Conservation Biology, 8, 50-59

Faith, D.P. (2003) Environmental diversity (ED) as surrogate information for species-level biodiversity. Ecography, 26, 374-379

Fell, P. (1993) Porifera. Reproductive biology of invertebrates (ed. by K.G. Adiyodi and R.G. Adiyodi), pp. 144. Oxford \& IBH publishing Co. PVT. LTD., New Dehli.

Fell, P.E. \& Levandrowski, K.B. (1981) Population dynamics of the estuarine sponge. Halichondria sp. within a New England eelgrass community. Journal of Experimental Marine Biology and Ecology, 55, 49-63

Fell, P.E., Knight, P.A. \& Rieders, W. (1989) Low salinity tolerance of and salinity induced dormancy in the estuarine sponge Microciona prolifera (Ellis et Solander) under long-term laboratory culture. Journal of Experimental Marine Biology and Ecology, 133, 195-211

Fleishman, E., Betrus, C.J. \& Blair, R.B. (2003) Effects of spatial scale and taxonomic group on partitioning of butterfly and bird diversity in the Great Basin, USA. Landscape Ecology, 18, 675-685

Foster-Smith, J. \& Evans, S.M. (2003) The value of marine ecological data collected by volunteers. Biological Conservation, 113, 199-213

Fowler, S. \& Laffoley, D. (1993) Stability in Mediterranean-Atlantic sessile epifaunal communities at the northern limits of their range. Journal of Experimental Marine Biology and Ecology, 172, 109.

Francis, M. (1996) Geographical distribution of marine reef fishes in the New Zealand region. New Zealand Journal of Marine \& Freshwater Research, 30, 35-55 
Freeman, D., Marshall, B., Ahyong, S., Wing, S. \& Hitchmough, R. (2010) Conservation status of New Zealand marine invertebrates, 2009. New Zealand Journal of Marine and Freshwater Research, 44, 129 148

Galtsoff, P.S. (1942) Wasting disease causing mortality of sponges in the West Indies and Gulf of Mexico. Proceedings of the 8th American Science Congress, 3, 411-421

Gardner, J. \& Thompson, R.J. (2001) Naturally low seston concentration and the net energy balance of the greenshell mussel (Perna canaliculus) at Island Bay, Cook Strait, New Zealand. New Zealand Journal of Marine \& Freshwater Research, 35, 457-468

Gardner, J.P.A. (2000) Where are the mussels on Cook Strait (New Zealand) shores? Low seston quality as a possible factor limiting multi-species distributions. Marine Ecology Progress Series, 194, 123132

Garrabou, J. \& Zabala, M. (2001) Growth dynamics in four Mediterranean demosponges. Estuarine, Coastal and Shelf Science, 52, 293-303

Garson, J., Aggarwal, A. \& Sarkar, S. (2002) Birds as surrogates for biodiversity: An analysis of a data set from southern Québec. Journal of Biosciences, 27, 347-360

Gaston, K. (2000) Global patterns in biodiversity. Nature, 405, 6783

Gaston, K.J. (2010) Valuing common species. Science, 327, 154-155

Gaston, K.J. \& Fuller, R.A. (2008) Commonness, population depletion and conservation biology. Trends in Ecology \& Evolution, 23, 14-19

Gaston, K.J., Rodrigues, A.S.L., Van Rensburg, B.J., Koleff, P. \& Chown, S.L. (2001) Complementary representation and zones of ecological transition. Ecology Letters, 4, 4-9

Gaymer, C.F., Palma, A.T., Vega, J.M.A., Monaco, C.J. \& Henríquez, L.A. (2010) Effects of La Niña on recruitment and abundance of juveniles and adults of benthic community-structuring species in northern Chile. Marine \& Freshwater Research, 61, 1185-1196

Gering, J.C., Crist, T.O. \& Veech, J.A. (2003) Additive partitioning of species diversity across multiple spatial scales: Implications for regional conservation of biodiversity. Conservation Biology, 17, 488-499

Ghiold, J., Rountree, G.A. \& Smith, S.H. (1994) Common sponges of the Cayman Islands. The Cayman Islands: Natural History and Biogeography, 71, 131-138

Gladstone, W. \& Alexander, T. (2005) A test of the higher-taxon approach in the identification of candidate sites for marine reserves. Biodiversity and Conservation, 14, 3151-3168

Goldberg, N.A., Kendrick, G.A. \& Walker, D.I. (2006) Do surrogates describe patterns in marine macroalgal diversity in the Recherche Archipelago, temperate Australia? Aquatic Conservation-Marine and Freshwater Ecosystems, 16, 313-327

Gonzalez, A. \& Loreau, M. (2009) The causes and consequences of compensatory dynamics in ecological communities. Annual Review of Ecology, Evolution, and Systematics, 40, 393-414.

Gordon, D.P. (2009) New bryozoan taxa from a new marine conservation area in New Zealand, with a checklist of Bryozoa from Greater Cook Strait. Zootaxa, 1987, 39-60

Gordon, D.P., Beaumont, J., MacDiarmid, A., Robertson, D.A. \& Ahyong, S.T. (2010) Marine Biodiversity of Aotearoa New Zealand. PLoS ONE, 5, e10905

Gotelli, N.J. \& Colwell, R.K. (2001) Quantifying biodiversity: procedures and pitfalls in the measurement and comparison of species richness. Ecology Letters, 4, 379-391

Grantham, H.S., Pressey, R.L., Wells, J.A. \& Beattie, A.J. (2010) Effectiveness of biodiversity surrogates for conservation planning: Different measures of effectiveness generate a kaleidoscope of variation. PLOS ONE, 5, e11430 
References

Griffiths, G.A. (1979) High sediment yields from major rivers of the western Southern Alps, New Zealand Nature, 282, 61-63

Hanson, C.E., McLaughlin, M.J., Hyndes, G.A. \& Strzelecki, J. (2009) Selective uptake of prokaryotic picoplankton by a marine sponge (Callyspongia sp.) within an oligotrophic coastal system. Estuarine, Coastal and Shelf Science, 84, 289-297

Harper, M.A., Patterson, J.E. \& Harper, J.F. (2009) New diatom taxa from the world's first marine Bioblitz held in New Zealand: Skeletomastus a new genus, Skeletomastus coelatus nov. comb. and Pleurosigma inscriptura a new species. Acta Botanica Croatica, 68, 339-349

Harsha, R.E., Francis, J.C. \& Poirrier, M.A. (1983) Water temperature: a factor in the seasonality of two freshwater sponge species, Ephydatia fluviatilis and Spongilla alba. Hydrobiologia, 102, 145-150

Hartman, W.D. (1958) Natural history of the marine sponges of southern New England. Bulletin of the Peabody Museum of Natural History, Yale University, 12, 1-155

Hastie, T. \& Tibshirani, R. (1990) Generalized additive models. Chapman and Hall.

Hay, N. (2008) Monitoring changes in Indonesian coral reefs using macrobenthic assemblage variability. Victoria University of Wellington, Wellington.

Heath, R.A. (1971) Hydrology and circulaiton in central and southern Cook Strait, New Zealand. New Zealand Journal of Marine \& Freshwater Research, 5, 178-99

Heath, R.A. (1985) A review of the physical oceanography of the seas around New Zealand. New Zealand Journal of Marine and Freshwater Research, 19, 79-124

Helson, J.G., Pledger, S. \& Gardner, J.P.A. (2007) Does differential particulate food supply explain the presence of mussels in Wellington Harbour (New Zealand) and their absence on neighbouring Cook Strait shores? Estuarine, Coastal and Shelf Science, 72, 223-234

Henry, L.A. \& Hart, M. (2005) Regeneration from injury and resource allocation in sponges and corals - A review. International Review of Hydrobiology, 90, 125-158

Hinde, G.J. \& Holmes, W.M. (1892) On the sponge-remains in the lower tertiary strata near Oamaru, Otago, New Zealand. Linnean Society Journal of Zoology, 24, 177-263

Hiscock, K. (1994) Marine communities at Lundy - origins, longevity and change. Biological Journal of the Linnean Society, 51, 183-188

Hooper, D.U., Chapin, F.S.I.I.I., Ewel, J.J., Hector, A., Inchausti, P., Lavorel, S., J. H. Lawton, Lodge, D.M., Loreau, M., Naeem, S., Schmid, B., Setälä, H., Symstad, A.J., Vandermeer, J. \& Wardle, D.A. (2005) Effects of biodiversity on ecosystem functioning: a consensus of current knowledge Ecological Monographs, 75, 3-35

Hooper, J.N.A. \& Van Soest, R.W.M. (2002) Systema Porifera: A guide to the classification of sponges. Kulwer Academic/ Plenum Publishers, London.

Hooper, J.N.A., Kennedy, J.A. \& Quinn, R.J. (2002) Biodiversity 'hotspots', patterns of richness, endemism, and taxonomic affinities of tropical Australian sponges (Porifera). Biodiversity and Conservation, 11, $851-885$

Hoppe, W.F. (1988) Growth, regeneration and predation in three species of large coral reef sponges. Marine Ecology Progress Series, 50, 117-125

Hoshino, T. (1981a) Shallow-water demosponges of western Japan, I. Journal of Science of the Hiroshima University, 29, 47-205

Hoshino, T. (1981b) Shallow-water demosponges of western Japan II. Journal of Science of the Hiroshima University, 29, 207-289 
References

Hubbard, R. (1990) Sponges (Porifera) of the order Dictyoceratida, Dendroceratida, and Verongiida (class Demospongiae) from Trinidad and Tobago. Caribbean Marines studies, 1, 54-67

Hughes, T.P. (1996) Demographic approaches to community dynamics: a coral reef example. Ecology, 77, 2256-2260

Hutchings, P.A., Hilliard, R.W. \& Coles, S.L. (2002) Species introductions and potential for marine pest invasions into tropical marine communities, with special reference to the Indo-Pacific. Pacific Science, 56, 223-233

Ilan, M. \& Abelson, A. (1995) The life of a sponge in a sandy lagoon. Biological Bulletin, 189, 363-369

Inglis, G., Gust, N., Fitridge, I., Floerl, O., Woods, C., Hayden, B. \& Fenwick, G. (2005a) Dunedin Harbour (Port Otago and Port Chalmers). Baseline survey for non-indigenous marine species. In. Biosecurity New Zealand

Inglis, G., Gust, N., Fitridge, I., Floerl, O., Woods, C., Hayden, B. \& Fenwick, G. (2005b) Port of Bluff. Baseline survey for non-indigenous marine species. In: (ed. M.O.A.A. Forestry). Biosecurity New Zealand NIWA

Inglis, G., Gust, N., Fitridge, I., Floerl, O., Woods, C., Hayden, B. \& Fenwick, G. (2005c) Port of Picton. Baseline survey for non-indigenous marine species. In: (ed. B.N. Zealand). Ministry for Agriculture and Forestry

Inglis, G., Gust, N., Fitridge, I., Floerl, O., Woods, C., Hayden, B. \& Fenwick, G. (2005d) Port of Tauranga. Baseline survey for non-indigenous marine species. In. Biosecurity New Zealand

Inglis, G., Gust, N., Fitridge, I., Floerl, O., Woods, C., Hayden, B. \& Fenwick, G. (2005e) Port of Wellington. Baseline survey for non-indigenous marine species. In. Biosecurity New Zealand

Inglis, G., Gust, N., Fitridge, I., Floerl, O., Woods, C., Hayden, B. \& Fenwick, G. (2005f) Whangarei Harbour (Whangarei Port and Marsden Point). Baseline survey for non-indigenous marine species. In. Biosecurity New Zealand

Inglis, G., Gust, N., Fitridge, I., Floerl, O., Woods, C., Hayden, B. \& Fenwick, G. (2008a) Port of Lyttelton. Second baseline survey for non-indigenous marine species (Research Project ZBS2000-04). In. NIWA

Inglis, G., Gust, N., Fitridge, I., Floerl, O., Woods, C., Kospartov, M., Hayden, B. \& Fenwick, G. (2008b) Port of Tauranga. Second baseline survey for non-indigenous marine species (Research Project ZBS2000/04). MAF Biosecurity New Zealand Technical Paper, 1-141

Inglis, G., Gust, N., Fitridge, I., Floerl, O., Woods, C., Kospartov, M., Hayden, B. \& Fenwick, G. (2008c) Port of Wellington. Second baseline survey for non-indigenous marine species (Research Project ZBS2000/04). MAF Biosecurity New Zealand Technical Paper, 1-145

Inglis, G., Gust, N., Fitridge, I., Morrisey, D., Floerl, O., Woods, C., Kospartov, M., Hayden, B. \& Fenwick, G. (2008d) Port of Nelson Second baseline survey for non-indigenous marine species (Research Project ZBS2000-04). In: MAF Biosecurity New Zealand Technical Paper

Inglis, G., Gust, N., Fitridge, I., Morrisey, D., Floerl, O., Woods, C., Kospartov, M., Hayden, B. \& Fenwick, G. (2008e) Port of Picton. Second baseline survey for non-indigenous marine species (Research Project ZBS2008/04). MAF Biosecurity New Zealand Technical Paper, 2008/04

Jackson, J.B.C. (1977) Competition on marine hard substrata: The adaptative significance of solitary and colonial strategies. The American Naturalist, 111, 743-767.

Jackson, J.B.C. (2008) Ecological extinction and evolution in the brave new ocean. Proceedings of the National Academy of Sciences, 105, 11458-11465 
Janussen, D. \& Tendal, O.S. (2007) Diversity and distribution of Porifera in the bathyal and abyssal Weddell Sea and adjacent areas. Deep Sea Research Part II: Topical Studies in Oceanography, 54, 18641875.

Jetz, W., Kreft, H., Ceballos, G. \& Mutke, J. (2009) Global associations between terrestrial producer and vertebrate consumer diversity. Proceedings of the Royal Society B: Biological Sciences, 276, 269278

Johnson, M.F. (1979) Recruitment, growth. mortality and seasonal variations in the calcareous sponges Clathrina coriacea (Montagu) and C. blanca (Miklucho-Maclay) from Santa Catalina Island, California. Biologie des Spongiaires 7th Colloques internationaux du Centre National dela Recherche Scientifique (ed. by C. Lévi and N. Boury-Esnault), pp. 325-334. Editions du C.N.R.S., Paris.

Jokiel, P.L. (1980) Solar ultraviolet radiation and coral reef epifauna. Science (U.S.), 207, 1069-1071

Jones, A.J. \& Garrick, A.S. (1991) Tuhua (Mayor Island) marine habitats. A preliminary intertidal and subtidal survey. In: Technical Report Series. Department of Conservation, Bay of Plenty Conservancy, Rotorua

Jones, T.C., Gemmill, C.E.C. \& Pilditch, C.A. (2008) Genetic variability of New Zealand seagrass (Zostera muelleri) assessed at multiple spatial scales. Aquatic Botany, 88, 39-46

Jost, L. (2007) Partitioning diversity into independent alpha and beta components. Ecology, 88, 2427-2439

Jost, L., DeVries, P., Walla, T., Greeney, H., Chao, A. \& Ricotta, C. (2009) Partitioning diversity for conservation analyses. Diversity and Distributions, 16, 65-76

Kaandorp, J.A. (1991) Modelling growth forms of the sponge Haliclona oculata (Porifera, Demospongiae) using fractal techniques. Marine Biology, 110, 203-215

Kaandorp, J.A. (1995) Analysis and synthesis of radiate accretive growth in three dimensions. Journal of Theoretical Biology, 175, 39-55

Karlson, R.H., Cornell, H.V. \& Hughes, T.P. (2004) Coral communities are regionally enriched along an oceanic biodiversity gradient. Nature, 429, 867-870

Kattan, G.H., Franco, P., Saavedra-RodrÍguez, C.A., Valderrama, C., Rojas, V., Osorio, D. \& MartÍnez, J. (2006) Spatial components of bird diversity in the Andes of Colombia: implications for designing a regional reserve system. Componentes espaciales de la Diversidad de Aves en los Andes de Colombia: Implicaciones para el Diseño de un Sistema Regional de Reservas. Conservation Biology, 20, 1203-1211

Kefalas, E., Tsirtsis, G. \& Castritsi-Catharios, J. (2003) Distribution and ecology of Demospongiae from the circalittoral of the islands of the Aegean Sea (Eastern Mediterranean). Hydrobiologia, 499, 125134

Kelly, M. (2000) Description of a new lithistid sponge from northeastern New Zealand, and consideration of the phylogenetic affinities of families Corallistidae and Neopeltidae. Zoosystema, 22, 265-283

Kelly, M. (2003) *Revision of the sponge genus Pleroma Sollas (Lithistida : Megamorina : Pleromidae) from New Zealand and New Caledonia, and description of a new species. New Zealand Journal of Marine and Freshwater Research, 37, 113-127

Kelly, M. (2007) The marine fauna of New Zealand: Porifera: Lithistid Demospongiae (rock sponges). NIWA, Wellington.

Kelly, M., Edwards, A.R., Wilkinson, M.R., Alvarez, B., Cook, S.C., Bergquist, P.R., Buckeridge, J.S., Campbell, H., Reiswig, H.M. \& Valentine, C. (2009) Phylum Porifera Sponges. The New Zealand Inventory of 
Biodiversity Volume 1. Kingdom Animalia: Radiata, Lophotrochozoa, and Deuterostomia. (ed. by D.P. Gordon), p. 584. Canterbury University Press, Christchurch.

Kennelly, S.J. \& Underwood, A.J. (1992) Fluctuations in the distribution and abundance of species in sublittoral kelp forests in New South Wales. Australian Journal of Ecology, 17, 367-82.

Kerswell, A.P. (2006) Global biodiversity patterns of benthic marine algae. Ecology, 87, 2479-2488.

Kingsford, M.J. \& Milicich, M.J. (1987) Presettlement phase of Parika scaber (Pisces:Monacanthidae): a temperate reef fish. Marine Ecology Progress Series, 36, 65-79

Klautau, M. \& Valentine, C. (2003) Revision of the genus Clathrina (Porifera, Calcarea). Zoological Journal of the Linnean Society, 139, 1-62

Klimek, S., Marini, L., Hofmann, M. \& Isselstein, J. (2008) Additive partitioning of plant diversity with respect to grassland management regime, fertilisation and abiotic factors. Basic and Applied Ecology, 9, 626-634

Knowlton, A.L. \& Highsmith, R.C. (2005) Nudibranch-sponge feeding dynamics: Benefits of symbiontcontaining sponge to Archidoris montereyensis (Cooper, 1862) and recovery of nudibranch feeding scars by Halichondria panicea (Pallas, 1766). Journal of Experimental Marine Biology \& Ecology, 327, 36-46

Knox, G.A. (1975) The marine benthic ecology and biogeography. Biogeography and ecology in New Zealand (ed. by G. Kuschel), pp. 353-403. Junk W., The Hague.

Koopmans, M. \& Wijffels, R.H. (2008) Seasonal growth rate of the sponge Haliclona oculata (Demospongiae: Haplosclerida). Marine Biotechnology, 10, 502-510

Kunin, W.E. \& Gaston, K.J. (1993) The biology of rarity: Patterns, causes and consequences. Trends in Ecology \& Evolution, 8, 298-301

Labate, M. (1964) Poriferi di grotta superficiale del litorale adriatico pugliese. Annali del Pontificio Istituto superiore di Scienze e Lettere "S Chiara", 14, 319-342

Lande, R. (1996) Statistics and Partitioning of Species Diversity, and Similarity among Multiple Communities. Oikos, 76, 5-13

Landis, C.A., Campbell, H.J., Begg, J.G., Mildenhall, D.C., Paterson, A.M. \& Trewick, S.A. (2008) The Waipounamu Erosion Surface: questioning the antiquity of the New Zealand land surface and terrestrial fauna and flora. Geological Magazine, 145, 173-197

Lanna, E., Cavalcanti, F.F., Cardoso, L., Muricy, G. \& Klautau, M. (2009) Taxonomy of calcareous sponges (Porifera, Calcarea) from Potiguar Basin, NE Brazil. Zootaxa, 1-27

Lawton, J.H. (1999) Are There General Laws in Ecology? Oikos, 84, 177-192

Lawton, J.H. \& Brown, V.K. (1993) Redundancy in ecosystems. Biodiversity and ecosystem function (ed. by E.D. Schulze and H.A. Mooney), pp. 255-270. Springer Verlag, New York.

Leamon, J. \& Fell, P.E. (1990) Upper salinity tolerance of and salinity-induced tissue regression in the estuarine sponge Microciona prolifera. Transactions of the American microscopical Society, 109, 265-272

Lehnert, H. \& Soest, R.W.M.V. (1996) North Jamaican deep fore-reef sponges. Beaufortia, 46, 53-81

Lehnert, H. \& Soest, R.W.M.V. (1998) Shallow-water sponges of Jamaica. Beaufortia, 48, 71-103

Leichter, J.J. \& Witman, J.D. (1997) Water flow over subtidal rock walls: relation to distributions and growth rates of sessile suspension feeders in the Gulf of Maine Water flow and growth rates. Journal of Experimental Marine Biology and Ecology, 209, 293-307

Lemloh, M.L., Fromont, J., Brümmer, F. \& Usher, K.M. (2009) Diversity and abundance of photosynthetic sponges in temperate Western Australia. BMC Ecology, 9, 4-9 
Leong, W. \& Pawlik, J.R. (2010) Fragments or propagules? Reproductive tradeoffs among Callyspongia spp. from Florida coral reefs. Oikos, 119, 1417-1422

Lévi, C., Laboute, P., Bargibant, G., Menou, J.L., Battershill, C., Bergquist, P., Borojevic, R., Debitus, C., Fromont, J., Hooper, J., Kelly-Borges, M., Vacelet, J. \& Wilkinson, C. (1998) Sponges of the New Caledonian lagoon ORSTOM, Paris.

Leys, S.P., Wilson, K., Holeton, C., Reiswig, H.M. \& Austin, W.C. (2004) Patterns of glass sponge (Porifera, Hexactinellida) distribution in coastal waters of British Columbia. Marine Ecology Progress Series, 283, 133-149

Likens, G.E. (1989) Long term studies in ecology. Springer-Verlag, New York.

Lind, C.E., Evans, B.S., Taylor, J.J.U. \& Jerry, D.R. (2007) Population genetics of a marine bivalve, Pinctada maxima, throughout the Indo-Australian Archipelago shows differentiation and decreased diversity at range limits. Molecular Ecology, 16, 5193-5203

Lock, K., Burton, M., Gibbs, R. \& Newman, P. (2009) Skomer marine nature reserve project status report 2008/9. In: CCW Regional Report, p. 83

Lock, K., Burton, M., Gibbs, R. \& Newman, P. (2010a) Skomer marine nature reserve project status report 2009/10. In: CCW Regional Report, p. 86

Lock, K., Newman, P. \& Burton, M. (2010b) Skomer Marine Nature Reserve nudibranch diversity survey 2010 CCW In: CCW Regional Report, p. 36.

Logan, J.A., Megretskaia, I., Nassar, R., Murray, L.T., Zhang, L., Bowman, K.W., Worden, H.M. \& Luo, M. (2008) Effects of the 2006 El Niño on tropospheric composition as revealed by data from the Tropospheric Emission Spectrometer (TES). Geophysical Research Letters, 35, L03816.

Lubchenco, J., Palumbi, S.R., Gaines, S.D. \& Andelman, S. (2003) Plugging a hole in the ocean: the emerging science of marine reserves. Ecological Applications, 13, S3-S7

Luna, B., Pãrez, C.V. \& Sãnchez-Lizaso, J.L. (2009) Benthic impacts of recreational divers in a Mediterranean Marine Protected Area. ICES Journal of Marine Science: Journal du Conseil, 66, 517-523

MacArthur, R., Recher, H. \& Cody, M. (1966) On the relation between habitat selection and species diversity. The American Naturalist, 100, 319-332

Mace, G.M. (2004) The role of taxonomy in species conservation. Philosophical Transactions: Biological Sciences, 359, 711-719

Magurran, A.E. \& Henderson, P.A. (2003) Explaining the excess of rare species in natural species abundance distributions. Nature, 422, 714-716

Magurran, A.E. \& Henderson, P.A. (2010) Temporal turnover and the maintenance of diversity in ecological assemblages. Philosophical Transactions of the Royal Society B: Biological Sciences, 365, 36113620

Maldonado, M. (2006) The ecology of the sponge larva. Canadian Journal of Zoology, 84, 175-194

Maldonado, M. \& Young, C.M. (1996) Effects of physical factors on larval behavior, settlement and recruitment of four tropical demosponges. Marine Ecology Progress Series, 138, 169-180

Manconi, R. \& Pronzato, R. (2008) Global diversity of sponges (Porifera: Spongillina) in freshwater. Hydrobiologia, 595, 27-33

Margules, C.R. \& Pressey, R.L. (2000) Systematic conservation planning. Nature, 405, 243-253

Mariani, S., Uriz, M.-J., Turon, X. \& Alcoverro, T. (2006) Dispersal strategies in sponge larvae: integrating the life history of larvae and the hydrologic component. Oecologia, 149, 174-184.

Mariani, S., Uriz, M.J. \& Turon, X. (2005) The dynamics of sponge larvae assemblages from northwestern Mediterranean nearshore bottoms. Journal of Plankton Research, 27, 249-262 
May, R.M. (1990) Taxonomy as destiny. Nature., 347, 129-130

McArthur, M.A., Brooke, B.P., Przeslawski, R., Ryan, D.A., Lucieer, V.L., Nichol, S., Mccallum, A.W., Mellin, C., Cresswell, I.D. \& Radke, L.C. (2010) On the use of abiotic surrogates to describe marine benthic biodiversity. Estuarine, Coastal and Shelf Science, 88, 21-32

McCann, K.S. (2000) The diversity-stability debate. Nature, 405, 228-233

McClintock, J.B., Amsler, C.D., Baker, B.J. \& van Soest, R.W.M. (2005) Ecology of antarctic marine sponges: An overview. Integrative and Comparative Biology, 45, 359-368

McMurray, S.E., Blum, J.E. \& Pawlik, J.R. (2008) Redwood of the reef: growth and age of the giant barrel sponge Xestospongia muta in the Florida Keys. Marine Biology, 1-13

Meroz-Fine, E., Shefer, S. \& Ilan, M. (2005) Changes in morphology and physiology of an East Mediterranean sponge in different habitats. Marine Biology, 147, 243-250

Miller, K., Alvarez, B., Battershill, C., Northcote, P. \& Parthasarathy, H. (2001) Genetic, morphological, and chemical divergence in the sponge genus Latrunculia (Porifera: Demospongiae) from New Zealand. Marine Biology, 139, 235-250

Miller, K.J. (1998) Short-distance dispersal of black coral larvae: inference from spatial analysis of colony genotypes. Marine Ecology Progress Series, 163, 225-233

MoAF/DoC (1971) Marine Reserves Act. In: (ed. M.O.A.A.F.A.T.D.O. Conservation)

Moore, L.B. (1949) The marine algal provinces of New Zealand. Transactions of the Royal Society of New Zealand, 77, 187-689

Mortimer, N., Hoernle, K., Hauff, F., Palin, J.M., Dunlap, W.J., Werner, R. \& Faure, K. (2006) New constraints on the age and evolution of the Wishbone Ridge, southwest Pacific Cretaceous microplates, and Zealandiaâ€"West Antarctica breakup. Geology, 34, 185-188

Navarrete, S.A., Broitman, B., Wieters, E.A., Finke, G.R., Venegas, R.M. \& Sotomayor, A. (2002) Recruitment of intertidal invertebrates in the Southeast Pacific: interannual variability and the 1997-1998 El Niño. Limnology and Oceanography, 47, 791-802

Nelson, W. (1994) Distribution of macroalgae in New Zealand - an archipelago in space and time. Botanica Marina, 37, 221-233

Nelson, W. (2008) Macroalgae of the Wellington South Coast. The Taputeranga marine reserve (ed. by J.P.A. Gardner and J.J. Bell), pp. 196-214, Wellington.

Nielsen, S., Haughland, D., Bayne, E. \& Schieck, J. (2009) Capacity of large-scale, long-term biodiversity monitoring programmes to detect trends in species prevalence. Biodiversity and Conservation, 18, 2961-2978

NIWA (2011) NIWA's National Climate Database on the Web (accessed on 25/03/2011). Available at: http://cliflo.niwa.co.nz/ (accessed 25-March 2011).

Økland, K.A. \& Økland, J. (1996) Freshwater sponges (Porifera: Spongillidae) of Norway: distribution and ecology. Hydrobiologia, 330, 1-30

Orton, J.H. (1914) Preliminary account of a contribution to an evaluation of the sea. Journal of the Marine Biological Association of the United Kingdom , 10, 313-326

Page, M., West, L., Northcote, P., Battershill, C. \& Kelly, M. (2005) Spatial and temporal variability of cytotoxic metabolites in populations of the New Zealand sponge Mycale hentscheli. Journal of Chemical Ecology, 31, 1161-1174

Page, M.J., Handley, S.J., Northcote, P.T., Cairney, D. \& Willan, R.C. (2010) Successes and pitfalls of the aquaculture of the sponge Mycale hentscheli. Aquaculture, 312, 52-61 
References

Pande, A. (2001) Evaluating biological change in New Zealand marine reserves. PhD thesis. Victoria University of Wellington, Wellington.

Pande, A. \& Gardner, J.P.A. (2009) A baseline biological survey of the proposed Taputeranga Marine Reserve (Wellington, New Zealand): spatial and temporal variability along a natural environmental gradient. Aquatic Conservation: Marine and Freshwater Ecosystems, 19, 237-248

Pande, A., MacDiarmid, A., J., S.P., Davidson, R.J., Cole, R.G., Freeman, D., Kelly, S. \& Gardner, J.P.A. (2008) Marine reserves increase the abundance and size of blue cod and rock lobster. Marine Ecology Progress Series, 366, 147-158

Pandolfi, J.M., Bradbury, R.H., Sala, E., Hughes, T.P., Bjorndal, K.A., Cooke, R.G., Mcardle, D., Mcclenachan, L., Newman, M.J.H., Paredes, G., Warner, R.R. \& Jackson, J.B.C. (2003) Global trajectories of the longterm decline of coral reef ecosystems. Science, 301, 955-958

Pang, R.K. (1973) The ecology of some Jamaican excavating sponges. Bulletin of Marine Science, 23, 227243.

Pansini, M. \& Longo, C. (2003) A review of the Mediterranean sea sponge biogeography with a list of the demosponges hitherto recorded from this sea. Biogeographia, 24, 59-90

Pansini, M. \& Pronzato, R. (1985) Observations on the dynamics of a Mediterranean sponge community. New Perspectives in Sponge Biology (ed. by K. Rützler), pp. 404-415. Smithsonian Institution Press, London.

Perea-Blázquez, A. (2011) Interactions between sponges and the water column: utilisation of food resources and nutrient uptake by New Zealand subtidal sponges. $\mathrm{PhD}$ thesis. Victoria University of Wellington, Wellington.

Perea-Blázquez, A., Price, K., Davy, S.K. \& Bell, J.J. (2010) Diet Composition of Two Temperate Calcareous Sponges: Leucosolenia echinata and Leucetta sp. from the Wellington South Coast, New Zealand. The Open Marine Biology Journal, 4, 65-73

Perez, T., Longet, D., Schembri, T., Rebouillon, P. \& Vacelet, J. (2005) Effects of 12 years' operation of a sewage treatment plant on trace metal occurrence within a Mediterranean commercial sponge (Spongia officinalis, Demospongiae). Marine Pollution Bulletin, 50, 301-309

Petchey, O.L. \& Gaston, K.J. (2002) Functional diversity (FD), species richness and community composition. Ecology Letters, 5, 402-411

Pickrill, R.A. (1993) Sediment yields in Fiordland. Journal of Hydrology (NZ), 31, 39-55

Picton, B.E. \& Goodwin, C.E. (2007) Sponge biodiversity of Rathlin Island, Northern Ireland. Journal of the Marine Biological Association of the United Kingdom, 87, 1441-1458

Pienkowski, M.W., Watkinson, A.R., Kerby, G., Warwick, R.M. \& Clarke, K.R. (1998) Taxonomic distinctness and environmental assessment. Journal of Applied Ecology, 35, 532-543

Pile, A.J. (1996) Finding Reiswig's missing carbon: quantification of sponge feeding using dual-beam flow cytometry. 8th International Coral Reef Symposium, June 24-29, 1996, Panama, abstract (ed. by H.A. Lessios), p. 158. ICRS, Panama.

Pile, A.J., Patterson, M.R. \& Witman, J.D. (1996) In situ grazing on plankton $<10 \mu \mathrm{m}$ by the boreal sponge Mycale lingua. Marine Ecology Progress Series, 141, 95-102

Plotkin, A. (2004) Biodiversity and distribution of Polymastiidae (Demospongiae, Hadromerida) in the Arctic Area. Bollettino dei Musei e degli Istituti Biologici della Universita di Genova, 68, 535-547

Pöppe, J., Sutcliffe, P., Hooper, J.N.A., Wörheide, G. \& Erpenbeck, D. (2010) CO I Barcoding Reveals New Clades and Radiation Patterns of Indo-Pacific Sponges of the Family Irciniidae (Demospongiae: Dictyoceratida). PLOS ONE, 5, e9950 
References

Poulin, E., Palma, A.T., Leiva, G., Narvaez, D., Pacheco, R., Navarrete, S.A. \& Castilla, J.C. (2002) Avoiding offshore transport of competent larvae during upwelling events: The case of the gastropod Concholepas concholepas in Central Chile. Limnology and Oceanography [Limnol. Oceanogr.]. 47, 1248-1255

Powell, A.L., Hepburn, L.J., Smith, D.J. \& Bell, J.J. (2010) Patterns of sponge abundance across a gradient of habitat quality in the Wakatobi Marine National Park, Indonesia. The Open Marine Biology Journal, 4, 31-38

Pressey, R.L. (2004) Conservation planning and biodiversity: assembling the best data for the job. Conservation Biology, 18, 1677-1681

Pritchard, K., Ward, V., Battershill, C.N. \& Bergquist, P.R. (1984) Marine sponges: forty-six sponges of northern New Zealand. Leigh Laboratory Bulletin, 14, 1-149

Pronzato, R. (1999) Sponge-fishing, disease and farming in the Mediterranean Sea. Aquatic conservation, marine and freshwater ecosystems, 9, 485-493.

Pronzato, R. (1999) Sponge-fishing, disease and farming in the Mediterranean Sea. Aquatic conservation, marine and freshwater ecosystems, 9, 485-493

Pulitzer-Finali, G. \& Pronzato, R. (1999) Horny sponges from the north-eastern coast of Papua New Guinea, Bismark Sea. Journal of the Marine Biological Association of the United Kingdom, 79, 593-607

Rapp, H.T. (2006) Calcareous sponges of the genera Clathrina and Gauncha (Calcinea, Calcarea, Porifera) of Norway (north east Atlantic) with the description of five new species. Zoological Journal of the Linnean Society, 147, 331-365

Reaka, M.L., Rodgers, P.J. \& Kudla, A.U. (2008) Patterns of biodiversity and endemism on Indo-West Pacific coral reefs. Proceedings of the National Academy of Sciences, 105, 11474-11481

Reiswig, H.M. (1973) Population dynamics of three Jamaican demospongiae. Bulletin of Marine Science, 23, 191-226

Reiswig, H.M. (1974) Water transport, respiration and energetics of three tropical marine sponges. Journal of Experimental Marine Biology and Ecology, 14, 231-249

Ribeiro, S.M., Omena, E.P. \& Muricy, G. (2003) Macrofauna associated to Mycale microsigmatosa (Porifera, Demospongiae) in Rio de Janeiro State, SE Brazil. Estuarine, Coastal and Shelf Science, 57, 951959

Ribes, M., Coma, R. \& Gili, J.M. (1999) Natural diet and grazing rate of the temperate sponge Dysidea avara (Demospongiae, Dendroceratida) throughout an annual cycle. Marine Ecology Progress Series, 176, 179-190

Ribes, M., Coma, R., Atkinson, M.J. \& Kinzie Iii, R.A. (2003) Particle removal by coral reef communities: Picoplankton is a major source of nitrogen. Marine Ecology Progress Series, 257, 13-23

Riisgard, H.U., Thomassen, S., Jakobsen, H., Weeks, J.M. \& Larsen, P.S. (1993) Suspension feeding in marine sponges Halichondria panicea and Haliclona urceolus: effects of temperature on filtration rate and energy cost of pumping. Marine Ecology Progress Series, 96, 177-188

Roberts, C.M., McClean, C.J., Veron, J.E.N., Hawkins, J.P., Allen, G.R., McAllister, D.E., Mittermeier, C.G., Schueler, F.W., Spalding, M., Wells, F., Vynne, C. \& Werner, T.B. (2002) Marine Biodiversity Hotspots and Conservation Priorities for Tropical Reefs. Science, 295, 1280-1284

Roberts, D.E. \& Davis, A.R. (1996) Patterns in Sponge (Porifera) assemblages on temperate coastal reefs off Sydney, Australia. Marine and Freshwater Research, 47, 897-906 
References

Roberts, D.E., Cummins, S.P., Davis, A.R. \& Chapman, M.P. (2006) Structure and dynamics of spongedominated assemblages on exposed and sheltered temperate reefs. Marine Ecology Progress Series, 321, 19-30

Rodgers, K.L. \& Wing, S.R. (2008) Spatial structure and movement of blue cod Parapercis colias in Doubtful Sound, New Zealand, inferred from delta super(13)C and delta super(15)N Marine Ecology Progress Series, 359, 239-248

Rondinini, C., Wilson, K.A., Boitani, L., Grantham, H. \& Possingham, H.P. (2006) Tradeoffs of different types of species occurrence data for use in systematic conservation planning. Ecology Letters, 9, 11361145

Rosell, D. \& Uriz, M.J. (1992) Do associated zooxanthellae and the nature of the substratum affect survival, attachment and growth of Cliona viridis (Porifera: Hadromerida)? An experimental approach. Marine Biology, 114, 503-507

Ross, P.M., Hogg, I.D., Pilditch, C.A. \& Lundquist, C.J. (2009) Phylogeography of New Zealand's coastal benthos. New Zealand Journal of Marine and Freshwater Research, 43, 1009 - 1027

Roughgarden, J., Gaines, S. \& Possingham, H. (1988) Recruitment dynamics in complex life cycles. Science, 241, 1460-1466

Rowden, A.A., Warwick, R.M. \& Gordon, D.P. (2004) Bryozoan biodiversity in the New Zealand region and implications for marine conservation. Biodiversity and Conservation, 13, 2695-2721

Russell, B.C. (1983) The food and feeding habits of rocky reef fish of north eastern New Zealand. New Zealand Journal of Marine \& Freshwater Research, 17, 121-145

Rutherford, S., D'Hondt, S. \& Prell, W. (1999) Environmental controls on the geographic distribution of zooplankton diversity. Nature, 400, 749-753

Rützler, K. (1975) The role of burrowing sponges in bioerosion. Oecologia, 19, 203-216

Rützler, K. (1978) Sponges in coral reefs. Coral reefs: research methods, (ed. by J.A. Stoddart and R.E. Johannes). UNESCO, Paris.

Rützler, K., Duran, S. \& Piantoni, C. (2007) Adaptation of reef and mangrove sponges to stress: Evidence for ecological speciation exemplified by Chondrilla caribensis new species (Demospongiae, Chondrosida). Marine Ecology, 28, 95-111

Ruzicka, R. \& Gleason, D. (2008) Latitudinal variation in spongivorous fishes and the effectiveness of sponge chemical defenses. Oecologia, 154, 785-794

Ruzicka, R. \& Gleason, D.F. (2009) Sponge community structure and anti-predator defenses on temperate reefs of the South Atlantic Bight. Journal of Experimental Marine Biology and Ecology, 380, 36-46

Sala, O.E., Stuart Chapin , F., Iii, Armesto, J.J., Berlow, E., Bloomfield, J., Dirzo, R., Huber-Sanwald, E., Huenneke, L.F., Jackson, R.B., Kinzig, A., Leemans, R., Lodge, D.M., Mooney, H.A., Oesterheld, M.Ì.N., Poff, N.L., Sykes, M.T., Walker, B.H., Walker, M. \& Wall, D.H. (2000) Global Biodiversity Scenarios for the Year 2100. Science, 287, 1770-1774

Sara, M. (1970) Competition and cooperation in sponge populations. The biology of the Porifera 7th Symposia of the Zoological Society of London (ed. by W.G. Fry), pp. 273-284. Academic Press, London.

Sara, M. (2004) Sponge peculiarities and their impact on general biology at the threshold of 2000. Bollettino dei Musei e degli Istituti Biologici della Universita di Genova, 68, 149-159

Sara, M. \& Vacelet, J. (1973) Ecologie des Démosponges. Spongiaires Traité de Zoologie (ed. by P.P. Grassé), pp. 462-576. Masson, Paris. 
Scaps, P., Denis, V., Berhimpon, S. \& Runtukahu, F. (2007) High mushroom coral biodiversity (Scleractinia: Fungiidae) in the region of Pulisan (north-eastern coast of Sulawesi, Indonesia). Forte biodiversite en coraux-champignons (scleractiniaires: Fungiides) dans la region de Pulisan (nord-est de l'ile de Sulawesi, Indonesie), 132, 87-110

Schindel, D.E. \& Miller, S.E. (2005) DNA barcoding a useful tool for taxonomists. Nature, $\mathbf{4 3 5}$

Schmahl, G.P. (1990) Community structure and ecology of sponges associated with four southern Florida coral reefs. New Perspectives in Sponge Biology (ed. by K. Rützler), pp. 376-383. Smithsonian Institution Press, Washington, D.C.

Semmens, B.X., Auster, P.J. \& Paddack, M.J. (2010) Using ecological null models to assess the potential for marine protected area networks to protect biodiversity. PLOS ONE, 5, e8895

Shanks, A.L. \& Shearman, R.K. (2009) Paradigm lost? Cross-shelf distributions of intertidal invertebrate larvae are unaffected by upwelling or downwelling. Marine Ecology Progress Series, 385, 189-204

Shears, N.T. \& Babcock, R.C. (2007) Quantitative description of mainland New Zealand's shallow subtidal reef communities. Science for Conservation, 1-126

Shears, N.T., Smith, F., Babcock, R.C., Duffy, C.A.J. \& Villouta, E. (2008) Evaluation of biogeographic classification schemes for conservation planning: application to New Zealand's coastal marine environment. Conservation Biology, 22, 247-481

Sheppard, C.J.R. (1995) The Shifting Baseline Syndrome. Marine Pollution Bulletin, 30, 766-767

Sloan, N. \& Bartier, P. (2009) Historic marine invertebrate species inventory: case study of a science baseline towards establishing a marine conservation area. Aquatic Conservation: Marine and Freshwater Ecosystems, 19, 827-837

Smith, F.G.W. (1941) Sponge disease in British Honduras, and its transmission by water currents. Ecology, 22, 415-421

Smith, P.J., L., M.J. \& Machin, P.J. (1980) Genetic variation in the rock lobsters Jasus edwardsii and Jasus novaehollandiae. New Zealand Journal of Marine and Freshwater Research, 14, 55-63

Snelder, T., Leathwick, J., Dey, K., Rowden, A., Weatherhead, M., Fenwick, G., Francis, M., Gorman, R., Grieve, J., Hadfield, M., Hewitt, J., Richardson, K., Uddstrom, M. \& Zeldis, J. (2007) Development of an ecologic marine classification in the New Zealand region. Environmental Management, 39, 12-29

Solé-Cava, A.M. \& Boury-Esnault, N. (1999) Patterns of intra and interspecific divergence in marine sponges. Memoirs of the Queensland Museum, 44, 591-602

Solé-Cava, A.M., Boury-Esnault, N., Vacelet, J. \& Thorpe, J.P. (1992) Biochemical genetic divergence and systematics in sponges of the genera Corticium and Oscarella (Demospongiae: Homoscleromorpha) in the Mediterranean Sea. Marine Biology, 113, 299-304

Solé-Cava, A.M., Klautau, M., Boury-Esnault, N., Borojevic, R. \& Thorpe, J.P. (1991) Genetic evidence for cryptic speciation in allopatric populations of two cosmopolitan species of the calcareous sponge genus Clathrina. Marine Biology, 111, 381-386

Sollas, I.B.J. (1902) On the sponges collected during the Skeat expedition in the Malay Peninsula (18991900). Proceedings of the Zoological Society of London, 2, 210-221

Steinitz, O., Heller, J., Tsoar, A., Rotem, D. \& Kadmon, R. (2005) Predicting regional patterns of similarity in species composition for conservation planning. Conservation Biology, 19, 1978-1988

Stendera, S.E.S. \& Johnson, R.K. (2005) Additive partitioning of aquatic invertebrate species diversity across multiple spatial scales. Freshwater Biology, 50, 1360-1375

Stone, A.R. (1970) Growth and reproduction of Hymeniacidon perleve (Montagu) (Porifera) in Langstone Harbour, Hampshire. Journal of Zoology, London., 161, 443-459 
References

Suggate, R.P. (1990) Late pliocene and quaternary glaciations of New Zealand. Quaternary Science Reviews, 9, 175-197

Summerville, K.S. \& Crist, T.O. (2005) Temporal patterns of species accumulation in a survey of Lepidoptera in a beech-maple forest. Biodiversity and Conservation, 14, 3393-3406

Summerville, K.S., Boulware, M.J., Veech, J.A. \& Crist, T.O. (2003) Spatial Variation in Species Diversity and Composition of Forest Lepidoptera in Eastern Deciduous Forests of North America. Conservation Biology, 17, 1045-1057

Tanaka, K. (2002) Growth dynamics and mortality of the intertidal encrusting sponge Halichondria okadai (Demospongiae, Halichondrida). Marine Biology, 140, 383-389

Teder, T., Moora, M., Roosaluste, E., Zobel, K., Pärtel, M., Kõljalg, U. \& Zobel, M. (2007) Monitoring of Biological Diversity: a Common-Ground Approach. Monitoreo de la Diversidad Biológica: un Método con Denominador Común. Conservation Biology, 21, 313-317

Terlizzi, A., Anderson, M.J., Bevilacqua, S., Fraschetti, S., Włodarska-Kowalczuk, M. \& Ellingsen, K.E. (2009) Beta diversity and taxonomic sufficiency: Do higher-level taxa reflect heterogeneity in species composition? Diversity and Distributions, 15, 450-458

Terlizzi, A., Bevilacqua, S., Fraschetti, S. \& Boero, F. (2003) Taxonomic sufficiency and the increasing insufficiency of taxonomic expertise. Marine Pollution Bulletin, 46, 556-561

Tilman, D., Reich, P.B. \& Knops, J.M.H. (2006) Biodiversity and ecosystem stability in a decade-long grassland experiment. Nature, 441, 629-632

Tilman, D., Reich, P.B., Knops, J., Wedin, D., Mielke, T. \& Lehman, C. (2001) Diversity and Productivity in a Long-Term Grassland Experiment. Science, 294, 843-845

Tittensor, D.P., Mora, C., Jetz, W., Lotze, H.K., Ricard, D., Berghe, E.V. \& Worm, B. (2010) Global patterns and predictors of marine biodiversity across taxa. Nature, 466, 1098-1101

Tolimieri, N. \& Anderson, M.J. (2010) Taxonomic Distinctness of Demersal Fishes of the California Current: Moving Beyond Simple Measures of Diversity for Marine Ecosystem-Based Management. PLoS ONE, 5, e10653

Turon, X., Tarjuelo, I. \& Uriz, M.J. (1998) Growth dynamics and mortality of the encrusting sponge Crambe crambe (Poecilosclerida) in contrasting habitats: correlation with population structure and investment in defence. Functional Ecology, 12, 631-639

Tuzet, 0. (1973) Eponges calcaires. Spongiaires 7th Traité de Zoologie (ed. by P.P. Grassé), pp. 27-132. Masson \& Cø, Paris.

Ugland, K.I., Gray, J.S. \& Ellingsen, K.E. (2003) The species-accumulation curve and estimation of species richness. Journal of Animal Ecology, 72, 888-897

Underwood, A.J., Kingsford, M.J. \& Andrew, N.L. (1991) Patterns in shallow subtidal marine assemblages along the coast of New South Wales. Austral Ecology, 16, 231-249

Usher, K.M., Sutton, D.C., Toze, S., Kuo, J. \& Fromont, J. (2004) Biogeography and phylogeny of Chondrilla species (Demospongiae) in Australia. Marine Ecology Progress Series, 270, 117-127

Vacelet, J., Gaino, E., Gallissian, M.F. \& Vacelet, E. (1994) Bacterial attack of spongin skeleton during the 1986-1990 Mediterranean sponge disease. Sponge in time and space; Biology, Chemistry, Paleontology (ed. by R.W.M.V. Soest, T.G. Van Kempen and J.C. Braekman), pp. 355-363. A.A. Balkema, Rotterdam.

van de Vyver, G. (1975) Phenomena of cellular recognition in sponges. Current Topics in Developmental Biology, pp. 123-140. Academic Press, New York. 
van Soest, R.W.M. (1978) Marine sponges from Curaçao and other Caribbean localities Part I. Keratosa. Studies on the Fauna of Curaçao and other Caribbean Islands (ed. by P. Wagenaar Hummelinck and L.J. Van Der Steen), pp. 1-94. Foundation for Scientific Research in Surinam and the Netherlands Antilles, Amsterdam.

van Soest, R.W.M. (1980) Marine sponges from Curaçao and other Caribbean localities. Part II. Haplosclerida. Studies on the Fauna of Curaçao and other Caribbean Islands (ed. by P. Wagenaar Hummelinck and L.J. Van Der Steen), pp. 1-173. Foundation for Scientific Research in Surinam and the Netherlands Antilles, Amsterdam.

van Soest, R.W.M. (1984) Marine sponges from Curaçao and other Caribbean localities Part III. Poecilosclerida. Studies on the Fauna of Curaçao and other Caribbean Islands (ed. by P. Wagenaar Hummelinck and L.J. Van Der Steen), pp. 1-167. Foundation for Scientific Research in Surinam and the Netherlands Antilles, Amsterdam.

van Soest, R.W.M. (1989) The Indonesian sponge fauna: a status report. Netherlands Journal of Sea Research, 23, 223-230

van Soest, R.W.M. (1990) Shallow water reef sponges of Eastern Indonesia. New Perspectives in Sponge Biology (ed. by K. Rützler), pp. 302-308. Smithsonian Institution Press, Washington, D.C.

van Soest, R.W.M. (1994) Demosponge distribution patterns. Sponge in time and space; Biology, Chemistry, Paleontology (ed. by R.W.M.V. Soest, T.G. Van Kempen and J.C. Braekman), pp. 213-223. A.A. Balkema, Rotterdam.

van Soest, R.W.M. (2007) Sponge biodiversity. Journal of the Marine Biological Association of the United Kingdom , 87, 1345-1348

van Soest, R.W.M., Boury Esnault, N., Hooper, J.N.A., Rützler, K., de Voogd, N.J., Alvarez de Glasby, B., Hajdu, E., Pisera, A.B., Vacelet, J., Manconi, R., Schoenberg, C., Janussen, D., Tabachnick, K.R. \& Klautau, M. (2008) World Porifera database. Available at: http://www.marinespecies.org/porifera/ (accessed 30-08-2010)

Veech, J.A. \& Crist, T.O. (2010a) Toward a unified view of diversity partitioning. Ecology, 91, 1988-1992

Veech, J.A. \& Crist, T.O. (2010b) Diversity partitioning without statistical independence of alpha and beta. Ecology, 91, 1964-1969

Veech, J.A., Summerville, K.S., Crist, T.O. \& Gering, J.C. (2002) The additive partitioning of species diversity: recent revival of an old idea. Oikos, 99, 3-9

Voultsiadou, E. (2009) Reevaluating sponge diversity and distribution in the Mediterranean Sea. Hydrobiologia, 628, 1-12

Walker, B.H. (1992) Biodiversity and ecological redundancy. Conservation Biology, 6, 18-23

Walls, K. (1995) The New Zealand experience in developing a marine biogeographic regionalisation. In: Towards a marine regionalisation for Australia: proceedings of a workshop. (ed. J. Muldoon), pp. 33-48. Great Barrier Reef Marine Park Authority, Townsville, Australia

Wapstra, M. \& Soest, R.W.M.V. (1987) Sexual reproduction, larval morphology and behaviour in demosponges from the south-west of the Netherlands. Taxonomy of Porifera from the N.E. Atlantic and Mediterranean Sea. NATO-ASI Series (ed. by J. Vacelet and N. Boury-Esnault), pp. 281308. Springer-Verlag, Berlin.

Warwick, R.M. \& Turk, S.M. (2002) Predicting climate change effects on marine biodiversity: comparison of recent and fossil molluscan death assemblages. Journal of the Marine Biological Association of the United Kingdom, 82, 847-850 
References

Waters, J.M. \& Roy, M.S. (2003) Marine biogeography of southern Australia: phylogeographical structure in a temperate sea-star. In, pp. 1787-1796. Blackwell Science Ltd

Webster, N.S. (2007) Sponge disease: a global threat? Environmental Microbiology, 9, 1363-1375

Wheeler, Q.D. (2004) Taxonomic triage and the poverty of phylogeny. Philosophical Transactions of the Royal Society of London Series B-Biological Sciences, 359, 571-583

White, E.P., Ernest, S.K.M., Adler, P.B., Hurlbert, A.H. \& Lyons, S.K. (2010) Integrating spatial and temporal approaches to understanding species richness. Philosophical Transactions of the Royal Society B: Biological Sciences, 365, 3633-3643

Whittaker, R.H. (1960) Vegetation of the Siskiyou Mountains, Oregon and California. Ecological Monographs, 30, 279-338

Whittaker, R.H. (1972) Evolution and Measurement of Species Diversity. Taxon, 21, 213-251

Wilkinson, C.R. (1987) Interocean differences in size and nutrition of coral reef sponge populations. Science, 236, 1654-1657

Wilkinson, C.R. \& Cheshire, A.C. (1988) Growth rate of Jamaican coral reef sponges after Hurricane Allan. Biological Bulletin, 175, 175-179

Wilkinson, C.R. \& Cheshire, A.C. (1989) Patterns in the distribution of sponge populations across the Central Great Barrier Reef. Coral Reefs, 8, 127-134

Wilkinson, C.R. \& Cheshire, A.C. (1990) Comparisons of sponge populations across the barrier reefs of Australia and Belize: Evidence for higher productivity in the Caribbean. Marine Ecology Progress Series, 67, 285-294

Wilkinson, C.R. \& Evans, E. (1989) Sponge distribution across Davies Reef, Great Barrier Reef, relative to location, depth, and water movement. Coral Reefs, 8, 1-7

Wilkinson, C.R. \& Trott, L.A. (1983) Biogeographic distribution of sponges on an East-West transect across the Great Barrier Reef. Bulletin of Marine Science, 33, 784

Wilkinson, C.R. \& Vacelet, J. (1979) Transplantation of marine sponges to different conditions of light and current. Journal of Experimental Marine Biology and Ecology, 37, 91-104

Willis, K.J. \& Whittaker, R.J. (2002) Species Diversity--Scale Matters. Science, 295, 1245-1248.

Willis, K.J., Araújo, M.B., Bennett, K., D., Figueroa-Rangel, B., Froyd, C.A. \& Myers, N. (2007) How can a knowledge of the past help to conserve the future? Biodiversity conservation and the relevance of long-term ecological studies. Philosophical Transactions of the Royal Society B: Biological Sciences, 362, 175-186

Wilson, E.0. (2004) Taxonomy as a fundamental discipline. Philosophical Transactions: Biological Sciences, 359, 739

Witman, J.D., Etter, R.J. \& Smith, F. (2004) The relationship between regional and local species diversity in marine benthic communities: A global perspective. Proceedings of the National Academy of Sciences of the United States of America, 101, 15664-15669

Wood, S.N. (2006a) Generalized additive models: an introduction with R. Chapman and Hall/CRC.

Wood, S.N. (2006b) Low-rank scale-invariant tensor product smooths for generalized additive mixed models. Biometrics, 62, 1025-1036

Wörheide, G. \& Erpenbeck, D. (2007) DNA taxonomy of sponges - progress and perspectives. Journal of the Marine Biological Association of the United Kingdom , 87, 1629-1633

Wörheide, G., Epp, L.S. \& Macis, L. (2008) Deep genetic divergences among Indo-Pacific populations of the coral reef sponge Leucetta chagosensis (Leucettidae): Founder effects, vicariance, or both? Bmc Evolutionary Biology, 8, 24 
References

Wörheide, G., Solé-Cava, A.M. \& Hooper, J.N.A. (2005) Biodiversity, molecular ecology and phylogeography of marine sponges: Patterns, implications and outlooks. Integrative and Comparative Biology, 45, 377-385

Worm, B., Barbier, E.B., Beaumont, N., Duffy, J.E., Folke, C., Halpern, B.S., Jackson, J.B.C., Lotze, H.K., Micheli, F., Palumbi, S.R., Sala, E., Selkoe, K.A., Stachowicz, J.J. \& Watson, R. (2006) Impacts of biodiversity loss on ocean ecosystem services. Science, 314, 787 - 790

Worm, B., Sandow, M., Oschlies, A., Lotze, H.K. \& Myers, R.A. (2005) Global patterns of predator diversity in the open oceans. Science, 309, 1365-1369

Wu, F., Yang, X.J. \& Yang, J.X. (2010) Additive diversity partitioning as a guide to regional montane reserve design in Asia: an example from Yunnan Province, China. Diversity and Distributions, 16, 10221033

Wulff, J.L. (1991) Asexual fragmentation, genotype success, and population dynamics of erect branching sponges. Journal of Experimental Marine Biology and Ecology, 149, 227-247

Wulff, J.L. (1994) Sponge feeding by Caribbean angelfishes, trunkfishes, and filefishes. Sponge in time and space; Biology, Chemistry, Paleontology (ed. by R.W.M.V. Soest, T.G. Van Kempen and J.C. Braekman), pp. 265-271. A.A. Balkema, Rotterdam.

Wulff, J.L. (1995a) Effects of a hurricane on survival and orientation of large erect coral reef sponges. Coral Reefs, 14, 55

Wulff, J.L. (1995b) Sponge-feeding by the Caribbean starfish Oreaster reticulatus. Marine Biology, 123, 313325

Wulff, J.L. (1996) Do the same sponge species live on both the Caribbean and eastern Pacific sides of the Isthmus of Panama. Bulletin del'Institut royal des Sciences naturelles de Belgique, Biologie, 66, 165-173

Wulff, J.L. (1997a) Mutualisms among species of coral reef sponges. Ecology, 78, 146-159

Wulff, J.L. (1997b) Parrotfish predation on cryptic sponges of Caribbean coral reefs. Marine Biology, 129, 41-52

Wulff, J.L. (2000) Sponge predators may determine differences in sponge fauna between two sets of mangrove cays, Belize Barrier Reef. Atoll Research Bulletin, 251-263

Wulff, J.L. (2001) Assessing and monitoring coral reef sponges: Why and how? Bulletin of Marine Science, 69, 831-846

Wulff, J.L. (2006a) Ecological interactions of marine sponges. Canadian Journal of Zoology, 84, 146-166

Wulff, J.L. (2006b) Rapid diversity and abundance decline in a Caribbean coral reef sponge community. Biological Conservation, 127, 167-176

Wulff, J.L. (2006c) Resistance vs recovery: morphological strategies of coral reef sponges. Functional Ecology, 20, 699-708

Wulff, J.L. (2007) Disease prevalence and population density over time in three common Caribbean coral reef sponge species. Journal of the Marine Biological Association of the United Kingdom, 87, 17151720

Wulff, J.L. (2008) Collaboration among sponge species increases sponge diversity and abundance in a seagrass meadow. Marine Ecology, 29, 193-204

Wulff, J.L. \& Buss, L.W. (1979) Do sponges help hold coral reefs together? Nature, 281, 474-475

Xavier, J. (2009) Biodiversity and phylogeography of Northeast Atlantic and Mediterranean sponges. University of Amsterdam, Amsterdam. 
References

Yachi, S. \& Loreau, M. (2006) Biodiversity and ecosystem productivity in a fluctuating environment: The insurance hypothesis. Proceedings of the National Academy of Sciences of the United States of America. , 96, 1463-1468

Yachi, S. \& Loreau, M. (2006) Biodiversity and ecosystem productivity in a fluctuating environment: The insurance hypothesis. Proceedings of the National Academy of Sciences of the United States of America. , 96, 1463-1468

Zea, S. (1993) Recruitment of demosponges (Porifera, Demospongiae) in rocky and coral reef habitats of Santa Marta, Colombian Caribbean. Marine Ecology, 14, 1-21

Zea, S. (1994) Patterns of coral and sponge abundance in stressed coral reefs at Santa Marta, Colombian Caribbean. Sponge in time and space; Biology, Chemistry, Paleontology (ed. by R.W.M.V. Soest, T.G. Van Kempen and J.C. Braekman), pp. 257-264. A.A. Balkema, Rotterdam.

Zvuloni, A., van Woesik, R. \& Loya, Y. (2010) Diversity partitioning of stony corals across multiple spatial scales around Zanzibar Island, Tanzania. PLoS ONE, 5, e9941 